\title{
FLOW THROUGH A SOLAR CHIMNEY POWER PLANT COLLECTOR-TO-CHIMNEY TRANSITION SECTION
}

\author{
CARL KIRSTEIN
}

Thesis presented in partial fulfilment of the requirements for the degree of Master of engineering science at the University of Stellenbosch

December 2004

Under Supervision of:

Prof T.W. Von Backstrom

Prof D.G. Kroger 
I, the undersigned, hereby declare that the work presented in this thesis is my own original work and that I have not previously in its entirety or in part submitted it at any university for a degree

Signed:

Date: 


\section{SUMMARY}

One of the areas of the fluid dynamic design of solar chimney power plants that has not been investigated sufficiently is the collector-to-chimney transition section of a single turbine layout. The transition section contains the turbine inlet guide vanes (IGVs) that support the whole chimney and guide the flow entering the turbine. The primary objective of the study was to determine the dependence of the loss coefficient of the section on inlet guide vane stagger angle and collector roof height. Experiments were done on a nominal $900 \mathrm{~mm}$ chimney diameter rig, with four combinations of two collector roof heights and two IGV stagger angles. Velocity components and pressures in the transition section were measured in three conical planes, respectively at the IGV exit and midway to, and just below the turbine position, using a five-hole pneumatic pressure probe. Very good agreement was found between experimental values and commercial CFD code predictions of flow angles, velocity components and internal and wall static pressures. The agreement between measured and predicted total pressure loss coefficient was reasonable when considering that most of the loss occurred in the weak wakes of the IGVs and in the very thin transition section wall boundary layers. The CFD code served to extend the predictions to a proposed full scale geometry. The losses are less than previously assumed. The study led to correlations between respectively loss and turning angle as dependent variables, and collector roof height and IGV stagger angle as independent variables. 


\section{OPSOMMING}

Een van die gebiede van vloeidinamiese ontwerp van sonskoorsteen kragstasies wat nog nie voldoende navorsing geniet het nie is die kollektor-tot-skoorsteen oorgangs gedeelte van 'n enkel turbine opstelling. Die oorgangs gedeelte bevat die turbine se inlaat lei lemme (ILL) wat die hele skoorsteen dra en die vloei lei wat by die turbine ingaan. Die hoof doel van hierdie studie was om die verlies koëffisiënt van hierdie oorgangs gedeelte te bepaal as afhanklike van die ILL stel hoek en die hoogte van die kollektor se dak hoogte. Die eksperimente het op 'n nominale $900 \mathrm{~mm}$ deursnee skoorsteen skaal model geskied, met vier kombinasies van twee dak hoogtes en twee ILL stel hoeke. Snelheidskomponente en drukke is met 'n pneumatiese 5-punt buis in drie koniese vlakke in die oorgangs gedeelte gemeet. Die vlakke was by die ILL se stert, halfpad deur die oorgangs gedeelte en by die turbine se inlaat. Baie goeie ooreenstemming is gevind met die eksperimentele waardes en 'n kommersiële CFD kode se voorspellings van vloei hoeke, snelheidskomponente en interne- en wand statiese drukke. Die ooreenstemming tussen die gemete waardes en die berekende waardes vir die totale druk verlies koëffisiënt was redelik siende dat die meeste verliese van die klein versteurings van die ILL en die oorgangs gedeelte se dun-wand grenslae kom. Die CFD kode is toe ingespan om verdere voorspellings te maak vir 'n voorgestelde volskaal geometrie. Die verliese is minder as wat daar van te vore voorspel is. Hierdie studie het gelei tot korrelasies tussen onderskeidelik verlies en draai hoeke as afhanklike veranderlikes, en kollektor dak hoogte en ILL plasings hoek as onafhanklike veranderlikes. 


\section{ACKNOWLEDGEMENTS}

This thesis I dedicate wholly to the One who sought me until He found me, even though I did not seek Him nor realised how much I needed Him. To you my Lord Jesus Christ I dedicate this entire project. I may be getting another degree, but during this master's degree I found many more riches and treasures than I bargained for. Thank you for making my life one big blissful celebration.

Professor Kröger remarked that if you finish a master's degree, the rest of your life is easy. I do not know whether this is true, but this master's degree has certainly been challenging enough that I suspect he may be right. I have been crushed as grapes to produce wine and have grown in character and professionalism henceforth. However the crushing was not easy to bear, but thankfully I have had such incredible friends and family who were there to support me and push me in the times I needed it.

Thank you professor Von Backström for inspiring me to attain excellence and professor Kröger for your sponsorship. Thank you to the effervescent Nicolà Cencelli, at last a very dear friend; Cobus van Dyk the inspirational visionary and comrade pilgrim; Cobus and Ferdi Zietsman and Moses Siebrits that never complain or hesitate to help out and give excellent advice; Annie, Jessica and Johnny on the $6^{\text {th }}$ floor with radiant faces and excellent company; my cell group that was always there with support, advice, guidance and food for the body, soul and spirit; Jaco my dear brother, humble, excellent and gentle, always on the same ridiculous frequency of thought as me, what a pleasure to have you as brother and as a friend. And finally to my parents and Marno, my little brother, thank you for your tenacity in prayer, support and reaching out to me throughout this thesis. I would certainly have failed had it not been for all of you. 
SUMMARY I

OPSOMMING II III

TABLE OF CONTENTS IV

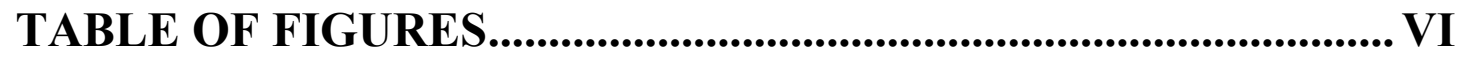

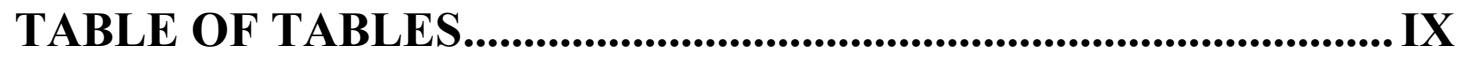

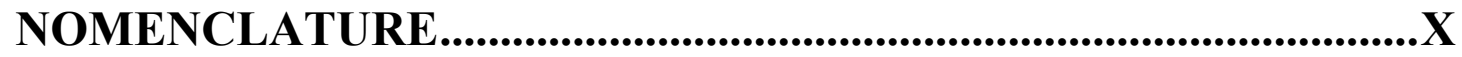

CHAPTER 1： INTRODUCTION ..................................................1

1.1 The Solar ChIMney Power Plant ........................................................... 1

1.2 ApPliCABLE Design Methodologies AND ANALYSIS TOOLS ......................6

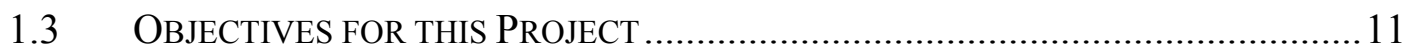

\section{CHAPTER 2: EXPERIMENTAL FLUID DYNAMICS..................13}

2.1 INTRODUCTION TO EXPERIMENTAL MODELLING OF THE SCPP ................... 13

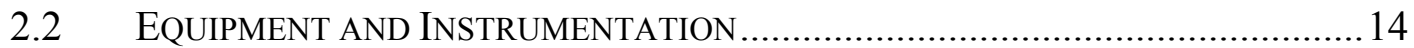

2.2.1 THE SCPP EXPERIMENTAL RIG ....................................................... 14

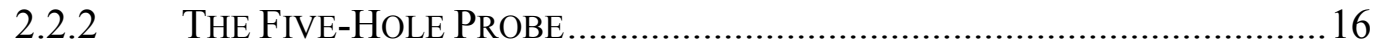

2.2.3 Hottinger BALDWIN MessteChNIK PRESSURE TRANSDUCERS ......... 18

2.2.4 HBM BRIDGE AMPLIFIER ……......................................................... 19

2.2.5 AUTO TRAN ${ }^{\mathrm{TM}}$ 750D-250 PRESSURE TRANSDUCERS ........................... 19

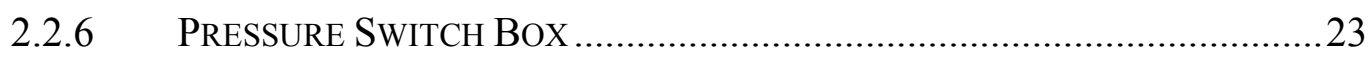

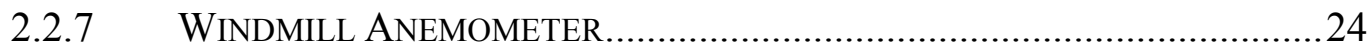

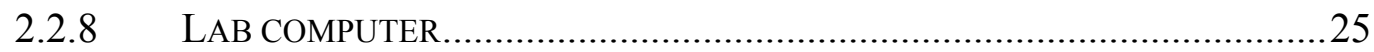

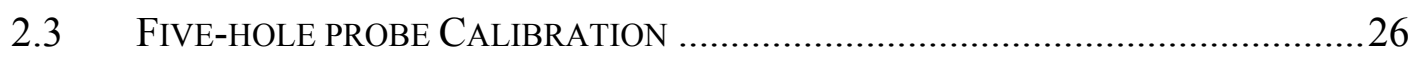

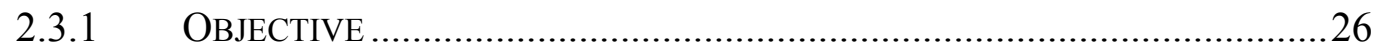

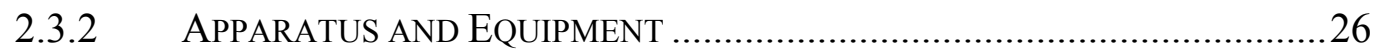

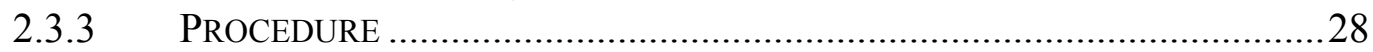

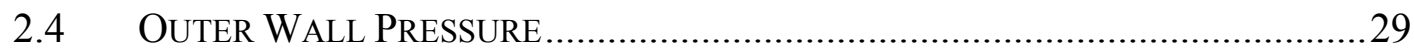

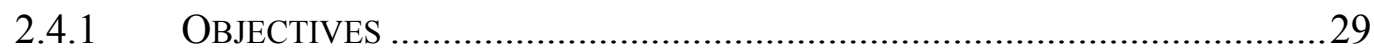

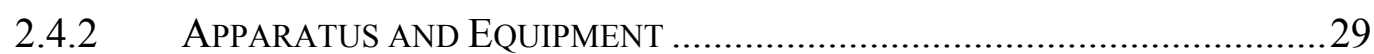

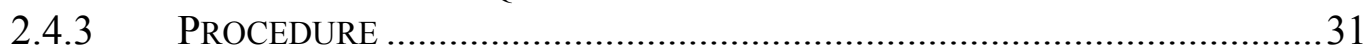

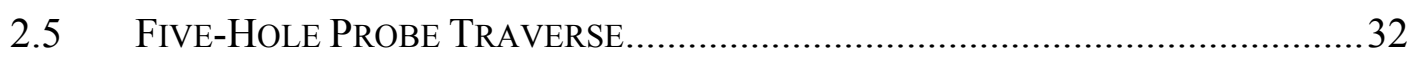

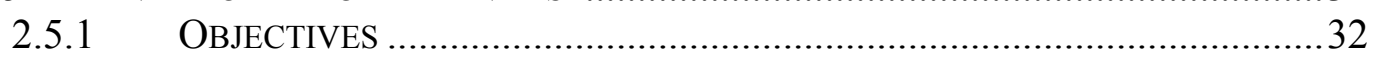

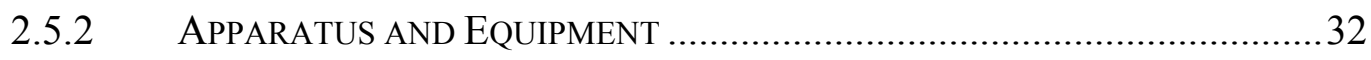

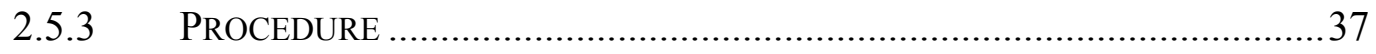




\section{CHAPTER 3: COMPUTATIONAL FLUID DYNAMICS................39}

3.1 InTRODUCTION TO NuMERICAL MODELling OF THE SCPP ..........................39

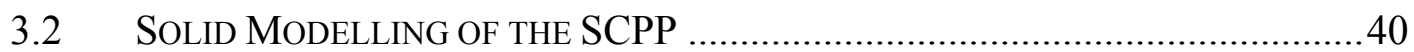

3.2.1 BUILDING THE SOLID MODEL WITH CAD …..........................................4 40

3.2.2 IMPORTING THE SOLID MODEL TO CFX ............................................. 42

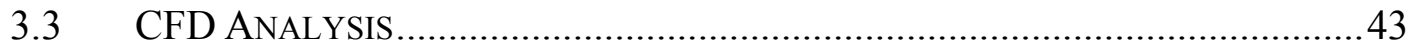

3.3.1 PRELIMINARY INVESTIGATION OF FLOW ............................................ 43

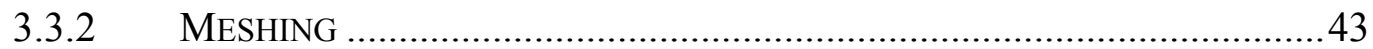

3.3.3 BOUNDARY- AND STARTING CONDITIONS .......................................... 51

3.3.4 ERRORS DUE TO ADVECTION SCHEMES................................................55

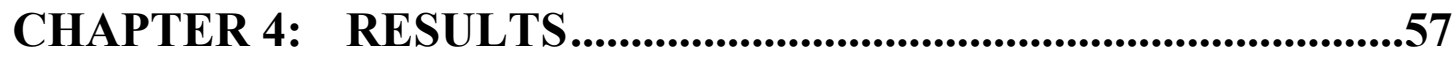

4.1 INTRODUCTION TO THE SYNTHESIS OF THE RESULTS …................................5

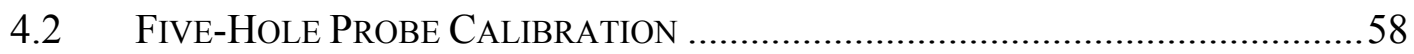

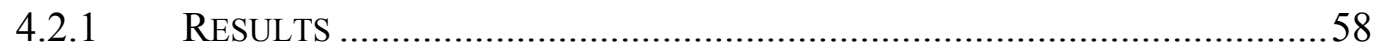

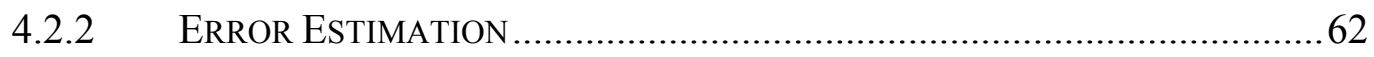

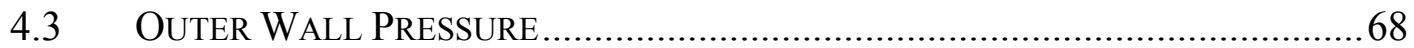

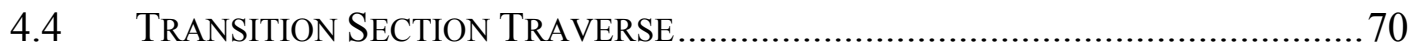

4.4.1 VELOCITY DISTRIBUTIONS AND CONTINUITY .................................... 70

4.4.2 PRESSURE DISTRIBUTIONS AND LOSSES.............................................. 74

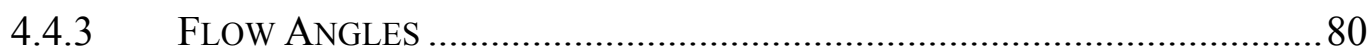

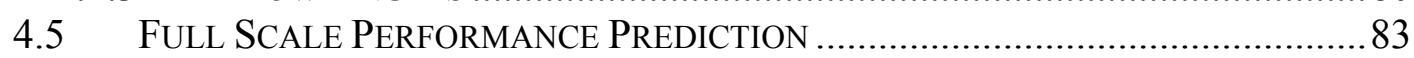

\section{CHAPTER 5: CONCLUSIONS AND RECOMMENDATIONS....89}

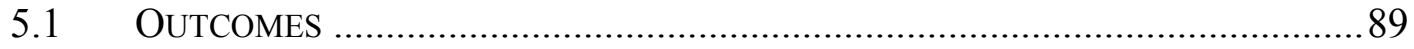

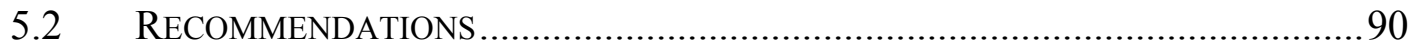

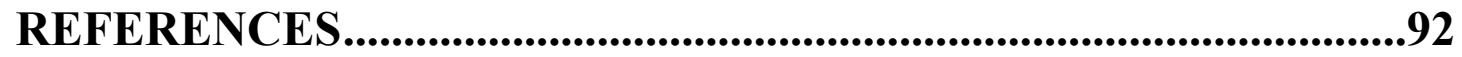

\section{APPENDIX A: CALCULATIONS...........................................................1}

A.1 BOUNDARY LAYER ANALYSIS OF THE OUTER WALL ...................................... 1

A.2 InVESTIGATION OF THE IGV BLADE ROW TORQUE ...................................... 9

A.3 LOAD-TO-STIFFNESS RATIO OF THE IGV CASCADE .................................... 11

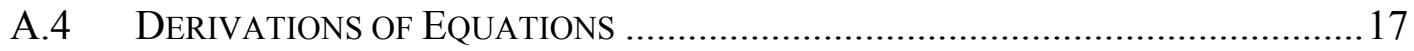

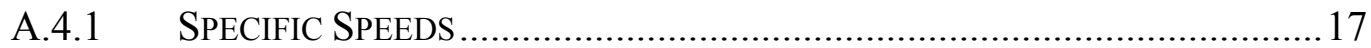

A.4.2 StATIC AND TOTAL PRESSURES ……................................................. 17

A.4.3 ERROR ESTIMATION STATISTICS ....................................................... 18

\section{APPENDIX B： FIGURES OF RESULTS..................................22}

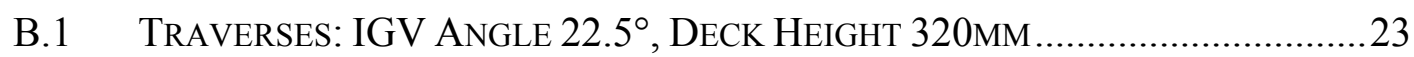

B.2 Traverses: IGV ANGLE $31.5^{\circ}$, DeCK Height 320Mm .................................. 31

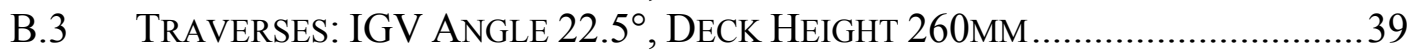

B.4 TRAVERSES: IGV ANGLE $31.5^{\circ}$, DECK HEIGHT 260MM .................................47

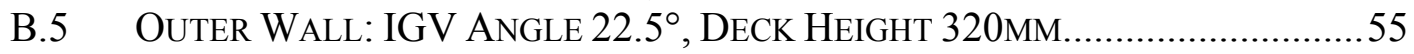

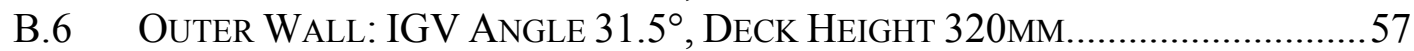

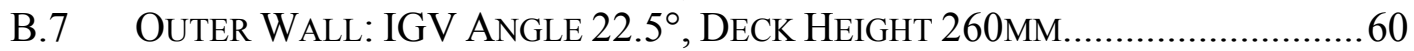

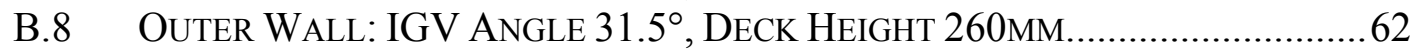




\section{TABLE OF FigURES}

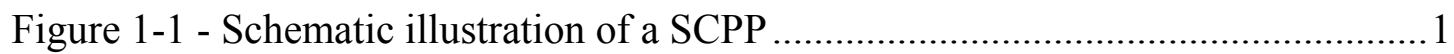

Figure 1-2 - Detail of the SCPP layout near its centre ................................................2

Figure 1-3 - Schematic of the heat transfer in the collector of a SCPP .........................

Figure 1-4 - Full scale transition section relative to a Boeing 747 ............................. 4

Figure 1-5 - Representation of the pilot plant at Manzanares …................................5

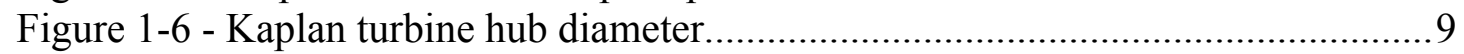

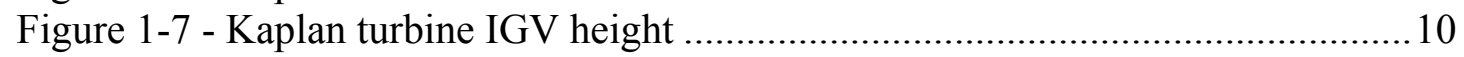

Figure 1-8 - Kaplan turbine IGV position diameter................................................ 10

Figure 2-1 - Performance prediction and calibration by CFD and EFD ..................... 13

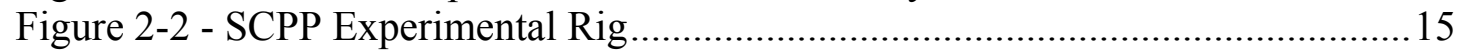

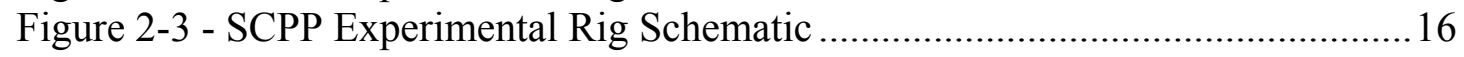

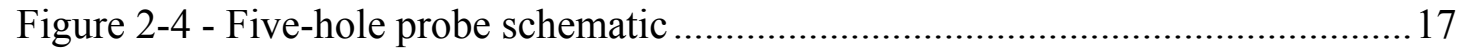

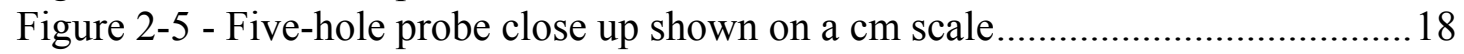

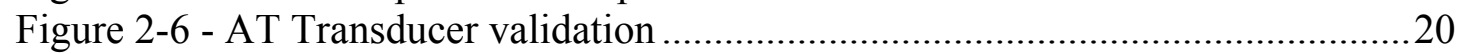

Figure 2-7 - AT Transducer validation zoomed ....................................................21

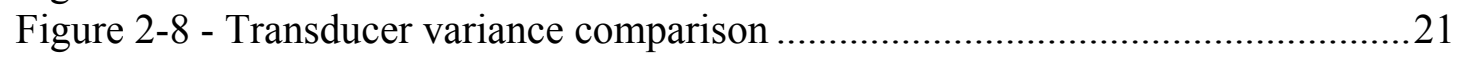

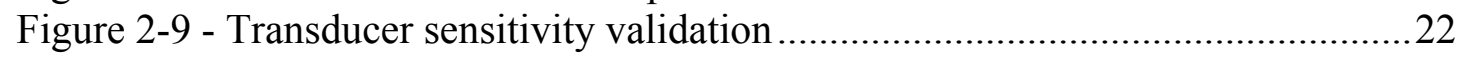

Figure 2-10 - AT Transducers........................................................................... 23

Figure 2-11 - Master and Slave Switch Boxes .......................................................24

Figure 2-12 - Windmill anemometer flow rate $\left[\mathrm{m}^{3} / \mathrm{s}\right]$ readings $\left(22.5^{\circ} 320 \mathrm{~mm}\right) \ldots \ldots \ldots . .25$

Figure 2-13 - Five-hole probe calibration mounting detail......................................27

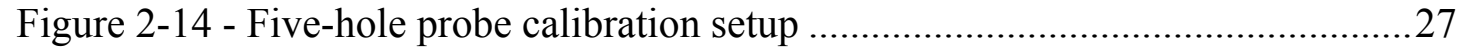

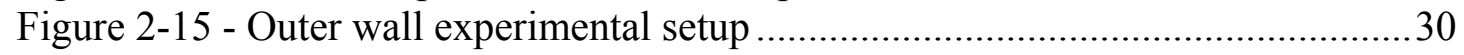

Figure 2-16 - Row location of static pressure tapping points ...................................31

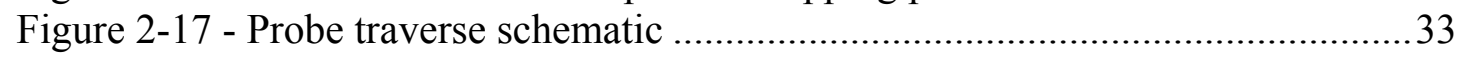

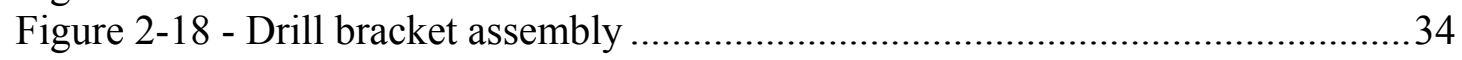

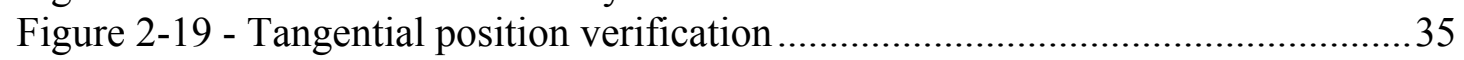

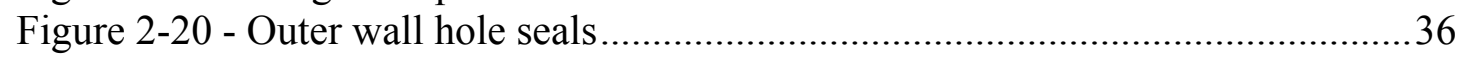

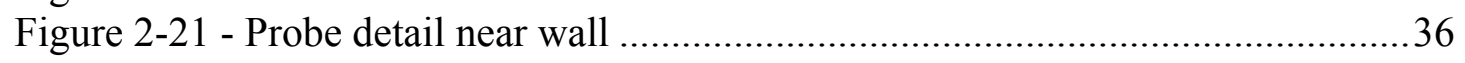

Figure 2-22 - Traverse line positions for the five-hole probe ................................... 37

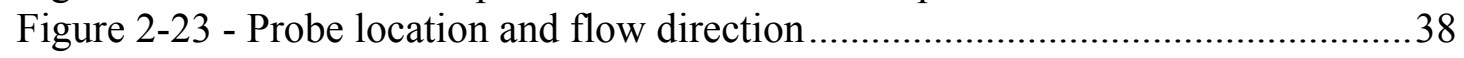

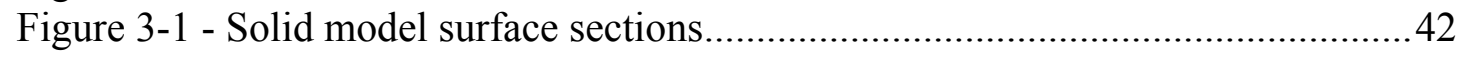

Figure 3-2 - Surface mesh for a 320mm deck height.................................................46

Figure 3-3 - Mesh verification on outer wall; pressure (left axis) and $\mathrm{y}^{+}$(right axis) 47

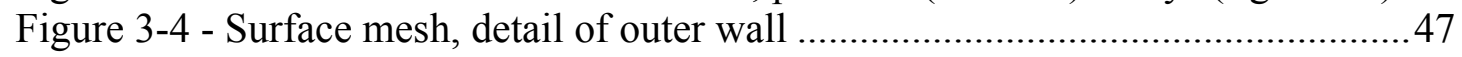

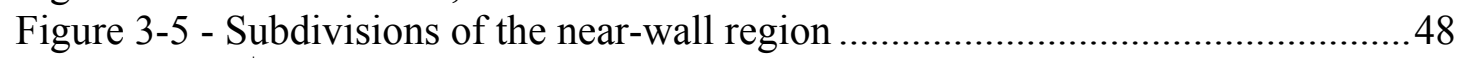

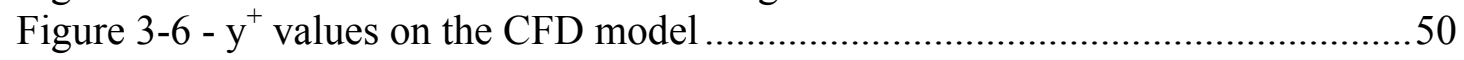

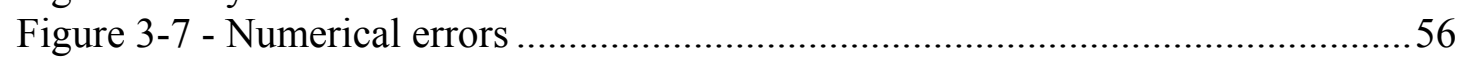

Figure 4-1 - $\mathrm{Cp}_{\text {centre }}(\mathrm{left})$ and $\mathrm{Cp}_{\text {average }}$ (right) Calibration Maps ...............................58

Figure 4-2 - $\mathrm{Cp}_{\text {pitch }}$ (left) and $\mathrm{Cp}$ yaw $($ right) Calibration Maps.....................................59

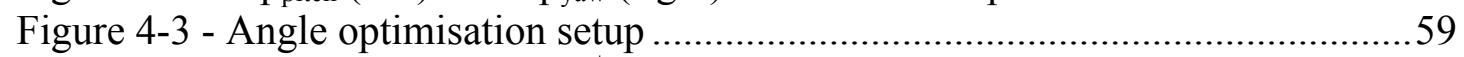

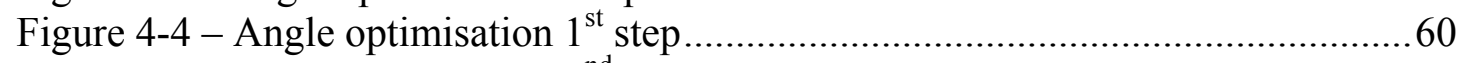

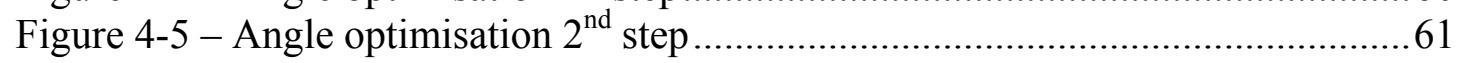

Figure 4-6 - Variance of measurements in the five-hole probe .................................65

Figure 4-7 - Outer wall pressure; IGV angle $22.5^{\circ}$ and deck height $260 \mathrm{~mm}$..............68 


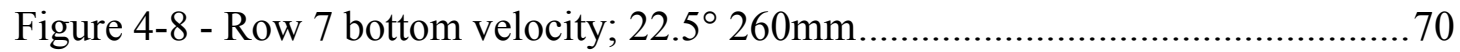

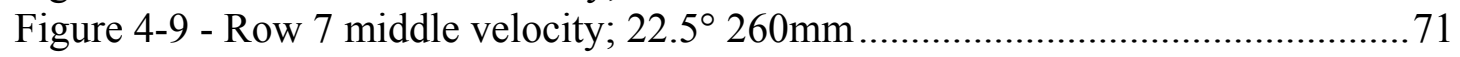

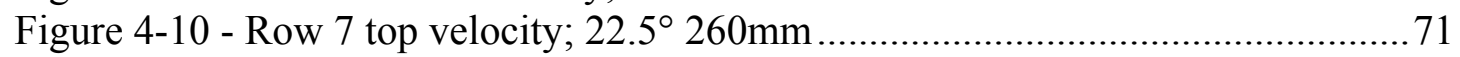

Figure 4-11 - Azimuthal plane integration schematic............................................. 73

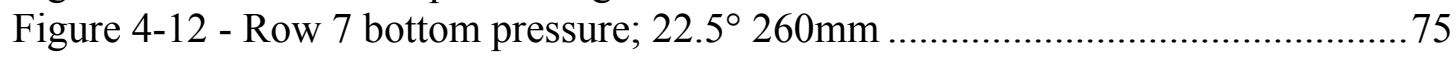

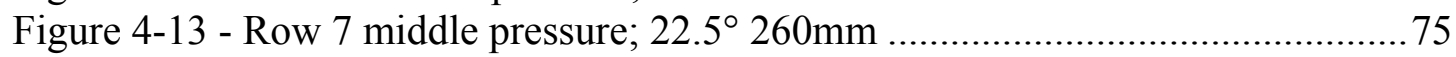

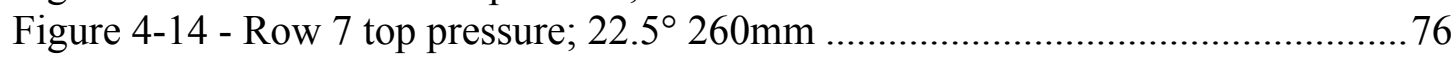

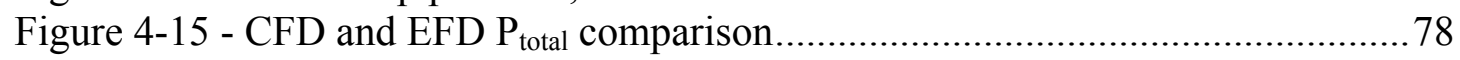

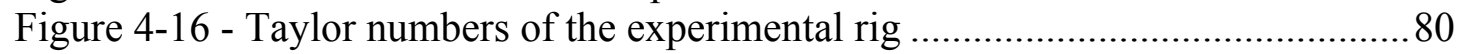

Figure 4-17 - Row 7 bottom angles; 22.5 260mm ................................................ 81

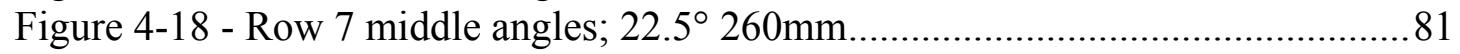

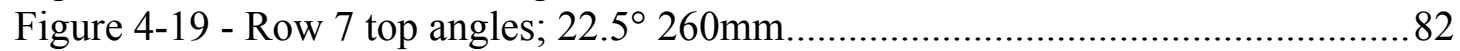

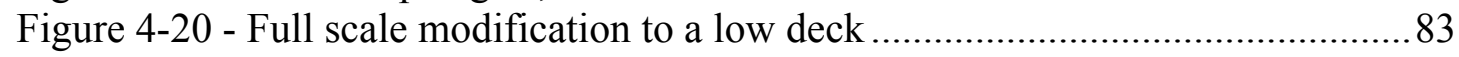

Figure 4-21 - Full scale modification to a high deck .............................................. 84

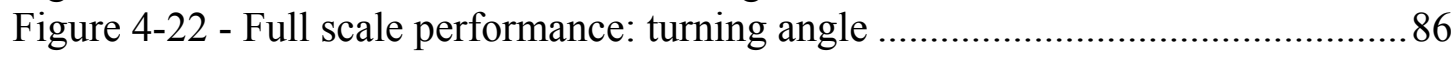

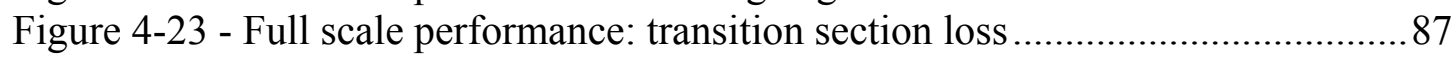

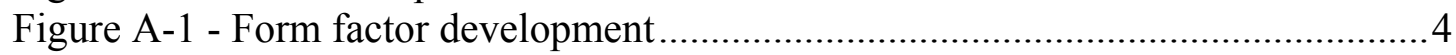

Figure A-2 - Thwaites parameter's development ..................................................

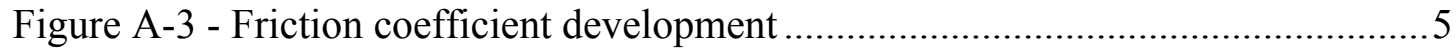

Figure A-4 - Michel's method for transition prediction ............................................ 6

Figure A-5 - Wazzan's method for transition prediction .......................................... 7

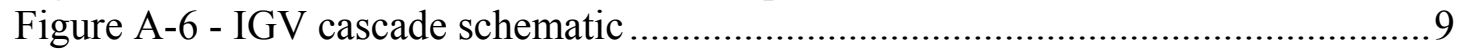

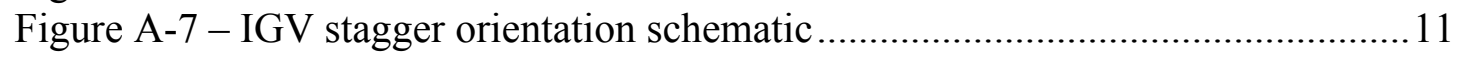

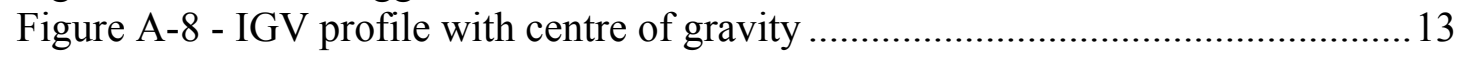

Figure A-9 - Load-to-stiffness ratio of the IGV cascade ......................................... 16

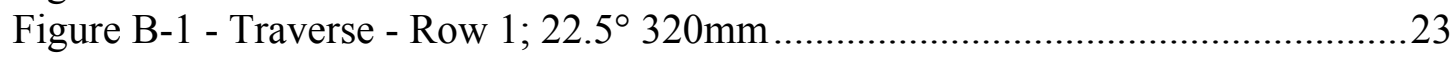

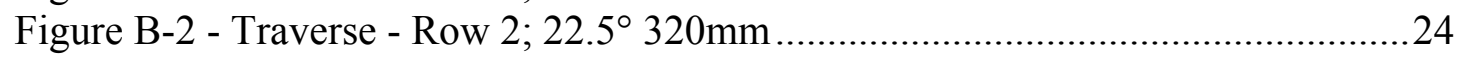

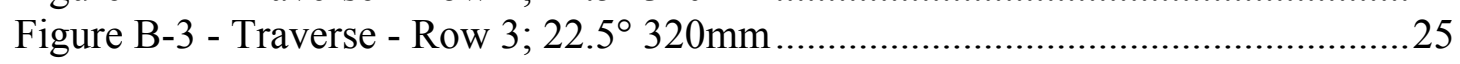

Figure B-4 - Traverse - Row 4 below bottom; 22.5 320mm ..................................26

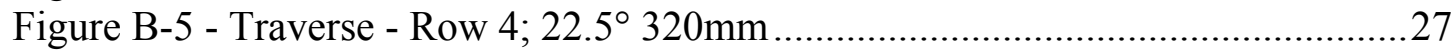

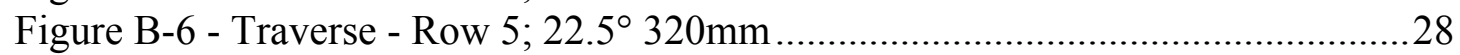

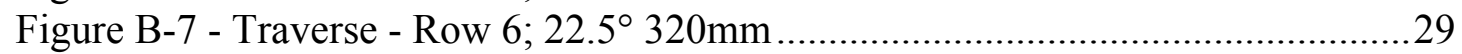

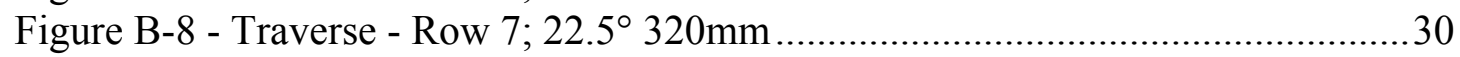

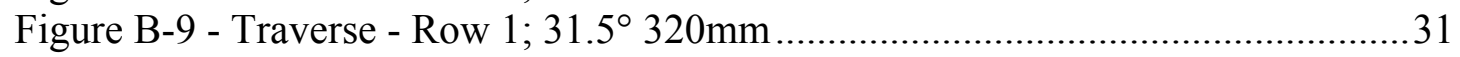

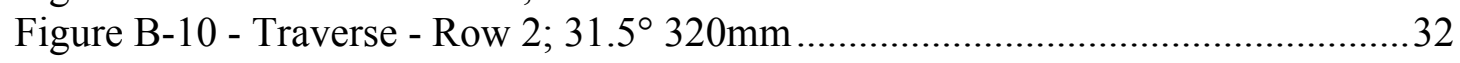

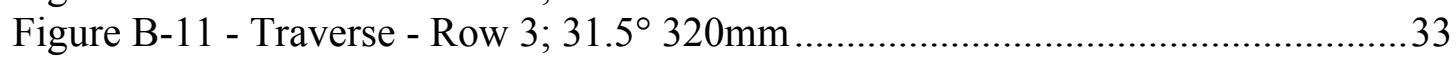

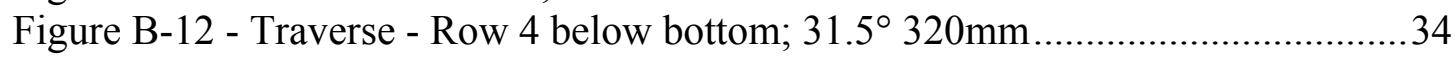

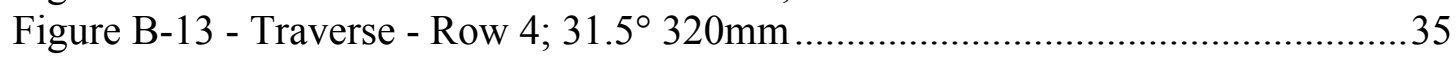

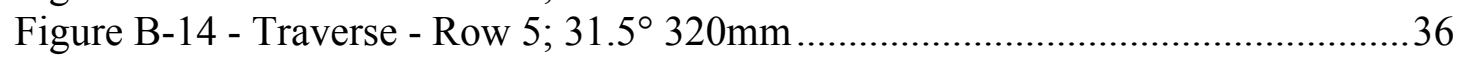

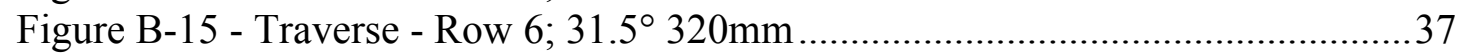

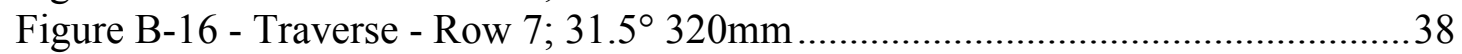

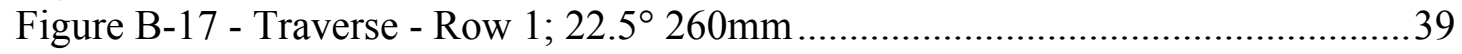

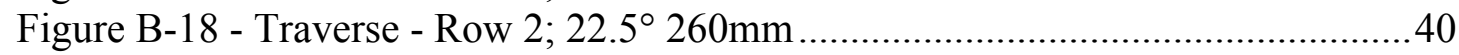

Figure B-19 - Traverse - Row 3; $22.5^{\circ} 260 \mathrm{~mm}$..........................................................4 41

Figure B-20 - Traverse - Row 4 below bottom; 22.5 $260 \mathrm{~mm}$....................................4 42

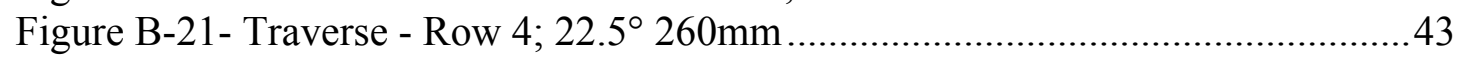

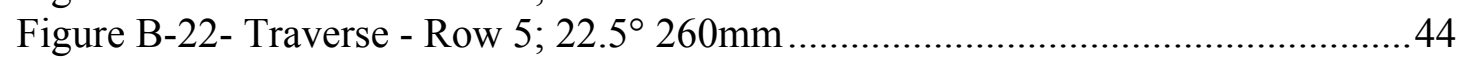

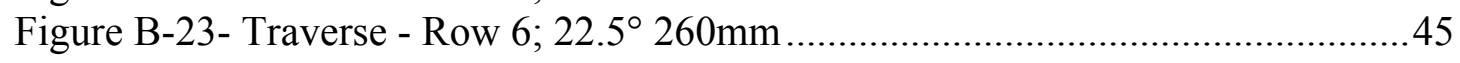

Figure B-24- Traverse - Row 7; $22.5^{\circ} 260 \mathrm{~mm}$............................................................46

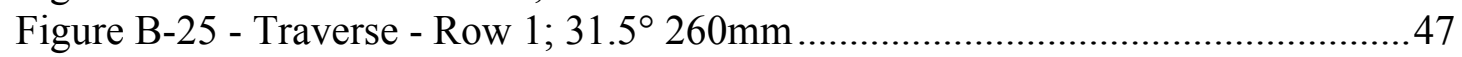




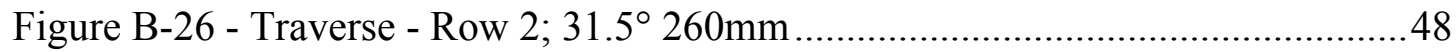

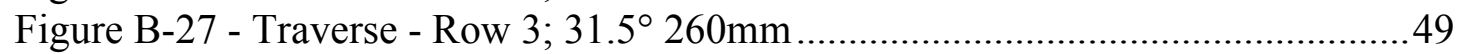

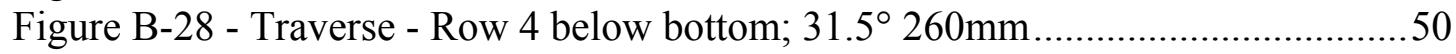

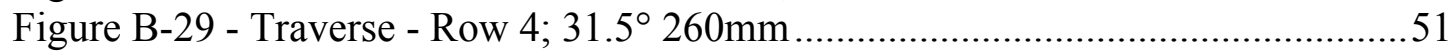

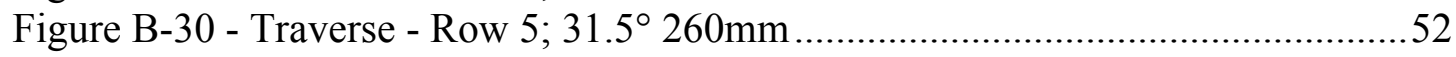

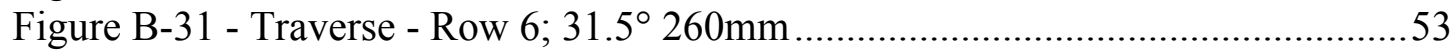

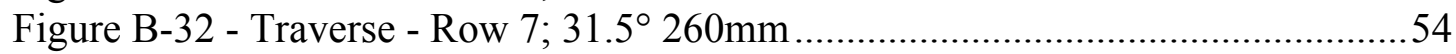

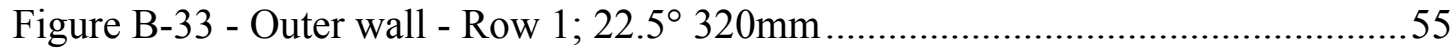

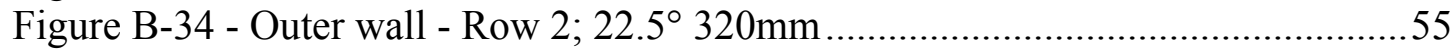

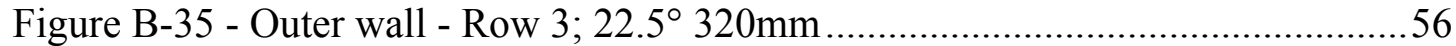

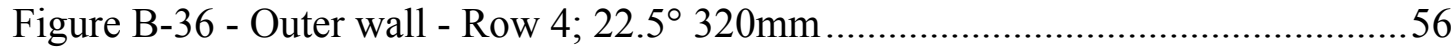

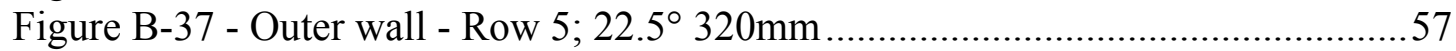

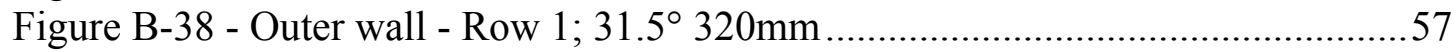

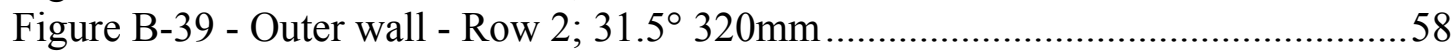

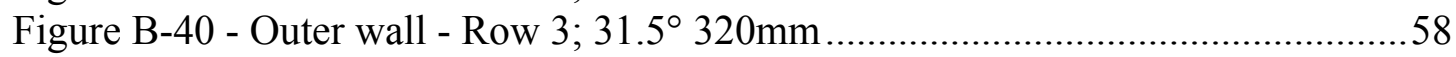

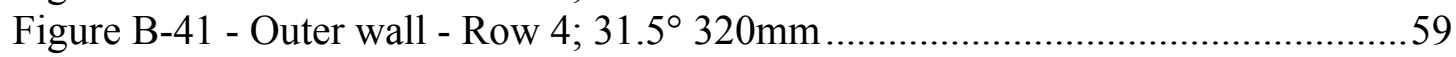

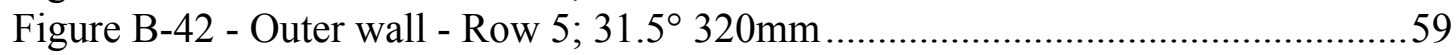

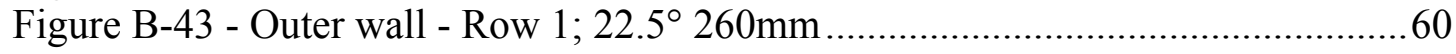

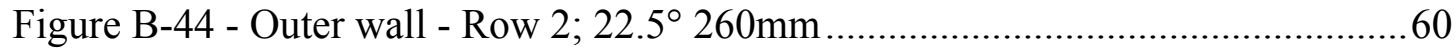

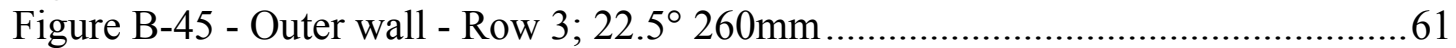

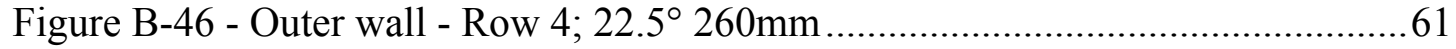

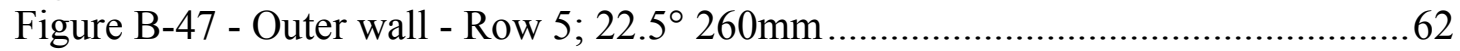

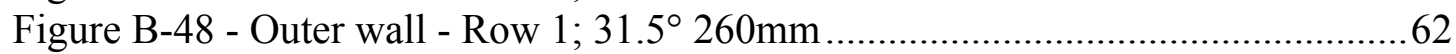

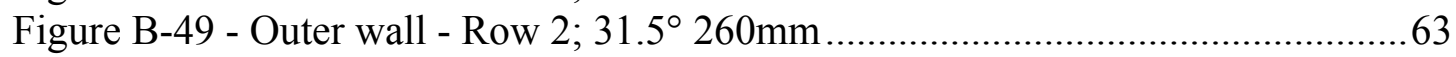

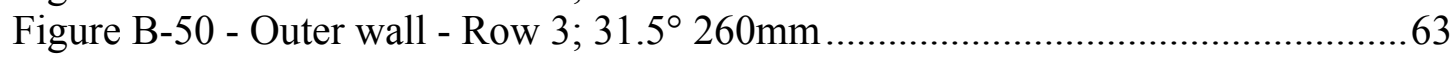

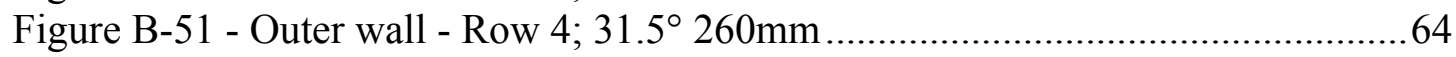

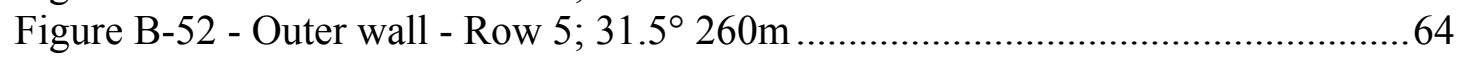




\section{TABLE OF TABLES}

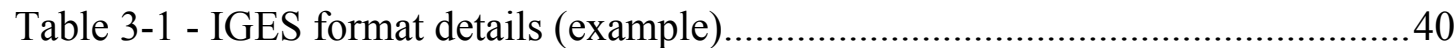

Table 3-2 - Traverse co-ordinates in CFX; 260mm deck height ................................45

Table 4-1 - Variance of probe coefficients with $95 \%$ confidence at $16 \mathrm{~m} / \mathrm{s}$................62

Table 4-2 - Continuity verification ........................................................................... 74

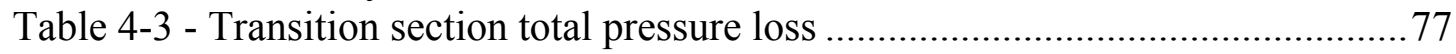

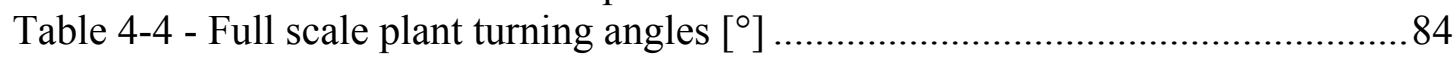

Table 4-5 - Full scale plant loss coefficient of transition section ...............................85

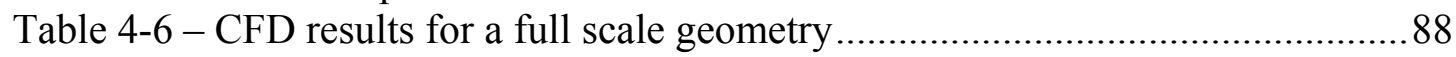

Table 5-1 - Experimental rig transition section performance ..................................8 89

Table A-1 - Separation and transition locations from edge of upper deck ................... 8

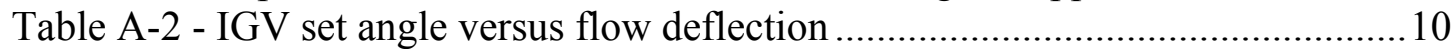

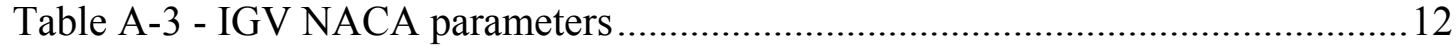




\section{NOMENCLATURE}

A

$\mathrm{B}_{\mathrm{F}}$

$\mathrm{C}_{\mathrm{L}}$

$\mathrm{C}_{\mathrm{P}}$

D

$\mathrm{H}$

$\mathrm{K}$

$\dot{m}$

$\mathrm{j}, \mathrm{i}$

$\mathrm{N}$

P

Q

q

$\mathrm{R}$

$\mathrm{S}$

V

$\mathrm{X}$

$\mathrm{v}^{*}$

$\mathrm{y}^{+}$

$\beta$

$\varepsilon$

$\theta$

$\mu$

$\varphi$

$\omega$

$\Lambda$

Area, through-flow area $\left[\mathrm{m}^{2}\right]$

Error of Function F

Loss coefficient based on a nominal chimney diameter, $\Delta$ Ptot /

$\left(1 / 2 \cdot \rho \cdot V^{2}\right)$

Coefficient of Pressure

Diameter, Chimney Diameter [m]

Collector height to chimney diameter ratio, h/D

Turbulence kinetic energy $\left[\mathrm{m}^{2} \cdot \mathrm{s}^{-2}\right]$

Mass flow rate $[\mathrm{kg} / \mathrm{sec}]$

Integer number

Integer, number, rotational speed [ $\mathrm{rad} / \mathrm{sec}]$

Pressure [Pa], Power [MW, kW]

Volume flow rate $\left[\mathrm{m}^{3} \cdot \mathrm{s}^{-1}\right]$

Dynamic pressure $[\mathrm{Pa}]$

Radius [m]

Variance, developed distance $[\mathrm{m}]$, blocked area $\left[\mathrm{m}^{2}\right]$

Velocity $[\mathrm{m} / \mathrm{s}]$, Volume $\left[\mathrm{m}^{3}\right]$

Variable

Wall friction velocity, $v^{*}=\left(\tau_{w} / \rho\right)^{1 / 2}$

Dimensionless inner law variable, $y^{+}=y \cdot v^{*} \cdot \rho / \mu$

\section{Greek symbols}

IGV stagger angle

Turbulence eddy dissipation [Velocity ${ }^{3} /$ length]

Probe attitude angle, turning angle into turbine

Viscosity $\left[\mathrm{m}^{2} / \mathrm{s}\right]$

Azimuthal angle of probe, turning angle at IGV tail

Turbulence frequency

Miscellaneous constant

\section{Acronyms}

ADDA Analogue-to-Digital-Digital-to-Analogue

AT

Auto Tran ${ }^{\mathrm{TM}}$

B

Bottom 
$\mathrm{BB}$

Below Bottom

CAD Computer Aided Drawing/Design

CFD Computational Fluid Dynamics

EFD Experimental Fluid Dynamics

F.S.O. Full scale output

HBM Hottinger Baldwin Messtechnik

IGV Inlet Guide Vane

M Middle

MTFM Matrix through-flow method

PC Personal Computer

RSM Reynolds Stress Model

SCPP SCPP

SST Shear Stress Transport

$\mathrm{T}$

Top, Blade row torque

\section{Subscripts}

$\perp \quad$ Perpendicular

ave Average

ax, a Axial

c The condition in a nominal chimney

rad, $r \quad$ Radial

S Static

tan Tangential

tot, $\mathrm{t} \quad$ Total

w wall

\section{Configurations for the SCPP Rig}
A
High deck (320mm); low IGV stagger angle $\left(22.5^{\circ}\right)$
B High deck; high IGV stagger angle (31.5 )
C Low deck $(260 \mathrm{~mm})$; low IGV stagger angle
D Low deck; High IGV stagger angle 


\section{CHAPTER 1:INTRODUCTION}

The main objective of this thesis is to determine the performance of the collector-tochimney transition section of a SCPP. The solar chimney power plant (SCPP) is unique in scale, but has a familiar layout, operation and function. For instance the collector-to-chimney transition section functions like an inverted Kaplan turbine (Von Backström and Gannon, 2004). Due to the SCPP's uniqueness and immaturity, there are currently no known applicable design methodologies for it and the tools to predict its performance are not firmly established yet. This chapter will introduce the reader to the SCPP, possible design methodologies and the objectives of this project. The rest of the thesis will establish more tools for the performance prediction of the SCPP.

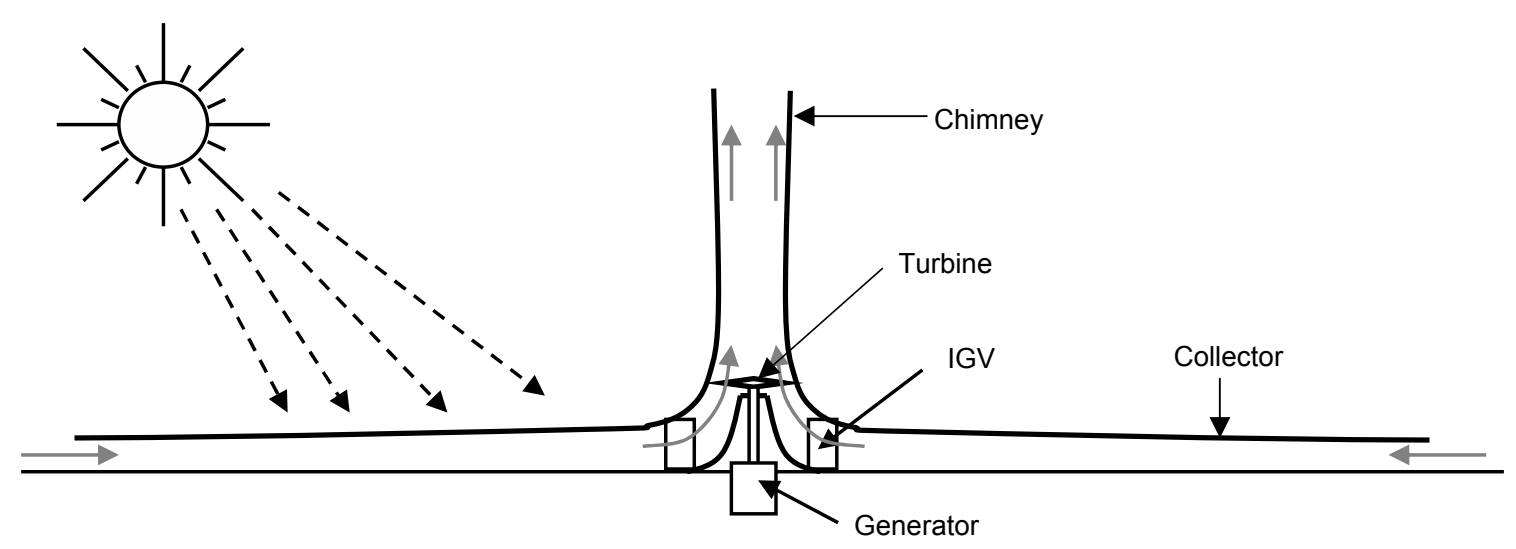

Figure 1-1 - Schematic illustration of a SCPP

\subsection{The Solar Chimney Power Plant}

Fossil fuels are non-renewable and produce emissions that are detrimental to the environment. Nuclear plants produce radioactive waste that is hazardous to store or dispose of. Such considerations have lead to the emphasis on 'clean' (non-polluting) energy. Energy sources that are considered clean are natural sources like wind, flowing water and solar radiation. Solar power has generally not been seriously considered as a source for the national power grid, due to the diurnal availability of sunlight. There is however a solution that is able to deliver power 24 hours a day. This solution is found in the SCPP. 
The principle of the SCPP is conceptualised by Leonardo Da Vinci (Haaf, 1983). His concept is a roasting spit that is powered by a turbine in a chimney above the fireplace. The turbine is in turn powered by the rising air from the hot coals. The SCPP, with its layout shown in Figure 1-1 and Figure 1-2, works similarly to the concept of Da Vinci's roasting spit.

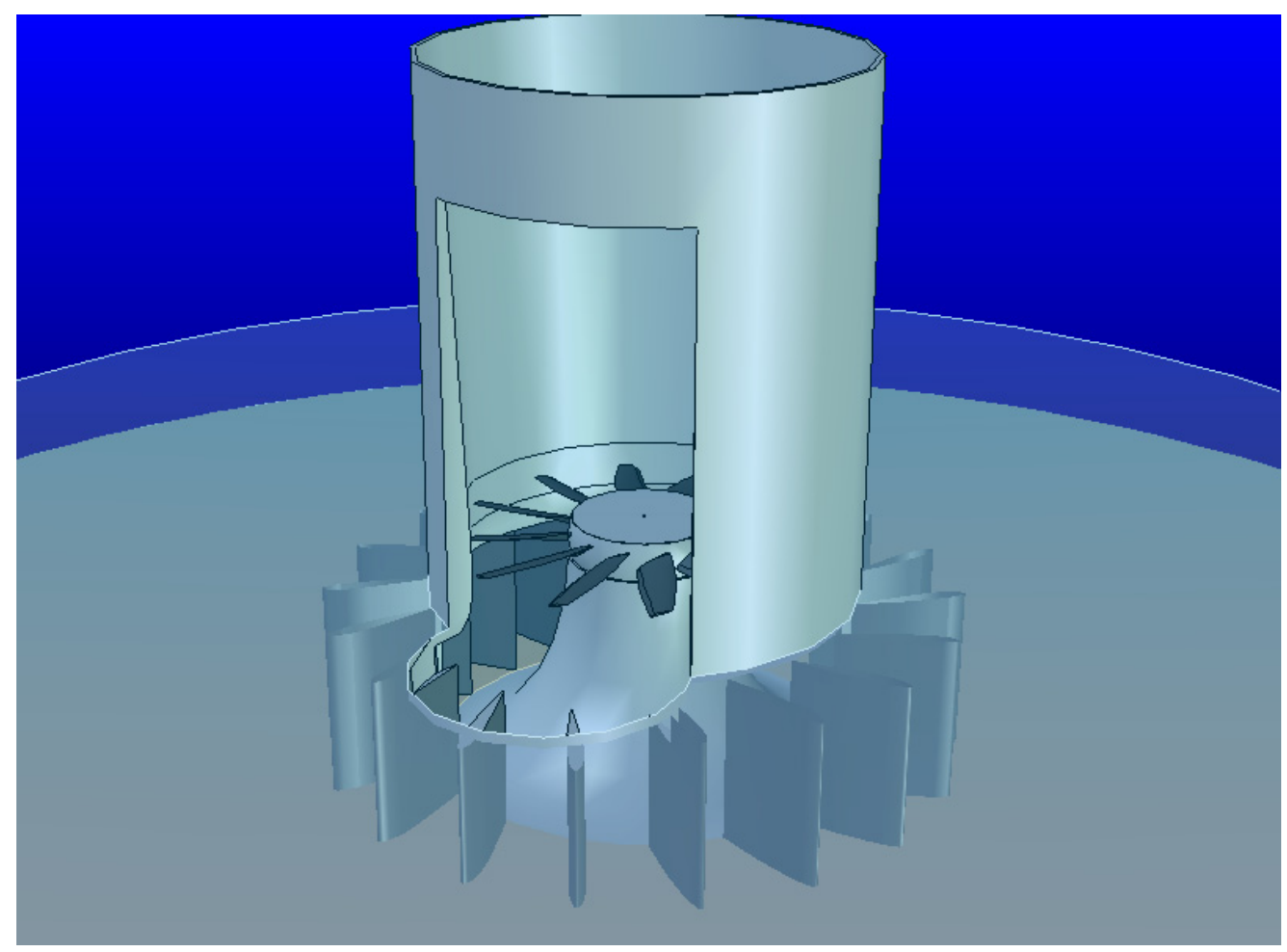

Figure 1-2 - Detail of the SCPP layout near its centre

The SCPP collects solar energy in the form of heat, which causes a density reduction of the air within it. The lower density air rises in the chimney, which causes the air to flow through the collector towards its centre. The air contracts through a transition section with inlet-guide-vanes (IGVs) into a chimney. The exit of the transition section is where a turbine-generator combination converts the driving potential of the air to electrical energy (Gannon and Von Backström, 2000).

To harness the solar radiation energy in the form of heat, a large circular collector is used. The collector consists of a glass roof (canopy) a few meters above the ground and is approximately $6 \mathrm{~km}$ in diameter for a 200MW plant (Gannon and Von Backström, 2000). Most of the short wave radiation (SWR) from the sun passes through the glass collector roof and is absorbed into the soil and stored in the form of 
heat. The heated soil emits long wavelength heat radiation (LWR) that does not pass through the glass. The net effect is that the air under the collector is heated as it flows towards the collector centre, decreasing its density and thereby expanding.

Figure 1-3 below illustrates this operation schematically.

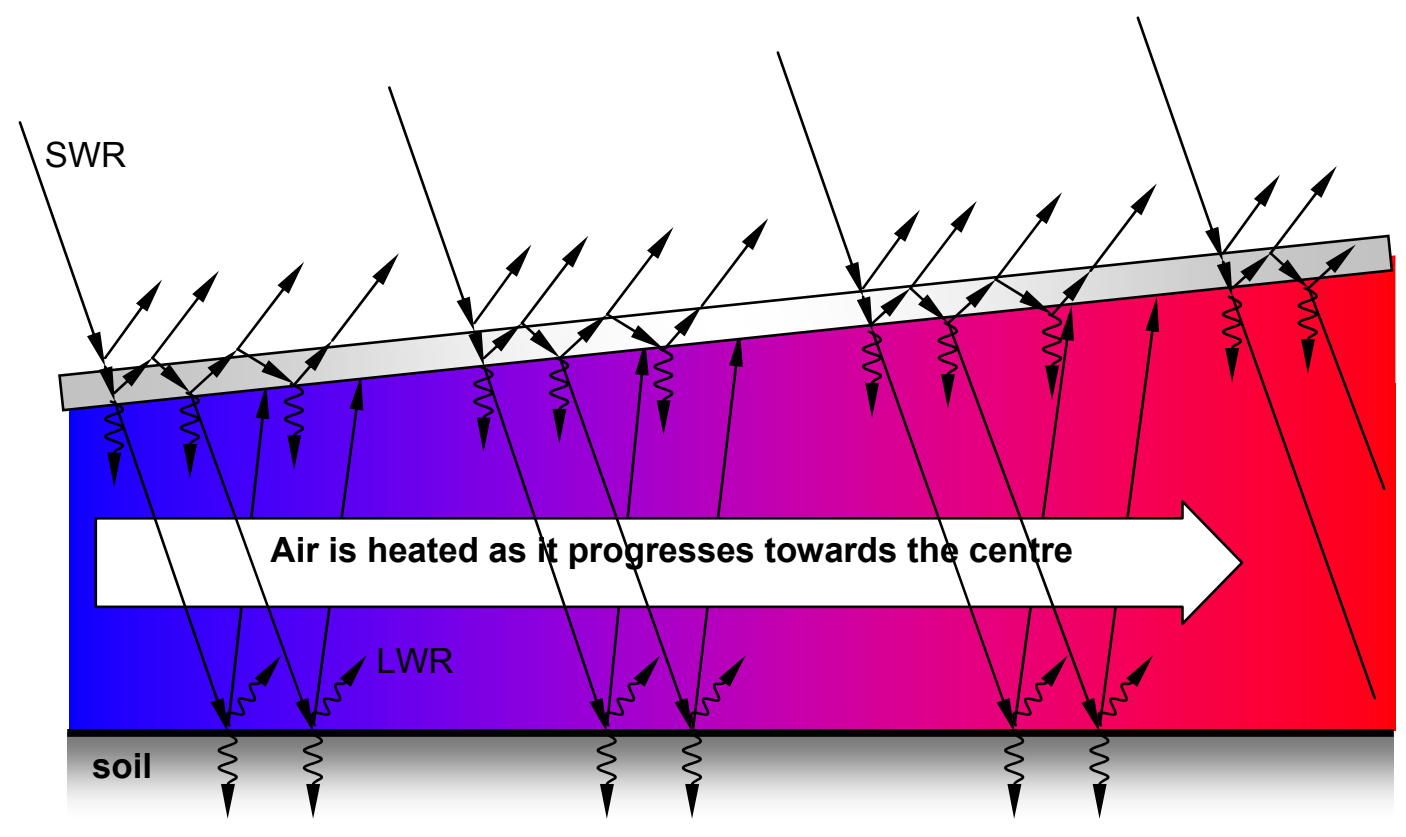

Figure 1-3 - Schematic of the heat transfer in the collector of a SCPP

Percentages of the short wavelength radiation (SWR) is reflected from the roof, refracted in the glass, emitted as long wavelength radiation (LWR or heat) into the air under the roof or transmitted to the soil.

Near its centre, the collector is approximately $60 \mathrm{~m}$ from the ground (Kröger and Buys, 2002). The concrete chimney is approximately $160 \mathrm{~m}$ in diameter with a mean wall thickness of one metre and a height of $1500 \mathrm{~m}$ (Von Backström, Bernhardt and Gannon, 2003). The weight of the chimney is supported by eighteen IGVs and the chimney itself is stiffened by internal spoked wheels (Schlaich, 1999 and Von Backström, Bernhardt and Gannon, 2003). The scale of the inlet section is illustrated in Figure 1-4 below relative to a Boeing 747.

Environmentalists may argue that in such large structure in which air flows of up to $250000 \mathrm{~kg} / \mathrm{s}$ occur (Von Backström, Kirstein and Pillay, 2003), the weather may be adversely affected. However, research to investigate the environmental impact of the SCPP has thus far not revealed any undesirable effects (Trieb et al., 1997). 


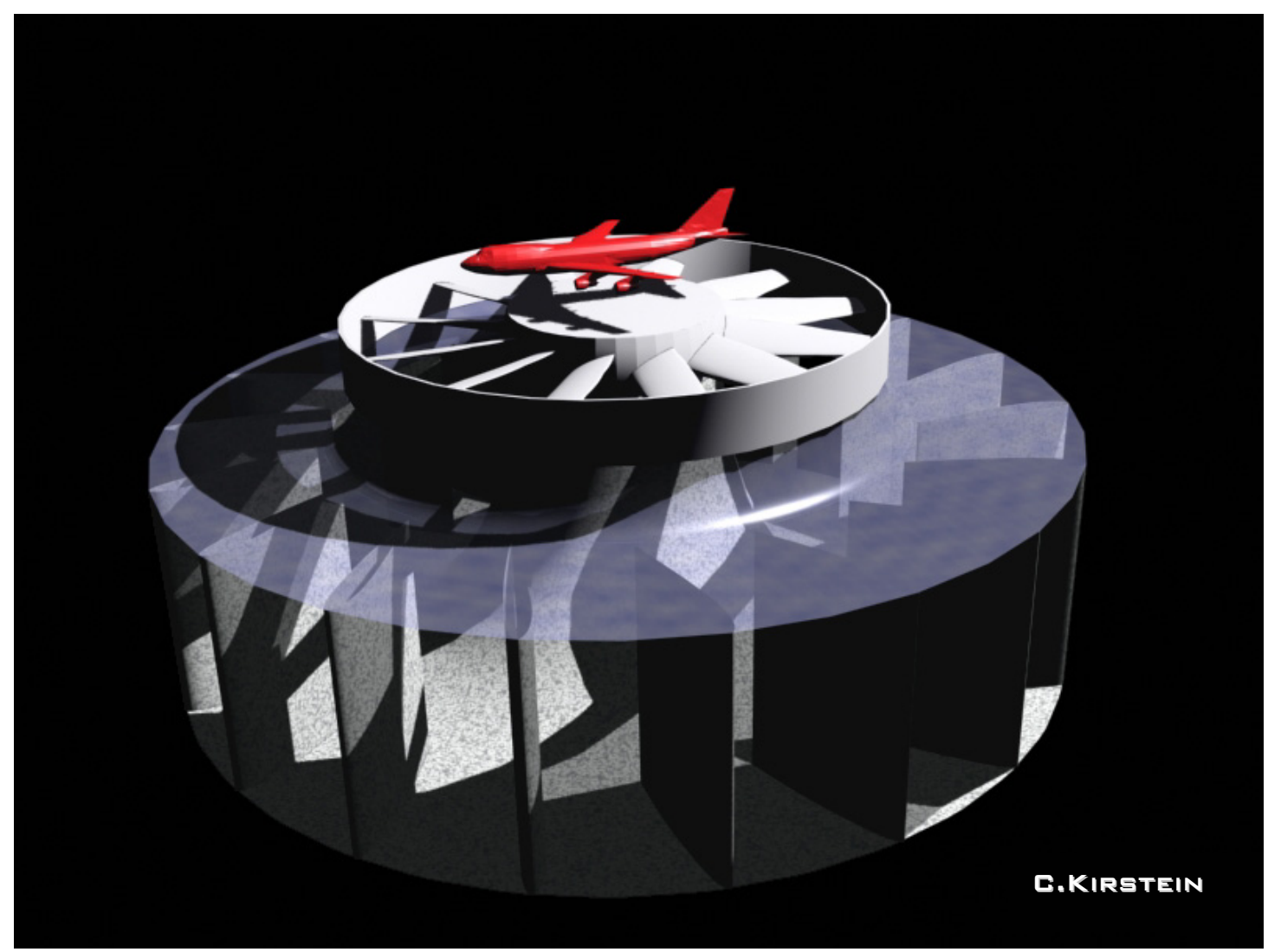

Figure 1-4 - Full scale transition section relative to a Boeing 747

A pilot plant of the SCPP was constructed and put to work at Manzanares in Spain in 1982. It had a $194.6 \mathrm{~m}$ high chimney with a $10 \mathrm{~m}$ diameter, a $244 \mathrm{~m}$ diameter collector and generated 50kW (Schlaich et al.; 1986). The SCPP functioned for seven years, and was fully automated the last three years. The pilot plant was able to deliver power $24 \mathrm{hrs}$ a day due to the thermal storage capacity of the soil (Haaf, 1984). Gannon and von Backström (2000) used this data to make predictions toward the full scale plant and found that the full scale plant would deliver power $24 \mathrm{hrs}$ a day as well, with a peak output of $200 \mathrm{MW}$ during the day and a minimum output of about $50 \mathrm{MW}$ at night. This pilot plant demonstrated the reliability and simplicity of the SCPP. The Manzanares pilot plant can be seen in Figure 1-5. 


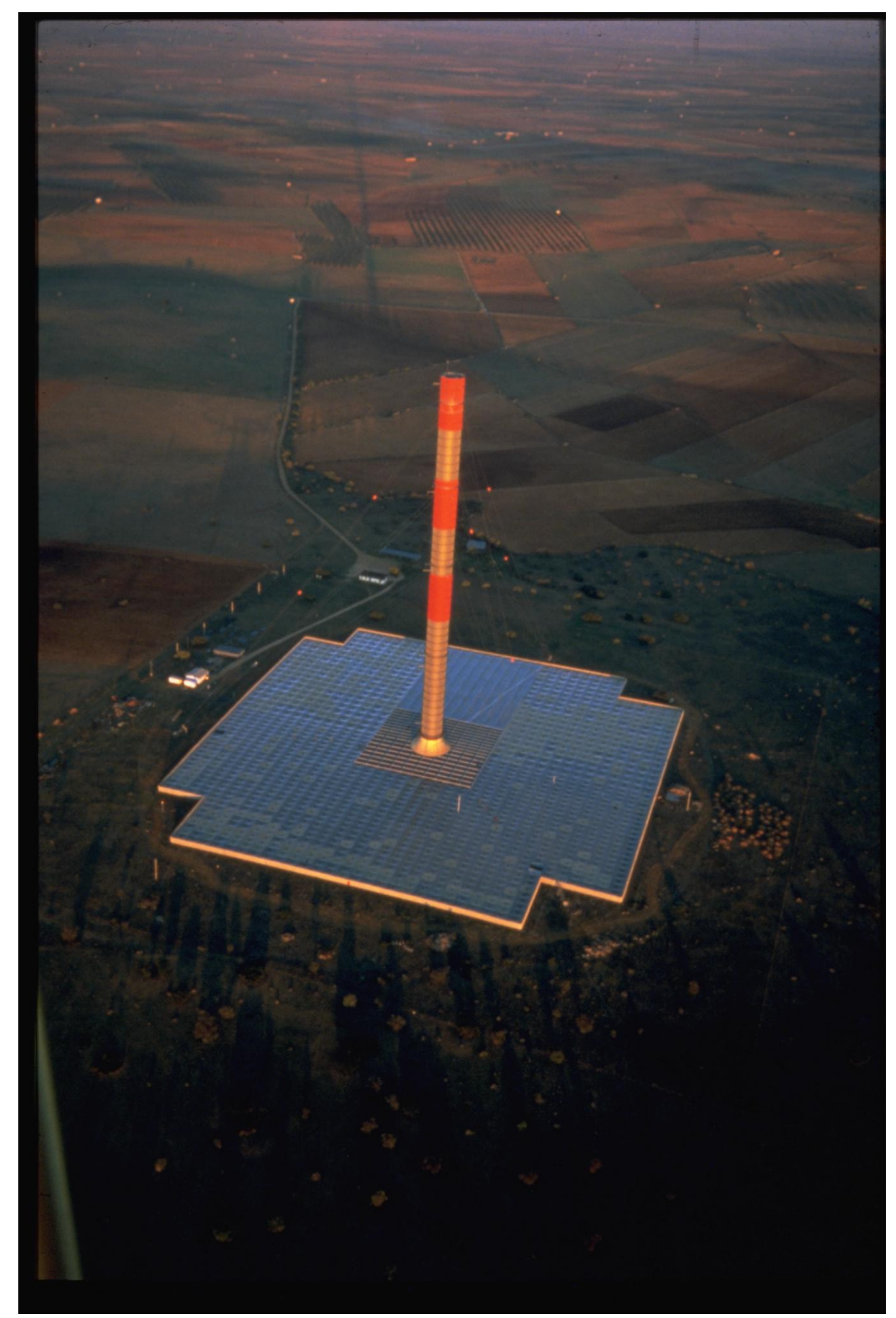

Figure 1-5 - Representation of the pilot plant at Manzanares

The major disadvantage of erecting a full scale SCPP is its capital cost. However, when the SCPP's layout is finalized and the erection method and manufacturing facilities established; the cost of producing each new plant would be less than the initial ones. Regardless, the capital cost will still be high in comparison to other power plants. Still, the main advantage of the SCPP over the other power plants is the 
operational costs. The pilot plant at Manzanares, for instance, was run by a local inhabitant trained on site (Haaf et al.; 1983). The SCPP would require some maintenance but in general, very few staff members and resources will be needed to operate and maintain the plant in comparison to any other power plant. Therefore the economical motivation is to have the chimney pay for its capital expenditure in a few years and from then on produce energy more economically than other existing power plants (Pasumarthi and Sherif, 1998b). Due to the large capital expenditure for a full scale plant, models that accurately predict a full scale plant's performance and characteristics have to be developed and established.

Pasumarthi and Sherif (1998a, 1998b), Gannon and Von Backström (2000), Kröger and Buys (2002) and Pretorius (2004) have developed theoretical models that estimate the SCPP's performance and characteristics. Gannon and Von Backström (2003), Von Backström, Bernhardt and Gannon (2003) and Von Backström, Kirstein and Pillay (2003) have acquired data from SCPP and turbine scale models.

Estimates for the loss coefficient through the collector-to-chimney transition section have been made by Kröger and Buys (2002) to be $\approx 0.25$ and by Gannon (2002) to be $\approx 0.163$ when based on the dynamic pressure of a nominal chimney diameter. Von Backström, Kirstein and Pillay (2003) refine Gannon's (2002) estimate to $\approx 0.161$ by taking the collector inlet loss coefficient into account. If the loss coefficient of the IGVs was excluded this estimate would have been $\approx 0.14$, but this thesis includes the IGVs with the transition section.

\subsection{ApPlicable Design Methodologies and Analysis ToOls}

The layout of the SCPP is that of an inverted Kaplan turbine, therefore the design methodology and analysis tools for Kaplan turbines could give further insight for the design considerations and analyses of the SCPP. What is especially sought after is information for analysing the transition section numerically and design methodologies for the meridional shape of the transition section.

The meridional shape of the SCPP transition section is similar to those of Kaplan turbines. The meridional shape of the transition section in the Stellenbosch SCPP model was designed to always contract up to the axial turbine. To achieve constant 
contraction the inner and outer walls of the duct have elliptical contours. This meridional shape was supposed to avoid adverse pressure gradients, but it did not. When it was analyzed by the matrix through-flow method (MTFM) of Gannon (2002), the analyses under predicted the wall static pressure on the elliptical part of the outer wall. The shape could not be corrected before experimental models were built since this numerical error was only discovered after the experimental data was acquired. De Jager (2000), Kirstein (2001) and Lebas (2001) could not improve these numerical predictions satisfactorily. Numerical prediction however seems generally to be problematic when investigating a radial-to-axial flow transition section in a Kaplan turbine layout.

Schilling et al. (1998) modelled a Kaplan turbine numerically, using air as the fluid in the experimental model instead of water. The numeric prediction of the velocity distribution through the section was mediocre and the pressure coefficients disappointing. Schilling et al. (1998) concludes that numerical simulation using a standard $k-\varepsilon$ turbulence model for complex 3D boundary layer flow along curved rotating walls where the cross flow due to swirl reaches the same magnitude of the main flow is not adequate to predict losses accurately. Schilling et al. (1998) therefore recommends that a more elaborate $\mathrm{k}-\varepsilon$ turbulence model in combination with an algebraic Reynolds stress model (ARSM) is developed and calibrated with precise measurements for this sort of application. This literature study concludes that there are currently no established tools available for the analysis of the transition section that are adequate. Since sufficient tools for analysis are not established, design methodologies for Kaplan turbines are investigated by comparing the SCPP transition section with the Kaplan turbine transition sections.

Extensive case studies of Kaplan turbines were documented in Jog (1976), Pylev et al. (1986), Lugaresi and Massa (1988), Magauer (1991), Roth (1991) and Zarea and Theodorescu (1997). Each of these indicates a similar curvature radius for the outer wall of the transition section when compared with the outer wall curvature of Gannon (2002). The relatively small curvature of the outer wall in the Kaplan turbine transition section could be explained by the angle of the inflow generated by the volute that leads the water through the IGVs. The volute generates prewhirl angles to the IGV row in the region of $60^{\circ}$ to $80^{\circ}$ to the radial direction, which is much larger 
than the almost completely radial inflow to the SCPP IGVs. The IGVs of the Kaplan turbine are not intended to change the flow angle significantly, and hence they are not specifically designed to turn the flow. Instead, for Kaplan- and Francis turbines, the adjustable IGVs have the purpose of loading or unloading the turbine by controlling the flow rate (www.tev.ntnu.no, 2003), not to generate swirl as with the SCPP. Turning angles cause the radius of curvature experienced by the flow along the outer wall curve to be lengthened by a factor of $\sec (\beta)$, where $\beta$ is the swirl angle. The $60^{\circ}$ to $80^{\circ}$ swirl angles at the exit of the Kaplan turbine IGVs makes the curvature gradient experienced on the outer wall substantially less than the $20^{\circ}$ to $35^{\circ}$ swirl angles of the SCPP.

IGVs are not intended for support in Kaplan turbines, but for the SCPP the IGVs support the entire chimney structure. Therefore the approach of adjusting the IGVs in the SCPP would not be similar to the Kaplan turbines, especially if the scale of the SCPP is taken into consideration. Furthermore the IGVs in the SCPP are currently designed to turn the flow from an almost completely radial direction; therefore only a part of the IGV has to adjust instead of the whole blade as with Kaplan turbines.

The differing swirl angles and functions of the IGVs in the SCPP and Kaplan turbines make the design objectives and approach somewhat unrelated. Therefore it is already a conclusion that the design methodology of a Kaplan turbine transition section would be inapplicable for the SCPP, especially due to the Kaplan turbine's much higher swirl angles. But for comprehensiveness the investigation is continued by exploring more considerations.

Detailed descriptions of Kaplan turbine design or dimensions are scarce in the literature. It appears that the designers use in-house relations, refined over the years by experience. Therefore there is no strict methodology for the design of Kaplan turbines. However the methodologies of various sources are compared to determine whether there is an applicable consistency.

The main dimensions of the Kaplan turbines have been investigated by Jog (1976), Pylev et al. (1986), Schweiger and Gregori (1987, 1989), Lugaresi and Massa (1988) and Zarea and Theodorescu (1997). All these cases have shown that the dimensions 
can be defined as functions of the specific speed. The sets of data are shown according the power specific speed defined by Sayers (1990), although this is essentially the same as the specific speed (§A.4.1). The power specific speed is defined as:

$$
N_{s p}=n \cdot P^{0.5} \cdot \rho^{-0.5} \cdot(g H)^{-1.25}=n \cdot P^{0.5} \cdot \rho^{-0.5} \cdot\left(\frac{\Delta p}{\rho}\right)^{-1.25}
$$

The dimensions that are of interest from these articles are the hub diameter of the runner, the IGV height and IGV location. Therefore these relations are compared in the figures below. The IGV height prediction of Schweiger and Gregori (1987) in Figure 1-7 is different to what the others recommend. This is probably because they have investigated Kaplan turbines with a steel casing instead of concrete like the others.

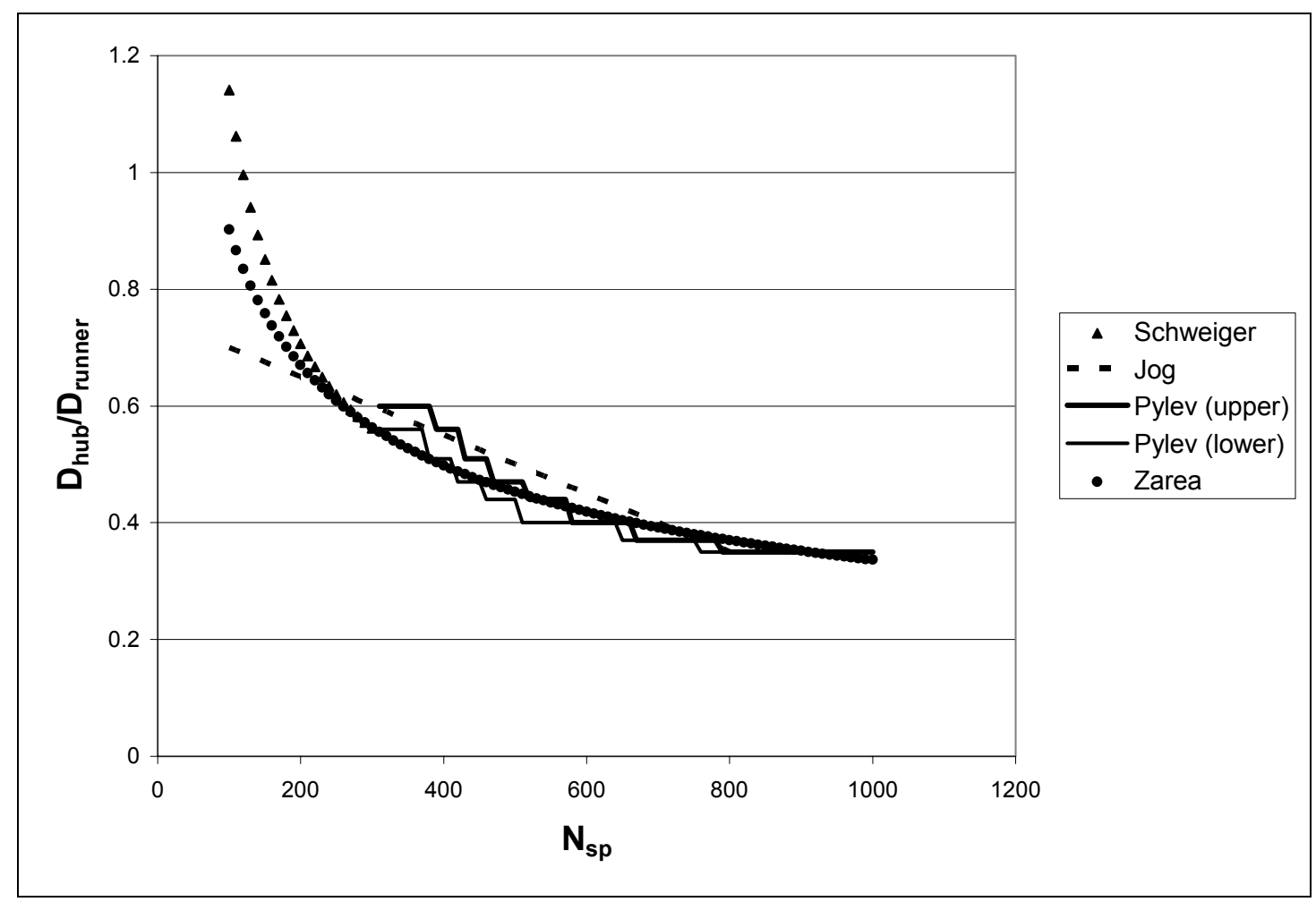

Figure 1-6 - Kaplan turbine hub diameter 


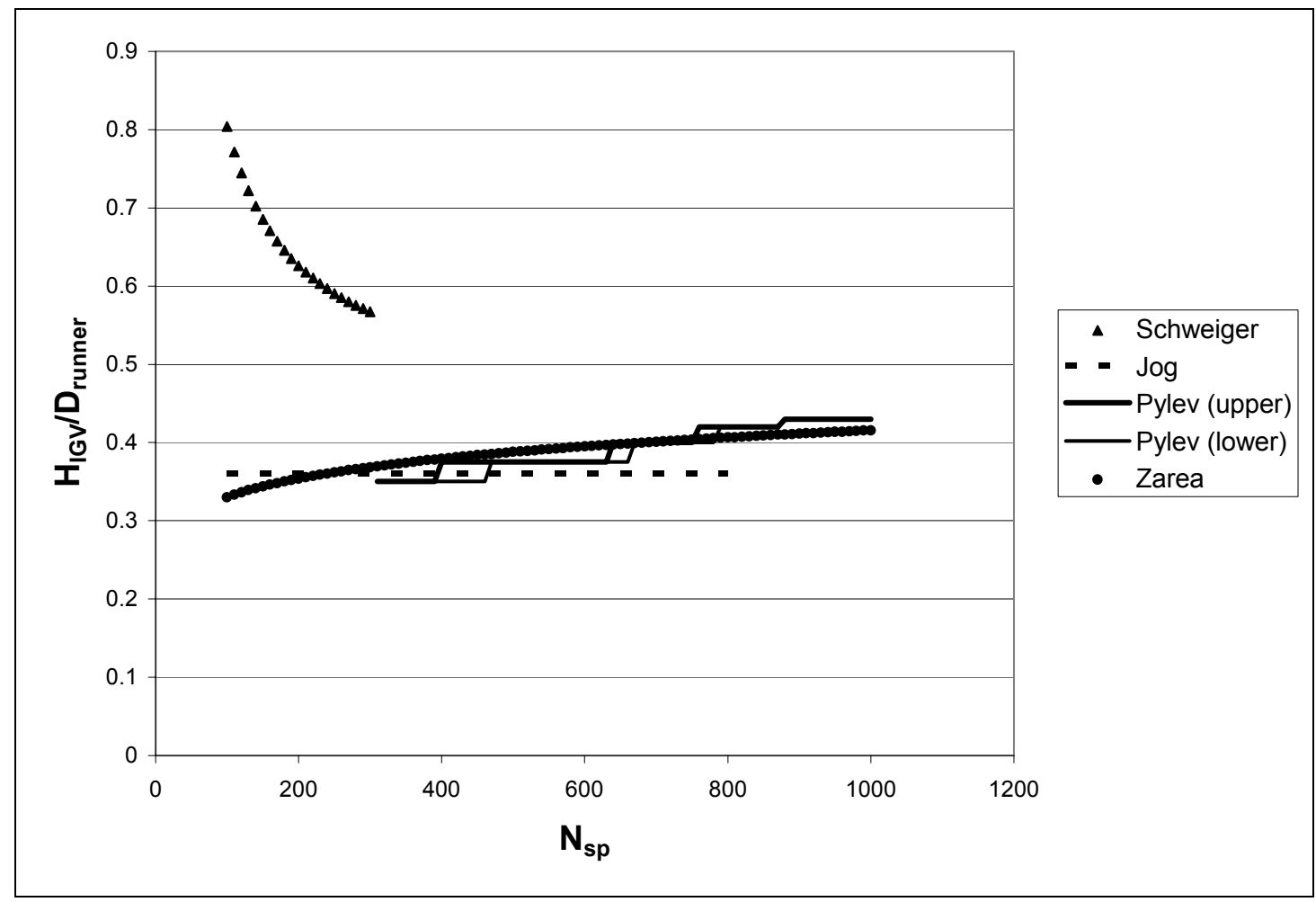

Figure 1-7 - Kaplan turbine IGV height

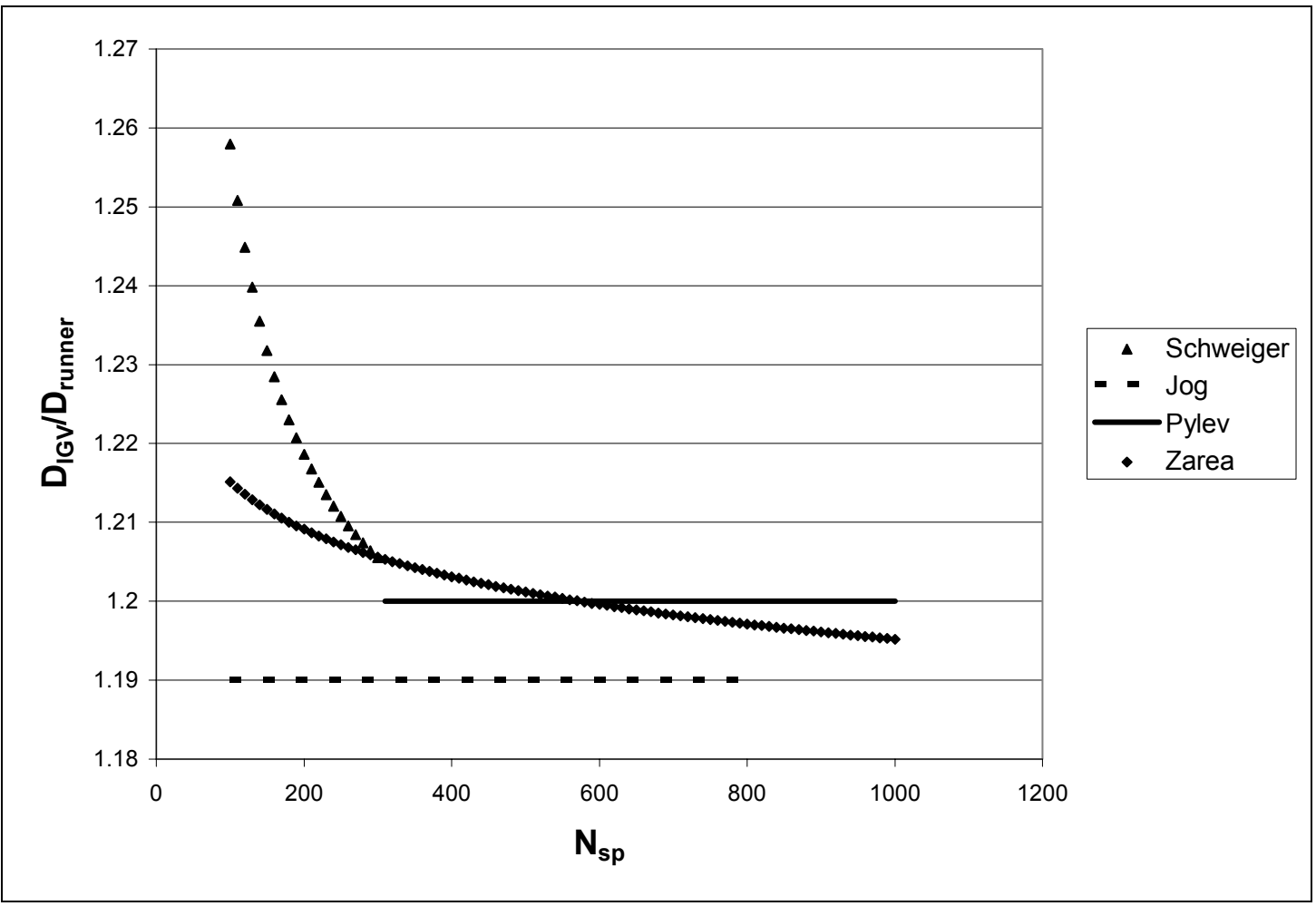

Figure 1-8 - Kaplan turbine IGV position diameter 
Two things can be observed from the figures above. First is that the in-house methods used to design the Kaplan turbine are subjective, but fairly consistent. Second is that the data are only applicable to the range of specific speeds in which they were specified. If the curves were extrapolated, the discrepancies would become more pronounced.

The range of these relations is $100<\mathrm{N}_{\mathrm{sp}}<1000$, except for the data of Zarea (1997) that can be extrapolated up to $\mathrm{N}_{\mathrm{sp}} \approx 13000$. The SCPP is expected to run at $15 \mathrm{rpm}$, deliver $200 \mathrm{MW}$, have pressure drop of $1000 \mathrm{~Pa}$ and an average density of about 1.04 $\left[\mathrm{kg} \cdot \mathrm{m}^{-3}\right]$ (Gannon and Von Backström, 2003 and Von Backström, Bernhardt and Gannon, 2003). This gives a power specific speed for the SCPP of $\mathrm{N}_{\mathrm{sp}} \approx$ $15 \cdot 2 \pi / 60 \cdot\left(200 \cdot 10^{6}\right)^{0.5} \cdot(1000 / 1)^{-1.25} \approx 3.95$. This is out of the range for the Kaplan turbine correlations shown in the figures above.

The conclusion is that the design methodologies that currently exist for Kaplan turbines are not applicable to the SCPP for the design of the transition section.

\subsection{ObJectives for this Project}

Now that the background to the SCPP and its transition section is known, the objectives for this thesis can be presented. Firstly, a list of the objectives is presented and then they are discussed in more detail afterwards.

The main objective is:

- To determine the performance of the transition section of the SCPP.

The secondary objectives are:

- To numerically model the flow behaviour in the transition section of the SCPP satisfactorily.

- To experimentally measure the flow behaviour in the transition section of the SCPP satisfactorily.

- To determine the effect alterations have on the strength and stiffness of the structure. 


\section{Transition Section Performance}

The performance for a small scale SCPP transition section will be determined by comparing numerical simulations and experimental data for various configurations of collector roof height and IGV stagger angle. Once the performance for the transition section of the small scale SCPP is determined, predictions on the performance of a proposed full scale plant can be made. The objective is to correlate the turning angle into the turbine and the loss through the transition section as dependent variables, and the collector roof height and IGV stagger angle as independent variables.

\section{Numerical Modelling of Flow Behaviour}

This thesis endeavours firstly to investigate, use and establish a sufficient numerical model to calculate the flow behaviour in the transition section. In this thesis higher order analyses will be conducted using a commercial CFD code.

\section{Experimental Measurement of Flow Behaviour}

The experimental data acquired thus far is incomplete to address the issues of axisymmetric behaviour, wake formations and losses through the transition section. This thesis will modify the current experimental rig to be able to measure the flow behaviour within the transition section for various configurations of collector roof heights and IGV stagger angles. This also means that any equipment errors will be identified and corrected so that the measured values are credible.

\section{Strength Alterations}

The chimney is carried by eighteen IGVs. Increasing the stagger angle of the IGVs causes a greater area of the IGVs to support the chimney. Increasing the angle of the inlet guide vanes also increases the torsional strength of the support structure. The magnitude of improvement in torsional strength of the plant is calculated when the IGV angle is increased with simple mathematical or numerical tools. 


\section{CHAPTER 2:EXPERIMENTAL FLUID DYNAMICS}

\subsection{INTRODUCTION TO EXPERIMENTAL MODELling OF THE SCPP}

To build a representative experimental setup or prototype early in the design phase is expensive. It is far more profitable to use computer- or mathematical models that are faster and simpler to modify (Blanchard and Fabrycky 1998). The problem with these models however is establishing their credibility. For CFD software especially, code validation has become a large subject of discussion and investigation (Roache, 1998; Rizzi and Vos, 1998; Oberkampf and Blottner, 1998; and Habashi et al., 1998). CFD is a useful tool in establishing flow behaviour, but when applied incorrectly or in unusual cases, the results are usually disappointing (Schilling et al., 1998).

It is helpful to establish credibility for CFD software by comparing it with experimental fluid dynamics (EFD). For a large plant such as the SCPP a full scale prototype would not be economically viable; therefore the EFD would also not be fully representative of the full scale performance. However once the flow behaviour is properly understood and the CFD and EFD are compared and calibrated accordingly (illustrated in Figure 2-1), then the prediction toward the full scale plant is more credible (Rizzi and Vos, 1998).

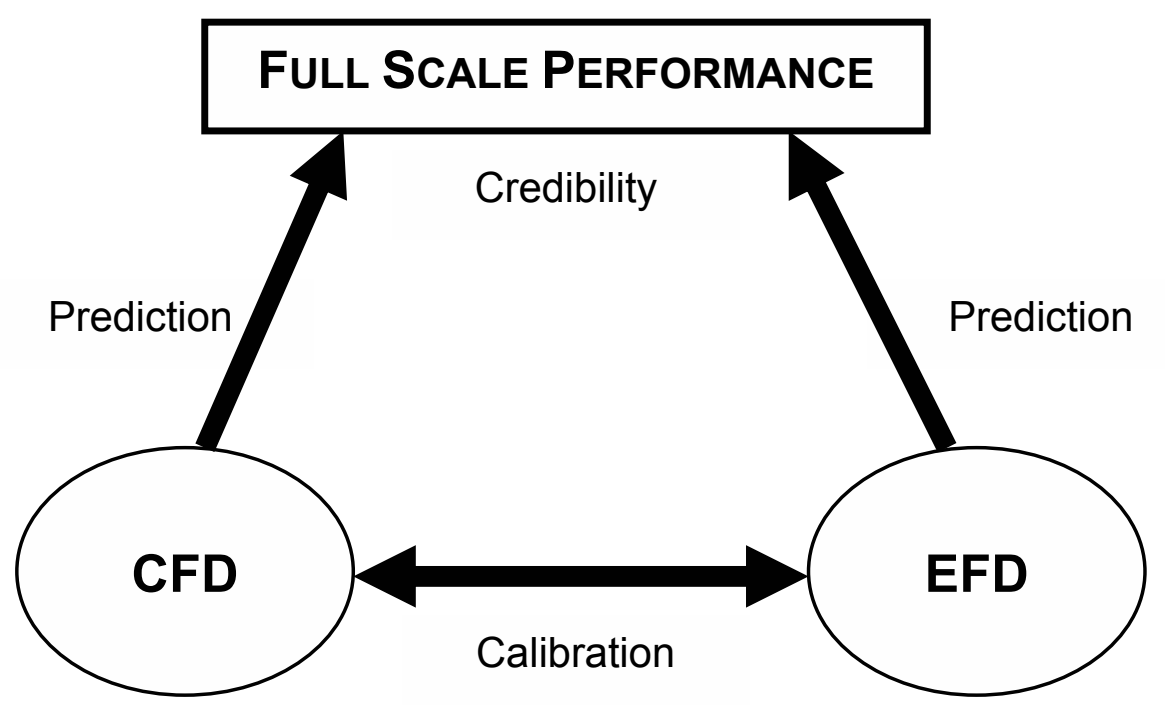

Figure 2-1 - Performance prediction and calibration by CFD and EFD 
The objective of the experiments is to facilitate establishing the credibility for the available CFD software to extend the predictions toward a full scale plant. The experimental representation of the SCPP for this project is a solar rig designed and built by Gannon (Gannon and Von Backström, 2003). This chapter describes the equipment and methods used to obtain the EFD results in chapter 4.

\subsection{EQUIPMENT AND INSTRUMENTATION}

\subsubsection{THE SCPP EXPERIMENTAL RIG}

The experimental rig only simulates the collector-to-chimney transition section of the SCPP, because it was designed and built particularly to determine the performance of the single turbine layout described by Gannon and Von Backström (2003). This thesis utilised the rig for investigating the losses through the section for the geometry currently proposed without the influence of the turbine.

The experimental rig is a 1:177 scale model of the reference SCPP plant in the Turbomachinery Laboratory of Stellenbosch University (seen in Figure 2-2). It is described by Kröger and Buys (2002) and built by Gannon (2002). Modifications to the rig were made by Kirstein (2001) to be able to evaluate the effect of independent variables on the performance of the plant. These modifications enabled four configurations that were used in this project. These configurations are:
A. High deck, low IGV stagger angle
B. High deck, high IGV stagger angle
C. Low deck, low IGV stagger angle
D. Low deck, high IGV stagger angle

The high deck is $320 \mathrm{~mm}$ and the low deck $260 \mathrm{~mm}$ from the collector floor, or $0.356 \mathrm{D}$ and $0.290 \mathrm{D}$ when normalised by a $900 \mathrm{~mm}$ chimney diameter. The low stagger angle is $22.5^{\circ}$ and the high stagger angle $31.5^{\circ}$ from the radial. These configurations are also in the nomenclature. 
Gannon (2002) designed the experimental rig with the purpose to keep the flow laminar up to the turbine, and this led to configuration A. Configuration $\mathrm{A}$ is the optimal configuration for performance and to prevent boundary separation for laminar flow. Gannon soon realised the performance could be improved since the flow was not laminar up to the turbine in configuration A. Hence Kirstein (2001) modified the experimental rig to obtain configuration $\mathrm{B}$ and $\mathrm{C}$ with his help. Configuration $\mathrm{B}$ and $\mathrm{C}$ are the optimal configurations for performance and to prevent boundary layer separation for turbulent flow according to criterion of Stratford (1959).

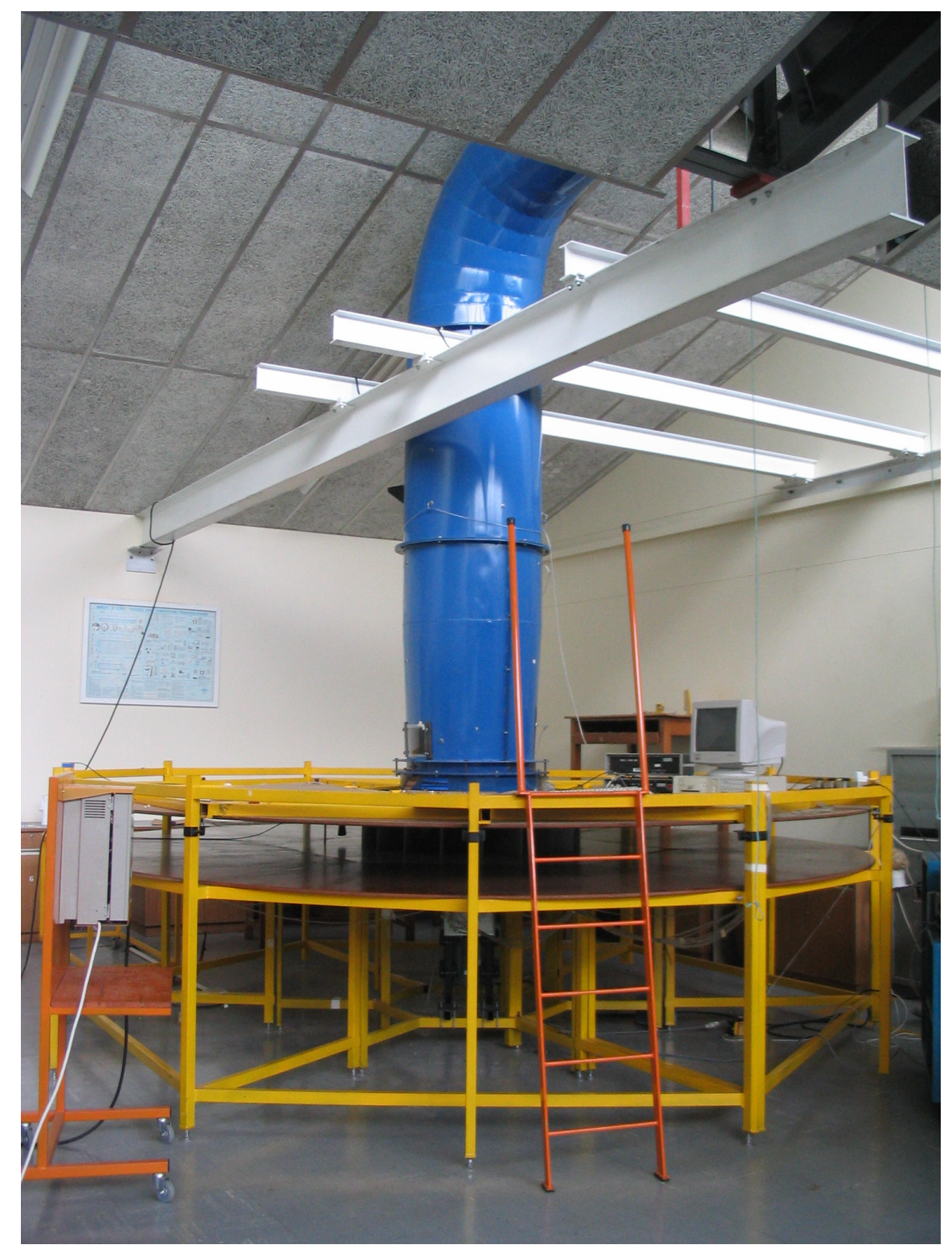

Figure 2-2 - SCPP Experimental Rig 


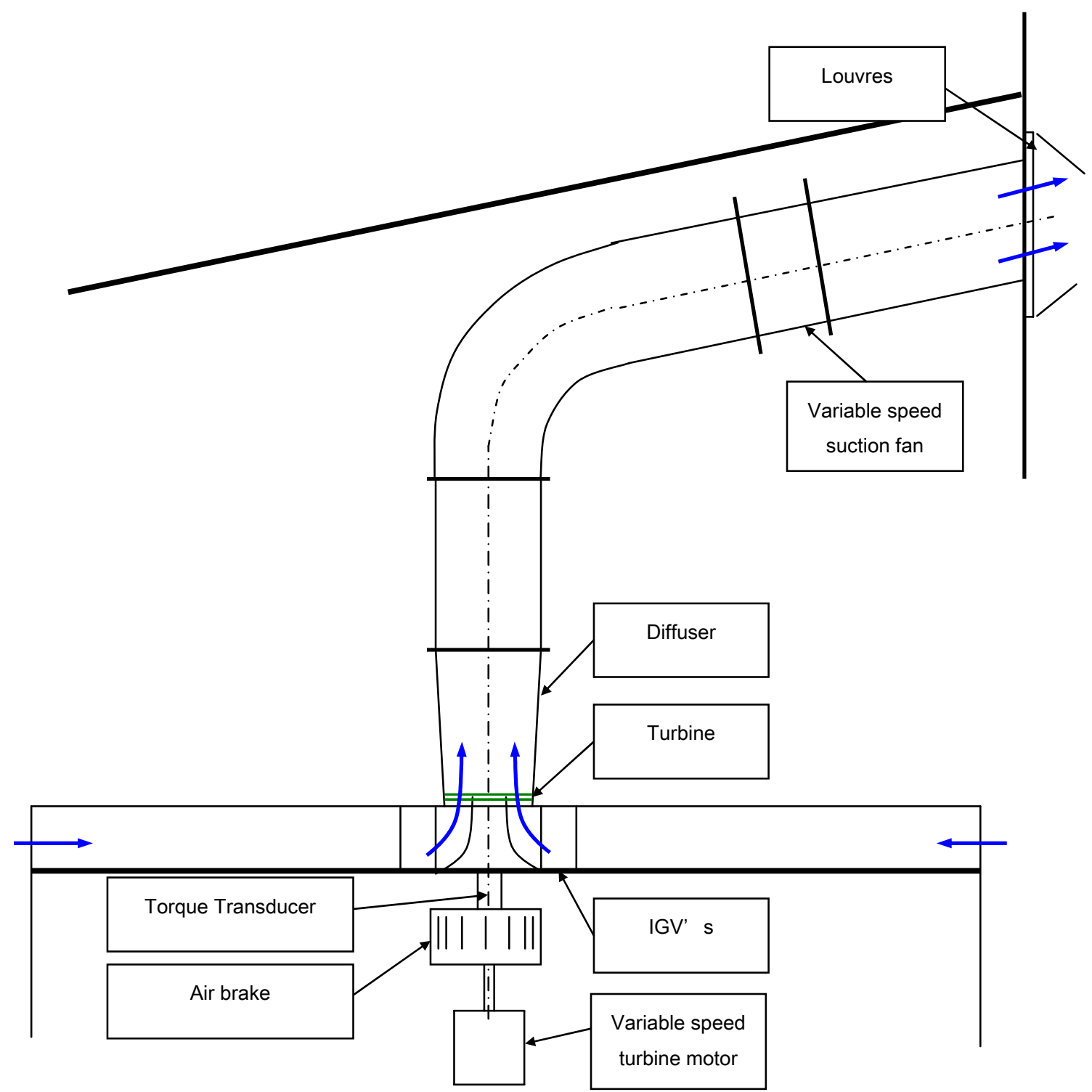

Figure 2-3 - SCPP Experimental Rig Schematic

\subsubsection{THE Five-Hole PROBE}

Measurement of flow in a known direction is accomplished by measuring the total and static pressures using a pitot tube. The pitot tube measures the total pressure with a hole on the stagnation point of the probe and the static pressure by pressure taps normal to the free stream. Measurement of flow of unknown direction is accomplished by using a probe with multiple pressure taps that are angled relative to each other. 
For complex three-dimensional flow, such as in the transition section of the SCPP, the angled pressure taps of a five-hole probe is ideal. The readings from the five holes can be combined to define a three-dimensional velocity vector as well as the static- and total pressure on the tip of the probe (Japikse, 1986). The figure below indicates the precursors of the angles and the method to obtain the required velocity vector at the probe head is explained in $\S 2.3$ on page 26 .

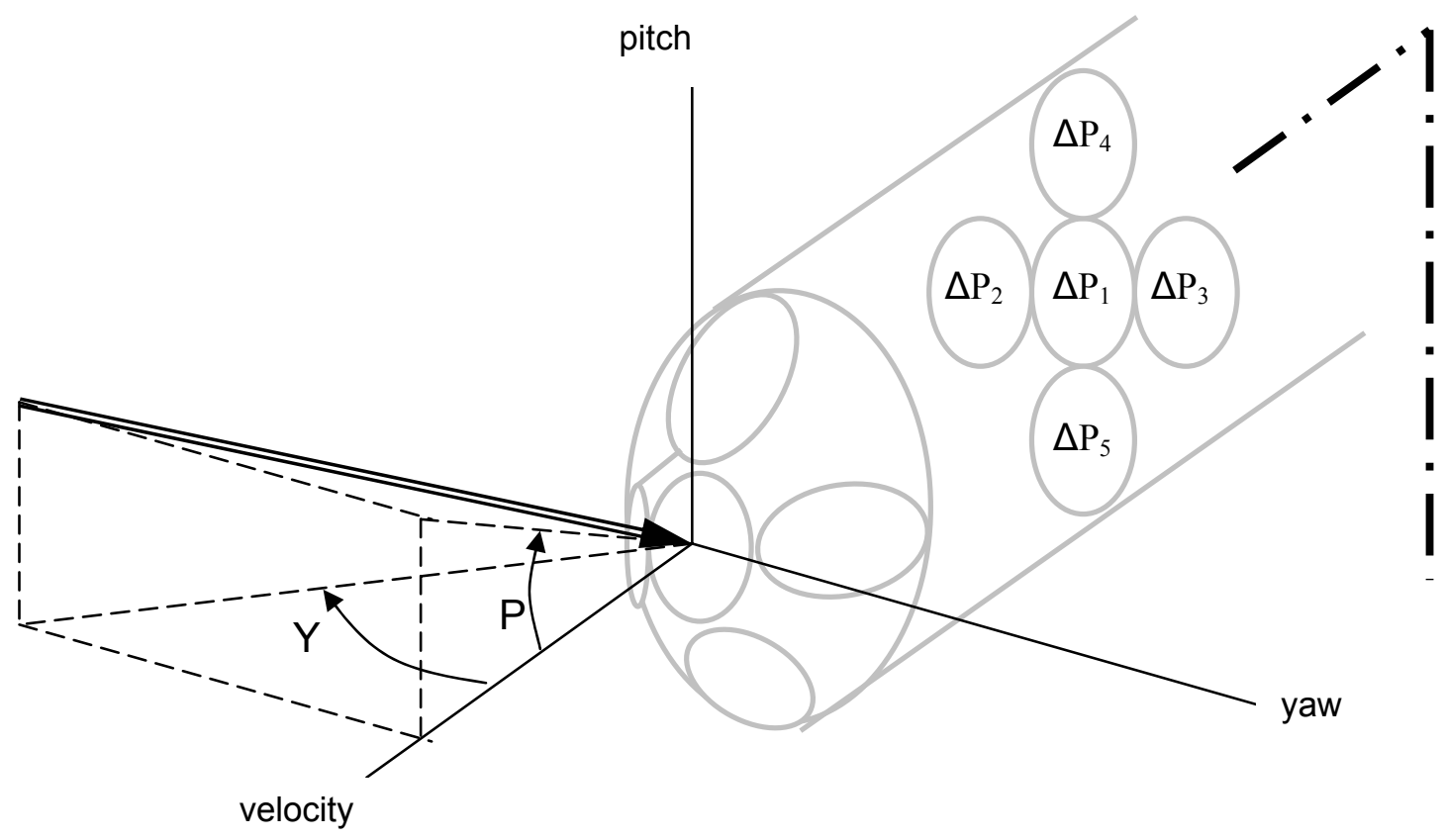

Figure 2-4 - Five-hole probe schematic

Figure 2-5 shows the size of the five-hole probe head that was used in this project. Its diameter is $1.4 \mathrm{~mm}$. The probe is small relative to standard probes, which enables it to measure the airflow characteristics without interfering significantly with the flow pattern and to get measurements near surfaces. The problem with the small bore tubes is that the probe takes relatively long to readjust and stabilize to a new pressure input when the probe position is adjusted, especially at lower velocities. 


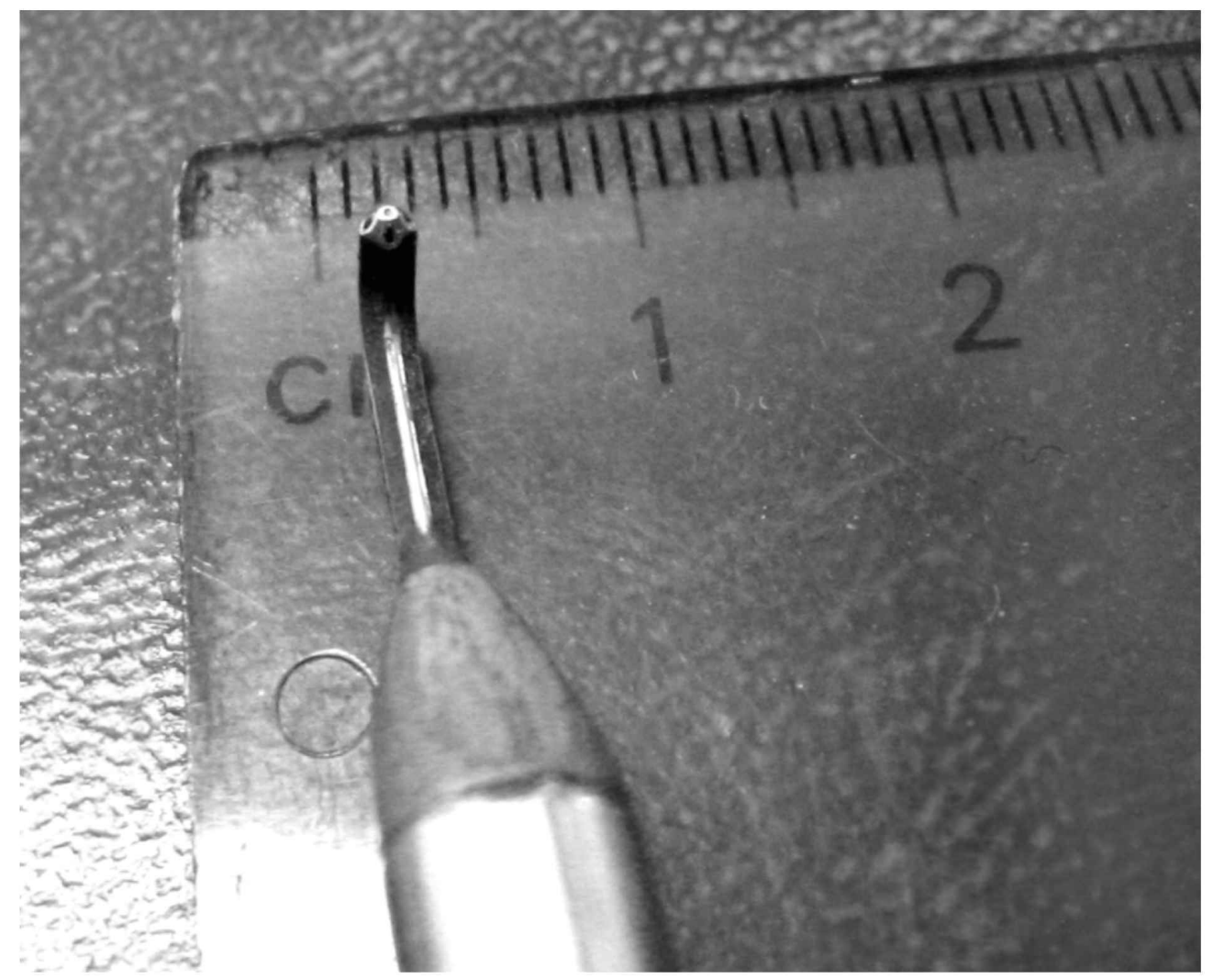

Figure 2-5 - Five-hole probe close up shown on a cm scale

The five-hole probe is mounted in a bracket that allows adjustment of the yaw angles and the traverse depth in the pitch-plane. If the magnitude of the pitch angle is too large for the probe to measure, then the velocity vector at that point cannot be defined. It can be defined for the yaw angle since the yaw angle of the probe can be adjusted until a reading can be obtained. This is however not possible for the pitch angle. For this reason it was very important to do a preliminary study on the flow field before the traversing vector was implemented in the experimental set-up. To do so threads that assist in visualising the flow and a numerical analysis were used beforehand to get an idea of the flow behaviour. The flow visualisation with threads is described in $\$ 2.5 .3$.

\subsubsection{HotTInger BaLdwin MesstechniK Pressure Transducers}

The Hottinger Baldwin Messtechnik (HBM) transducers have been used for all pressure measurements in the preceding theses relevant to this project. Hence it is important to be able to evaluate them accordingly. The HBM transducers are analogue transducers that are relatively simple to get readings from, since the signal is amplified by an analogue bridge amplifier. The transducers have a resolution of 
$0.1 \mathrm{~mm}$ water $(1 \mathrm{~Pa})$ and a $0.95 \%$ error on low pressure $(\sim 100 \mathrm{~Pa})$. Since they are amplified by an analogue amplifier, the setup requires recalibration at regular intervals during the experimentation. These transducers, however, were not used for the measurements in this thesis and merely served as a base for comparison to validate the AT transducers.

\subsubsection{HBM BRIDGE AMPLIFIER}

The bridge amplifier is an HBM DA24 with 5 usable channels, model KWS 3073. The input signal is shown on its display screen and sent to the PC via a connection to the ADDA card. The bridge amplifier was found to be sensitive to fluctuations in the power grid, although not as much as the AT transducers. The bridge amplifier, however, does have noise in general that makes the variance of the HBM transducers greater than the AT transducers. Furthermore the bridge amplifier is too sensitive for temperature variations and requires recalibration every 20 minutes. Therefore, as explained in more detail in $\$ 2.2 .3$ and $\$ 2.2 .5$, the HBM transducer and amplifier combination was not used for sampling pressures in this project and was only used to compare the performance of the AT transducers.

\subsubsection{AUTO TRAN ${ }^{\mathrm{TM}}$ 750D-250 PRESSURE TRANSDUCERS}

The Auto Tran ${ }^{\mathrm{TM}}$ (AT) pressure transducers were used for all experiments in this thesis for reasons that will become evident as they are discussed. One reason is that the lack of amplifiers enabled the whole data acquisition setup to migrate to on top of the experimental rig deck. These transducers calibrate themselves and require no additional amplification, they damp out noise and are easy to move around and set up. However, since these transducers have not been used before in the department, experimental verification was done to determine their credibility and characteristics. The transducers are specified by Auto Tran ${ }^{\mathrm{TM}}$ to have a lifetime stability of $0.5 \%$ full scale output (F.S.O.) and an accuracy of $0.75 \%$ F.S.O. To verify the transducers, firstly the accuracy then secondly the consistency of the transducers were compared with instrumentation of known characteristics.

The accuracy of the transducers was determined by comparing them with the readings of a Betz water manometer. This was done by connecting the Betz, AT transducer and 
my mouth by means of a T-junction and then acquiring the measured values. The figure below show the comparison, taking care to show what the clipping effect of the transducers is. Clipping is achieved when the transducer's maximum input pressure is exceeded. When a transducer clips, the output signal stays constant even though the input pressure increases. In Figure 2-6 the transducers clip at a pressure reading of 1664.5 $\mathrm{Pa}$ due to the set offset voltage. The output is from $1 \mathrm{~V}(0 \mathrm{kPa})$ to $10 \mathrm{~V}(2 \mathrm{kPa})$ with a zero offset value.

To obtain a pressure reading from the AT-transducer, the voltage offset value of the AT-transducer is subtracted from its output signal. The result is then divided by the gradient, which is $4.5[\mathrm{mV} / \mathrm{Pa}]$.

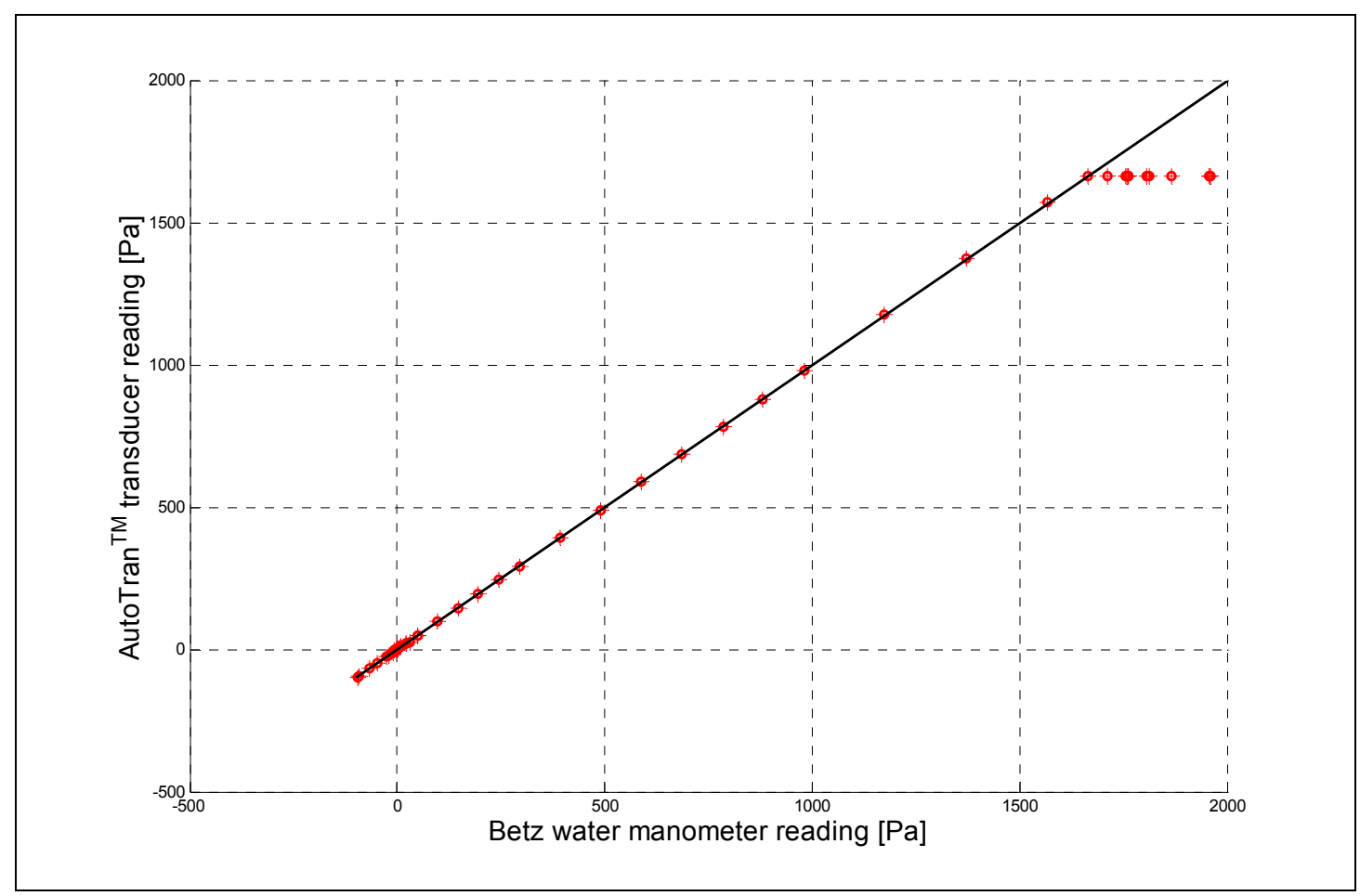

Figure 2-6 - AT Transducer validation

Pressure readings that are in range are extremely accurate as can be seen in Figure 2-6 and especially in Figure 2-7. The resolution of the transducers is approximately 0.55 $\mathrm{Pa}$, which is better than the specified resolution of the HBM transducers. The largest error over the full range is $2.2 \mathrm{~Pa}$, which is approximately $0.2 \mathrm{~mm}$ water. This is approximately the resolution of the Betz water manometer. From these results alone the AT transducer is entirely acceptable for all the purposes of this project, being approximately as accurate as a Betz water manometer. 


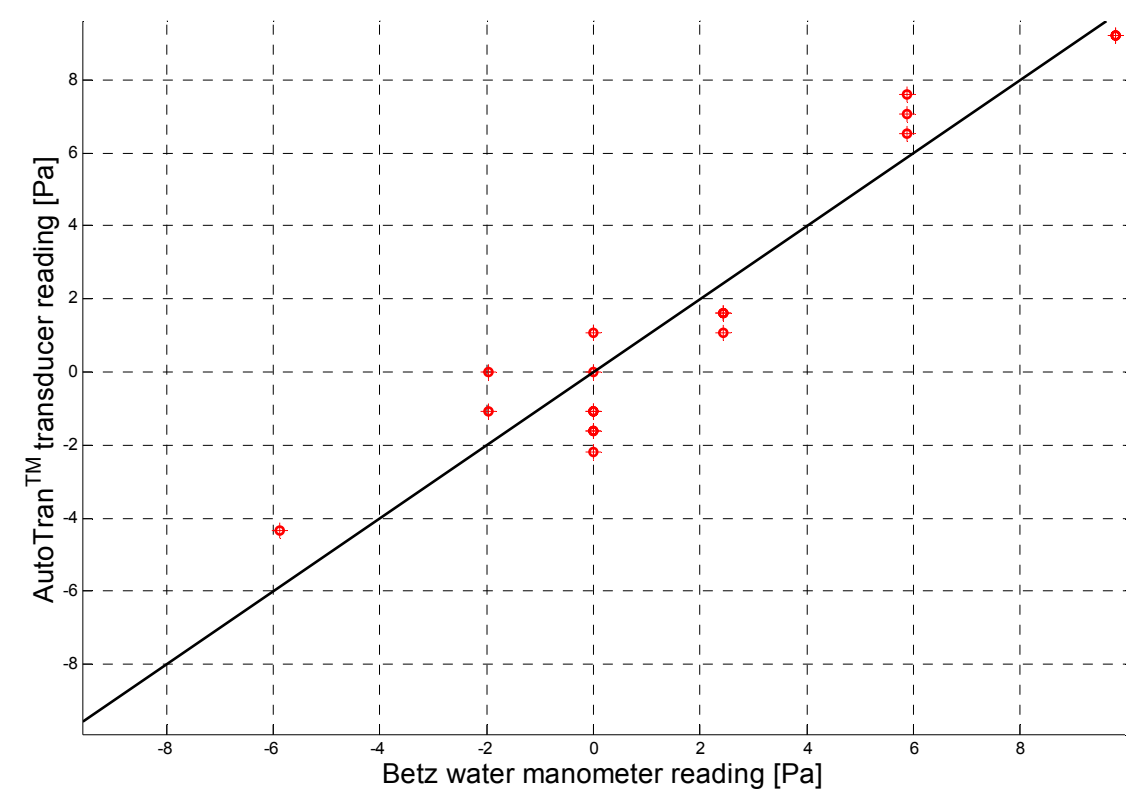

Figure 2-7 - AT Transducer validation zoomed

To determine the consistency of the AT transducers, they were compared with the HBM transducers. All instruments were calibrated and left to sample the pressure every 10 seconds for a few hours at a zero pressure input. This was done during the morning hours when the temperature increases by about $6^{\circ} \mathrm{C}$. The graph comparing the two sets of values over a selected time period is shown in the figure below.

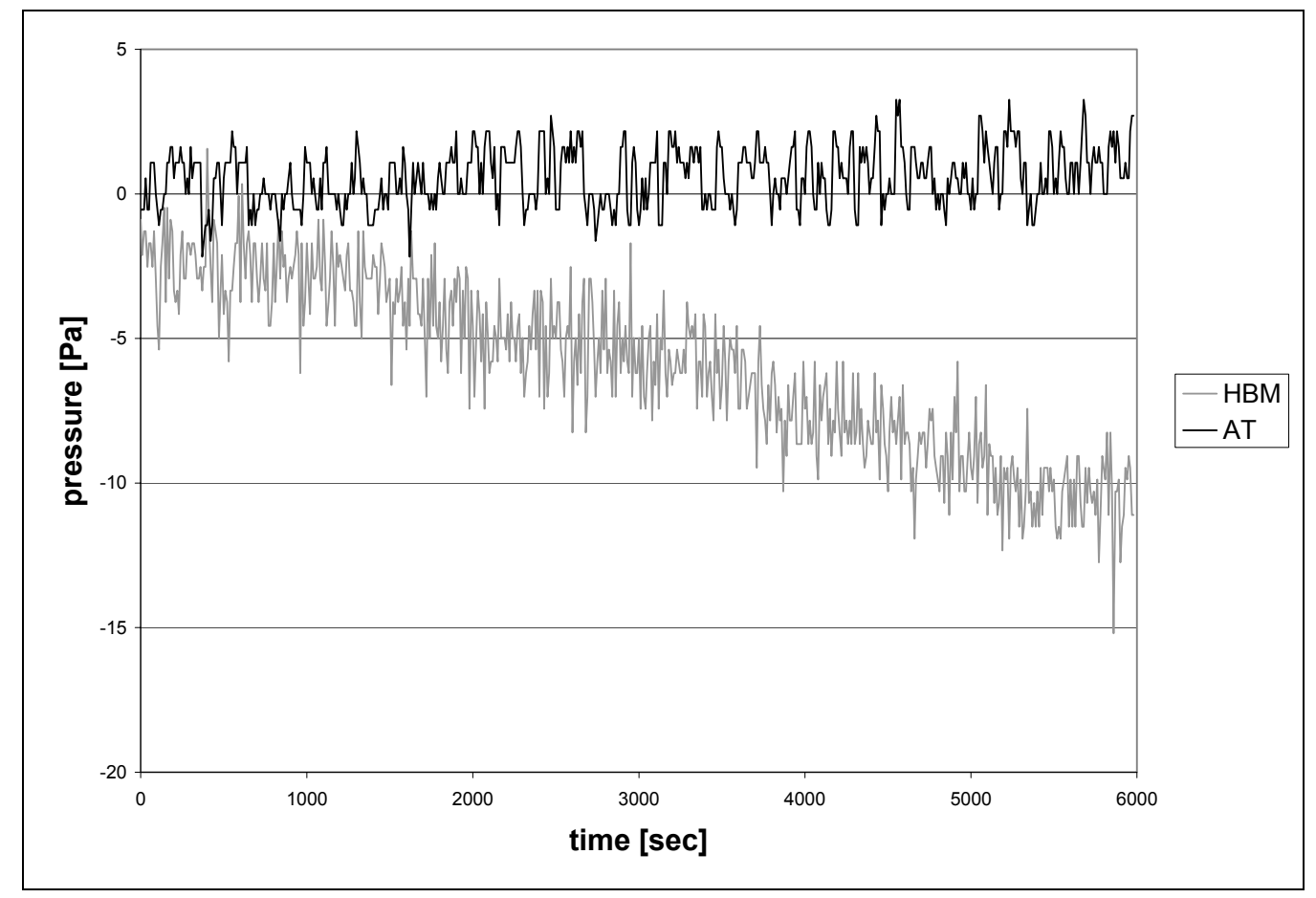

Figure 2-8 - Transducer variance comparison 
Clearly the HBM transducers have to be calibrated at least every 20 minutes to compensate for the drift, but the AT transducers are sufficiently stable. For this range of data the variance of the AT transducer was $1.725 \mathrm{~Pa}$ and the HBM transducer 2.241Pa, when compensating for the drift. Hence the variance of the AT transducer is lower than the HBM transducer, even when the correct zero position is used. Just to see how much the AT transducers would drift, the values of the calibration offsets were observed after two weeks since calibrating them. The offset pressure changed by $1.1 \mathrm{~Pa}$ in that time.

The problem noted with the AT transducers is that they are very sensitive to noise in the power grid, such as is generated by standard varispeed drives. The figure below illustrates the magnitude of the errors on both the HBM transducers and AT transducers when a varispeed drive is used. The HBM transducers are also affected by the noisy power grid, but clearly not as strongly as the AT transducers. This was one of the reasons the turbine could not be included in this project. The varispeed drives needs to be shielded from- or filtered for magnetic or electric fluctuations and this would have taken too long to implement.

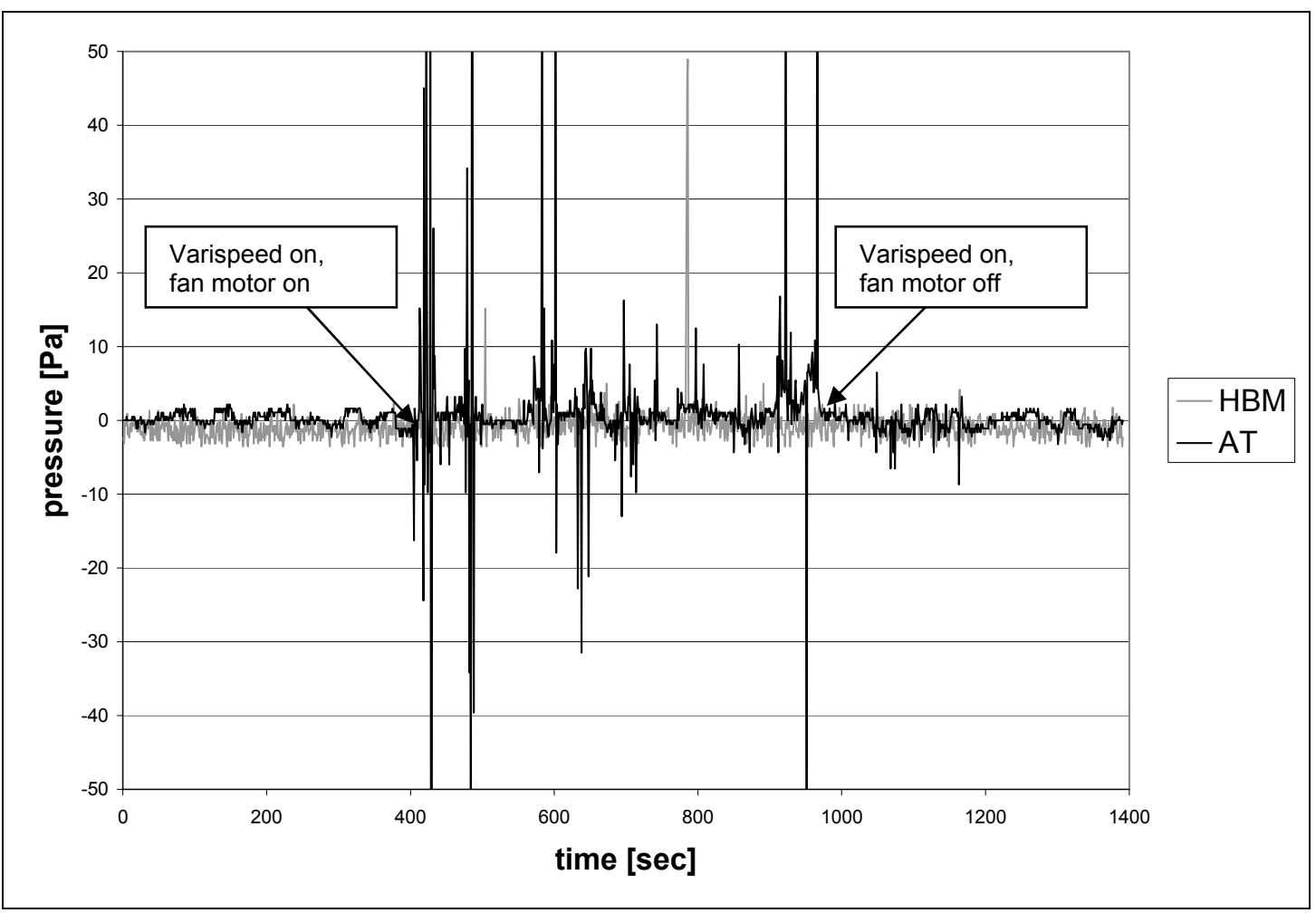

Figure 2-9 - Transducer sensitivity validation 


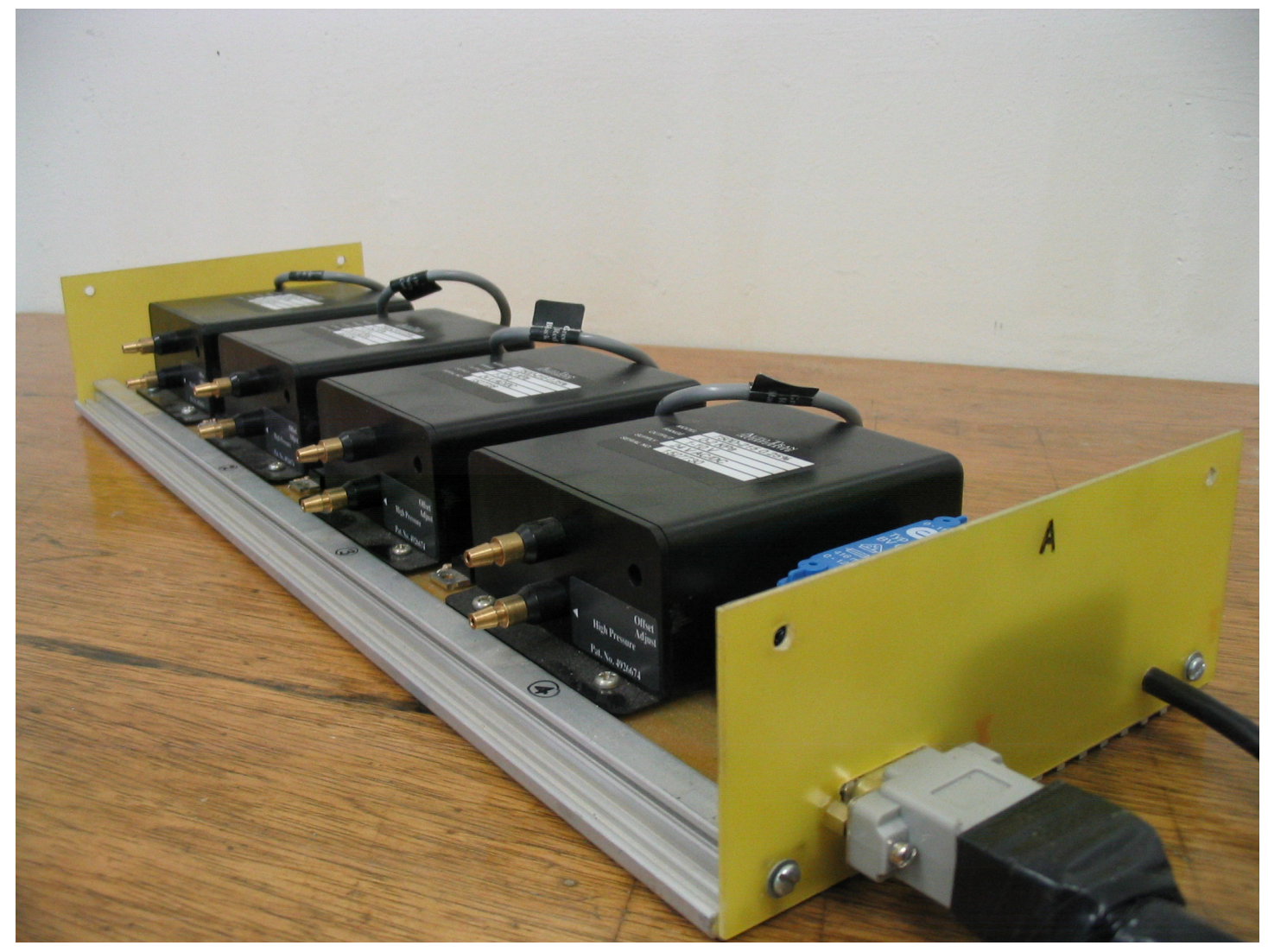

Figure 2-10 - AT Transducers

\subsubsection{PRESSURE SWITCH BoX}

The switch box is a FCO 91 MkII selection box. The switch box can have up to 40 channels with the slave box attached. The selection of the channels is controlled remotely via the ADDA card of the PC. Strohmaier (1997) describes the set-up in detail. 


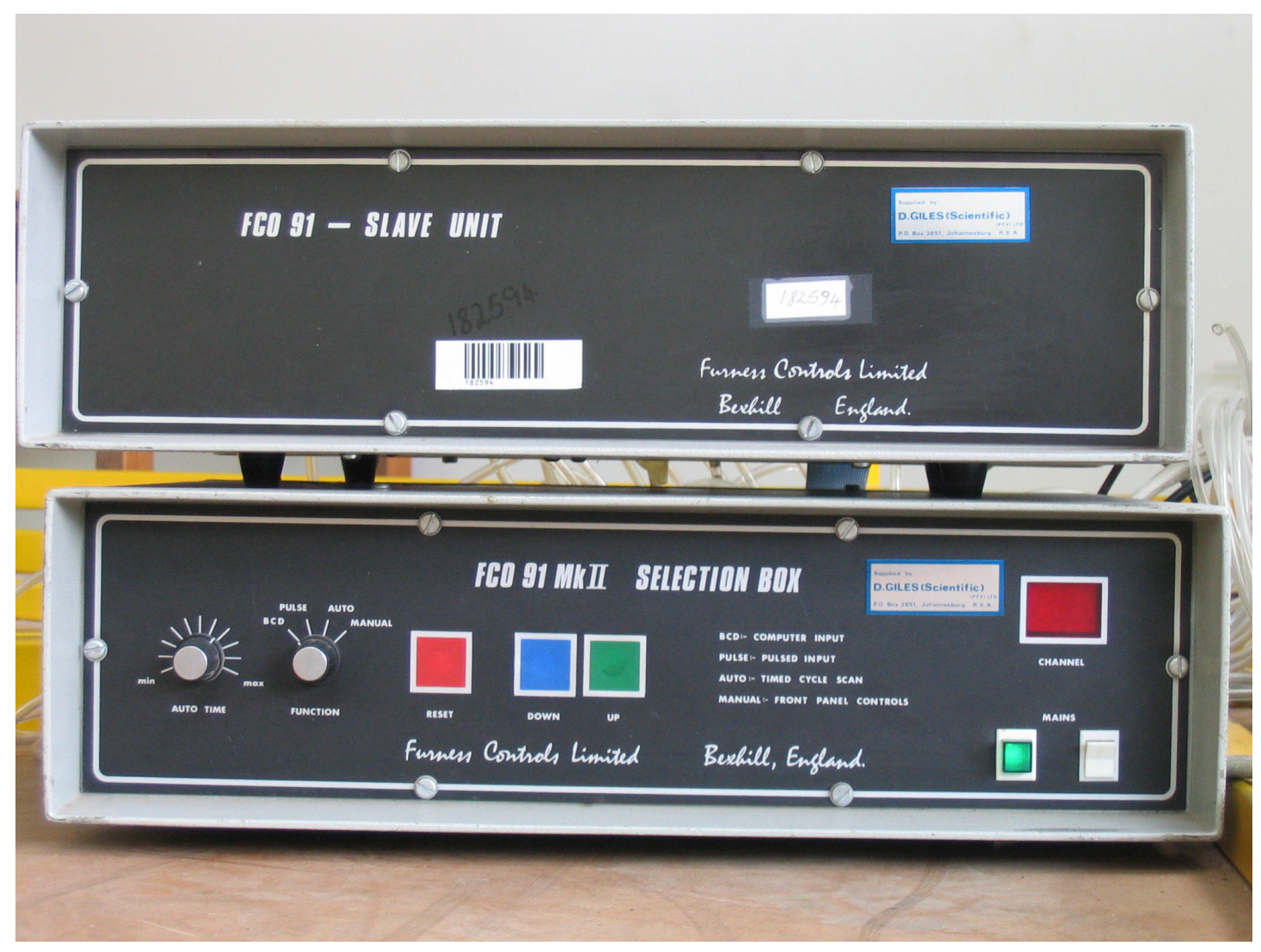

Figure 2-11 - Master and Slave Switch Boxes

\subsubsection{WINDMILL ANEMOMETER}

To measure the volume flow rate, a windmill anemometer is used. Gannon (2002) gives details on its calibration. The average error between the correlation used to determine the flow rate and the actual readings is supposed to be $2 \%$. However this was found to be marginally optimistic when the readings were taken.

The variance of one of its readings is shown in Figure 2-12 


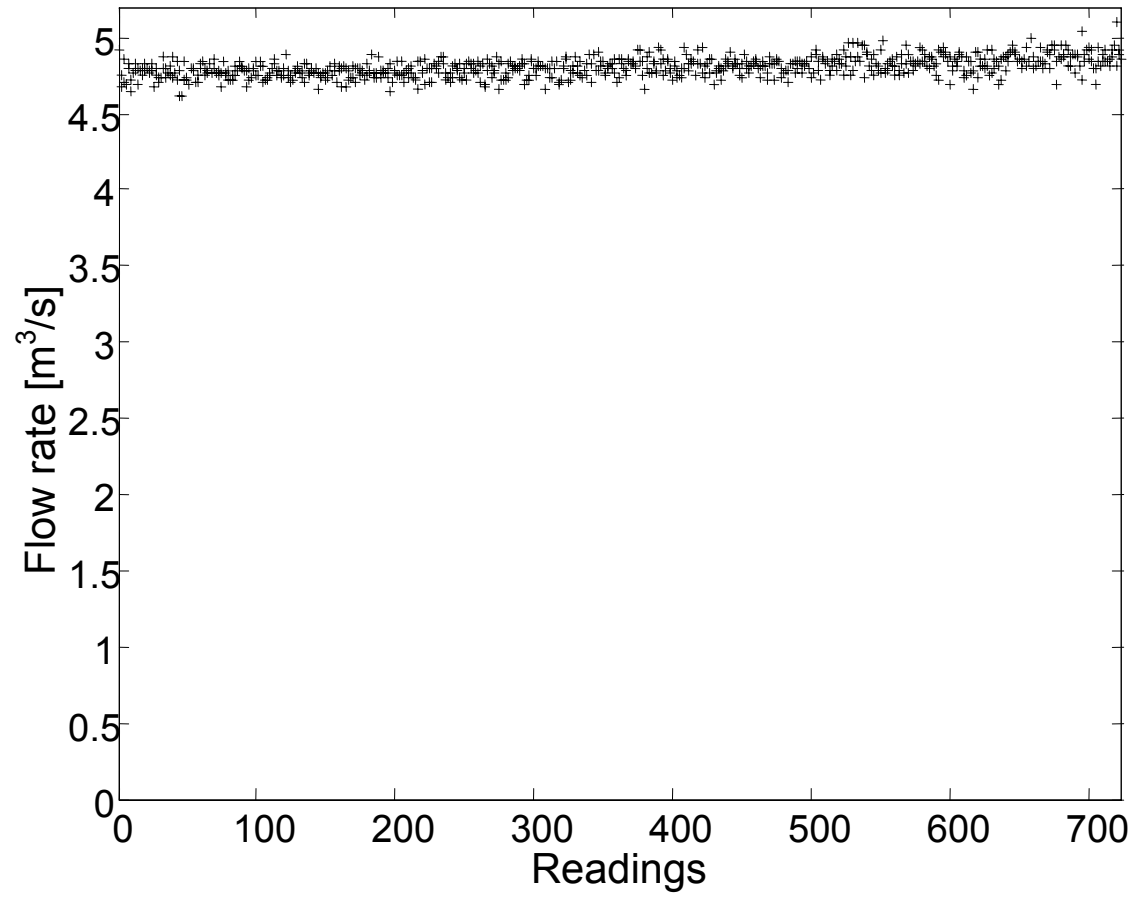

Figure 2-12 - Windmill anemometer flow rate $\left[\mathrm{m}^{3} / \mathrm{s}\right]$ readings $\left(22.5^{\circ} 320 \mathrm{~mm}\right)$

The anemometer takes 15000 samples each time a reading is taken. The variance is $0.1258 \mathrm{~m}^{3} \cdot \mathrm{s}^{-1}$, which is a $2.6 \%$ error in this case. Figure $2-12$ shows 724 readings of 15000 samples. The flow rate drifted slightly due to a changing temperature. The average is $4.8054 \mathrm{~m}^{3} \cdot \mathrm{s}^{-1}$, which under predicts the mass flow rate calculated from the five-hole probe. However, it was discovered that the anemometer had an offset error to the flow rate of $-0.153 \mathrm{~m}^{3} \cdot \mathrm{s}^{-1}$. Therefore this offset value is added to the readings that were taken in this project. This offset adjustment gave agreement with the fivehole probe for continuity to within $1 \%$ for the measured cases. The continuity comparison can be seen in Table 4-2 on page 74 .

\subsubsection{LAB COMPUTER}

The PC used to sample the data is a P100 with an Eagle technologies Analogue-toDigital-Digital-to-Analogue (ADDA) data acquisition card installed. The programs are written in Matlab ${ }^{\mathrm{TM}}$ v5 to control the switch boxes and to acquire the input voltages attached to the B-C-connector-panel. The sampling rate is approximately 3 $\mathrm{kHz}$, which is adequate for the type of experiments done in this thesis. 


\subsection{Five-hole PROBE CALibration}

\subsubsection{OBJECTIVE}

The five-hole probe described and calibrated by Strohmaier (1997), showed evidence of damage and the centre hole was partially blocked by particles. The probe was consequently unblocked and bent back into shape, but this caused the probe to be more sensitive to angular changes, making it necessary to recalibrate the probe. Secondly, compatibility problems with the new calibration maps made it necessary to implement a new sampling method into the existing program using these maps. This section will discuss the experimental procedure required and $\S 4.2$ the approach to both tasks.

\subsubsection{APPARATUS AND EQUIPMENT}

The required measurements for the experiment were:

- The pressure readings on each of the 5 holes of the five-hole probe

- A comparative pressure reading from a calibrated pitot tube.

- The true flow angles to compare with the measured flow angles

To obtain these values, the instruments are listed and then followed by detailed descriptions of the setup. The instruments required for this experiment are:

- Five-hole probe

- small wind tunnel

- standard pitot tube

- two Betz water manometers

- AT pressure transducers for each of the channels

- PC with ADDA card

- Adjustable mounting bracket for probe in small wind tunnel

The setup for the five-hole probe in the mounting bracket is shown in Figure 2-13. The bracket is screwed on top of the small wind tunnel and allows pitch adjustments of $5^{\circ}$ increments. The five-hole probe bracket allows yaw angle adjustments of $1^{\circ}$, but for symmetry in the maps only $5^{\circ}$ increments were used. 
Figure 2-14 shows the setup of the five-hole probe in the wind tunnel with the pressure transducers and lab computer in place. The arm that enters the side of the tunnel holds the pitot static tube, which is connected to the Betz water manometers. One Betz measures the total pressure and the other the static pressure.

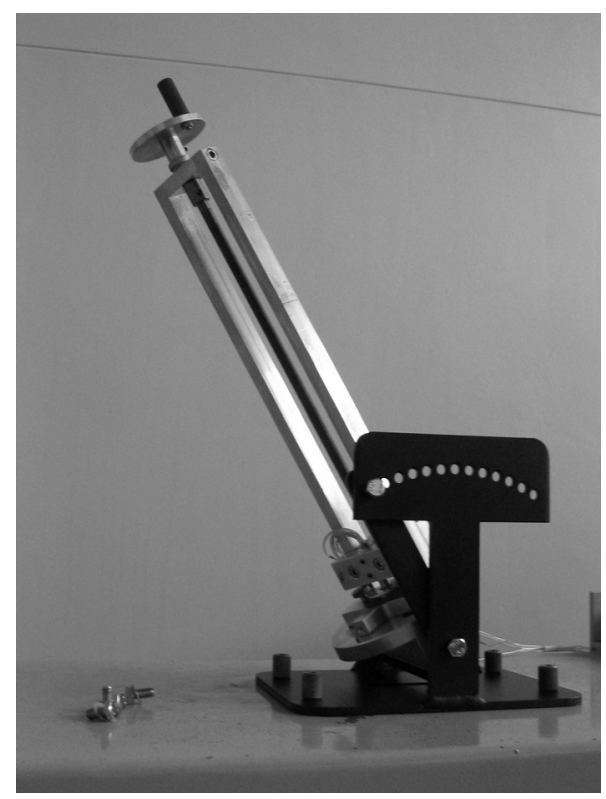

Figure 2-13 - Five-hole probe calibration mounting detail

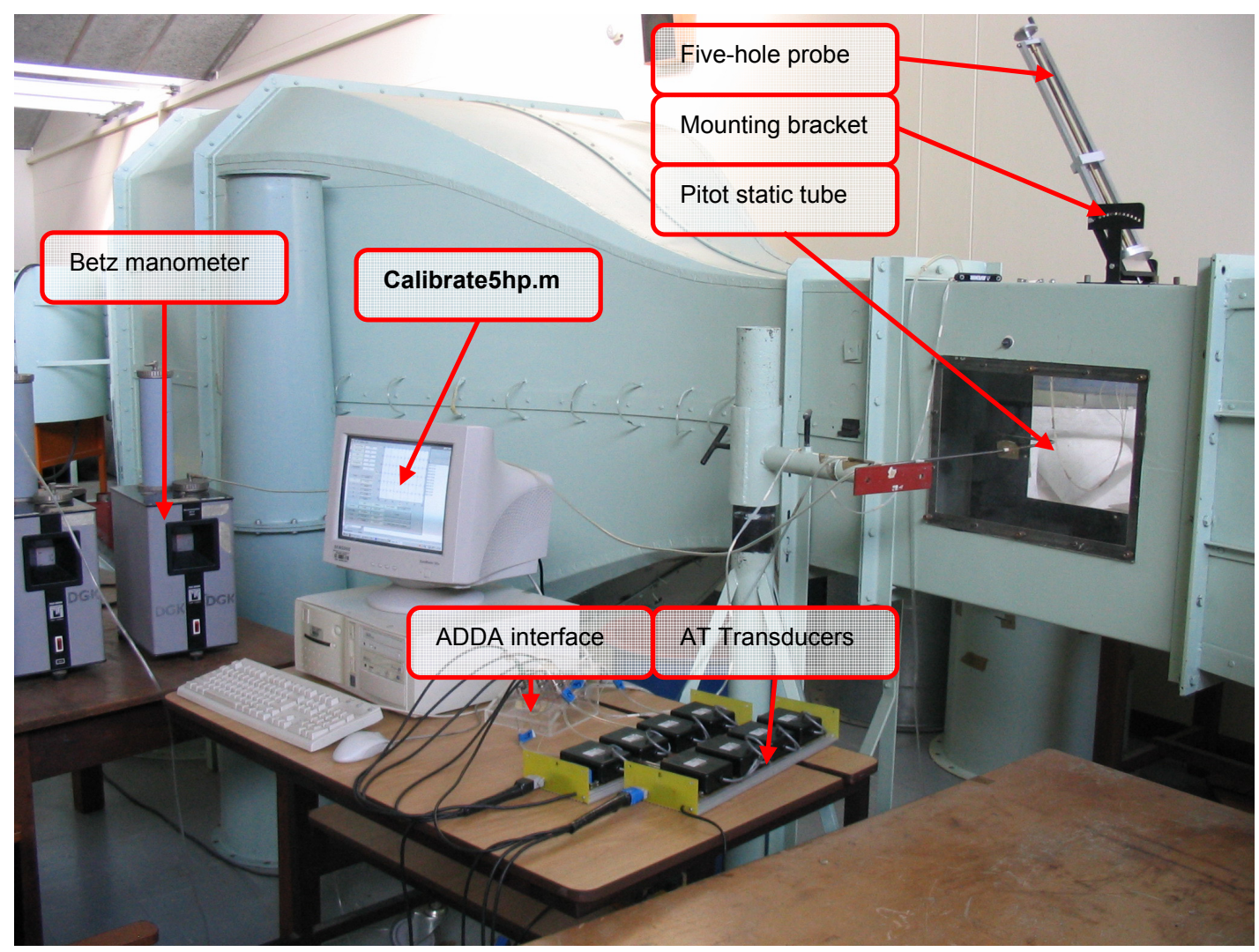

Figure 2-14 - Five-hole probe calibration setup 


\subsubsection{PROCEDURE}

The small wind tunnel is started up with the louvers fully open. To regulate the total velocity in the test section the 'bleeding' gates before the test section are symmetrically opened until the average operating velocity in the experimental rig for this project is obtained, which is approximately $16 \mathrm{~m} . \mathrm{s}^{-1}$. At the time of the calibration the throttling gate of Milandri (2003) was used, instead of the bleeding gates.

The program used to sample the values is calibrate5hp.m. This program samples the coordinates, pressures, and Pitot tube pressure readings, and calculates the probe coefficients and stores them in a 3D matrix. The pressures were converted to pressure coefficients as defined in Strohmaier (1997). The definitions for the coefficients are repeated in $\S 4.2 .1$ for convenience.

The pressure coefficients are then calculated for a range of yaw- and pitch angles. As mentioned, the probe was calibrated with $5^{\circ}$ increments, and the extremities of the angles are $\pm 30^{\circ}$. To save time only about 10 samples per co-ordinate were sampled, which is strictly speaking not enough to know the variance accurately. Walpole et al. (1998) recommends that at least 30 samples should be taken. However for the purpose of this thesis, the precise values of the variance is not important, but rather their order of magnitude.

Once a new map is generated or the old map modified, the results file is copied to the directory containing the sampling program for it to be implemented into the program. 


\subsection{Outer Wall Pressure}

\subsubsection{OBJECTIVES}

De Jager (2000), Gannon (2001), Kirstein (2001) and Lebas (2001) did not resolve the problem of the flow behaviour noted experimentally. Therefore the outer wall experiments that they did were repeated for all the configurations to be compared with the CFX results.

Previous attempts of Gannon, De Jager and Kirstein were compared to only a few points determined experimentally. These points did not take wake effects into account; therefore Lebas modified the SCPP scale model to have many more points at various azimuthal angles along the outer wall of the transition section.

To verify the CFX code in predicting flow behaviour, these results would be useful, since the five-hole probe cannot get results close enough to the walls. This section discusses the experimental procedure and $\$ 4.3$ the results obtained.

\subsubsection{APPARATUS AND EQUIPMENT}

For the experiment the following readings are required:

- The static pressures on the walls of the transition section

- The mass flow rate

To determine each of these the appropriate equipment is required. The instruments used are:

- Master- and slave switch box

- Pressure transducers

- Computer with ADDA card

- Windmill anemometer 


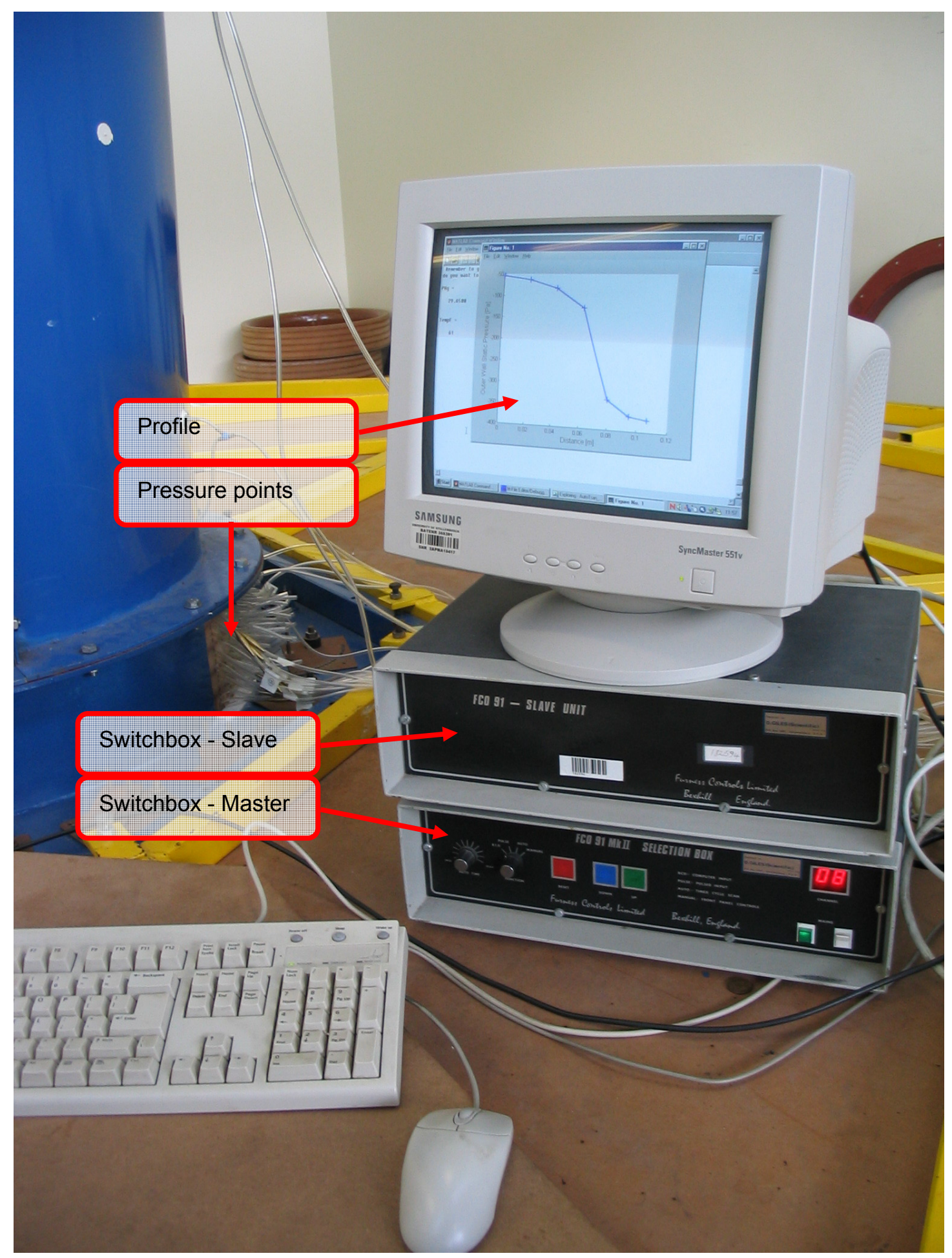

Figure 2-15 - Outer wall experimental setup 


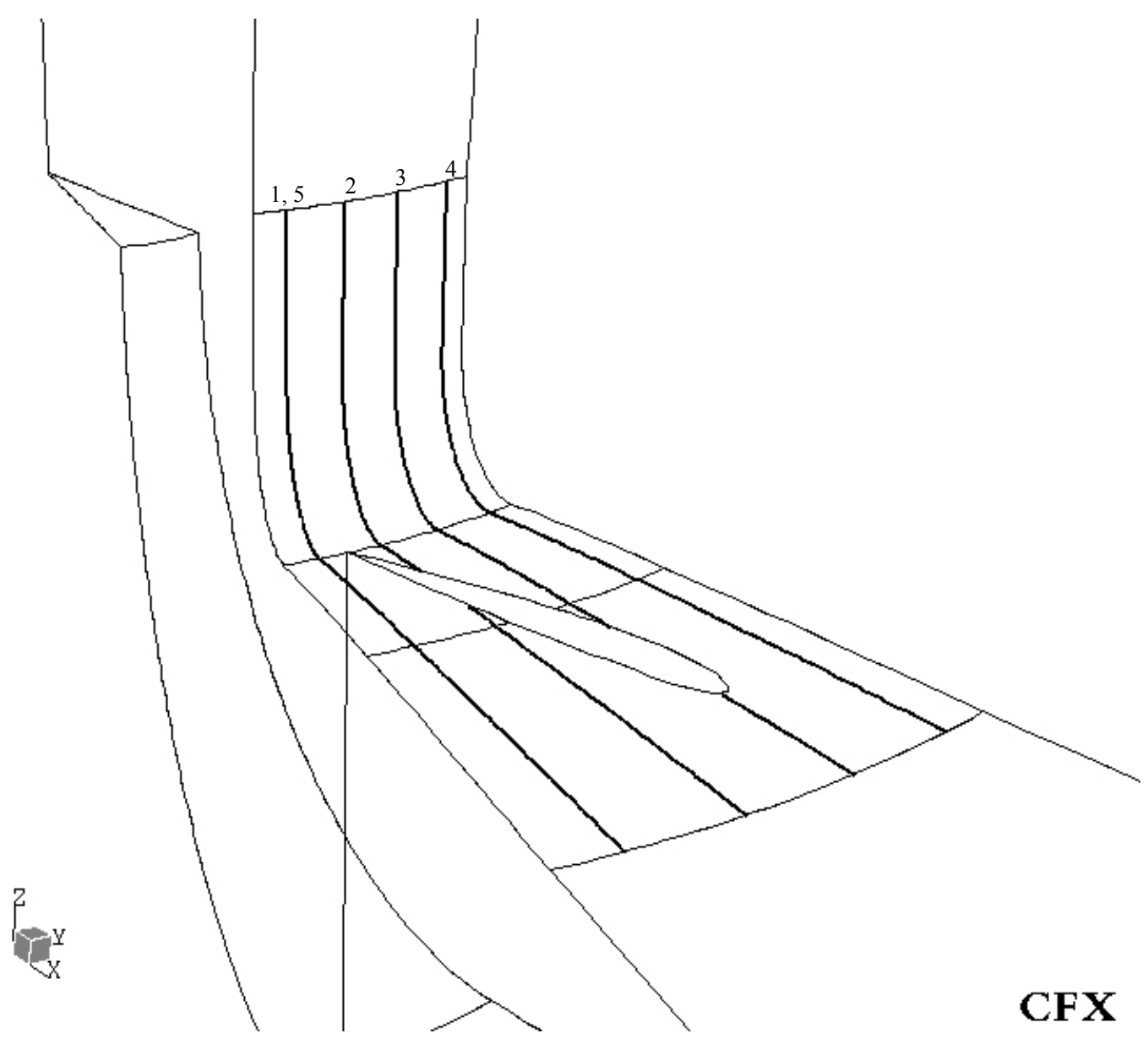

Figure 2-16 - Row location of static pressure tapping points

\subsubsection{PROCEDURE}

The results are gathered by plugging the switch box channels into the appropriate pressure tapping points on the row that is to be sampled on the rig. The switch boxes are set to $\mathrm{BCD}$ for the program to control the switch boxes. The master switch box is plugged into the ADDA card interface and the slave switch box to the master. Each of the switch boxes requires a pressure transducer to the output.

The location for each tapping point is detailed in the sampling software. The first 20 channels are for the outer wall. Channels 21-25 are for the diffuser and the rest for the inner wall. 


\subsection{Five-Hole Probe Traverse}

\subsubsection{OBJECTIVES}

The numerical flow software determines the flow characteristics on all the node points within the flow passage of the transition section and not just on the walls. This experiment enables comprehensive evaluation of the numerical results by accurately measuring the flow characteristics within the flow passage of the transition section so that the results could be compared.

The experimentation generates a grid in the transition section by juxtaposing traverses of the five-hole probe through an orthogonal array of 22 holes. The outer wall experiments are used to validate the numerical package on the wall by determining the wall-static pressure. This experiment however, determines the static- and total pressure and flow direction within the passage of the transition section.

The results of this experiment is important to determine flow separation occurrences, mass-flow, turning angles, average pressure losses and confidence in the numerical packages. This section discusses the experimental procedure and $\S 4.4$ the results obtained.

\subsubsection{APPARATUS AND EQUIPMENT}

The experiment is time-consuming although requiring few components to gather the information. The apparatus is listed below and a few more comments are made from there on.

- Five-hole probe

- Pressure transducers

- Bracket for 5 hole probe positioning

- ADDA card with PC

- Windmill anemometer 
The positions of the profiles were chosen in the meridional plane to be as equally spaced and as square with the flow direction as would be feasible. Figure 2-17 illustrating these positions schematically is shown below.

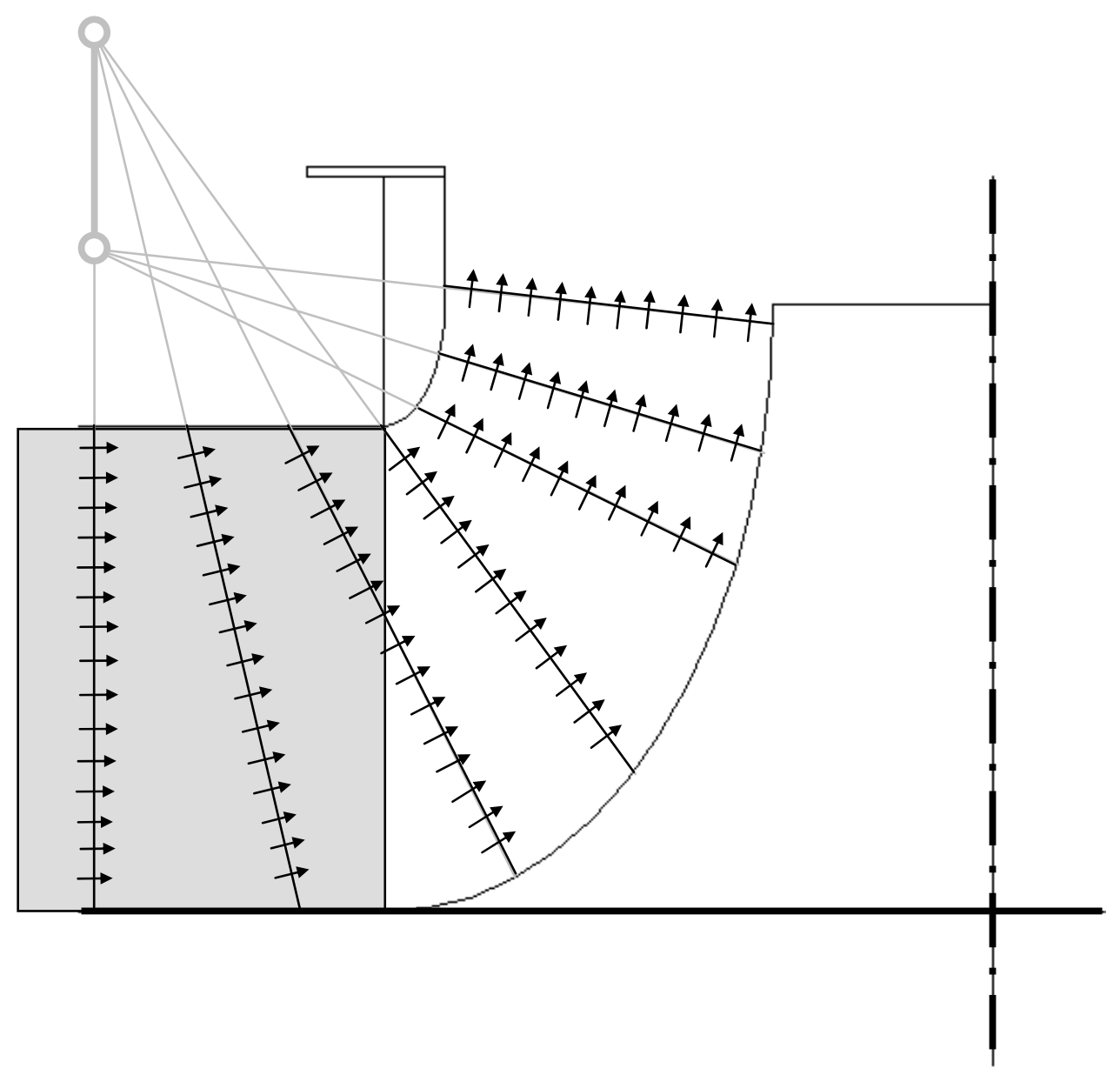

Figure 2-17 - Probe traverse schematic

These traverses originate from the two points shown in the figure above. The bracket holding the five-hole probe in position for the traverses has a slit allowing various origins. However for the purpose of repeatability, only the two extremities of the slit were used. Figure 2-17 shows that this gives a good distribution in the meridional plane.

The next step was to order the traverses in the azimuthal planes. The lower four traverses could not be made in various tangential positions due to interference from the IGVs (especially when the IGV angle is adjusted). Henceforth only the top three planes were expanded azimuthally. 
A special bracket to emulate the five-hole probe action was prepared to drill the holes for the traverses. In order for the drill to be able to move however, the rails on the bracket had a tolerance $(0.5 \mathrm{~mm})$. This means that at the tip of the drill the position could be as far off as $3 \mathrm{~mm}$ when it starts and $6 \mathrm{~mm}$ by the time it has drilled through the wall due to the wood guiding the drill bit. The student attempted to keep the holes as uniform as possible. The top- and middle planes are vertically very well spaced, having a maximum difference of about $1 \mathrm{~mm}$. The lower plane however, due to the initial angle the drill bit meets the wood has a maximum meridional difference of 3 $\mathrm{mm}$. For the two top planes, the difference is insignificant, but not so on the bottom plane.

The bottom plane on the outer wall is $18 \mathrm{~mm}$ from the IGV tail tip. According to the wall pressure measurements, the peak of the pressure gradient is approximately at 15 mm. This means that the mentioned $3 \mathrm{~mm}$ meridional deviation could cause substantial variations in the results. To control these deviations the two outer holes on each row were chosen as the datum whereby to arrange the other holes. If the other holes deviated from their desired locations, they were drilled larger.

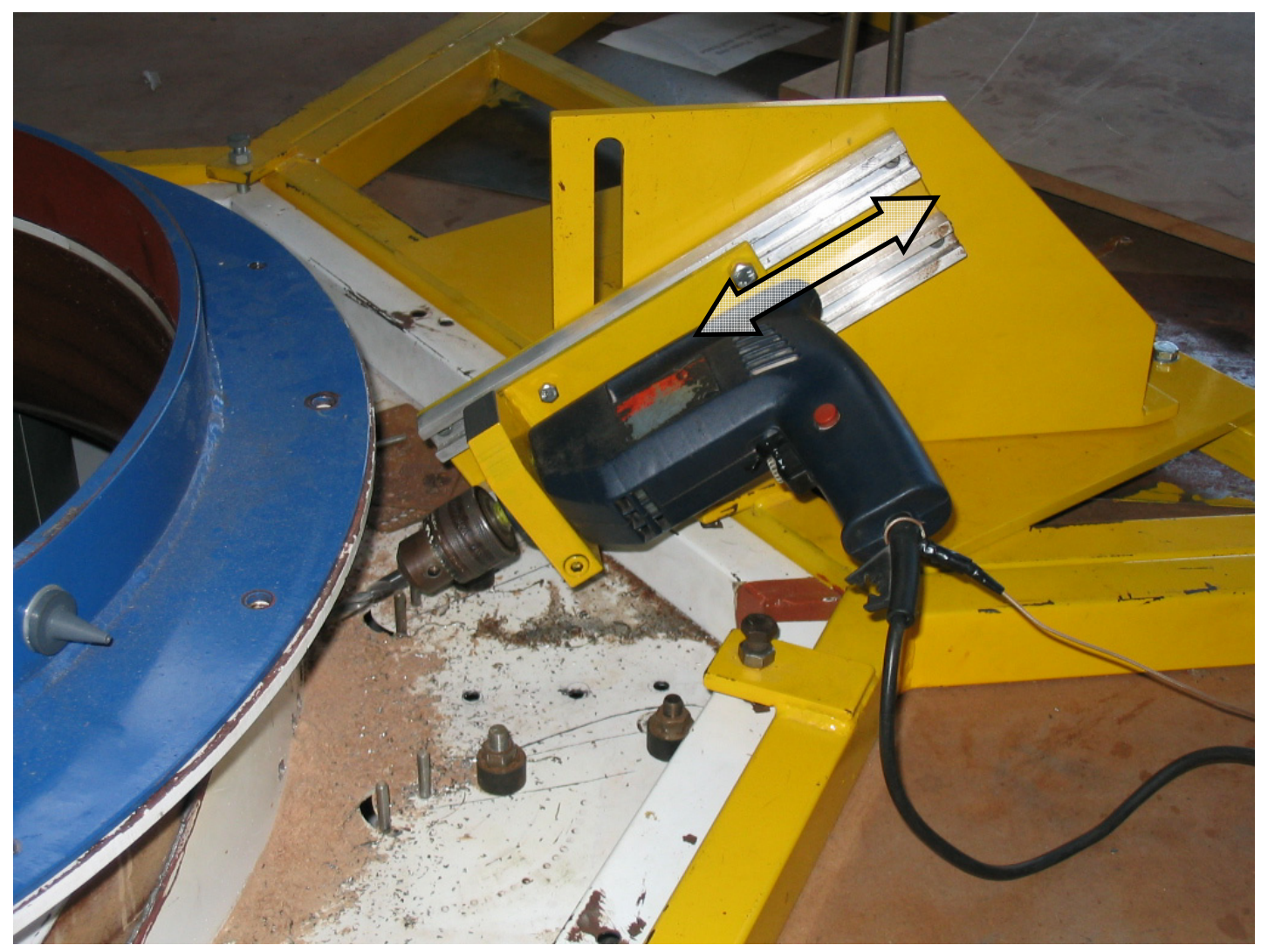

Figure 2-18 - Drill bracket assembly 
To ensure that the positioning of the five-hole probe would be repeatable, markers were drawn on the inner wall, and a Perspex window was located in the diffuser. Figure 2-19 indicates how the angles and orientation in the azimuthal planes were determined. The thin thread lines are highlighted to increase clarity. The azimuthal orientation is estimated to be measured accurately to within $1^{\circ}$ in all the cases, which is acceptable. A digital protractor indicated that the bracket moving azimuthally was level within $0.1^{\circ}$ at all positions.

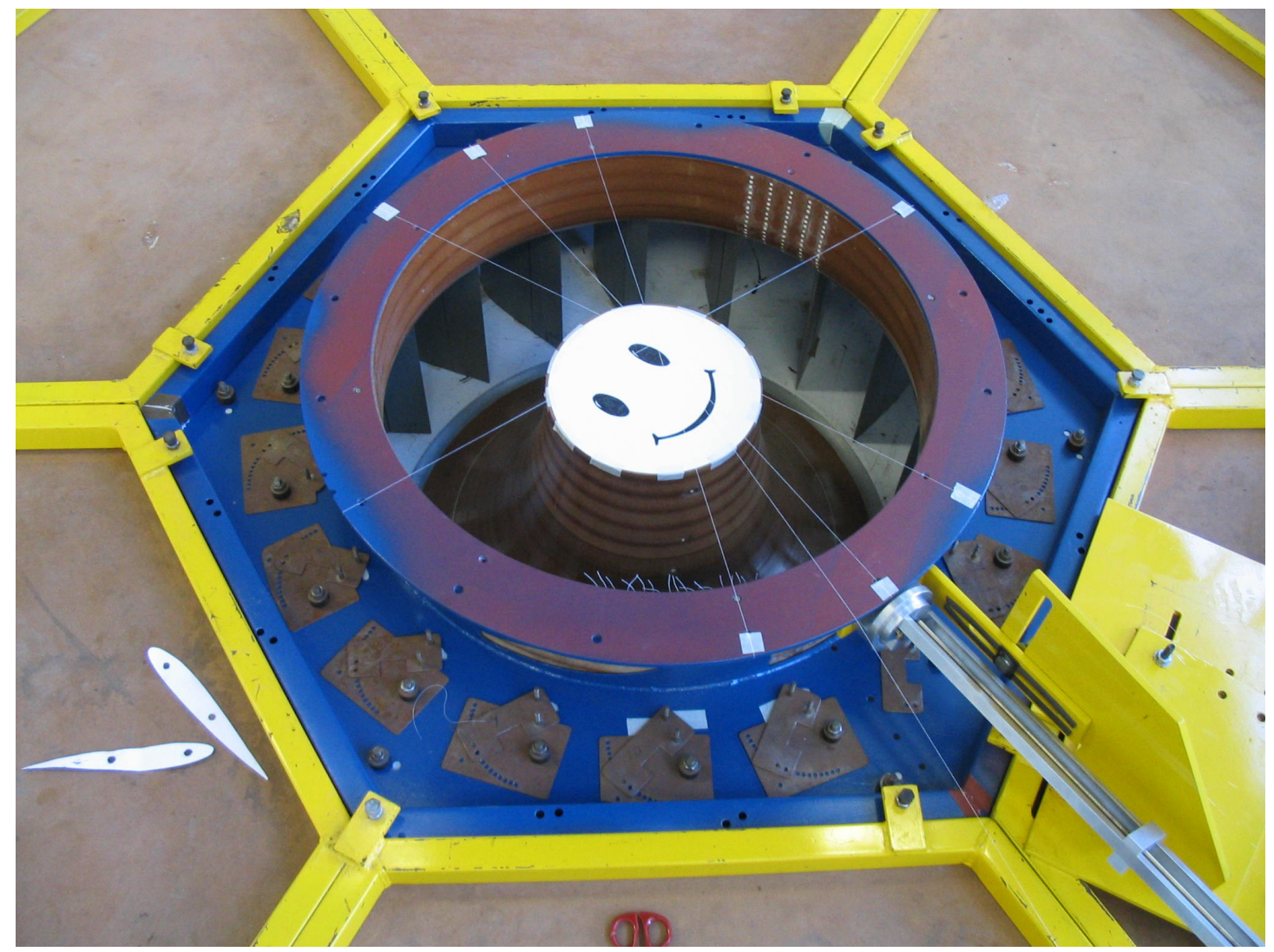

Figure 2-19 - Tangential position verification

The holes for the traverses cause wall suction and henceforth had to be sealed. Figure 2-21 shows sealing method on the inner part of the outer wall. Figure 2-20 shows the neoprene seals used on the outside. The neoprene seals tightly around the sleeve of the five-hole probe and only leaves a small hole on the unused holes (average of 2 $\mathrm{mm}$ diameter). These small-hole leakages were then completely blocked off by taping the hole shut through the Perspex window on the inside.

To reduce the effect of the holes when the probe traverse gets near the outer wall, the small stem was covered as indicated in Figure 2-21. This is supposed to act as a 
pseudo wall; however it was found that this did not affect the results significantly near the walls unless the hole had a much larger diameter than the sleeve.

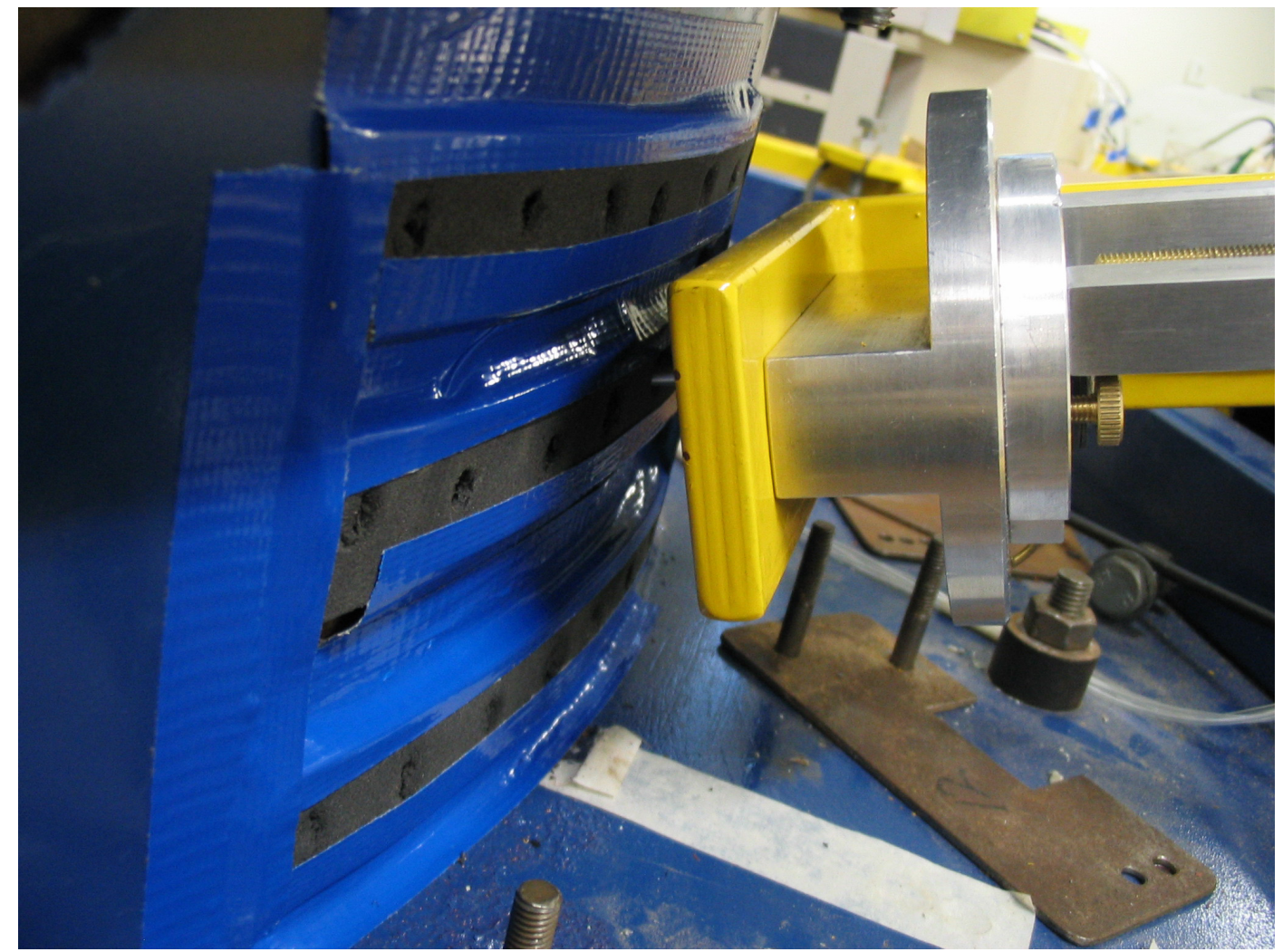

Figure 2-20 - Outer wall hole seals

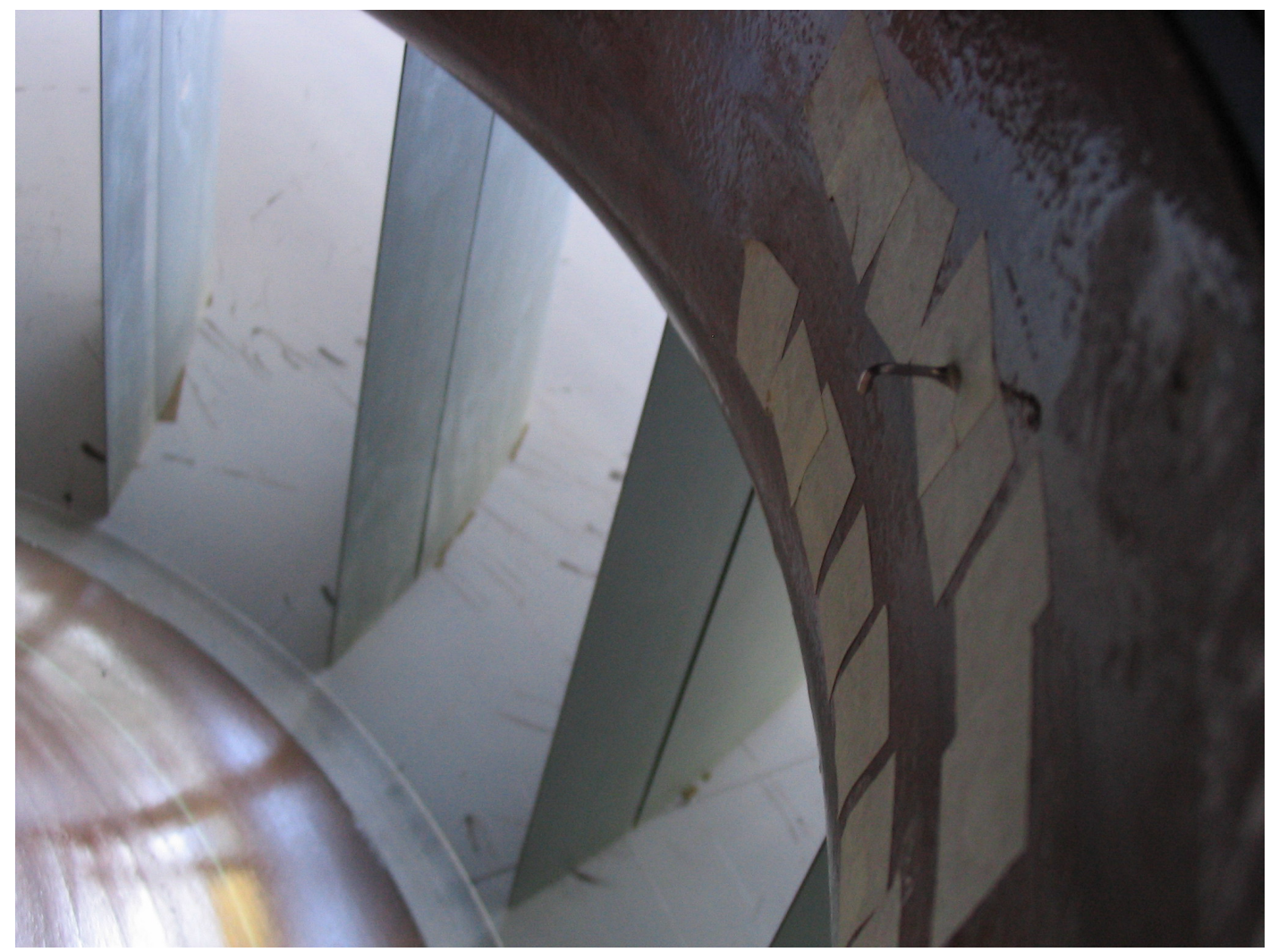

Figure 2-21 - Probe detail near wall 


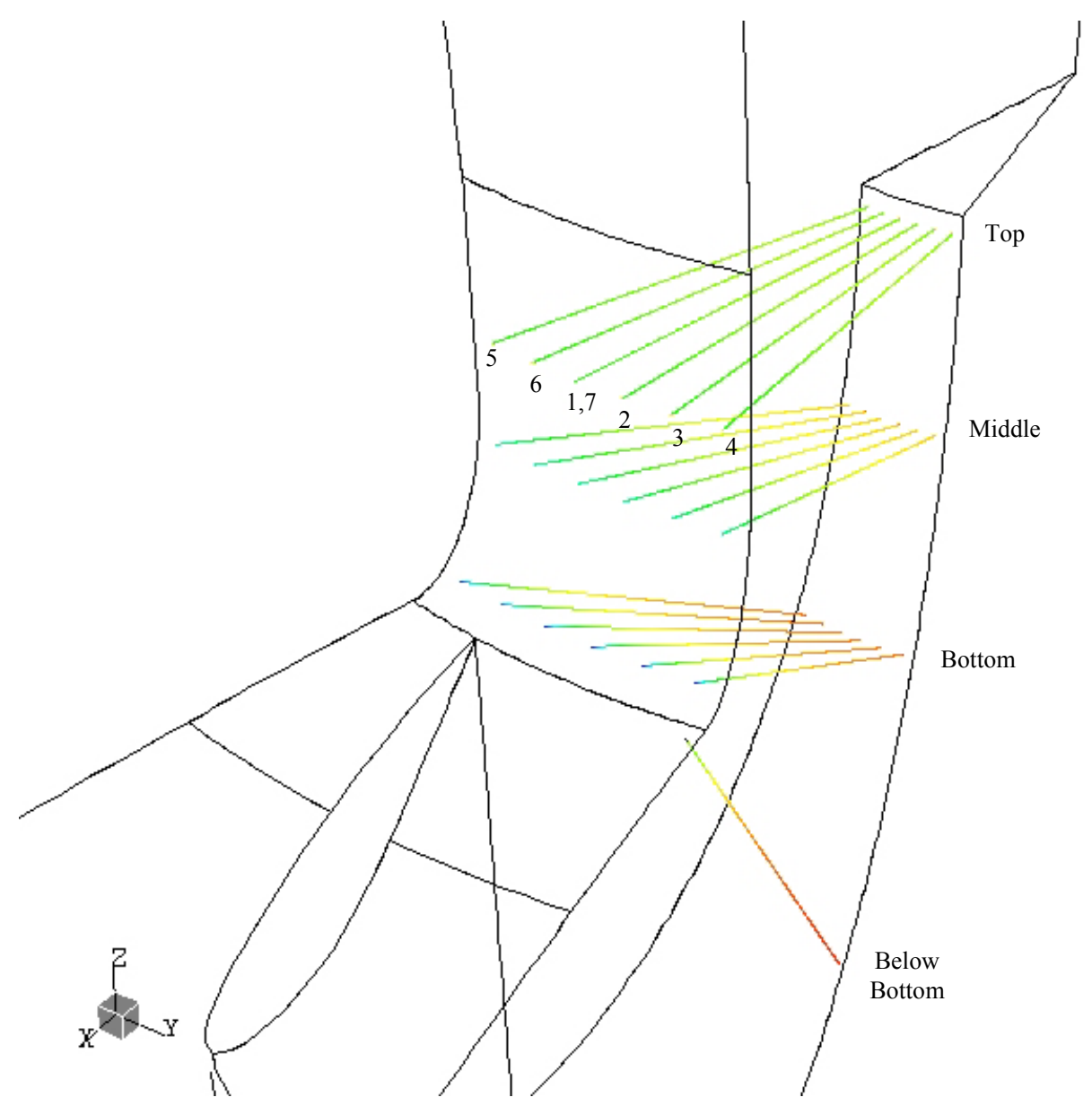

CFX

Figure 2-22 - Traverse line positions for the five-hole probe

Figure 2-22 shows the traverse lines eventually used. The lowest three positions of Figure 2-17 were not included, since the velocities in these regions were too low to be measured accurately with the five-hole probe.

\subsubsection{PROCEDURE}

The five-hole probe was tested and evaluated in the small wind tunnel (§2.3), but to identify the expected behaviour of the flow in advance, a simple visualization check was performed. The test used a rod with a tapered tip with a $40 \mathrm{~mm}$ long white thread attached to its apex. This test is shown in Figure 2-23 with the thread highlighted only slightly. The stability of the thread tip indicates the expected level of turbulence (and hence variance) and possible flow separation near the wall that the probe could be measuring erroneously. The thread remained stable throughout the entire flow regime, except on the top of the hub. This was expected as the flow clearly does break away from the top of the inner cone. 


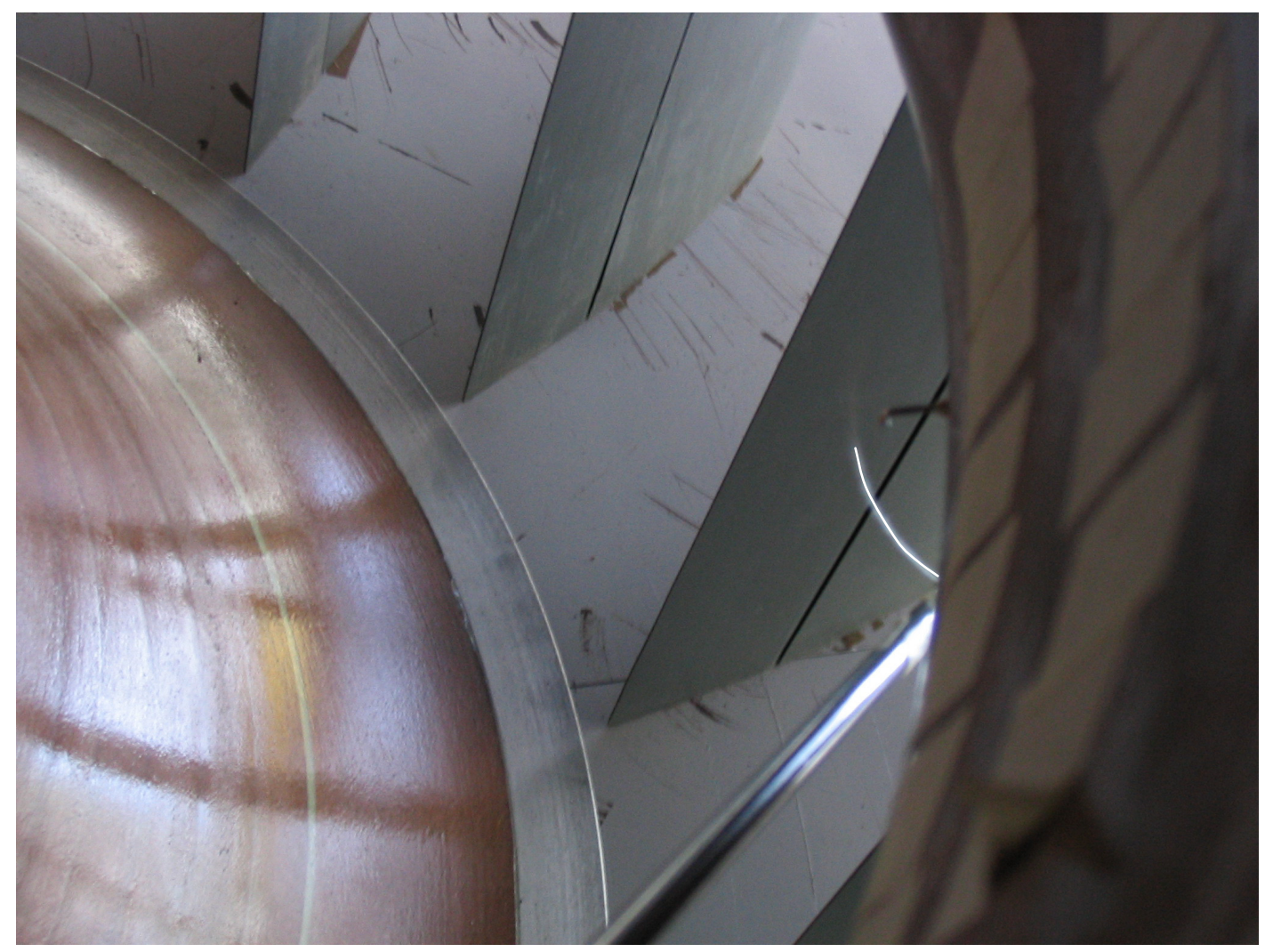

Figure 2-23 - Probe location and flow direction

The probe was mounted in the rig-bracket and prepared to be inserted into the hole. Preparation is done by adjusting the probe to face upward and extending it at least 60 $\mathrm{mm}$ clear from the probe-bracket. The probe is then slid in by hand resting on the bottom of the hole, and then secured in its position with a large wing nut. The wing nut and washers grip into rubber linings and the probe is therefore insensitive to vibration that could cause it to change its position.

Neoprene has a tendency for small particles to break off from it when it is subjected to friction. This is hazardous for the five-hole probe that easily gets blocked. Therefore a sleeve was required on the probe head before insertion (or extraction) for each traversing hole. The rubber sleeve also acted as a shock absorber and protected the probe from frictional damage. Once the probe was in the flow passage and secure, the sleeve was removed through the Perspex window.

This experiment did not use a varispeed drive to reduce the speed of the suction fan. The fan was directly powered and ran at $50 \mathrm{~Hz}$ for all the experiments. 


\section{CHAPTER 3: COMPUTATIONAL FLUID DYNAMICS}

\subsection{Introduction to NuMERICAL Modelling OF THE SCPP}

Using a numerical model to simulate the performance of a design, and especially of the magnitude of the SCPP, is more cost effective than building prototypes or experimental models. However, to an even greater degree than the experimental models, the numerical models are simplified representatives of the real thing (Blanchard and Fabrycky, 1998).

The numerical model of this project focuses only on the internal fluid flow, and more specifically on the internal fluid flow of the collector-to-chimney transition section. Little or no attention has been given to the influences of the other influencing factors named above. The other factors are normalised so that the dependent variables can be investigated by changing the independent variables.

Testing and validation of the numerical software was done prior to any comparisons to the experimental data. However for the sake of brevity these validation cases are omitted from this thesis. These validations did however help to optimise the numerical model from the simplest possible working model to a more complex and complete model. This bottom-up approach to obtain the results served to determine where the problem areas of the transition section are located.

The EFD equipment and procedure were explained in the previous chapter. This chapter describes the procedure to obtain the CFD results. 


\subsection{SOLID MODELLING OF THE SCPP}

\subsubsection{BUILDING THE SOLID MODEL WITH CAD}

The geometry of the transition section is too demanding and complex to build in the CFD package, since elliptic axisymmetric surfaces and smooth curves for the NACA shape of the IGVs were required. For this reason the solid model was built in a separate CAD package, namely Solid Edge v14 ${ }^{\mathrm{TM}}$, and exported to CFX 5.5.1 as an IGES file.

The geometry, as described by Gannon (2002), was rebuilt in Solid Edge and exported in the IGES file format. However, there was difficulty in getting the CFX 5.5.1 to interpret the IGES file correctly due to the large combination of settings in an IGES file. Initially Solid Edge v11 $11^{\mathrm{TM}}$ was used, but none of the combinations used seemed to give a solid that CFX could open. After a period of trial and error it was determined that the CFD software opened the IGES files correctly if the solid model was described in terms of surfaces as it was modelled in Solid Edge v14.

In Solid Edge v14 it is simple to adjust the settings for the solid model so that CFX would open it correctly. When the file is saved as an IGES file, the body type of the solid could be adjusted in the options to: Trim surfaces as NURBS (type 128). If Solid Edge v14 is not available to do the solid modelling, an output file is given in Table 3-1 as an example of how the IGES file should be set up.

Table 3-1 - IGES format details (example)

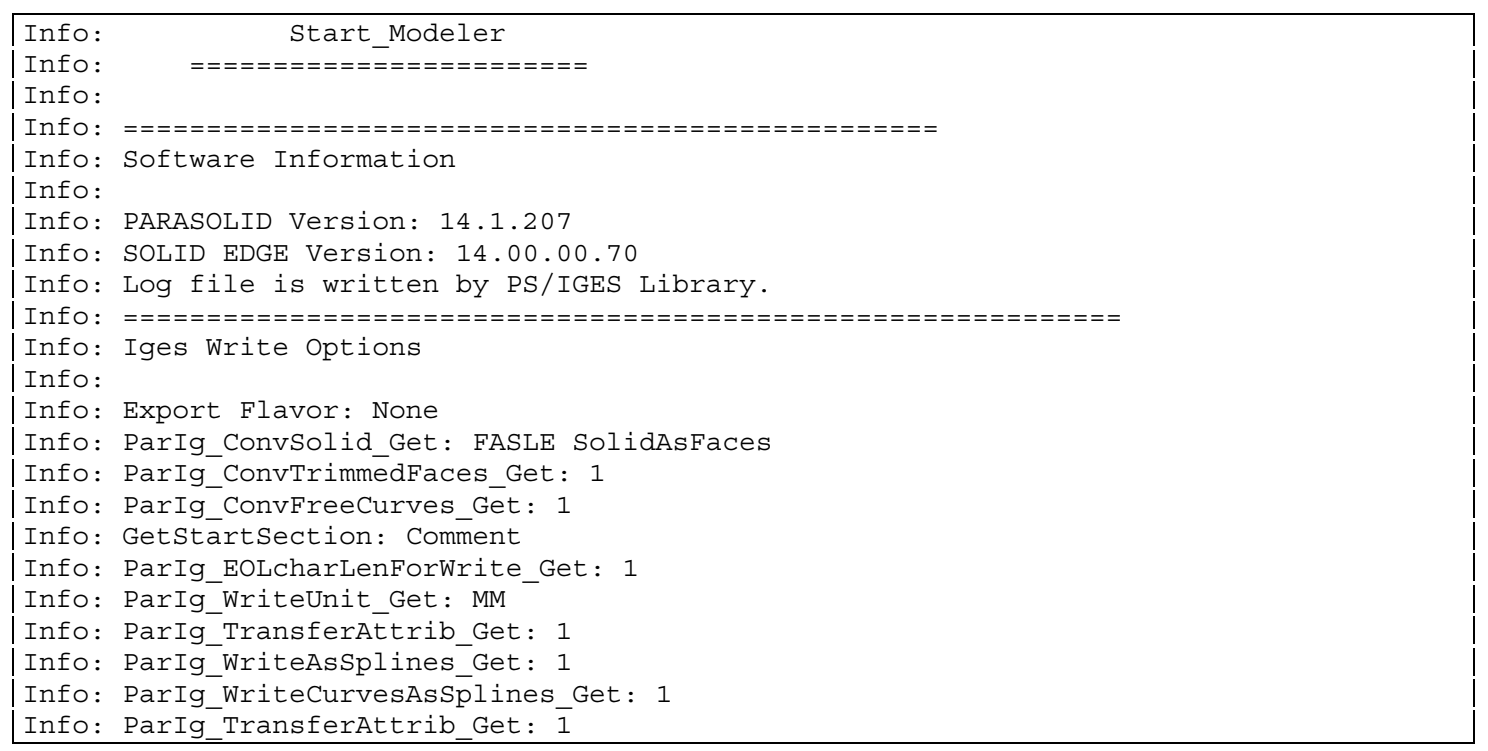




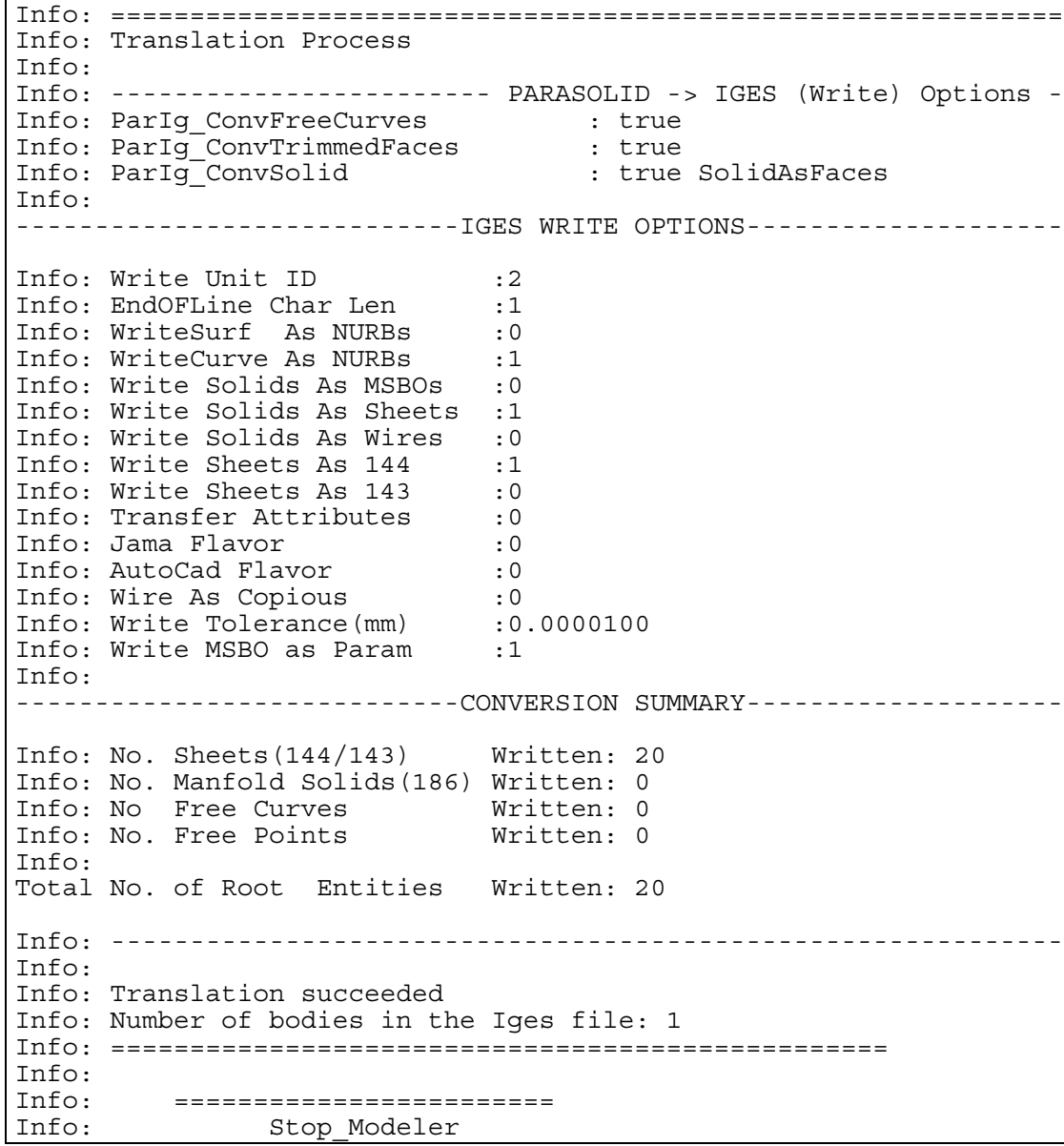

The solid model of the SCPP was built as a negative of the actual model. This means that the flow domain is built as a solid and the structures in the flow domain as gaps. To be able to use the quantity of grid points more efficiently, the axisymmetric property of the SCPP was exploited. An $18^{\text {th }}$ sector of the transition section was built and simulated as a periodic pair (discussed in §3.3.3). The low solidity of the IGV blade row fortunately allows such a section without complications.

The surfaces on the solid model are divided into various sections to facilitate grid generation. This means that the IGVs, for instance, are made up of 4 surfaces: the leading edge, the suction end and two surfaces for the pressure end. However, for the mesh control, the IGV is defined by two sections, namely: the IGV nose and the rest of the IGV. 


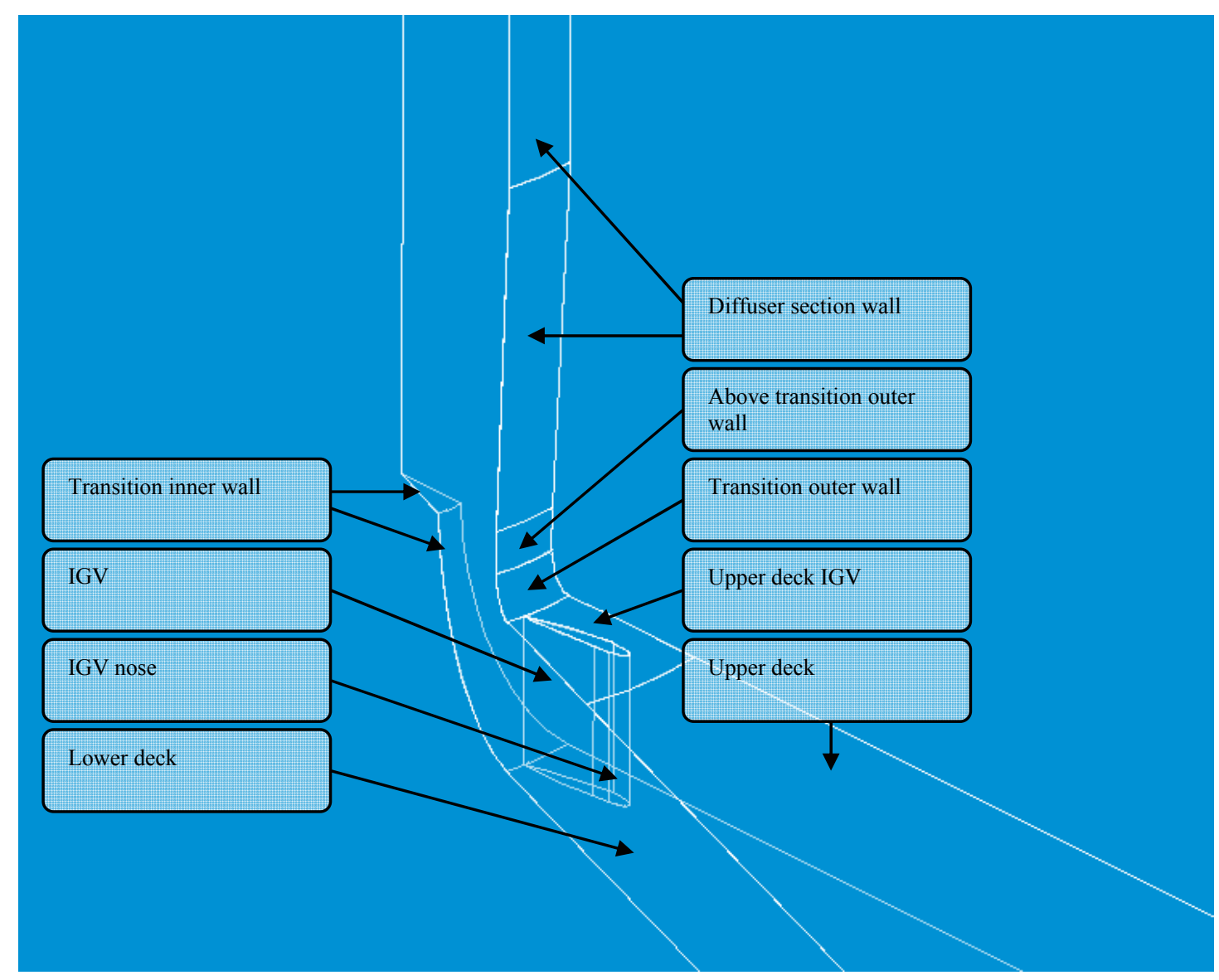

Figure 3-1 - Solid model surface sections

\subsubsection{IMPORTING THE SOLID MODEL TO CFX}

Once the model has been completed and saved in the correct format as an IGES file, CFX can open the solid model. The imported file is interpreted in terms of surfaces, to which CFX allocates surface numbers automatically. It was determined that a global geometric tolerance of 0.001 gave the least problems in importing the surfaces.

Some of the surfaces on the model are fragmented and mismatched, and henceforth are rebuilt in CFX build. The fragmented surfaces are deleted and the new surfaces are created by using the "create/surface/revolve" command from the defined curve shapes. Once the fragmented surfaces are repaired, the "edit/surface/sew" command is used to close the gaps between the mismatched surfaces. Once the mismatched surfaces are joined, all the surfaces are chosen, where after the "create/solid/B-rep" command is used to convert the surfaces to a solid. Once the solid is created the CFD analyses can begin. This procedure was facilitated by the low tolerance values in the IGES file generated by Solid Edge ${ }^{\mathrm{TM}}$ (Table 3-1). 


\subsection{CFD ANALYSIS}

\subsubsection{PRELIMINARY INVESTIGATION OF FLOW}

CFD software is limited because of its inability to predict flow transition. Even if CFX 5.5.1 had the facility to calculate the transition from laminar to turbulent flow, the results would be suspect since reliable engineering techniques for predicting transition are deficient in CFD software (Oberkampf and Blottner, 1998; Crouch et al., 2002).

In this project the Reynolds number of the flow leading up to the transition section in the scale model is so low that the flow is laminar. The Reynolds number beyond the transition section is so high that the flow is turbulent. This is challenging, since it means that flow transition would occur somewhere in between the entrance and exit of the transition section. The calculations of $\S$ A.1 confirms that the flow regime changes through the transition section.

Simulating this transition of flow regime is beyond the scope of this project; therefore the analyses are done keeping in mind that secondary effects due to the transition from laminar- to turbulent flow could occur.

\subsubsection{MeSHING}

The meshing was done using the unstructured meshing technique of CFX 5.5.1. The unstructured mesh is simpler, has a consistent amount of diffusion (\$3.3.4) and is more stable than structured meshes, especially for complex geometries such as the one simulated in this project. One disadvantage of using an unstructured mesh is that more node points are required than with a structured mesh. The reason is when the walls are simulated that the tetrahedral- or hexahedral cells of the unstructured mesh are too coarse to capture the boundary layer adequately. To make the unstructured mesh fine enough is usually prohibitively expensive for accurate simulations over thin bodies.

For optimal grid generation, it is possible to use a combination of structured and unstructured meshing using advanced techniques in PATRAN. It was considered to be unnecessarily complex and time consuming to use such meshing techniques for this 
application. Therefore the meshing technique used throughout the project is the unstructured meshing. The unstructured meshing technique is explained in the rest of this chapter.

CFX generates a surface mesh of triangular elements using either the Delaunay triangulation method or the advancing front (AF) method. The Delaunay method generates the surface mesh faster although the mesh quality is not as good as the AF method. The Delaunay method was used in this project, which is able to generate a mesh on closed surfaces (CFX-5 Help). From the triangular surface mesh elements the volume mesh elements are grown using the advancing front and inflation (AFI) built into CFX. The AFI grows tetrahedral elements (and prismatic and pyramidal elements when inflation is used) from the surface mesh elements (CFX-5 Help).

To create a computationally accurate mesh, the prismatic elements create nodes close and normal to the surfaces when inflation is used. The inflation will be discussed later in this section when looking at the near wall simulation.

\section{Mesh Parameters}

To start the process of meshing, a background length scale is chosen from where the whole mesh is based. The mesh controls override the mesh parameter where it is applicable.

For this project volume spacing was used, that allows the maximum edge length of any element not to exceed a specified value. Normally CFX would choose the maximum edge length as $5 \%$ of the maximum model dimension. This value did not allow enough nodes in the collector region for the boundary layer to develop adequately; therefore the maximum edge length were chosen as $60(\mathrm{~mm})$ for the simulation of the experimental rig.

Neither edge- nor surface- proximity was used as a mesh parameter. It was found that CFX could not generate a mesh with these options enabled. That is probably due to the complexity of the geometry of the IGV. The option for a target number of nodes was also not used. CFX also had problems in generating a mesh consistently with this option enabled. The mesh controls were instead chosen to create the resolution 
desired. Stretching is not a wise option for the simulation of the transition section, since the flow turns almost $90^{\circ}$ both axially and tangentially.

\section{Mesh Controls}

Extensive mesh controls were implemented into the total volume mesh. Since the nature of the flow was already known to a large extent (§2.5.3) as well as the exact positions where the samples were going to be taken experimentally (Figure 2-22 \& Figure 2-16), the grid could be refined in the critical areas to obtain the best results.

Table 3-2 contains the co-ordinates for the traverse mesh controls on the inner and outer walls of the model. Each of these routes was divided into 500 points along the way for use in the results. The mesh was never so fine that 500 points could be used along these routes; therefore the answers are interpolated at these points. For the mesh control the two extreme co-ordinates of the route were required. Each of these controls were given a maximum edge length of $5(\mathrm{~mm})$, a geometric expansion factor of 1.1 and a radius of 0 . The effect of the mesh refinement is indicated in Figure 3-2.

Table 3-2 - Traverse co-ordinates in CFX; 260mm deck height

\begin{tabular}{|c|c|c|c|c|c|c|c|c|}
\hline \multirow{2}{*}{\multicolumn{2}{|c|}{ Row }} & \multirow[b]{2}{*}{ theta } & \multicolumn{3}{|c|}{ On Inner Wall [mm] } & \multicolumn{3}{|c|}{ On Outer Wall [mm] } \\
\hline & & & $\mathbf{x}$ & $y$ & $\mathbf{z}$ & $\mathbf{x}$ & $y$ & $\mathbf{z}$ \\
\hline \multirow{3}{*}{1} & $\mathrm{~T}$ & \multirow{3}{*}{-1.7463} & 143.9465 & -4.38866 & 330.541 & 359.8298 & -10.9705 & 361.7801 \\
\hline & M & & 152.9583 & -4.66341 & 234.5944 & 361.0979 & -11.0092 & 317.1173 \\
\hline & $B$ & & 180.9162 & -5.5158 & 132.8011 & 384.1871 & -11.7131 & 264.3669 \\
\hline \multirow{3}{*}{2} & $T$ & \multirow{3}{*}{1.58706} & 143.9581 & 3.988577 & 330.541 & 359.8589 & 9.970434 & 361.7801 \\
\hline & $M$ & & 152.9707 & 4.238283 & 234.5944 & 361.1271 & 10.00557 & 317.1173 \\
\hline & $B$ & & 180.9308 & 5.012962 & 132.8011 & 384.2181 & 10.64534 & 264.3669 \\
\hline \multirow{3}{*}{3} & $T$ & \multirow{3}{*}{4.93039} & 143.4805 & 12.37728 & 330.541 & 358.665 & 30.94008 & 361.7801 \\
\hline & $M$ & & 152.4631 & 13.15217 & 234.5944 & 359.929 & 31.04912 & 317.1173 \\
\hline & $B$ & & 180.3305 & 15.55614 & 132.8011 & 382.9434 & 33.03445 & 264.3669 \\
\hline \multirow{4}{*}{4} & $T$ & \multirow{3}{*}{8.2537} & 142.5217 & 20.67406 & 330.541 & 356.2682 & 51.67992 & 361.7801 \\
\hline & M & & 151.4443 & 21.96836 & 234.5944 & 357.5238 & 51.86205 & 317.1173 \\
\hline & $\mathrm{B}$ & & 179.1255 & 25.98377 & 132.8011 & 380.3844 & 55.17819 & 264.3669 \\
\hline & $\mathrm{BB}$ & 9.5 & 253.5674 & 42.43263 & 8.086535 & 404.3771 & 67.66952 & 260 \\
\hline \multirow{3}{*}{5} & $T$ & \multirow{3}{*}{-8.4129} & 142.4637 & -21.07 & 330.541 & 356.1232 & -52.6696 & 361.7801 \\
\hline & $M$ & & 151.3827 & -22.3891 & 234.5944 & 357.3783 & -52.8553 & 317.1173 \\
\hline & $B$ & & 179.0526 & -26.4814 & 132.8011 & 380.2296 & -56.2349 & 264.3669 \\
\hline \multirow{3}{*}{6} & $\bar{T}$ & \multirow{3}{*}{-5.0796} & 143.4478 & -12.7509 & 330.541 & 358.5832 & -31.874 & 361.7801 \\
\hline & \multirow{2}{*}{$\begin{array}{l}M \\
B\end{array}$} & & 152.4284 & -13.5492 & 234.5944 & 359.8469 & -31.9863 & 317.1173 \\
\hline & & & 180.2894 & -16.0257 & 132.8011 & 382.8561 & -34.0316 & 264.3669 \\
\hline & & & & & $\begin{array}{l}\text { 6T OW to } \\
\text { IGV tip }\end{array}$ & 83.586 & $\mathrm{~mm}$ & \\
\hline & & & & & $\begin{array}{c}\text { Actual } \\
\text { value }\end{array}$ & 85 & $\mathrm{~mm}$ & Acceptable \\
\hline
\end{tabular}


To obtain the co-ordinates for the $320 \mathrm{~mm}$ deck height configuration, add 60 (mm) to the $\mathrm{z}$-values in the table above.

Also indicated in Figure 3-2 is the mesh control for the top of the trailing edge of the IGV. It was found that this area in particular is very sensitive to the mesh constructed. The best results were obtained by creating a point control with a maximum edge length of $5(\mathrm{~mm})$, a radius of $100(\mathrm{~mm})$ and a geometric expansion factor of 1.2. This is also indicated in Figure 3-2.

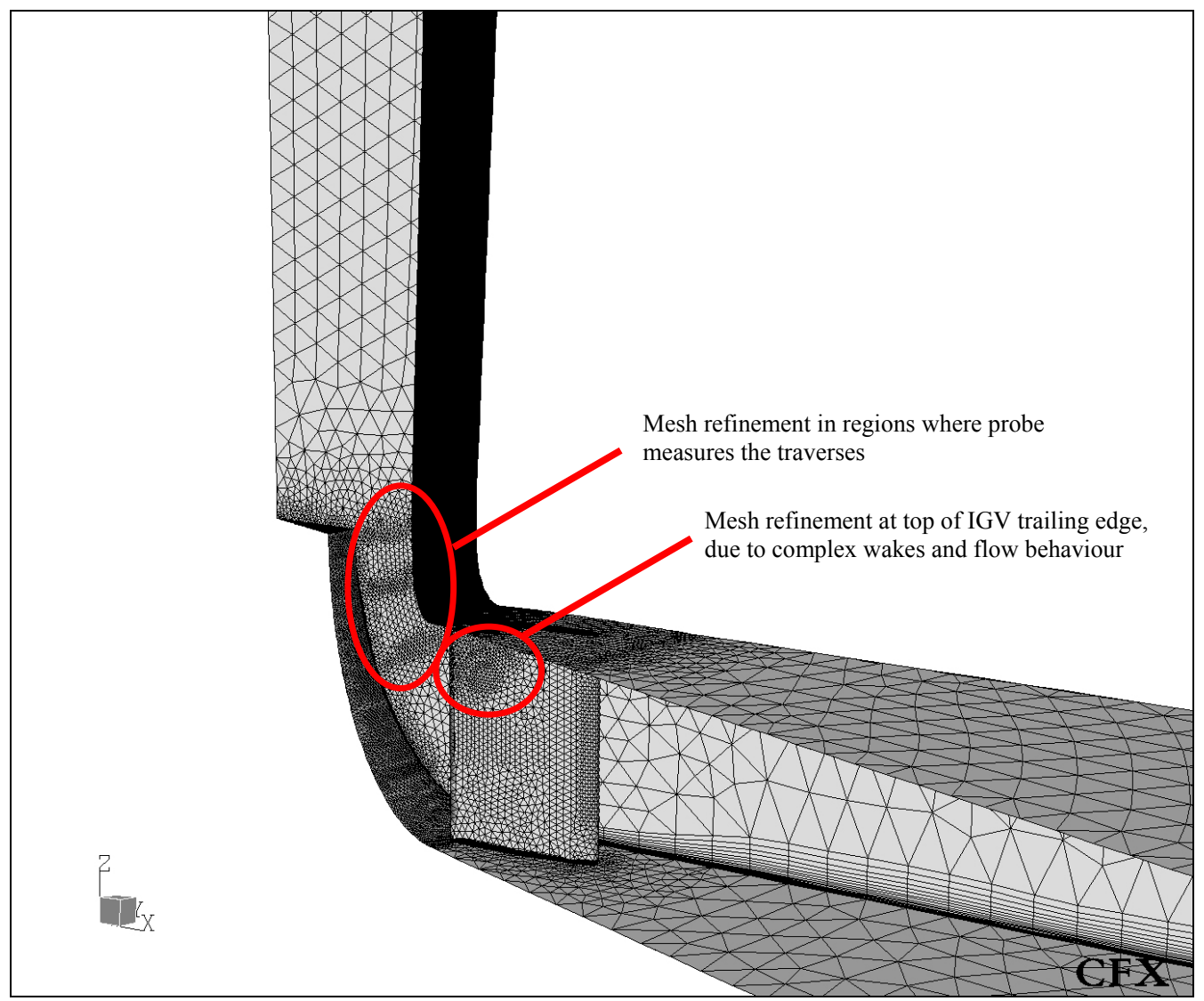

Figure 3-2 - Surface mesh for a 320mm deck height

Another area where the mesh was refined was on the outer wall on the elliptic toroidal section. This is shown in Figure 3-4. The flow turns from radial- to axial and exhibits complex behaviour that requires a finer mesh. Mesh refinement analyses (a sample shown in Figure 3-3) indicated that for the results on the outer wall, such a fine mesh is not necessary. The fine meshing also makes the $\mathrm{y}^{+}$value lower than suggested by the CFX Help. However due to the numerical problems a short distance away from the wall, the maximum edge length in this area was made $2.5(\mathrm{~mm})$, to help refine the 
mesh away from the wall. The numerical problems are addressed in more detail in $\$ 3.3 .4$ and the meshing near the wall later on in this section.

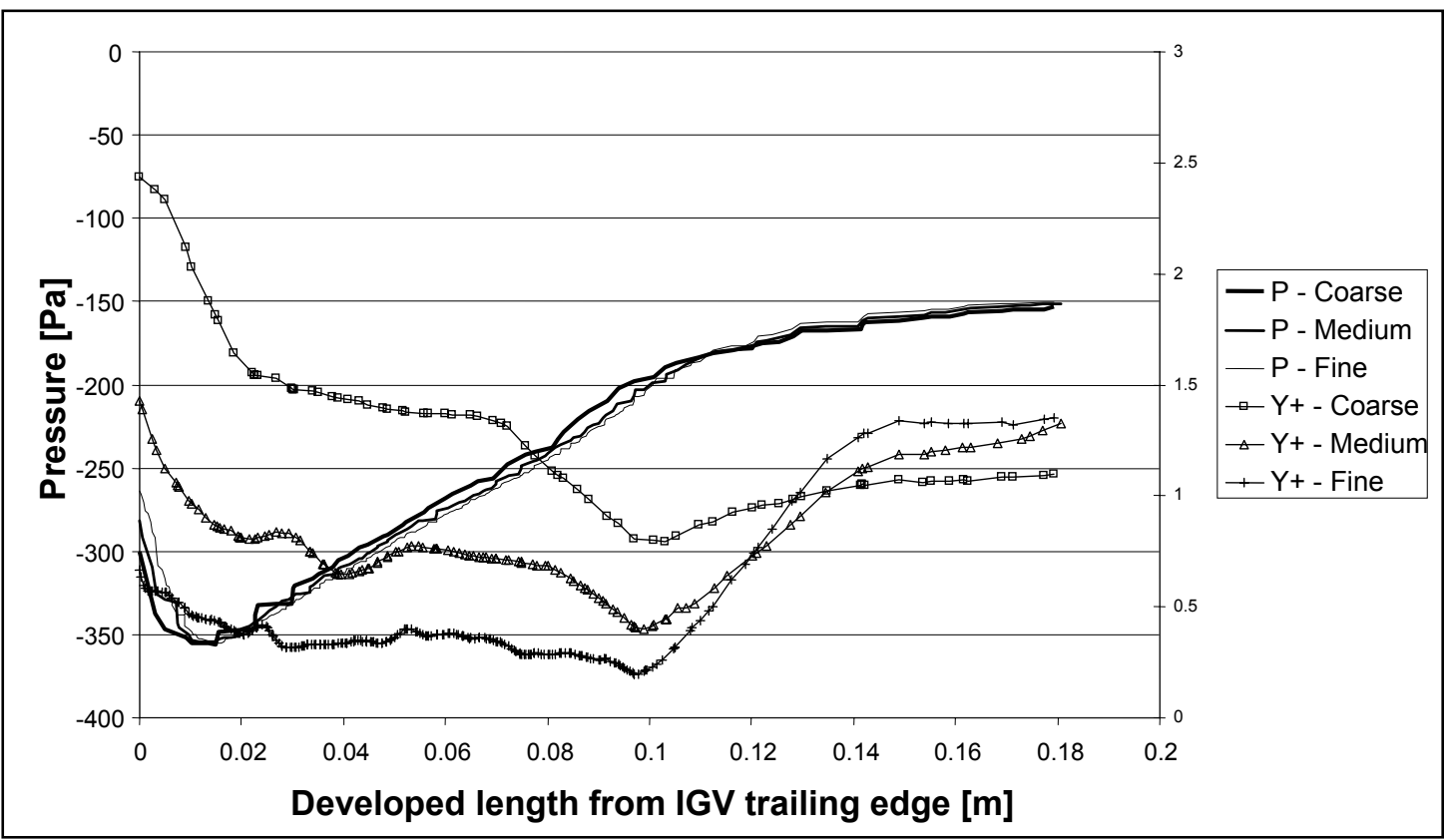

Figure 3-3 - Mesh verification on outer wall; pressure (left axis) and $y^{+}$(right axis)

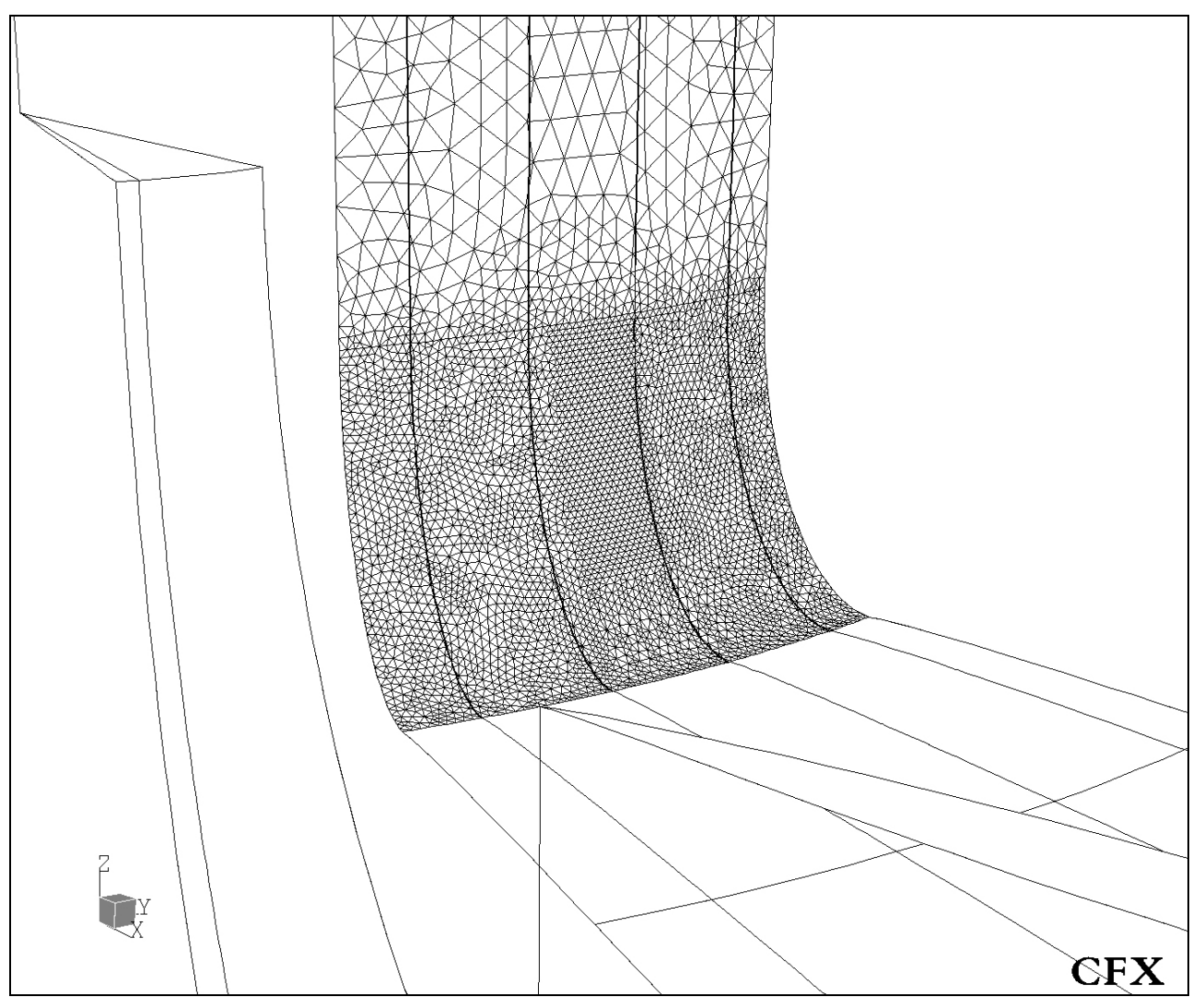

Figure 3-4 - Surface mesh, detail of outer wall 


\section{Modelling Flow Near the Wall}

For this project the wall-function approach of CFX is used which is based on the method of Launder and Spalding (1974). In the wall-function approach, the viscosity affected sublayer region is bridged by employing empirical formulas to provide nearwall boundary conditions for the mean flow and turbulence transport equations. These equations connect the dependent variables at the near-wall grid points to the wall conditions.

The near-wall region can be divided into two layers, namely the viscous sublayer, where viscous effects are dominant, and the fully turbulent layer, where turbulent effects are dominant. Between the two layers there is a region where turbulence and viscosity contributes equally significantly, and this region is also taken into account. These subdivisions are shown in Figure 3-5 below.

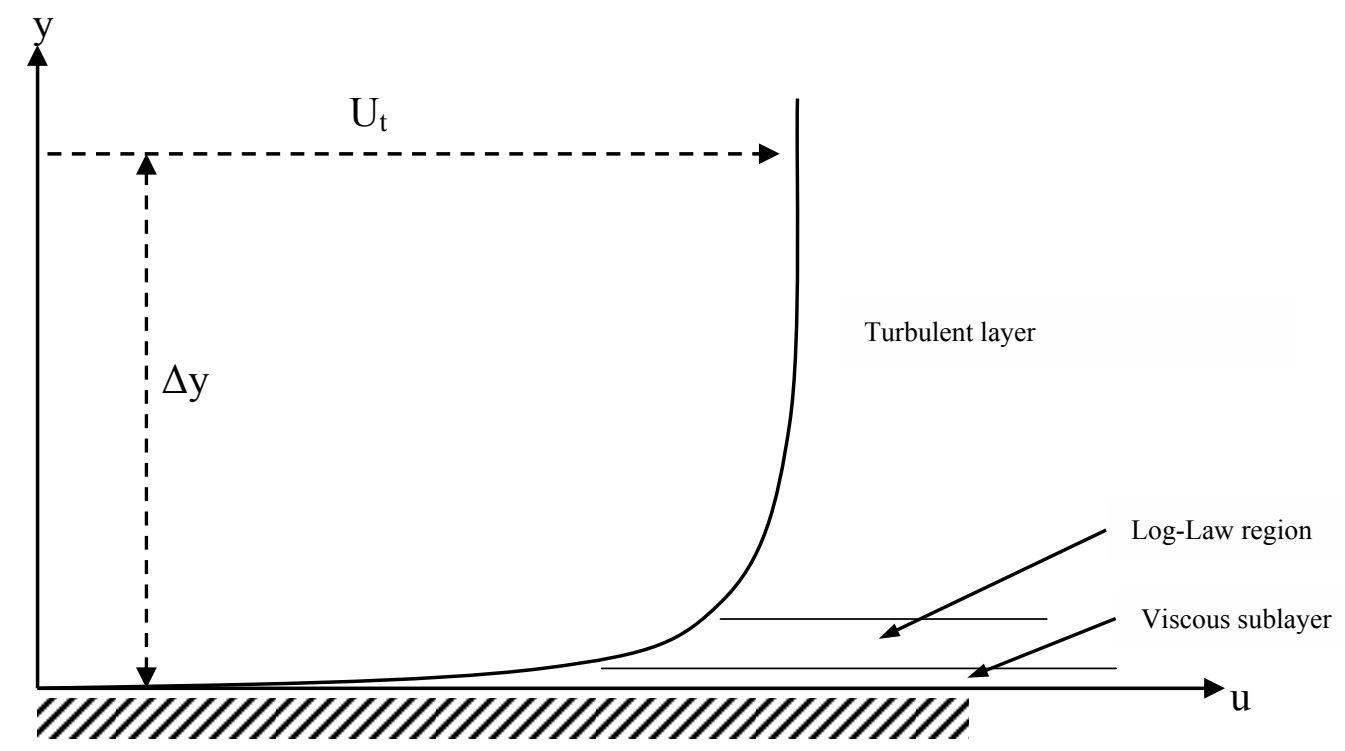

Figure 3-5 - Subdivisions of the near-wall region

CFX 5.5.1 makes use of scalable wall functions for all turbulence models based on the $\varepsilon$-equation. The alternative is to resolve the boundary layer details with a fine enough mesh, but this would have required a prohibitively fine mesh. Thus the advantage of the wall-function is that relatively coarse meshes can be used to do the modelling. The disadvantage is that the predictions depend on the location of the grid point nearest to the wall. This makes the function sensitive to near wall meshing and it means that refining the mesh would not necessarily improve the accuracy (Grotjans and Menter, 1998). However Figure 3-3 suggests that refining the mesh in this case does improve 
the accuracy, and besides this near wall meshing problem is relieved by the scalable wall-function.

The wall function assumes that the surface coincides with the edge of the viscous sublayer, which is defined to be at $\mathrm{y}^{+}=11$. The computed $\mathrm{y}^{+}$value is not supposed to fall below this limit. The aim is to get at least 10 nodes in the Log-Law region, without getting nodes within the viscous sublayer region to avoid fine grid inconsistencies.

The problem is that the grid has to be generated and the flow solved before the $\mathrm{y}^{+}$ values can be determined. Therefore a preliminary study has to be made to be able to estimate the grid spacing. The CFX Help file recommends that a flat plate assumption is made and the grid spacing calculated accordingly. It was however found to be an inadequate assumption for this application and henceforth the adequate grid sizes were determined by trial and error.

Surprisingly the grid configuration that gave the best answers were not from the model with the $\mathrm{y}^{+}$values set up as the CFX Help suggests. When the recommendations of the CFX Help files are followed to the letter, the inflated boundary layers interfere with each other thus preventing CFX from being able to generate a mesh.

Inflated boundary layers are used to get the node points close to the wall, which is usually required for $y^{+}$values of 11 . The inflated boundary layers are extruded prisms from the triangular surface elements. They rapidly increase in length normal to the surface until the free stream edge length is achieved according to a geometric expansion factor. This expansion factor is the multiplier of the previous element length. This means that if the expansion factor is 1.2 , then the length of that element would be 1.2 times that of the one adjacent to it toward the wall. The user chooses how many of these prismatic layers are to be used, and can choose the length of the node closest to the wall. This makes the process of grid generation much more expensive time wise, since the grids' setup has to be iterated. 
The final grid setup's $y^{+}$values in this project are shown in Figure 3-6. By trial and error it was established that this setup gave results that compared best with the experimental ones. For the collector-, outer wall- and the inner wall region, the $\mathrm{y}^{+}$ values are very low being less than 5 . This is not what was suggested by the CFX Help that recommends $\mathrm{y}^{+}>11$. The setup suggested by CFX Help however did not give better answers in this case. The reason may be that the wall functions assume turbulent flow throughout the flow regime, and the flow is predominantly laminar until it has gone a distance around the outer wall. The diffuser region has $\mathrm{y}^{+}$values that tend toward the suggested value of 11 , which makes more sense since the flow is fully turbulent in this region. The IGV could not make use of inflated boundaries, due to numerical errors in the grid generation. The mesh in this region was however generated within the recommended $11<\mathrm{y}^{+}<300$. This simulated the wake from the IGV most accurately in the traverse results (see $§ B .1$ to $\S B .4$ ).

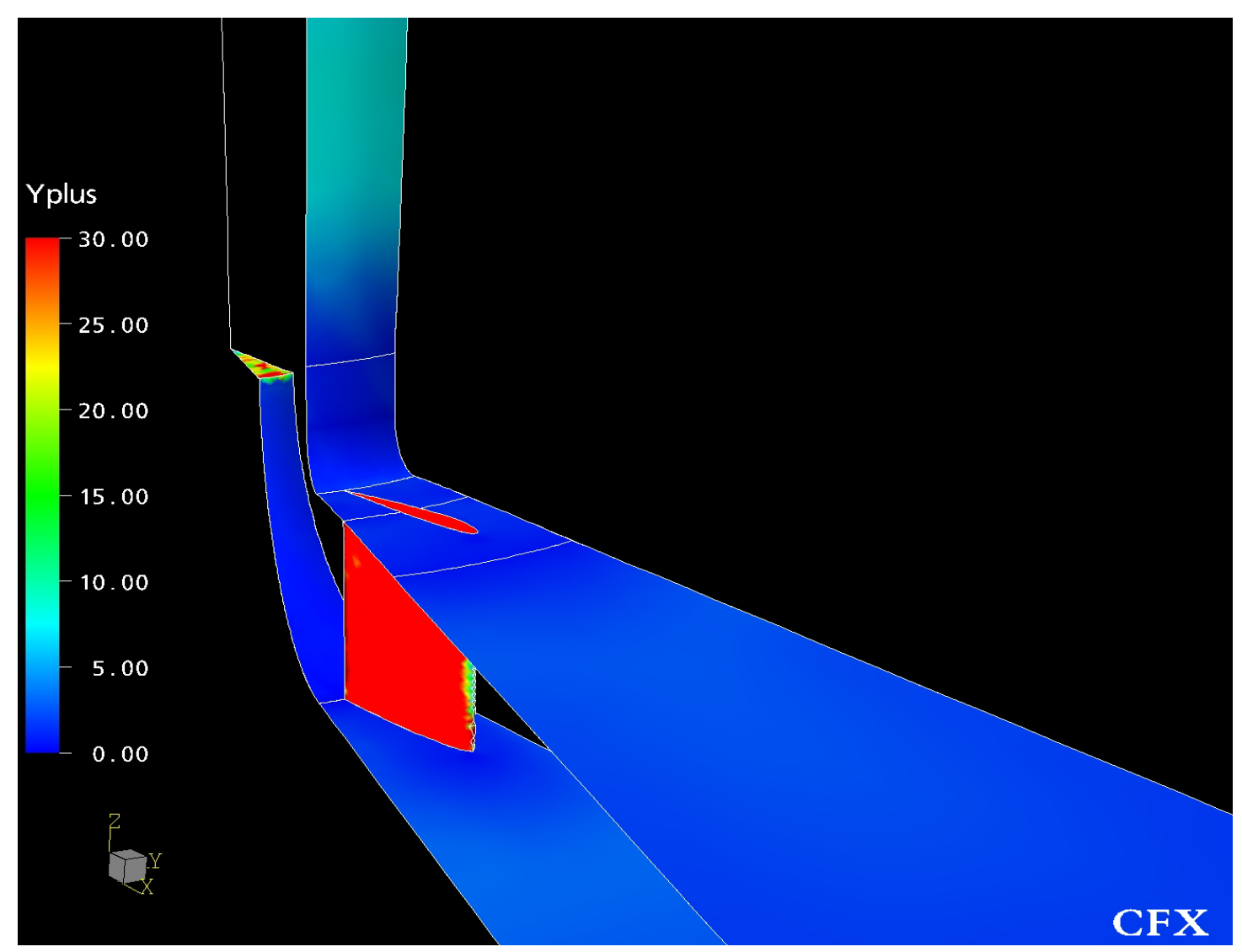

Figure 3-6 $-y^{+}$values on the CFD model 


\subsubsection{BOUNDARY- AND STARTING CONDITIONS}

\section{Turbulence model}

Feasibility studies were done from Lacovides et al. (1991); Chuang et al. (1994); Menter (1994); Rumsey et al. (1995); Rao et al. (1998); Suga et al. (2001) and the CFX Help file to determine which application would be best suited for this project. The Reynolds Stress Models (RSM) were considered briefly, but due to the unstable nature of these models as well as the longer computational times required, the focus was to rather find an adequate two-equation turbulence model.

While standard two-equation models provide good predictions for many flows of engineering interest, there are applications for which these models fail. The $k-\varepsilon$ models for instance are good in determining free stream conditions, but are inaccurate in simulating flow separation from smooth surfaces. The $k$ - $\varepsilon$ model usually gives an optimistic result, by under predicting flow separation and predicting its onset too far downstream. The $k$ - $\omega$ model specialises in determining the separation points and is advantageous in near-wall treatment for low Reynolds numbers. The model is however very sensitive to the freestream conditions which are affected by the choice of inlet conditions.

Generally problems occur with the two-equation models with:

- Flows with sudden changes in the mean strain rate.

- Flows over curved surfaces.

- Flows in rotating fluids.

- Flows with boundary layer separation.

This project has all four problem areas mentioned above. The weaknesses of standard two-equation models are well known and have resulted in a number of modifications and model enhancements (Chuang et al., 1994; Menter, 1994; Rao et al., 1998 and Suga et al., 2001). The problem is however that most of these modifications are not found in the CFX package and most of these modifications are application specific. However, a major improvement in terms of flow separation predictions has been achieved by the $k$ - $\omega$ based SST model. It accounts for the transport of the turbulent shear stress and gives highly accurate predictions of the onset and the amount of flow 
separation under adverse pressure gradients. The SST turbulence model makes use of blending functions between the $k$ - $\varepsilon$ and the $k$ - $\omega$ models based on the distance to the nearest surface and on the flow variables.

The SST turbulence model was finally chosen as the model to be used for all simulations. Comparisons with the answers of the RSM and other turbulence models (not shown in this document for brevity) indicated that the answers were consistent between the SST and the RSM, but that the SST required less time to solve. The other models also compared well in the test cases, although they were not significantly faster to solve. However, since the wake prediction from the IGVs could not be verified at that stage the SST model remained as the best option.

\section{Inlet}

The inlet of the SCPP is on the outer perimeter of the deck. Here the velocity of the flow is so low that any disturbances could affect the turbulence at the inlet. The inlet is also so large that the disturbance could be very local. Any such disturbance would fortunately be smeared out due to the nature of the model. But it does mean that it is very difficult to predict the turbulence intensity and length scale at the inlet. Therefore the default intensity was used and the length scale calculated automatically. When this option is enabled the intensity is set to $\mathrm{I}=\mathrm{u} / \mathrm{U}=0.037$, which is an approximate value for internal pipe flow. Then the $k$ and $\varepsilon$ values are calculated with the following equations

$$
\begin{aligned}
& k_{\text {inlet }}=\frac{3}{2} I^{2} U^{2} \\
& \varepsilon_{\text {inlet }}=\rho C_{\mu} \frac{k^{2}}{\mu_{t}}
\end{aligned}
$$

where

$$
\mu_{t}=1000 I \mu
$$

The mass and momentum are calculated by specifying the static pressure at the inlet. The reason is that it gives the most accurate answers, by assuming that the static pressure is uniform over the entire inlet (-1.08 $\mathrm{Pa}$ for the higher deck and $-1.64 \mathrm{~Pa}$ for the lower deck). If mass flow was used, it would have been more accurate, but 
defining mass flow at the inlet and outlet causes instability in the solver. The reasons for having to use mass flow at the exit are explained in the outlet section.

The static pressure values were determined by the average velocity through the inlet, but the exact answers were not crucial since the results obtained were very insensitive to the choice of static pressure at the inlet. However, to calculate the static pressures, the assumption is made that the total pressure at the inlet is zero, this gives the equation

$$
P_{\text {stat }}=-\frac{1}{2} \rho V_{a v e}^{2}
$$

where

$$
V_{\text {ave }}=\frac{Q}{A_{\text {perimeter }}}=\frac{Q}{2 \pi \cdot r_{\text {perimeter }} \cdot h}
$$

CFX defines inlets and outlets very strictly, in that they only allow mass transfer across the boundary in one direction. It was found that defining the inlet of the SCPP as an inlet to CFX, caused convergence problems in the solver for some of the cases. Since only the static pressure is defined at the inlet, the inlet could be defined as an opening. This made the solution system the most stable.

\section{Outlet}

At the outlet, defined as an outlet, the mass flow rate was specified. The reason for using the mass flow rate is primarily that the flow is swirling and has a variable total velocity over the section. This makes it a bad assumption by specifying an exit static pressure or normal velocity.

Since the air was specified to be at the standard air and pressure, CFX calculates the density to be $1.284\left[\mathrm{~kg} \cdot \mathrm{m}^{-3}\right]$. Since the volume flow rate is known from the experiments, the mass flow rate is easily determined by multiplying the density with an $18^{\text {th }}$ of the total volume flow rate. This gives a result of $0.356667\left[\mathrm{~kg} \cdot \mathrm{s}^{-1}\right]$ for the experimental rig and $13889\left[\mathrm{~kg} \cdot \mathrm{s}^{-1}\right]$ for the full scale SCPP (Von Backström, Bernhardt and Gannon, 2003). 


\section{Wall}

All the faces, with the exception of the periodic pair, are simulated as no-slip (stationary) walls. This means that the wall is stationary and that $\mathrm{V}_{\mathrm{w}}=0$.

\section{Periodic Pair}

The two sides of the $18^{\text {th }}$ section are simulated as a periodic pair. A periodic pair means that the conditions on the one side correspond exactly to the conditions of the other side. Thus the values on one side are mapped onto the other side.

\section{Time step}

A good estimation of the time step is to use the time that it would take a particle from when it enters the flow domain until it leaves the flow domain. This may be easy enough to calculate for problems where the through-flow velocity is constant, but in this project the calculation of this duration is far more time consuming and unreliable than letting CFX compute the time step automatically.

The length scales in CFX that are used to compute the time step are:

$$
L_{v o l}=\sqrt[3]{V} \quad L_{e x t}=\min \left\{L_{x}, L_{y}, L_{z}\right\}
$$

where $V$ is the domain volume and $\mathrm{L}_{\mathrm{x}, \mathrm{y}, \mathrm{z}}$ the extent of the domain. The velocity scales that are used are:

$$
U_{b c}=\max \left|\overline{\mathbf{U}}_{b c}\right| \quad U_{\text {node }}=\left|\overline{\mathbf{U}}_{\text {node }}\right| \quad U_{\Delta p}=\sqrt{\frac{p_{b c, \text { max }}-p_{b c, \text { min }}}{\bar{\rho}_{\text {node }}}}
$$

where $\overline{\mathbf{U}}_{b c}$ is the arithmetic average velocity on a boundary, $\overline{\mathbf{U}}_{\text {node }}$ is the arithmetic average of the nodal velocities, $p_{b c, \max }$ and $p_{b c, \min }$ are the maximum and minimum pressure values on an open boundary and $\bar{\rho}_{\text {node }}$ is the arithmetic average nodal density. Then the timescale is calculated by:

$$
\Delta t=\min \left\{t_{U} ; t_{\Delta p}\right\}
$$

where

$$
t_{U}=0.3 \frac{\min \left\{L_{v o l} ; L_{e x t}\right\}}{\max \left\{U_{b c} ; U_{n o d e}\right\}} \quad t_{\Delta p}=0.3 \frac{\min \left\{L_{v o l} ; L_{e x t}\right\}}{U_{\Delta p}}
$$


The automatic timescale of CFX is generally conservative, but it ensured that no numerical instabilities were caused due to the timescale.

\subsubsection{ERRORS DUE TO ADVECTION SCHEMES}

CFX makes use of a multigrid accelerated incomplete lower upper (ILU) factorisation technique for solving discrete systems of linearised solutions (CFX Help and Demmel, 1997). The solutions of the simulations are prone to a degree of error due to the numerical approximation schemes that are used. Fortunately these errors can be identified and reduced by understanding the nature of the problem.

\section{Numerical diffusion}

Numerical diffusion normally occurs with an odd-number order scheme. Since the highest order CFX can simulate is 2 , the diffusion would occur with the $1^{\text {st }}$ order analyses. For structured grids, the amount of diffusion is dependent on the orientation of the flow relative to the grid. This makes the unstructured grid more advantageous, since the amount of diffusion is not dependent on the flow direction.

The effect of numerical diffusion is that the flow appears to be smeared out. For instance a step function would end up looking like a sigmoid curve (illustrated in Figure $3-7)$. In this project the $1^{\text {st }}$ order advection scheme was not used, because CFX in the $1^{\text {st }}$ order did not capture the wake formation or the boundary layers adequately. The result of this smearing is not shown here for brevity, but the results were not satisfactory.

\section{Numerical dispersion}

To eliminate numerical diffusion, higher order advection schemes are used. The higher order advection scheme in CFX can be anything between 1 and up to 2 . The $2^{\text {nd }}$ order advection scheme is however not always entirely accurate, since it can lead in some cases to numerical dispersion. Numerical dispersion is when the results overshoot; this is also illustrated in Figure 3-7. 


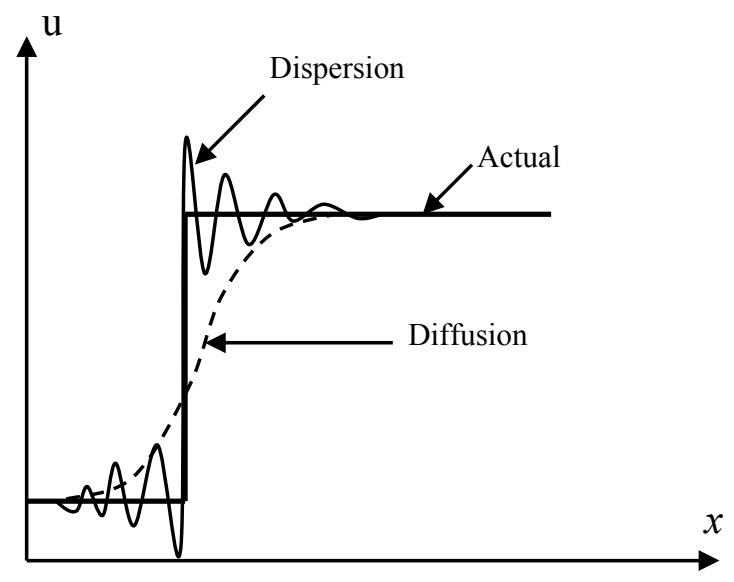

Figure 3-7 - Numerical errors

The best results were obtained by using the $2^{\text {nd }}$ order scheme, instead of $1^{\text {st }}$ order or a blend of the two schemes. The results from the $2^{\text {nd }}$ order were satisfactory even though a small quantity of dispersion was prevalent (indicated in Figure 4-15). 


\section{CHAPTER 4:RESULTS}

\subsection{INTRODUCTION TO THE SYNTHESIS OF THE RESULTS}

The two previous chapters explained what was done in the project to obtain the results. This chapter mainly explains how the CFD and EFD results were interpreted and compared with each other, but give error analyses where possible.

The sections in this chapter pertaining to the experimental work are the basis for validating the numerical software. This project, however, would not be useful if only the experimental results were compared with simulated results and it could not be used for the full scale geometry plant. Therefore the final section of this chapter extends the numerical simulation to a proposed full scale geometry and makes predictions on the loss coefficient and performance of the transition section.

The final section of this chapter contains the correlations that predict the loss and turning angle through the transition section into the turbine. The correlations are defined in terms of two independent variables, namely the IGV stagger angle and the collector roof height. These results complete the primary objective of this project. 


\subsection{Five-Hole Probe Calibration}

\subsubsection{RESULTS}

The procedure to calibrate the five-hole probe experimentally was described in $\S 2.3$. The results of the calibration and the mathematics behind it are explained in this chapter. The pressure readings are defined by four pressure coefficients. The pressure coefficients for the five-hole probe are defined as follows (refer to Figure 2-4):

$$
\begin{aligned}
& \bar{P}=\frac{P_{2}+P_{3}+P_{4}+P_{5}}{4} \\
& C p_{\text {centre }}=\frac{P_{1}-P_{s}}{P_{\text {tot }}-P_{s}} \\
& C p_{\text {average }}=\frac{\bar{P}-P_{s}}{P_{\text {tot }}-P_{s}} \\
& C p_{\text {YAW }}=\frac{P_{2}-P_{3}}{P_{1}-\bar{P}} \\
& C p_{\text {PITCH }}=\frac{P_{4}-P_{5}}{P_{1}-\bar{P}}
\end{aligned}
$$

The figures below illustrate the calibration maps of the centre and average pressure coefficients. These figures show that the probe is highly symmetrical on the yaw- and pitch axes. As seen below the calibration values are slightly offset from the pitch axis, this is due to the stem the probe is attached to.
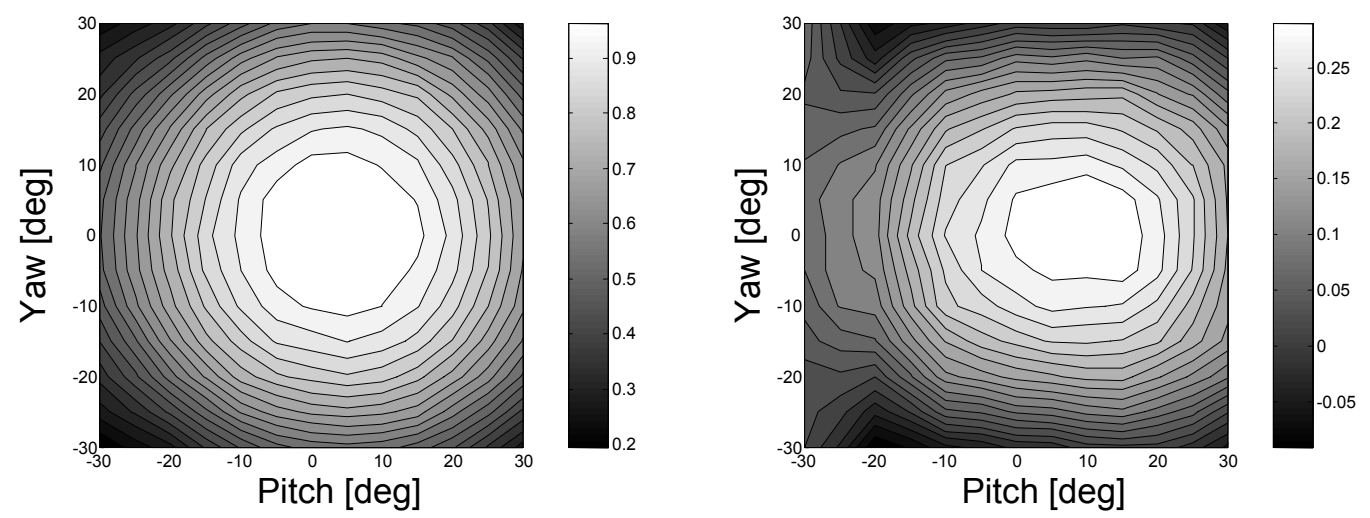

Figure 4-1 - $C p_{\text {centre }}$ (left) and $C p_{\text {average }}$ (right) Calibration Maps

The yaw- and pitch coefficients have sloping graphs as shown in the figures below 

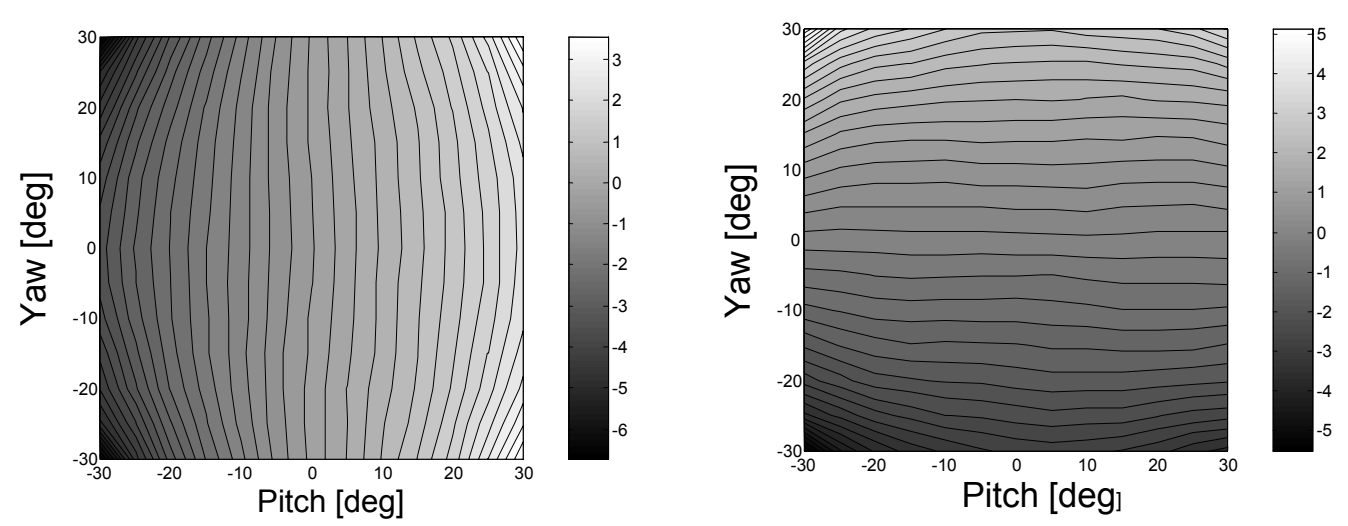

Figure 4-2 - $C \mathrm{p}_{\text {pitch }}$ (left) and $\mathrm{C} \mathrm{p}_{\text {yaw }}$ (right) Calibration Maps

From the pitch- and yaw calibration maps together, the pitch- and yaw angles can be determined. This is achieved by using the pitch- and yaw coefficients, and then superimposing their intersections with their corresponding maps. To illustrate, consider that for a single $\mathrm{Cp}_{\text {pitch }}$ or $\mathrm{Cp}_{\text {yaw }}$ value, a curve is defined on its corresponding map. These curves will run almost perpendicularly with each other and will intersect at the pitch- and yaw angle of the probe if the flow angle is within range. The figure below demonstrates such an example

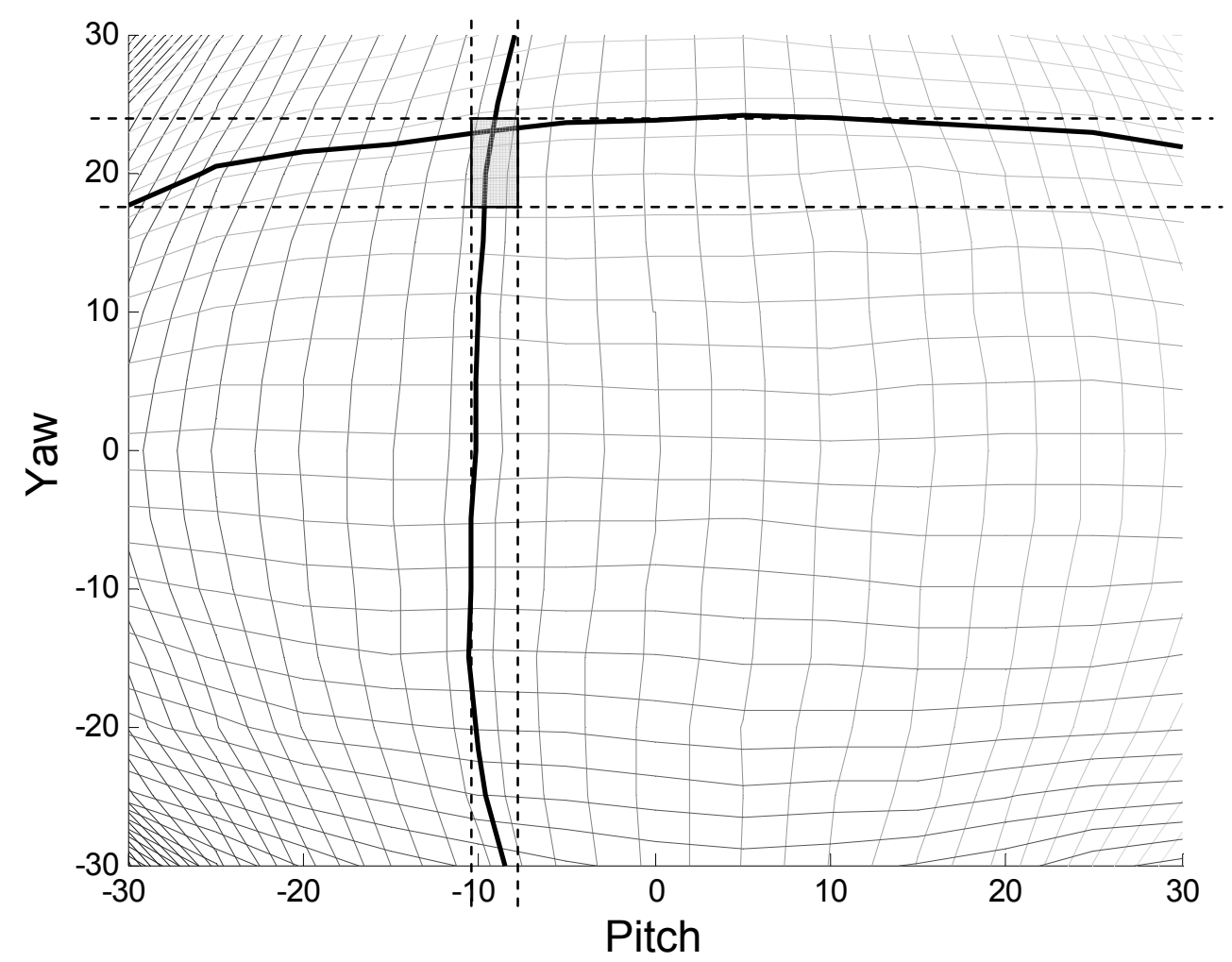

Figure 4-3 - Angle optimisation setup 
On the figure above the result of the $\mathrm{Cp}_{\text {pitch }}$ and $C \mathrm{p}_{\text {yaw }}$ intersections are shown in the thick black lines, the other lines are the contours of the two maps superimposed. The result desired is at the intersection of these two thick lines. To determine the intersection point, an optimization procedure is required.

The most effective way to determine the intersection point is by defining the maxima and minima of the boundaries. i.e. the intersection will lie between the global maxima and minima of the curves, thus by determining the minimum and maximum yaw angle of the horizontal line and the minimum and maximum pitch angle of the vertical line, the first set of boundaries are found. The window is indicated on the figure above and shown in the figure below.

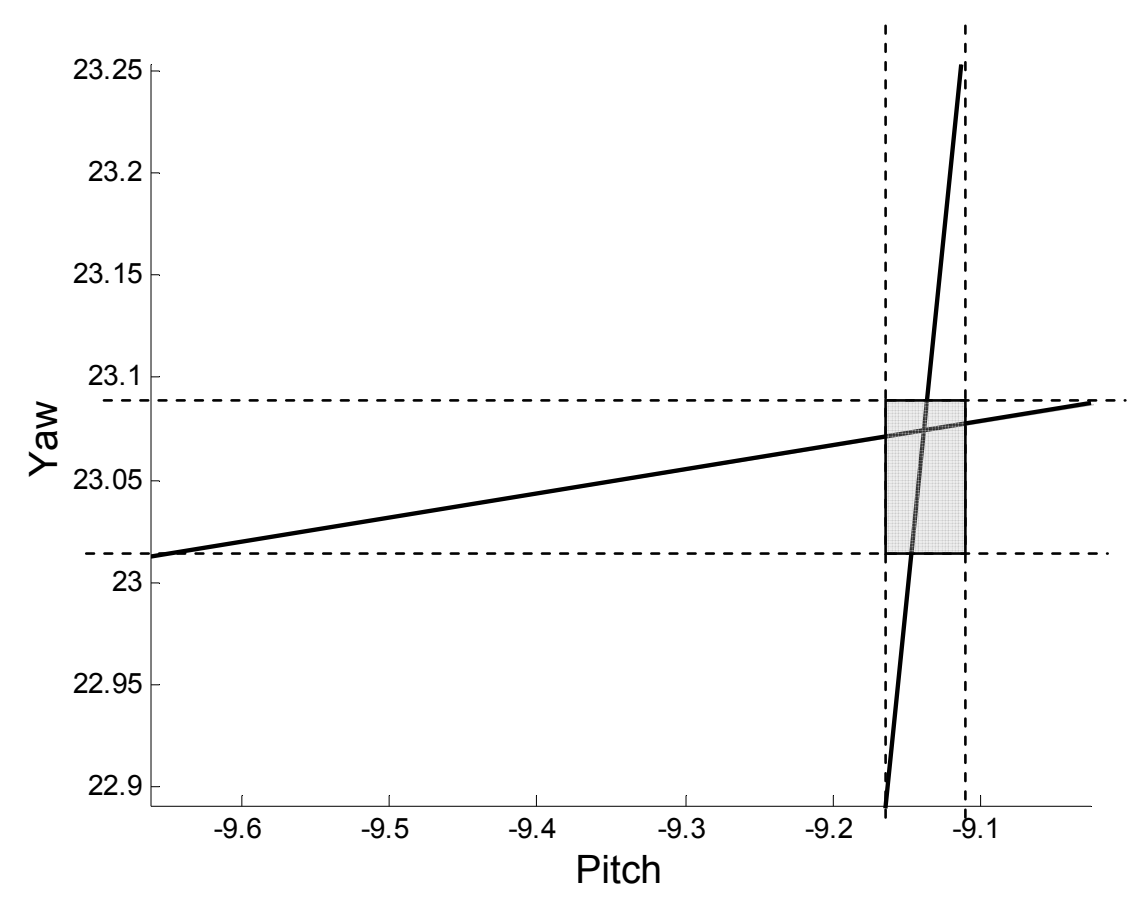

Figure 4-4 - Angle optimisation $1^{\text {st }}$ step

Already the angle is accurate to within $0.1^{\circ}$ for the yaw and about $0.05^{\circ}$ for the pitch angle. Very good for a first iteration, but more extreme gradients may be found, thus the process is repeated, by calculating the maxima and minima on the new boundaries, so that new window can be found as shown in the figure above and detailed in the figure below. 


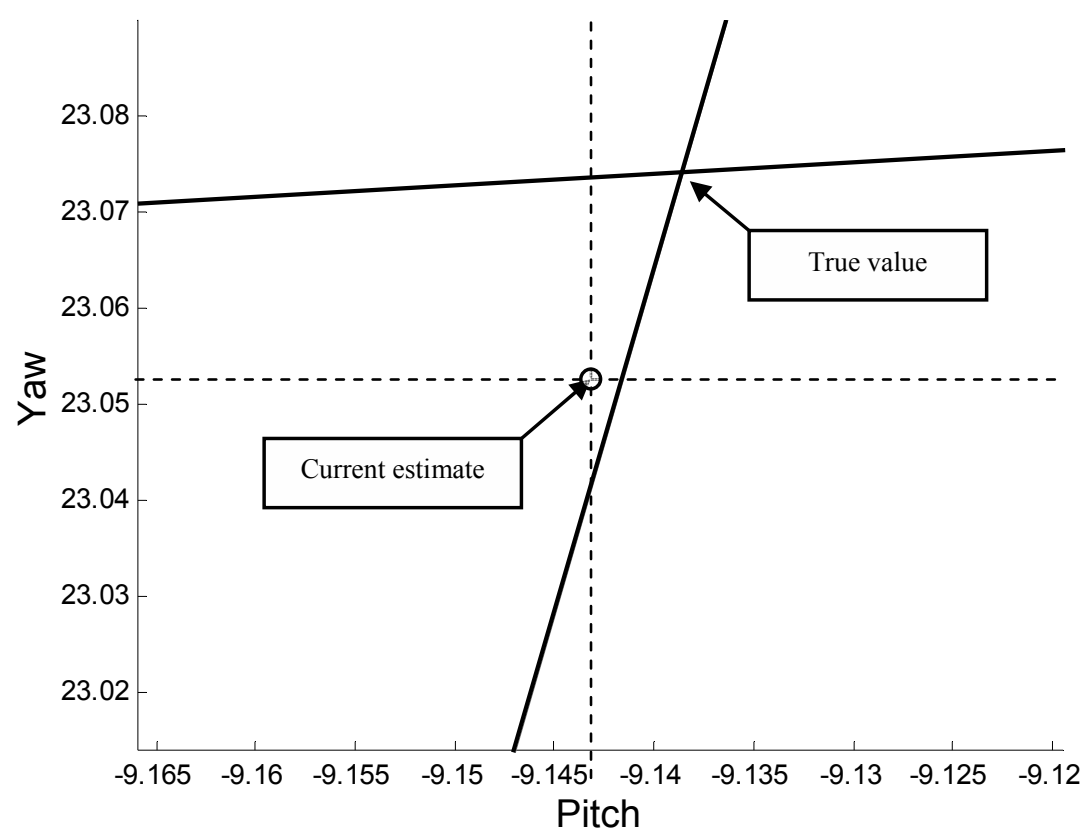

Figure 4-5 - Angle optimisation $2^{\text {nd }}$ step

This process is repeated until convergence is obtained. The result is chosen to be halfway between the boundaries; therefore the greatest possible error is half of the tolerance. It normally takes 3 iterations to determine the angle to within $0.005^{\circ}$. The case illustrated in the figures above took 4 iterations using cubic interpolation to determine that values of $\mathrm{Cp}_{\text {pitch }}=-1.1997$ and $C \mathrm{p}_{\text {yaw }}=2.0700$ give a pitch angle of $9.185^{\circ}$ and a yaw angle of $23.085^{\circ}$. The discrepancy of the values in the figure to these results is because the figure plotted the thick lines as linear sections instead of a cubic spline. It is not due to a calculation error.

Once the yaw and pitch angles are known, the $C \mathrm{p}_{\text {centre }}$ and $C \mathrm{p}_{\text {average }}$ values can be obtained and converted to total and static pressures. To convert the coefficients to static- and total pressure equation (4-2), (4-3), (4-4) and (4-5) are rearranged to get:

$$
\begin{aligned}
& P_{\text {stat }}=\frac{C p_{\text {centre }} \cdot \bar{P}-C p_{\text {average }} \cdot P_{1}}{C p_{\text {centre }}-C p_{\text {average }}} \\
& P_{\text {tot }}=P_{\text {stat }}+\frac{P_{1}-P_{\text {stat }}}{C p_{\text {centre }}}
\end{aligned}
$$


Once the angles and dynamic pressure are found, the velocities are calculated using Bernoulli's principle. These calibration tables and methods were implemented into the sampling program for the five-hole probe.

The averages and variances of the coefficients during calibration were determined by adding the new weighted measured value to the current average value. The sampling function recalls how many samples have been taken at any specific co-ordinate and then adjusts the values according to the following derived equations

$$
\begin{aligned}
& \bar{X}^{\text {new }}=\frac{\bar{X}^{\text {old }} \cdot n+X}{n+1} \\
& S^{\text {new }}=\sqrt{\left(S^{\text {old }}\right)^{2} \frac{n-1}{n+1}+\frac{\left(\bar{X}^{\text {new }}-X\right)^{2}}{n(n+1)}}
\end{aligned}
$$

$\mathbf{X}$ is the variable, $\mathbf{n}$ the number of samples and $\mathbf{S}$ the variance of the variable. The derivations for these equations are shown in $\S$ A.4.3.

\subsubsection{ERROR ESTIMATION}

Closer inspection of equation (4-9) shows that the equation is not absolutely accurate for the first few samples, since the average for the variables change as their new values are determined. However this erroneous variance converges rapidly to the true variance. When more than 5 samples with these low variances are taken, the difference becomes indistinguishable.

Table 4-1 - Variance of probe coefficients with $95 \%$ confidence at $16 \mathrm{~m} / \mathrm{s}$

\begin{tabular}{|c|c|c|c|}
\cline { 2 - 4 } \multicolumn{1}{c|}{} & Range & Maximum variance & Average variance \\
\hline $\mathbf{C p}_{\text {centre }}$ & 0.8 & 0.0047 & 0.0026 \\
\hline $\mathbf{C p}_{\text {average }}$ & 0.3 & 0.0061 & 0.0025 \\
\hline $\mathbf{C p}_{\text {pitch }}$ & 8 & 0.0743 & 0.0100 \\
\hline $\mathbf{C p}_{\text {yaw }}$ & 10 & 0.0771 & 0.0010 \\
\hline
\end{tabular}

The pressures were calibrated relative to a $5 \mathrm{~mm}$ pitot tube. Therefore the five-hole probe gives the same values as the pitot tube for a large range of velocities, but it 
should be treated sceptically for low velocities. The measured values were verified to be amply accurate for velocities as low as $8 \mathrm{~m} / \mathrm{s}$. Below $8 \mathrm{~m} / \mathrm{s}$ the Reynolds number based on the probe diameter is below 1000, hence friction effects become substantially influential and henceforth the probe requires recalibration to measure these low velocities accurately. In this thesis there are a few cases where velocities below $8 \mathrm{~m} / \mathrm{s}$ were measured, and then large variations were found in the measurements. These large variations were averaged from a few samples and gave reasonably accurate results. It was not worthwhile to recalibrate the probe for low velocities or to take many samples in these regions in the hope of obtaining more accurate results.

The variations were initially obtained for $16 \mathrm{~m} / \mathrm{s}$. Experience of the probe behaviour recalls that the higher the velocity, the lower the variance, and the lower the velocity the higher the variance. The only discernable cause of the variance is due to the variance of the pressure transducers. The investigation of the transducer variance can be found in $\$ 2.2 .5$.

To analyse the effect of this variance on the five-hole probe results, the variances of the pressure transducers are converted into variance for the $\mathrm{Cp}$-values. To determine the variance the error estimation technique described in Granger (1988) is used.

If $F=f\left(X_{1}, X_{2}, \ldots, X_{n}\right)$ and the error of $\mathbf{F}$ is $\mathbf{B}_{\mathbf{F}}$, then the error for $\mathbf{F}$ can be written as:

$$
B_{F}^{2}=\sum_{i=1}^{n}\left(\frac{\partial F}{\partial X_{i}} B_{X_{i}}\right)^{2}
$$

Where $\mathbf{B}_{\mathbf{X} \mathbf{i}}$ is the error of any variable, $\mathbf{X}_{\mathbf{i}}$, in $\mathbf{F}$. Now equation ( 4-4) for $C \mathrm{p}_{\text {yaw }}$ is used and the error, or variance, called $\mathbf{S}$. The pressure transducers and the average pressure have the same variance, henceforth the variance of $\mathrm{Cp}_{\text {yaw }}$ can be written as:

$$
S_{C p_{Y A W}}=S_{P} \sqrt{\frac{2}{\left(P_{1}-\bar{P}\right)^{2}}+2 \cdot \frac{\left(P_{2}-P_{3}\right)^{2}}{\left(P_{1}-\bar{P}\right)^{4}}}
$$


where $\mathrm{S}_{\mathrm{P}}$ is the variance of a pressure transducer (the derivations are shown in $\S$ A.4.3). Now similarly for $\mathrm{Cp}_{\text {pitch: }}$ :

$$
S_{C p_{P I C H}}=S_{P} \sqrt{\frac{2}{\left(P_{1}-\bar{P}\right)^{2}}+2 \cdot \frac{\left(P_{4}-P_{5}\right)^{2}}{\left(P_{1}-\bar{P}\right)^{4}}}
$$

It can be seen that the lower pressures resulting from the lower velocities, give larger variances for the yaw and pitch angles, since the denominators decrease. It can also be seen that when the probe is facing into the flow, the magnitude of right-hand term decreases as the difference between $P_{1}$ and the average pressure increases. This begs the question of what the magnitudes of these changes between the left-hand term and right-hand term relative to each other are.

When the pressures become low and the probe is at an angle, the magnitude of the difference below the equation decreases thus amplifying the variance. Using known pressure values, it becomes evident that the amplification of the right-hand term does not become notable within the used range of angles. The denominator is usually in the order of $10^{2}$. Therefore the $4^{\text {th }}$ power of the denominator in the right-hand term reduces the term to an insignificant magnitude in comparison to the left-hand term. Therefore the right-hand term can be ignored for this application, and the conclusion made that the angular variance is not affected by the probe-head orientation.

The measurement errors are difficult to evaluate since the calibration maps are not defined by equations, but by experimental maps. Hence known experimental pressure readings for various angles with its variations were used. The result was surprisingly consistent with the assumption that the flow angles can be ignored. This conservative result is shown in Figure 4-6. 


\section{Variance of Measurement Results}

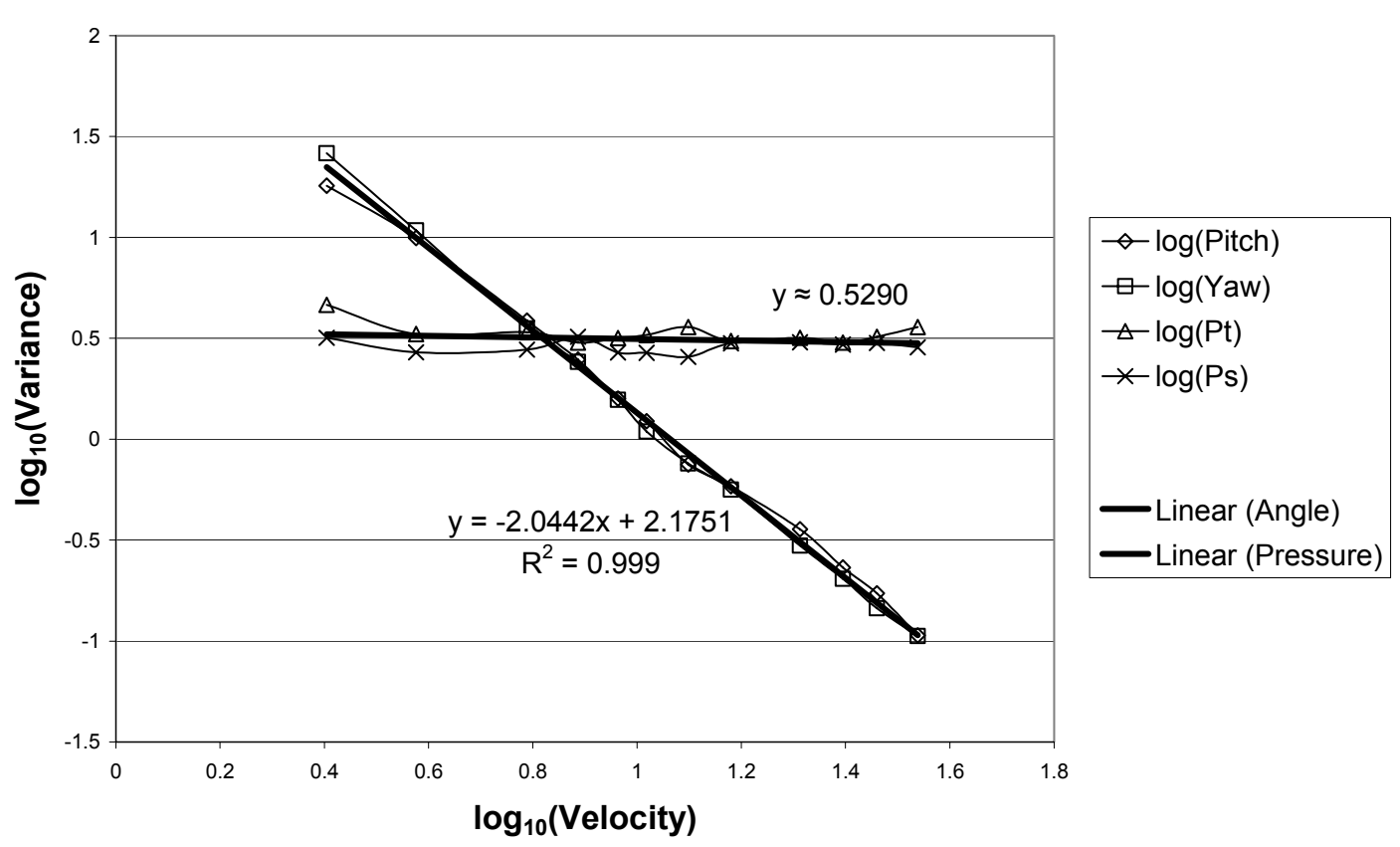

Figure 4-6 - Variance of measurements in the five-hole probe

The variances of the static- and total pressures are insensitive to both probe orientation and flow velocity. The pressures have approximately 1.96 times the variance of one of the pressure transducers, namely 3.381 Pa. Although this variance was calculated conservatively, it is too large for determining the total pressure. In this thesis a correlation between the loss coefficient predicted by the experiments and CFD is required, and since the total pressure is near zero, such a variance could change the result by as much as $100 \%$. To reduce the variance it is therefore necessary that more samples are taken at the same point. The variance could then be divided by the square root of the number of samples, or written out.

$$
S_{\text {pressure }}=\frac{3.381}{\sqrt{n}}
$$

where $\mathbf{n}$ is the number of samples at that point.

To reduce the variance to less than $1 \mathrm{~Pa}, 12$ samples have to be taken at each point. This was too expensive in terms of time, and hence the average number of samples taken at each point was 6 instead, thus reducing the total experimentation time by 
about $40 \%$ and the increasing variance to $1.38 \mathrm{~Pa}$. However, more than 6 samples were taken at the areas that were seen to have a larger variance.

The yaw and pitch angles are insensitive to probe orientation as predicted, but vary with the flow velocity. The angles have the same variance and therefore both can be estimated using the equation obtained from Figure 4-6, namely:

$$
S_{\text {angle }} \approx \frac{150}{V_{\text {tot }}^{2}}
$$

Hence the error for the angle can be estimated by knowing the total velocity. If more than one sample is taken, as is required for the total pressure, the equation can be changed to:

$$
S_{\text {angle }} \approx \frac{150}{\sqrt{n} \cdot V_{\text {tot }}^{2}}
$$

Care was taken to set the yaw offset angle as accurately as possible, but even then the offset cannot be set with confidence to within $0.5^{\circ}$ of the desired offset yaw angle. Furthermore the probe sleeve is supported at the one end only, and could be deflected at the tip by the flow, hole or gravity causing pitch angle deflections as great as $2^{\circ}$.

The accuracy of the five-hole probe is adequately consistent, but with the current probe-bracket the flow angles cannot be measured as accurately as the probe is capable of. Therefore when experimenting, a margin of $1^{\circ}$ was allowed for the yaw angles and $2^{\circ}$ for the pitch angles. If any of the numerically simulated results are within this tolerance, then it can be safely assumed to have the same value as the experimental value. Hence the bracket error defines the lower limit of the angular tolerance for the five-hole probe as it is set up currently, and not equation (4-15), although it is useful to know how accurately you should be able to measure if better brackets are used.

The majority of velocities in this thesis averaged at $15 \mathrm{~m} \cdot \mathrm{s}^{-1}$ and an average of 6 samples per co-ordinate; therefore using equation (4-15) and (4-13), the angular 
variance is determined to be $0.24^{\circ}$, or $1^{\circ}$ for yaw and $2^{\circ}$ for pitch. The pressures are expected to have a variance of $1.38 \mathrm{~Pa}$ and due to the calibration a maximum offset error of $2.2 \mathrm{~Pa}$ is also allowed. This gives a probable error for the pressures of about $3.58 \mathrm{~Pa}$.

Unfortunately this leaves large uncertainties relative to the typical magnitude of the total pressure being measured. The average total pressure at the top row is approximately $-4.5 \mathrm{~Pa}$, hence the error could be as great as $80 \%$. However this is statistically unlikely due to the amount of samples taken, therefore errors of up to $30 \%$ are expected instead. This makes it somewhat precarious to directly compare the experimental results with the CFD results for the total pressure; however at least the order of magnitude of the loss could be compared. The results are shown in Table 4-3. 


\subsection{Outer Wall Pressure}

This experimental procedure was time consuming, because the sampling program had to pause after changing a channel on the switch box, to allow the new pressure in the output tube to stabilize. This meant that to keep the experimenting time reasonable, the average of only 3 readings per hole were used. The variances on the holes are less than $1 \mathrm{~Pa}$, which gives a maximum error of $2 \%$ for the sampled pressures. All of these results are found in Appendix B.5 to B.8, where the EFD results are compared with the CFD results.

In all four test rig configurations the first row has a discrepancy that does not follow the trend simulated or indicated by the other rows. Row 5 is supposed to have the same results as row 1, since they are at the same azimuthal co-ordinate relative to the IGVs. Row 5 does not predict this discrepancy, and hence it can be concluded that it is a local geometric error in the experimental rig. The discrepancy is shown by the circle in the figure below.

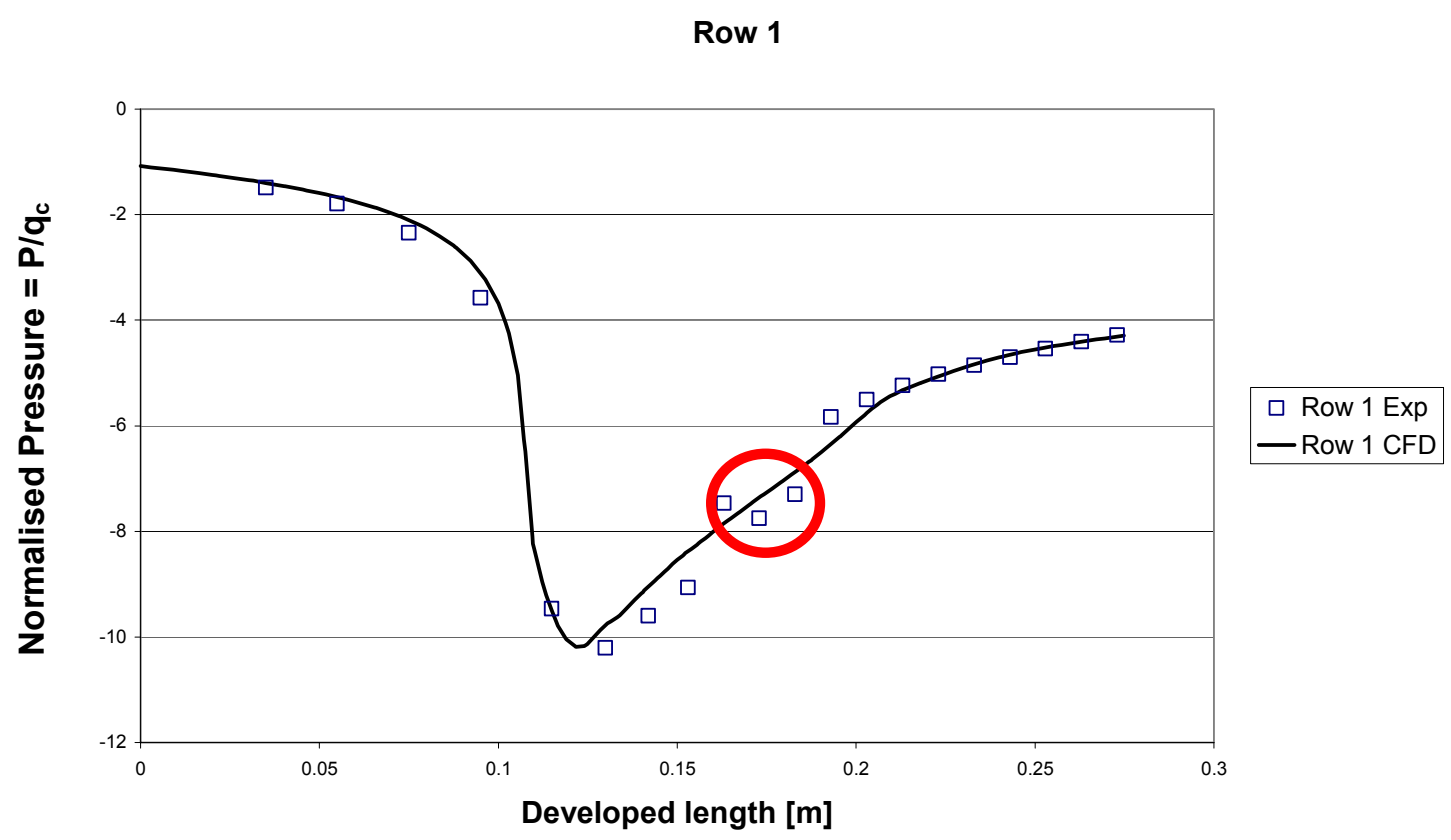

Figure 4-7 - Outer wall pressure; IGV angle $22.5^{\circ}$ and deck height $260 \mathrm{~mm}$

The CFX results have a tendency to slightly under-predict the static wall pressure at the curved section of the outer wall. Beyond the curved surface, where the wall is 
straight again, CFX slightly over-predicts the wall static pressure. These discrepancies may be due to geometric inconsistencies of the experimental rig.

Generally the first four experimental points are under-predicted. However, it was shown that the flow is still partly laminar in this region as discussed in §A.1. Therefore the SST turbulence model of CFX is predicting the pressure consistently accurate in spite of simulating the wrong flow regime. To simulate a combination of laminar and turbulent flow to get these values, however, is beyond the scope of this project. 


\subsection{Transition Section Traverse}

The results of this experimentation are shown by 264 figures in Appendix B: Figures of Results, which are too many to discuss. Therefore a sample of 9 figures that are representative of all the results are shown in this section and discussed in detail. Where there are deviations to the majority of other results, mention of it is made.

Each meridional plane is called a row in the figures. For instance in the figures below contain the results for the $7^{\text {th }}$ row (the row on the far right of the experimental rig). The results are plotted using a normalised distance on the x-axis, from the hub (inner wall) to the outer wall, so that each traverse only represents the flow passage. The yaxes are also normalised using the dynamic pressure or velocity in a $900 \mathrm{~mm}$ section of the model chimney. The angles are not normalised and their units are degrees.

The azimuthal planes are named top, middle, bottom or below bottom as indicated in Figure 2-22.

\subsubsection{VELOCITY DisTRIBUTIONS AND CONTINUITY}

The following three figures show the velocity distribution in the transition section.

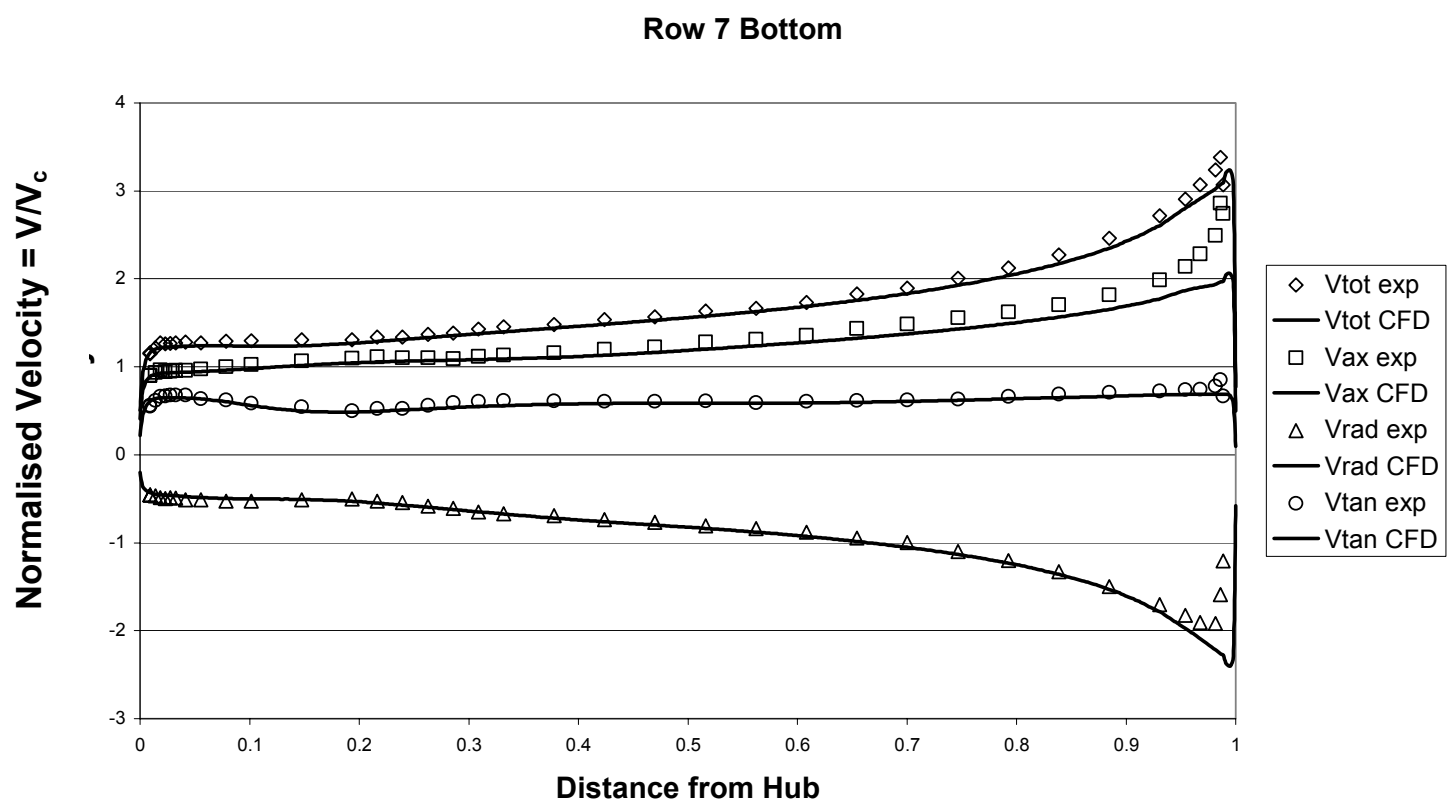

Figure 4-8 - Row 7 bottom velocity; $22.5^{\circ} 260 \mathrm{~mm}$ 
Row 7 Middle

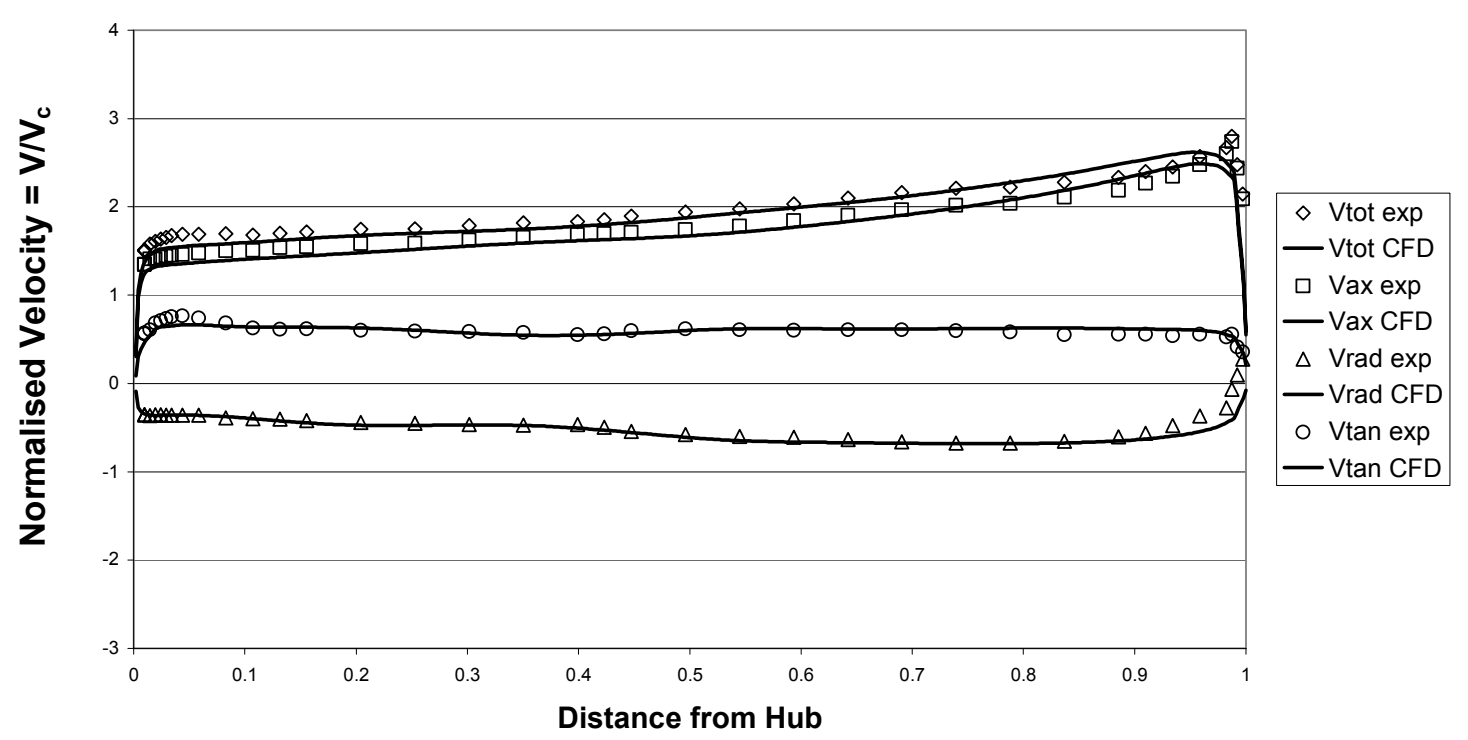

Figure 4-9 - Row 7 middle velocity; $22.5^{\circ} 260 \mathrm{~mm}$

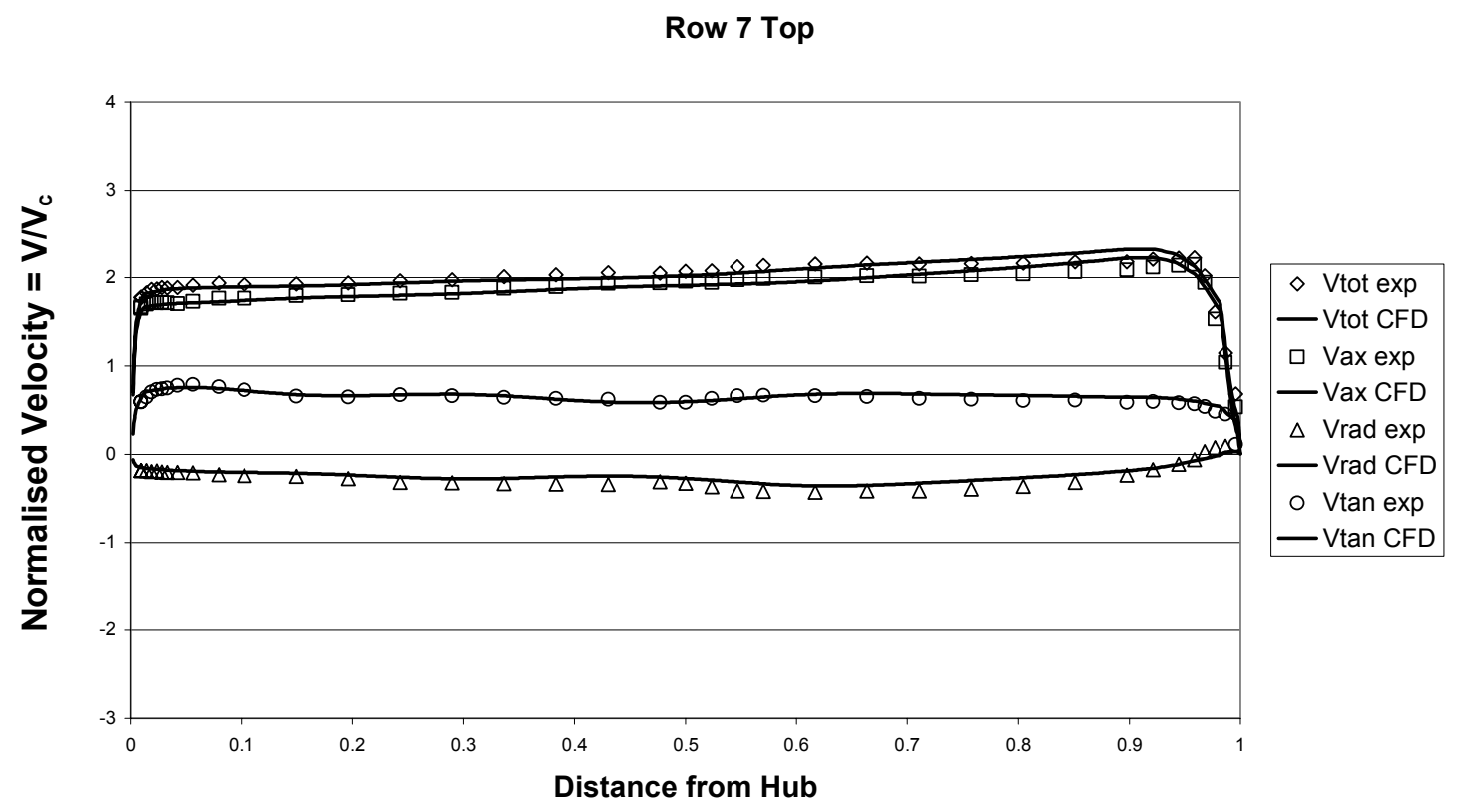

Figure 4-10 - Row 7 top velocity; $22.5^{\circ} 260 \mathrm{~mm}$

From Figure 4-8, Figure 4-9 and Figure 4-10 it can be seen that there is good agreement between the CFD and EFD results. The axial velocity towards the outer wall (distance from hub $\rightarrow 1$ ) in Figure 4-8 is under predicted by the CFD in relation to the EFD. This is due to the hole in the wall being offset by a millimetre or two. This could have been corrected by the CFD, but in order to keep the azimuthal plane 
two dimensional, this was simply accepted as an experimental error. The boundary layers seem to be predicted well enough, but the pressure readings (which are more sensitive to variations) are better for such an evaluation. Figure 4-12, Figure 4-13 and Figure 4-14 display the total- and static pressure readings, but the static pressure distribution will be discussed later. The weak wake formations are more discernible in the velocity profiles, due to fluctuating flow angles that usually accompany them.

The five-hole probe, as mentioned in $\$ 2.4 .1$, cannot get accurate readings close to the walls. The reason is because the resolution of the probe is about $2 \mathrm{~mm}$. The hole of the probe nearest to the wall, for instance, would enter the boundary layer while the rest of the probe is still in the free stream. This gives a pressure distribution that predicts the flow erroneously.

To verify the quality of the measured results, a continuity check is made. To obtain the flow rate the perpendicular velocities of each of the azimuthal planes are integrated using the trapezium law. The volume flow rate is defined as

$$
Q=\int V \cdot d A
$$

which can be written as

$$
Q=\sum V \cdot \Delta A
$$

where $\mathbf{V}$ is the velocity and $\mathbf{\Delta} \mathbf{A}$ the incremental area for integration. Each plane can be schematically defined as shown in the figure below 


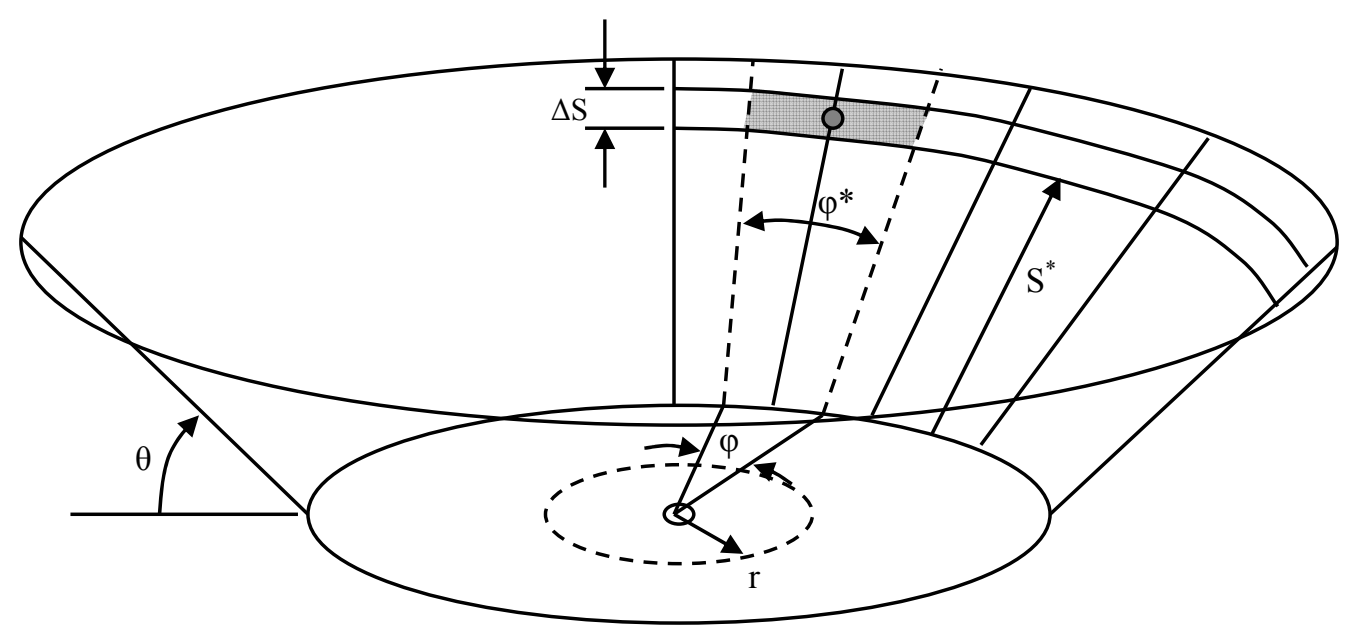

Figure 4-11 - Azimuthal plane integration schematic

From Figure 4-11 it is clear that the incremental area (highlighted in the figure) can be defined as the product of the arc length and the increment width. Therefore

$$
\Delta A=\left(S^{*} \cdot \varphi \cdot \operatorname{Cos} \theta\right) \cdot \Delta S
$$

$\mathbf{S}^{*}$ is the developed distance to the centre of the area, and $\varphi^{*}=\varphi \cdot \operatorname{Cos} \theta$. Strictly speaking the centroid of the area should be determined for $\mathbf{S}^{*}$ since the segment is trapezoidal. However $\varphi$ and $\Delta \mathrm{S}$ are small enough, especially since $\varphi$ is reduced even more by the $\cos \theta$ term, therefore assuming the centroid is halfway between the limits would not change the result notably.

The velocity normal to the plane is defined as

$$
V_{\perp}=V_{a} \cdot \operatorname{Cos} \theta-V_{r} \cdot \operatorname{Sin} \theta
$$

where $V_{a}$ and $V_{r}$ are respectively the global axial- and radial velocities. Using equation (4-17) to (4-19), the volume flow rate for each segment can be written as.

$$
Q_{\text {sector }}=\frac{1}{4} \sum_{i=1}^{n-1}\left(V_{\perp, i+1}+V_{\perp, i}\right) \cdot\left(S_{i+1}^{2}-S_{i}^{2}\right) \cdot \varphi \cdot \operatorname{Cos} \theta
$$

The total volume flow rate is calculated by simply adding all the segmental flow rates calculated in equation (4-20), which gives. 


$$
Q=\sum_{i=1}^{\text {row }-1} Q_{\text {sector }, i}
$$

Row 1 and row 7 have the same azimuthal co-ordinate and therefore the summation has the form it has in equation (4-21). The results are shown in the table below along with the anemometer readings and the input flow rates used for CFX. $\mathbf{Q}$ in equation ( 4-21) is the volume flow rate for an $18^{\text {th }}$ of the entire flow area. The configuration symbols in the table below are described in the nomenclature.

Table 4-2 - Continuity verification

\begin{tabular}{|c|c|c|c|c|c|c|c|}
\hline \multirow{3}{*}{ Configuration } & \multicolumn{6}{|c|}{ EFD $\left[\mathrm{m}^{3} \cdot \mathrm{s}^{-1}\right]$} & \multirow{3}{*}{$\begin{array}{c}\text { CFD } \\
{\left[\mathrm{m}^{3} \cdot \mathrm{s}^{-1}\right]}\end{array}$} \\
\hline & \multicolumn{4}{|c|}{ Five-hole probe } & \multirow{2}{*}{ Anemometer } & \multirow{2}{*}{ Difference } & \\
\hline & Top & Middle & Bottom & Average & & & \\
\hline $\mathbf{A}$ & 5.014 & 5.047 & 4.891 & 4.984 & 4.958 & $0.52 \%$ & 5.000 \\
\hline B & 5.072 & 4.9036 & 4.842 & 4.933 & 4.902 & $0.63 \%$ & 5.000 \\
\hline $\mathbf{C}$ & 4.974 & 5.008 & 4.889 & 4.956 & n/a & n/a & 5.000 \\
\hline D & 4.791 & 4.811 & 4.807 & 4.803 & 4.814 & $0.25 \%$ & 5.000 \\
\hline
\end{tabular}

The agreement of the average five-hole probe results with the anemometer is less than a percent for the cases being compared. The agreement is however somewhat misleading. It will be worse if the anemometers results are not averaged for many samples as shown in $\$ 2.2 .7$, or if single values from the five-hole probe were compared instead of averages. The worst agreement in continuity in the table above is in configuration B for the top and bottom plane. The results differ by $4.5 \%$, but this is an acceptable error.

\subsubsection{Pressure Distributions AND Losses}

The next three figures are the total- and static pressures that have been measured and compared with the CFD results. 
Row 7 Bottom

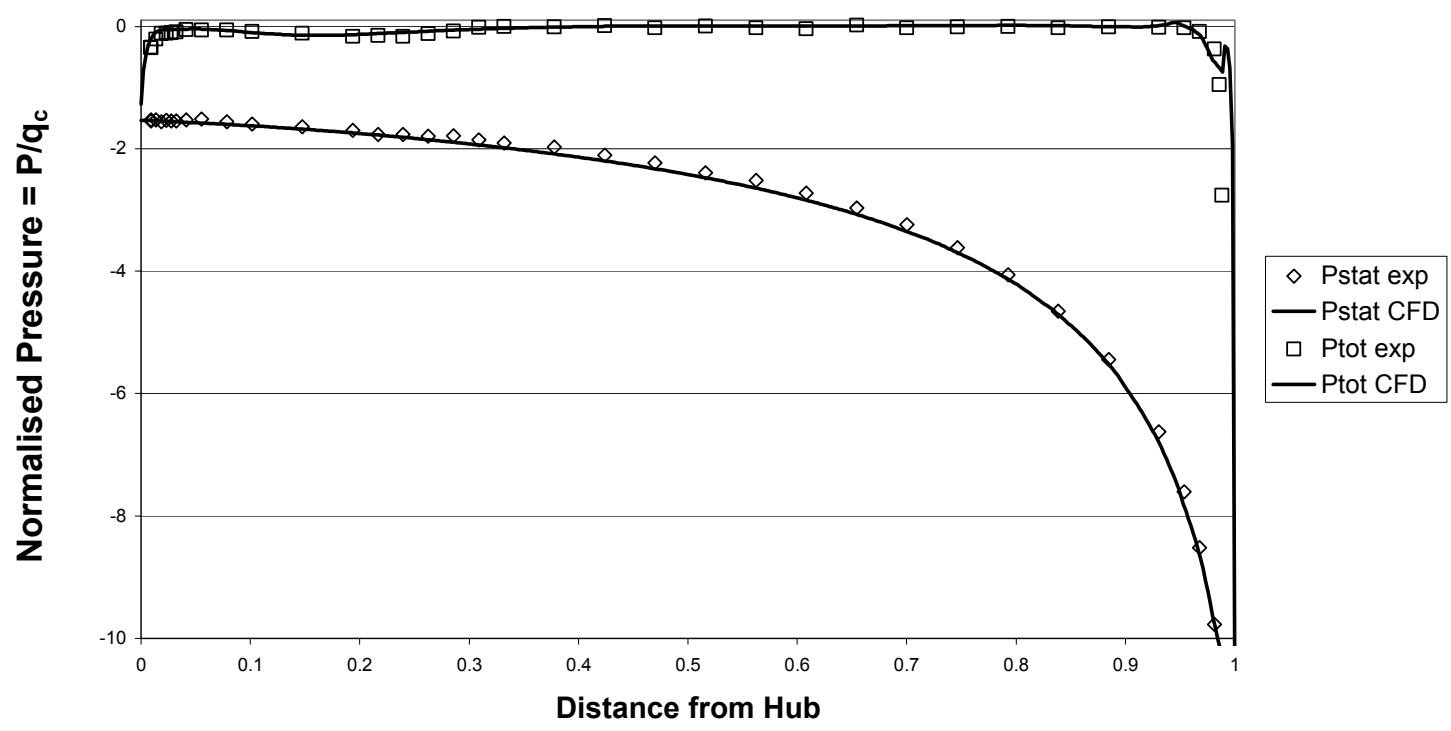

Figure 4-12 - Row 7 bottom pressure; $22.5^{\circ} 260 \mathrm{~mm}$

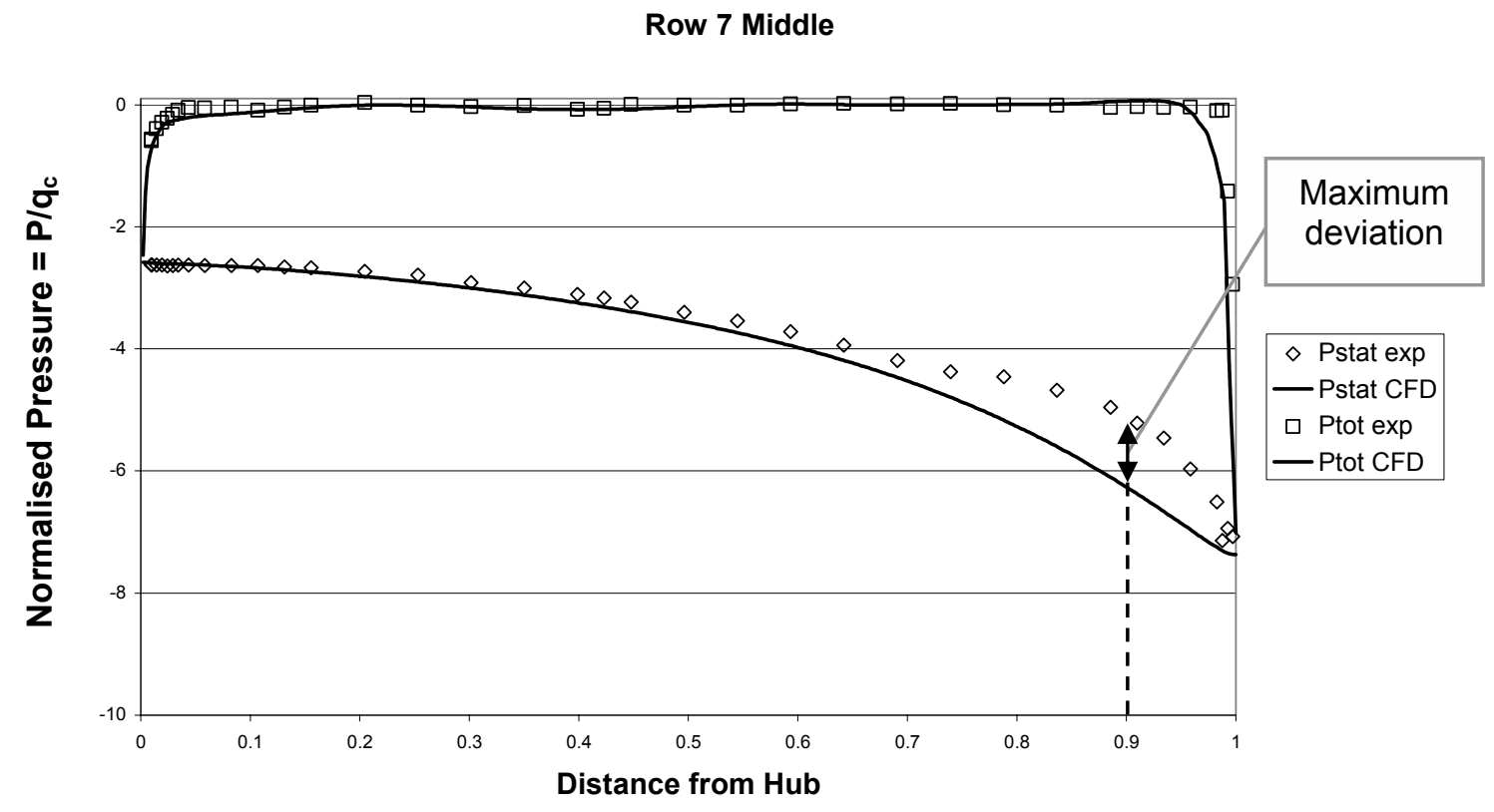

Figure 4-13 - Row 7 middle pressure; $22.5^{\circ} 260 \mathrm{~mm}$ 
Row 7 Top

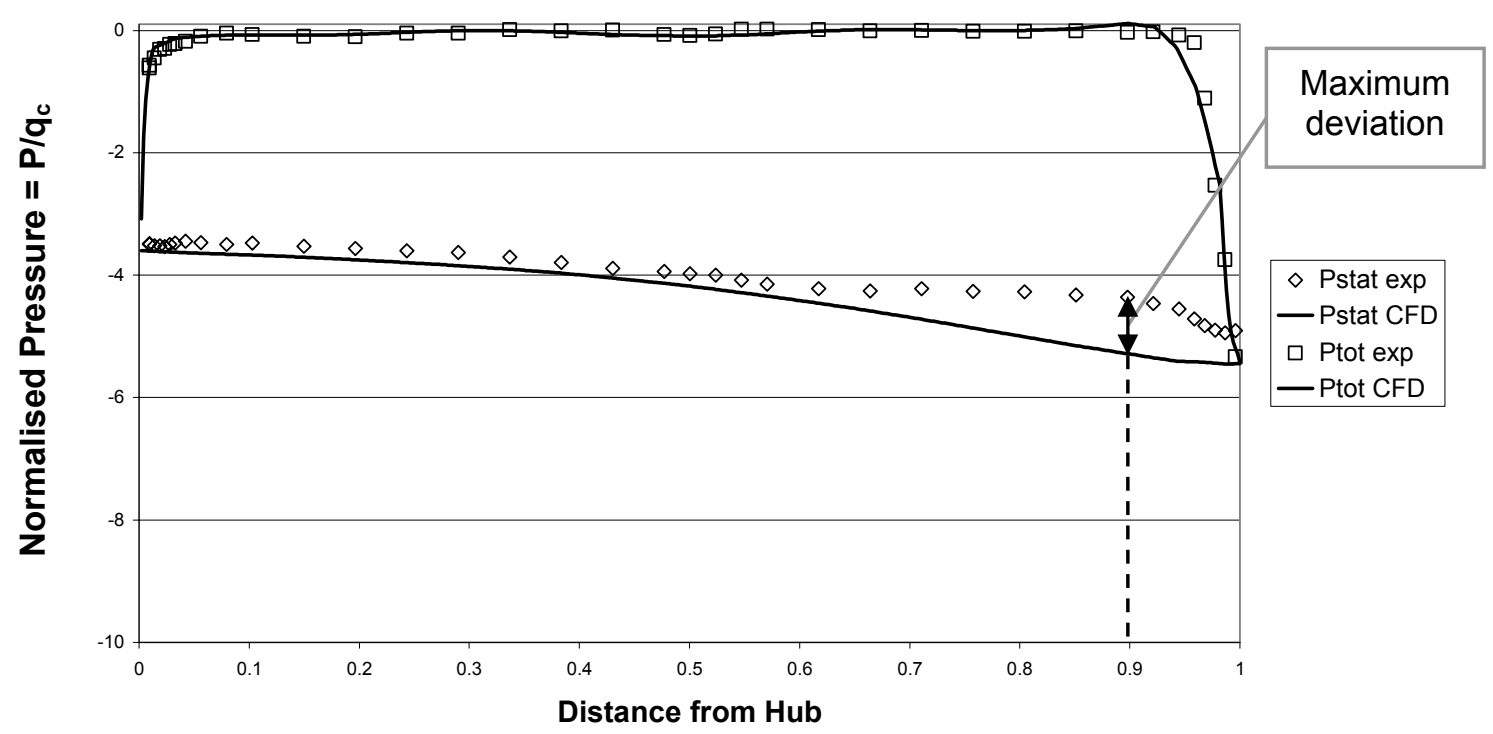

Figure 4-14 - Row 7 top pressure; $22.5^{\circ} 260 \mathrm{~mm}$

Figure 4-12, Figure 4-13 and Figure 4-14 show that there is good agreement of the boundary layers (simulated by the total pressure) on the inner wall but not always near the outer wall. The outer wall is over predicted by the CFD (also seen in Figure 4-15), because the flow is simulated as turbulent throughout the entire flow regime. The five-hole probe would measure a slightly smaller boundary layer since there is a transitional boundary layer in this region instead of a fully turbulent one as simulated. Leakage occurred around the sleeve from outside in the cases where a thicker boundary layer was measured.

The measurement of the middle-row outer boundary layer is relatively inaccurate in the traverse of Figure 4-13, when considering that the agreement for the top and middle plane boundary layers is generally good (as seen in Appendix B:). However, the general trend was predicted satisfactory for the total pressure.

All of the total pressures were measured within the expected errors mentioned in \$4.2.2. To determine the average pressure loss through the transition a discretised equation in a similar form as equation (4-20) and (4-21) is used. The total pressure is calculated using the mass flow average. Since the flow is incompressible, the flow 
rate average can be used, therefore the average total pressure loss is calculated as follows.

$$
\bar{P}_{t o t}=\frac{\int P_{t o t} \cdot V \cdot d A}{\int V \cdot d A}
$$

From equation (4-16), (4-20) and (4-21) the total pressure of equation (4-22) can be rewritten as

$$
\bar{P}_{t o t}=\frac{1}{8 \cdot Q} \sum_{j=1}^{r o w-1} \sum_{i=1}^{n-1}\left(P_{t o t, i+1, j}+P_{t o t, i, j}\right)\left(V_{\perp, i+1, j}+V_{\perp, i, j}\right)\left(S_{i+1, j}^{2}-S_{i, j}^{2}\right) \cdot \varphi \cdot \operatorname{Cos} \theta
$$

The comparisons of the results are shown in the table below. The configuration symbols are described in the nomenclature.

Table 4-3 - Transition section total pressure loss

\begin{tabular}{|c|c|c|c|c|c|}
\hline \multirow{2}{*}{ Configuration } & \multicolumn{2}{|c|}{ CFD } & \multicolumn{2}{c|}{ EFD } & \multirow{2}{*}{} \\
\cline { 2 - 6 } & $\mathrm{P}_{\text {tot }}[\mathrm{Pa}]$ & $\mathrm{C}_{\mathrm{L}}$ & $\mathrm{P}_{\text {tot }}[\mathrm{Pa}]$ & $\mathrm{C}_{\mathrm{L}}$ & $\mathrm{C}_{\mathrm{L}}$ Error [\%] \\
\hline $\mathbf{A}$ & -3.89 & 0.098 & -5.42 & 0.138 & -29 \\
\hline $\mathbf{B}$ & -4.62 & 0.117 & -3.69 & 0.096 & +22 \\
\hline $\mathbf{C}$ & -6.00 & 0.151 & -5.63 & 0.144 & +4.9 \\
\hline $\mathbf{D}$ & -7.56 & 0.191 & -7.14 & 0.195 & -1.2 \\
\hline
\end{tabular}

The results in the table above are somewhat misleading. Figure 4-15 shows a close-up comparison of the total pressures for one of the traverses in the top plane for case A. The smoothed experimental line follows the trend of the CFD accurately for most of the traverse, but is clearly offset by about the variance of a transducer (indicated by the error markers). Since the transducers are calibrated, they can have an offset error with the magnitude of the variance. For instance the EFD result for setup A, has an error of $-29 \%$ in comparison with the CFD result. If the variance of a pressure transducer, namely $1.725 \mathrm{~Pa}$, is now added to the EFD $\mathrm{P}_{\text {tot }}$ the new results become: $\mathrm{P}_{\text {tot }}=-3.70 \mathrm{~Pa}$ and hence $\mathrm{C}_{\mathrm{L}}=0.095$. This changes the error to $+3.7 \%$. The only conclusion is that the variance has become a significant factor as predicted in $§ 4.2 .2$. Not much more than the order of magnitude can be predicted with the EFD. 
The order of magnitude of the losses can be disclosed from the CFD analyses in spite of the errors in Table 4-3. The pressure distribution of the CFD is fairly consistent with the fitted curves through the EFD results. Furthermore, we expect that a few places along the flow passage are insignificantly affected by walls, thereby giving almost no total pressure loss in those regions. The CFD does indicate such regions shown in Figure 4-15. However the CFD results seem to be optimistic. Numerical dispersion due to the higher order analysis caused the total pressure to become positive in a few regions (indicated in Figure 4-15 and discussed in §3.3.4). This leads to the conclusion that the CFD gives a slightly optimistic result when calculating the average total pressure loss through the section.

Ptotal Comparison: Row 2; $22.5^{\circ}$ IGV angle and $320 \mathrm{~mm}$ Collector

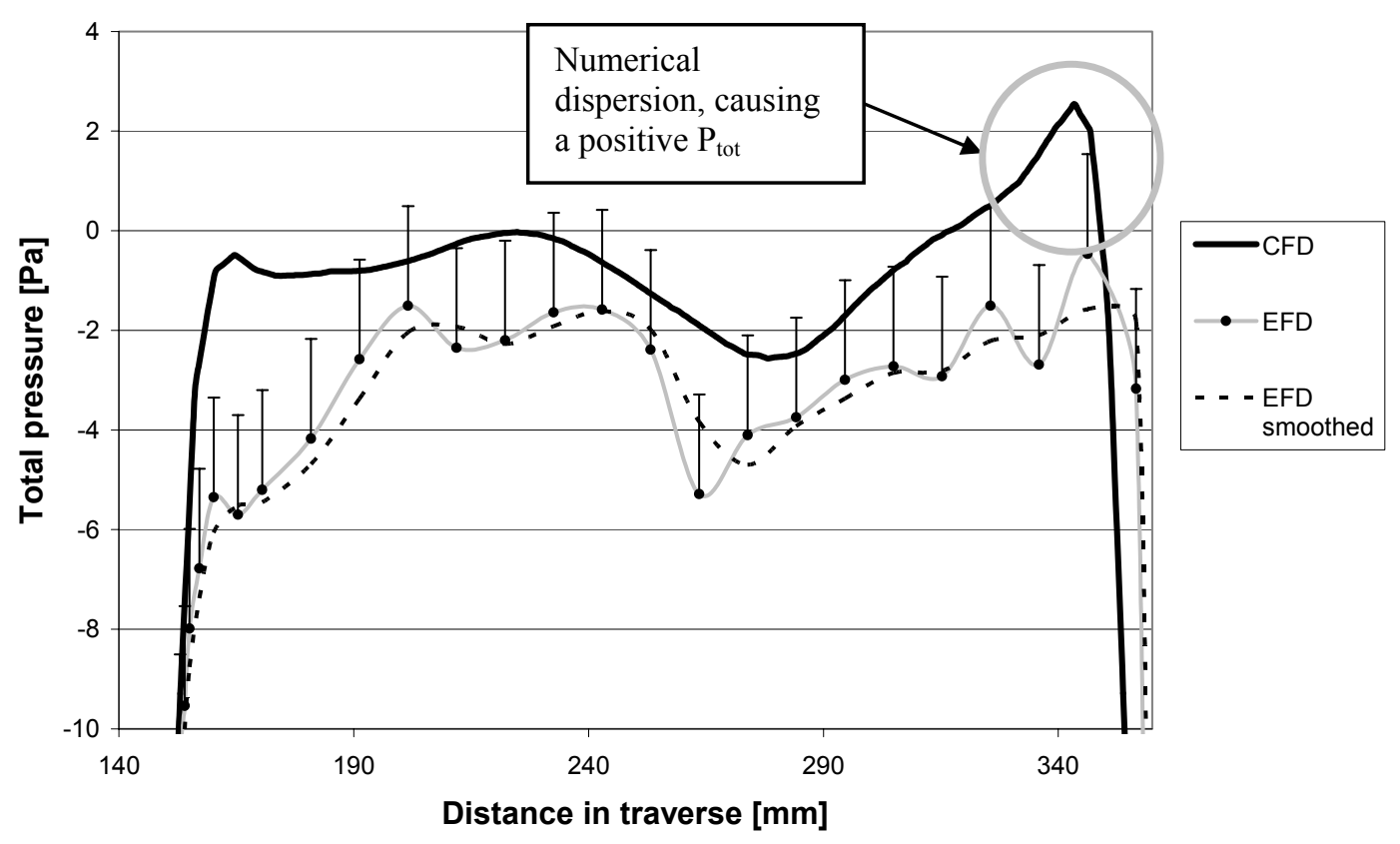

Figure 4-15 - CFD and EFD $P_{\text {total }}$ comparison

The static pressure is predicted accurately for all the bottom planes (Figure 4-12). However the middle- and top planes over predict the magnitude of the static pressure from $60-99 \%$ of the traverse distance (seen in Figure 4-13 and Figure 4-14). The static pressure seems to have to follow the trend of the CFD, but the EFD measures a type of wake that returns to the simulated value when approaching the outer wall. This is confirmed by the correlation on the outer wall discussed in $\S 4.3$. 
The static pressure discrepancy near the outer wall (indicated in Figure 4-13 and Figure 4-14) could be explained by Taylor-Görtler vortices. When flow is laminar or transitional, centrifugal instabilities can cause toroidal vortices to occur (White, 1991). CFD software is still unable to simulate transitional flow accurately and therefore it does not simulate specific effects of transitional flow (Oberkampf and Blottner, 1998). This is challenging since it is shown in $\S$ A.1 that the flow is indeed transitional through the transition section of the experimental rig. Vortices in annuli were investigated by Hall (1982) for fully developed or boundary layer flows. Later on the nonlinear interaction of the Tolmien Schlichting waves and Taylor-Görtler vortices were investigated by Bennet et al. (1991). Both predicted whether the vortices would occur using a parameter known as the Taylor number.

Since the Taylor number is a value that describes chaotic behaviour in flow similar to the Reynolds number, there are many definitions of it. In all of these definitions the inner wall rotational speed, gap size and dynamic viscosity is required. The definition for the Taylor number that will be used for this thesis is the one of Brenner (2000)

$$
T a=\frac{4 \cdot d^{4} \cdot \Omega_{\text {inner }} \cdot A}{v^{2}}
$$

where $\boldsymbol{d}$ is the gap width, $\boldsymbol{\Omega}_{\text {inner }}$ the inner wall rotational velocity, $\boldsymbol{A}$ the gap area and $\boldsymbol{v}$ the dynamic viscosity. The critical Taylor number is

$$
T a_{c r} \approx \frac{3430}{1+\mu} \approx 3430
$$

Since the transition section of the rig has a stationary inner wall, a virtual inner wall was chosen away from the outer wall to perform the calculations. The Taylor numbers for the two deviant cases discussed in this chapter are shown in Figure 4-16. The Taylor number reached the critical value at a distance of $\approx 11 \%$ from the outer wall. This is almost exactly where the discrepancy observed in Figure 4-13 and Figure 4-14 is at its maximum. Further away from the outer wall, the Taylor number quickly increases to orders of magnitude larger than the critical Taylor number. In those regions, the CFD predicts the flow behaviour more accurately. 


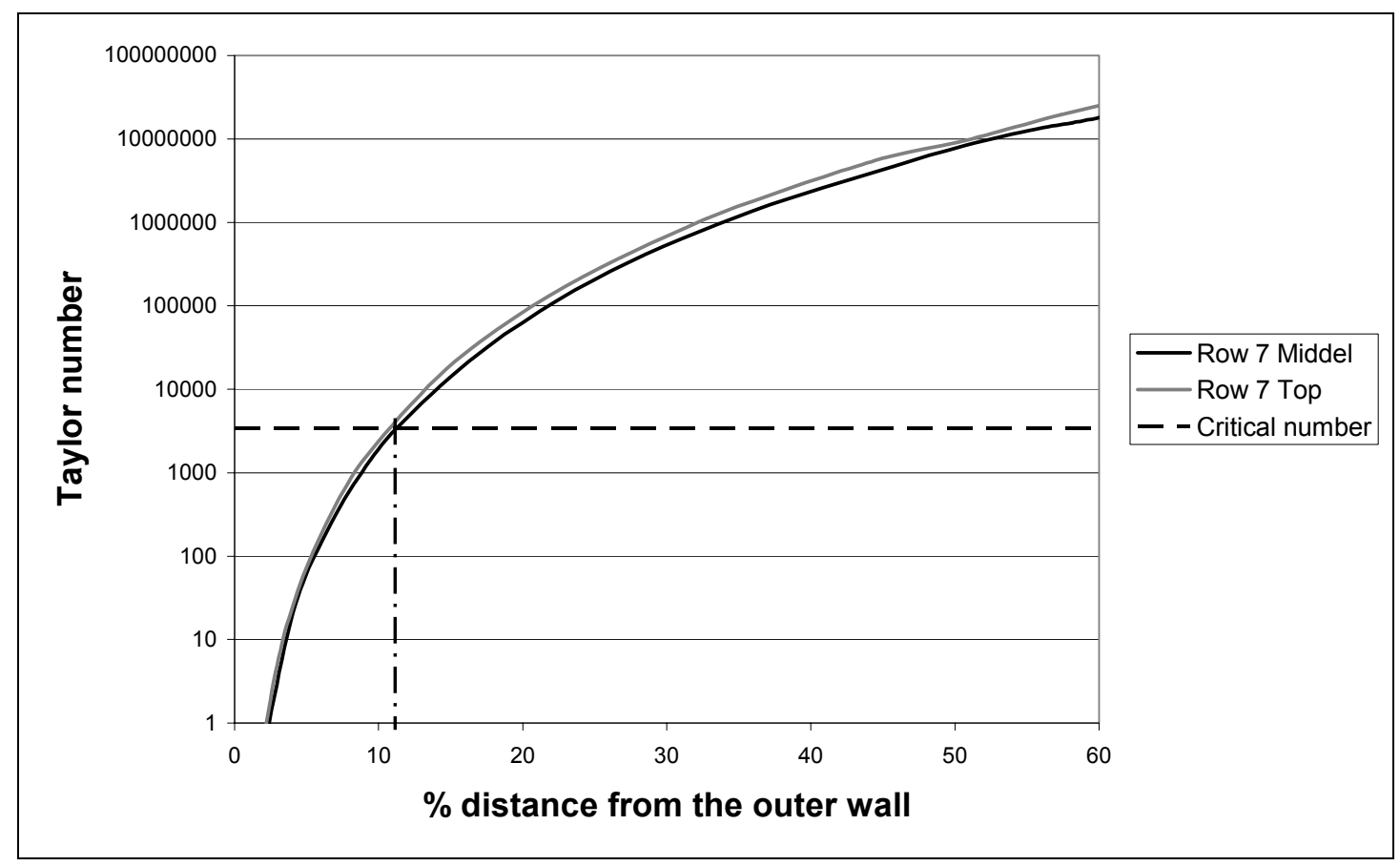

Figure 4-16 - Taylor numbers of the experimental rig

Hall (1982) observed that the vortices were away from the wall in the free stream at high wave numbers. In this project, it seems, the same behaviour is being observed. The transitional effects were studied by Bennet et al. (1991) and it was found that the vortices occur more readily under transitional conditions. Gannon (2002) designed the entire experimental rig to let the flow regime be predominantly laminar throughout it, but as shown in $\S$ A.1, the flow becomes turbulent at the outer wall near to where the experimental readings were taken. Therefore there is a possibility that the regions away from the wall are still laminar or transitional.

From the behaviour observed in visual confirmation $(\$ 2.5 .3)$, the results seen and the descriptions of Hall (1982), it seems to be an obvious conclusion that the discrepancies are caused predominantly due to Taylor-Görtler vortices. This is a laminar-to-turbulent transition effect that the CFD software cannot simulate and is also beyond the scope of this project.

\subsubsection{FLOW ANGLES}

The three figures that follow below indicate the flow angle predictions and measurements. 


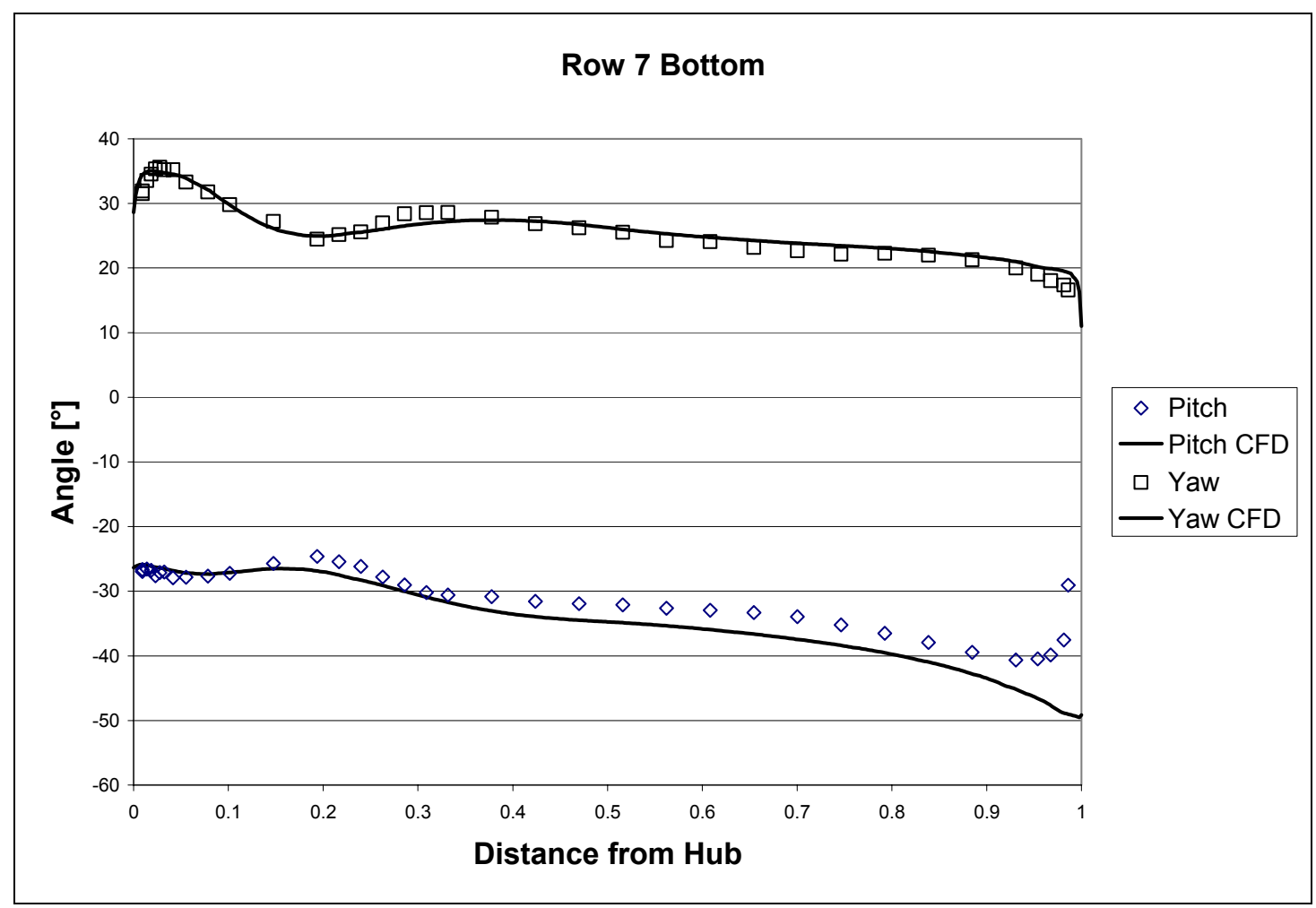

Figure 4-17 - Row 7 bottom angles; $22.5^{\circ} 260 \mathrm{~mm}$

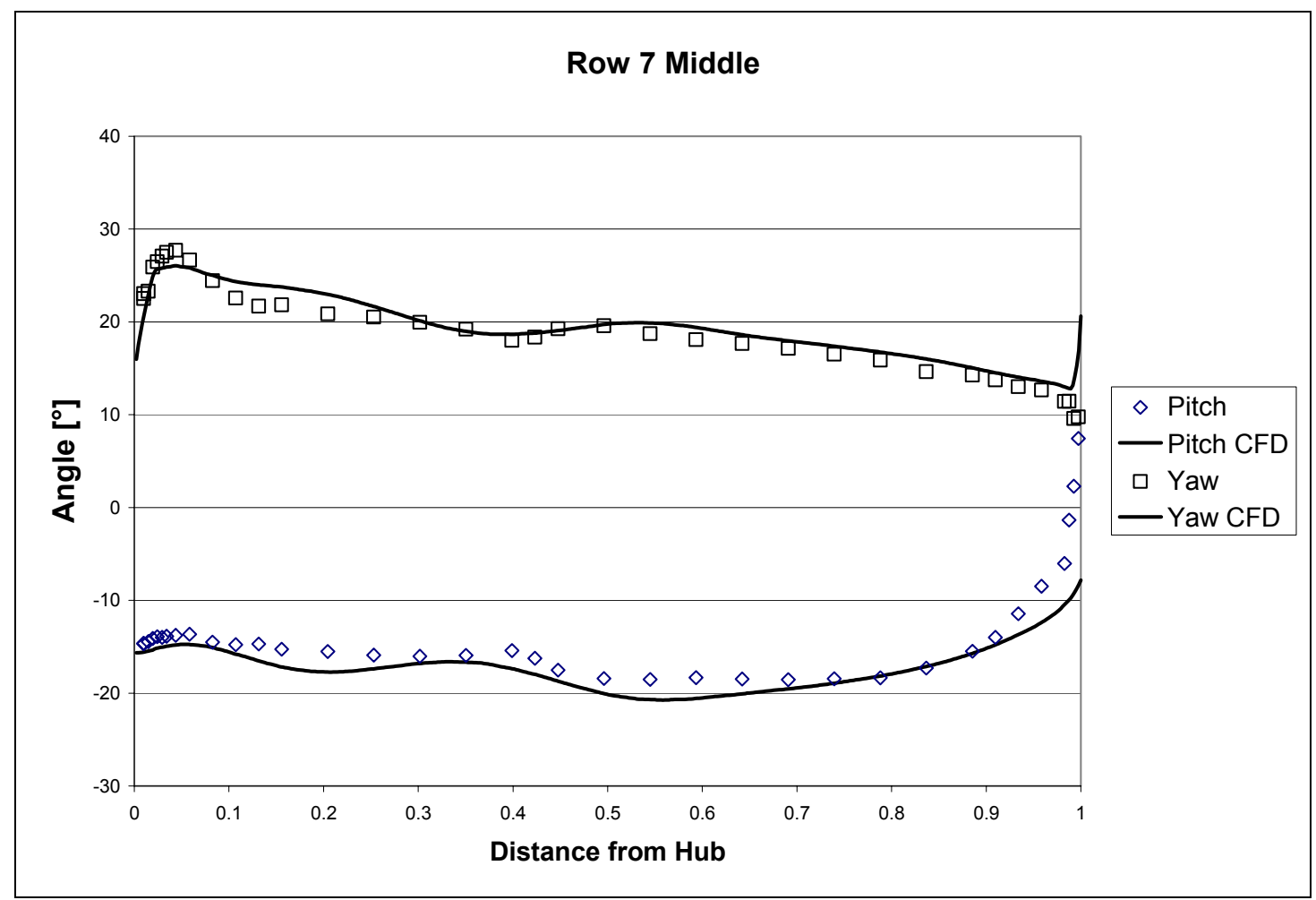

Figure 4-18 - Row 7 middle angles; $22.5^{\circ} 260 \mathrm{~mm}$ 


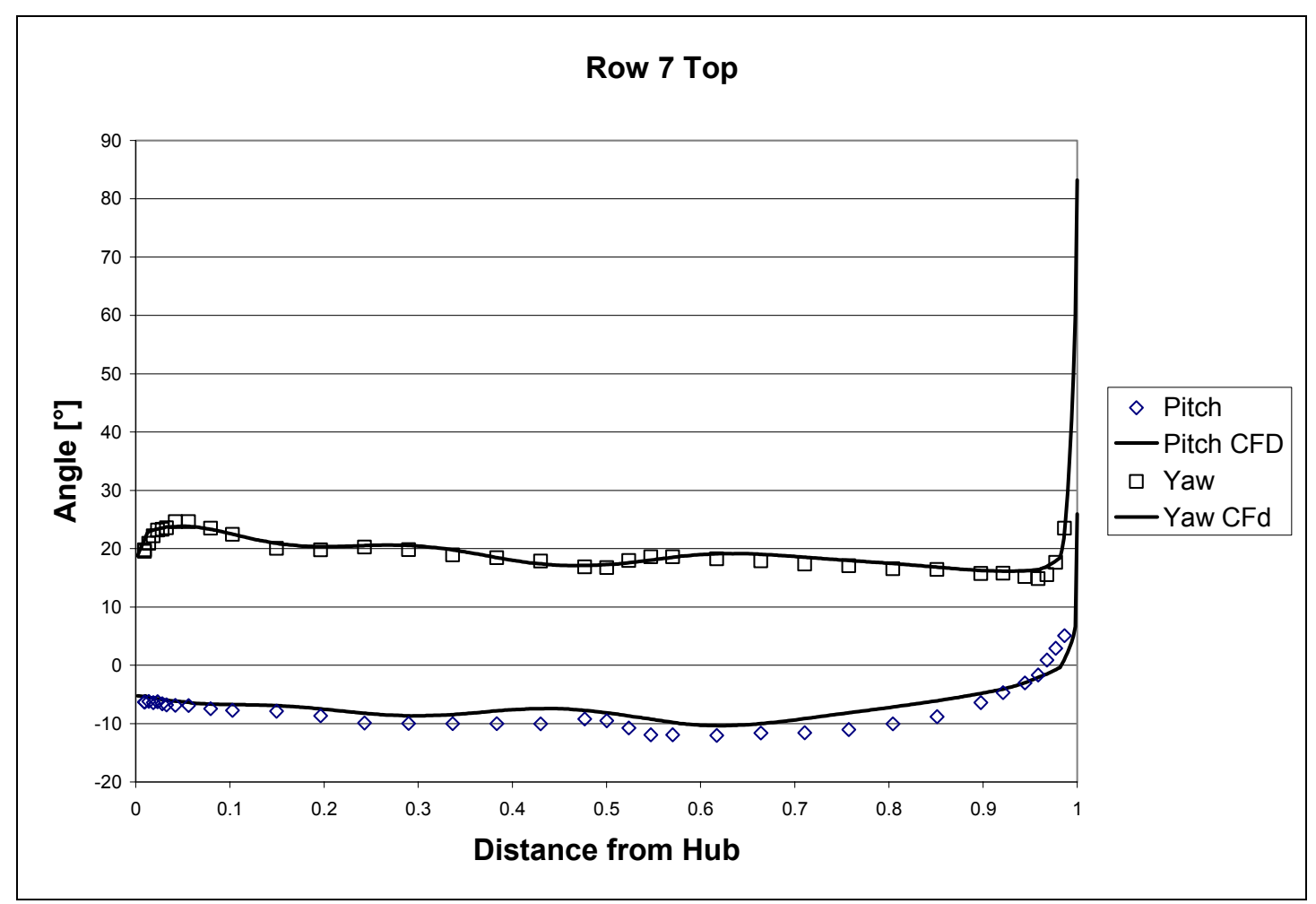

Figure 4-19 - Row 7 top angles; $22.5^{\circ} 260 \mathrm{~mm}$

Figure 4-17, Figure 4-18 and Figure 4-19 above indicate that the angle predictions are within $5^{\circ}$ to the measured values and within $2^{\circ}$ on average. The yaw angle results are predicted more accurately than the pitch angles, averaging on about $1^{\circ}$ errors for most cases. As explained in $\S 4.2 .2$, these predictions are as accurate as could be achieved realistically with this project.

The wall- and hole effects on the probe have made the erroneous angle predictions near the outer wall especially for the pitch angles. The pitch angles for row 4 are generally not predicted well near the outer wall. The magnitude of the wake form is under predicted by the CFD by as much as $10^{\circ}$. However for the purpose of the design of the turbine blades, the yaw angles are of greater importance and these have been calculated satisfactorily.

The average turning angles for the configurations defined in the nomenclature are:
$\mathrm{A}-16.05^{\circ}$
$\mathrm{B}-23.21^{\circ}$
$\mathrm{C}-19.15^{\circ}$
D $-27.57^{\circ}$ 


\subsection{Full Scale Performance Prediction}

The full scale results were generated by using the same geometry of the experimental rig, and rescaling it. The simulation was done with no thermal models and used the conditions described in Von Backström, Kirstein and Pillay (2003) where they were applicable. The lowered deck was simulated by extending the vertical section above the outer wall curve upward to meet the diffuser (indicated in Figure 4-20). The IGV stagger angle was changed by pivoting the IGVs around its tail tip. By comparing Figure 4-20 with Figure 4-21, it can be seen how the IGV stagger angle and the collector height changes with the varying configurations. Both of these figures use the IGV and chimney entrance geometry described by Gannon (2002).

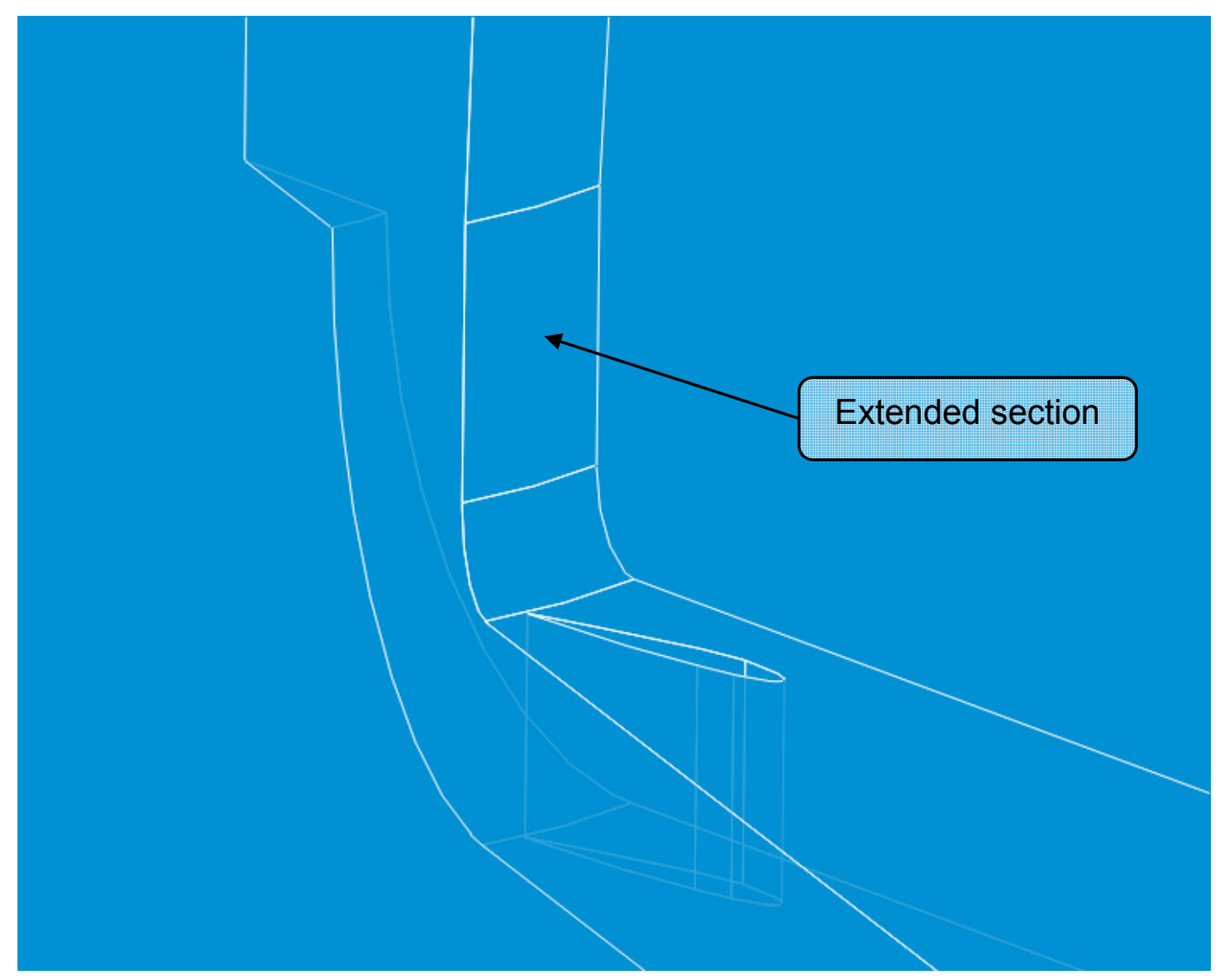

Figure 4-20 - Full scale modification to a low deck

Table 4-4 and Table 4-5 below contain the numerical results that were extracted from the CFD simulations. The objective was to determine the loss coefficient through the section and the average turning angle at the exit (just below the turbine). The loss 
coefficients were determined from the total pressure loss through the section from the start of the IGVs to just below the turbine, and normalised by the dynamic pressure of the through-flow of a nominal chimney diameter.

The reason for taking an average turning angle is for simplicity in illustrating the results. Besides the exit turning angle through the transition section is fairly constant in all experimental cases (see results for top traverses in $\S$ B. 1 to $\S$ B.4).

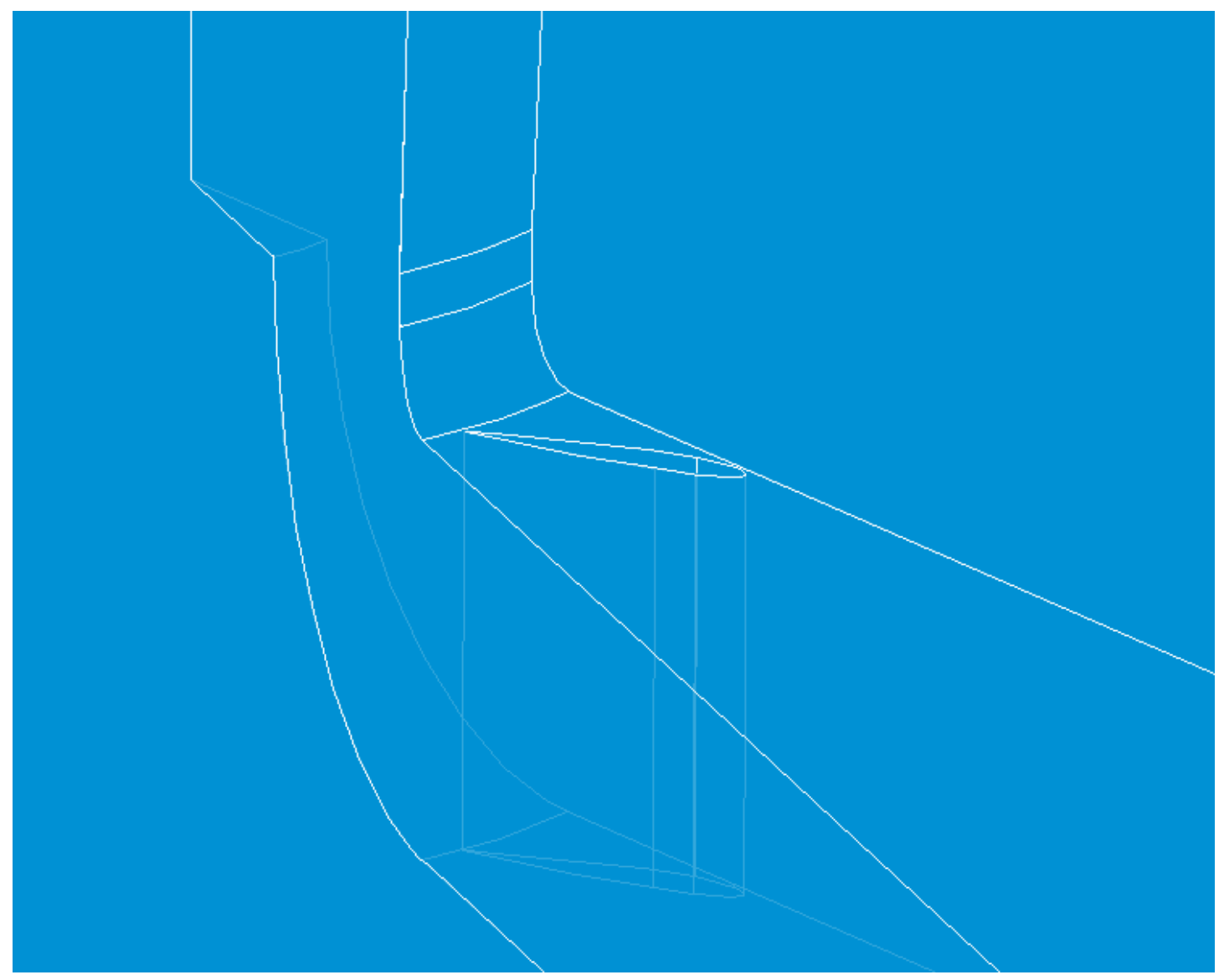

Figure 4-21 - Full scale modification to a high deck

Table 4-4 - Full scale plant turning angles $\left[{ }^{\circ}\right]$

\begin{tabular}{|c|c|c|c|c|c|c|}
\hline \multirow{2}{*}{$\begin{array}{c}\text { Deck } \\
\text { height }\end{array}$} & \multicolumn{7}{|c|}{ IGV stagger angle $\left[^{\circ}\right]$} \\
\cline { 2 - 7 } & $\mathbf{0}^{\circ}$ & $\mathbf{1 0}^{\circ}$ & $\mathbf{2 0}^{\circ}$ & $\mathbf{2 5}^{\circ}$ & $\mathbf{3 0}^{\circ}$ & $\mathbf{3 5}^{\circ}$ \\
\hline $\mathbf{0 . 2 0 D}$ & 0.44 & 12.18 & 23.47 & 28.98 & 34.39 & 39.76 \\
\hline $\mathbf{0 . 2 5 D}$ & 0.44 & 9.74 & 19.13 & 23.85 & 28.63 & 33.46 \\
\hline $\mathbf{0 . 3 0 D}$ & 0.37 & 8.10 & 16.08 & 20.23 & 24.47 & 28.81 \\
\hline $\mathbf{0 . 3 5 D}$ & 0.30 & 6.31 & 13.84 & 17.50 & 21.30 & 25.26 \\
\hline $\mathbf{0 . 4 0 D}$ & 0.30 & 6.31 & 12.67 & 16.07 & 19.59 & 23.32 \\
\hline
\end{tabular}


Table 4-5 - Full scale plant loss coefficient of transition section

\begin{tabular}{|c|c|c|c|c|c|c|}
\hline \multirow{2}{*}{$\begin{array}{c}\text { Deck } \\
\text { height }\end{array}$} & \multicolumn{7}{|c|}{ IGV stagger angle $\left[{ }^{\circ}\right]$} \\
\cline { 2 - 7 } & $\mathbf{0}^{\circ}$ & $\mathbf{1 0}^{\circ}$ & $\mathbf{2 0}^{\circ}$ & $\mathbf{2 5}^{\circ}$ & $\mathbf{3 0}^{\circ}$ & $\mathbf{3 5}^{\circ}$ \\
\hline $\mathbf{0 . 2 0 D}$ & 0.0630 & 0.0668 & 0.0953 & 0.1228 & 0.1625 & 0.2257 \\
\hline $\mathbf{0 . 2 5 D}$ & 0.0434 & 0.0534 & 0.0722 & 0.0885 & 0.1189 & 0.1539 \\
\hline $\mathbf{0 . 3 0 D}$ & 0.0454 & 0.0467 & 0.0578 & 0.0702 & 0.0923 & 0.1067 \\
\hline $\mathbf{0 . 3 5 D}$ & 0.0427 & 0.0437 & 0.0524 & 0.0626 & 0.0778 & 0.0835 \\
\hline $\mathbf{0 . 4 0 D}$ & 0.0363 & 0.0368 & 0.0424 & 0.0483 & 0.0579 & 0.0661 \\
\hline
\end{tabular}

The flow angle into the turbine is a function of the deflection angle through the IGV cascade, modified by the effect of the area ratio on the through-flow component and the conservation of angular momentum on the tangential component. Consequently the radial position of the IGV's $25 \%$ chord length, the flow area at that point, the flow area at the turbine and the radius of an equivalent streamline at the turbine contributes to the final result. It was however found that the flow angle, $\boldsymbol{\theta}$, into the turbine could be described by the following empirical formula.

$$
\tan (\theta) \approx 0.238 \frac{\tan (\beta)}{H}
$$

Where $\boldsymbol{\beta}$ is the stagger angle of the IGV cascade and $\mathbf{H}$ the collector-height-tochimney-diameter ratio, $\mathrm{h} / \mathrm{D}$. The magnitude of the average error when compared to the simulated data is $\approx 1.6 \%$. The exit angle of the IGV cascade is almost the same as the IGV stagger angle ( $\S \mathrm{A} .2)$; therefore $\boldsymbol{\beta}$ in equation (4-26) can be substituted with the IGV stagger angle, $\varphi$, So that the flow angle out of the IGV cascade is used instead. This yields:

$$
\theta \approx \tan ^{-1}\left(0.238 \frac{\tan (\phi)}{H}\right)
$$

The comparison between equation (4-26) and the results that were simulated with CFD is shown in Figure 4-22 below. 


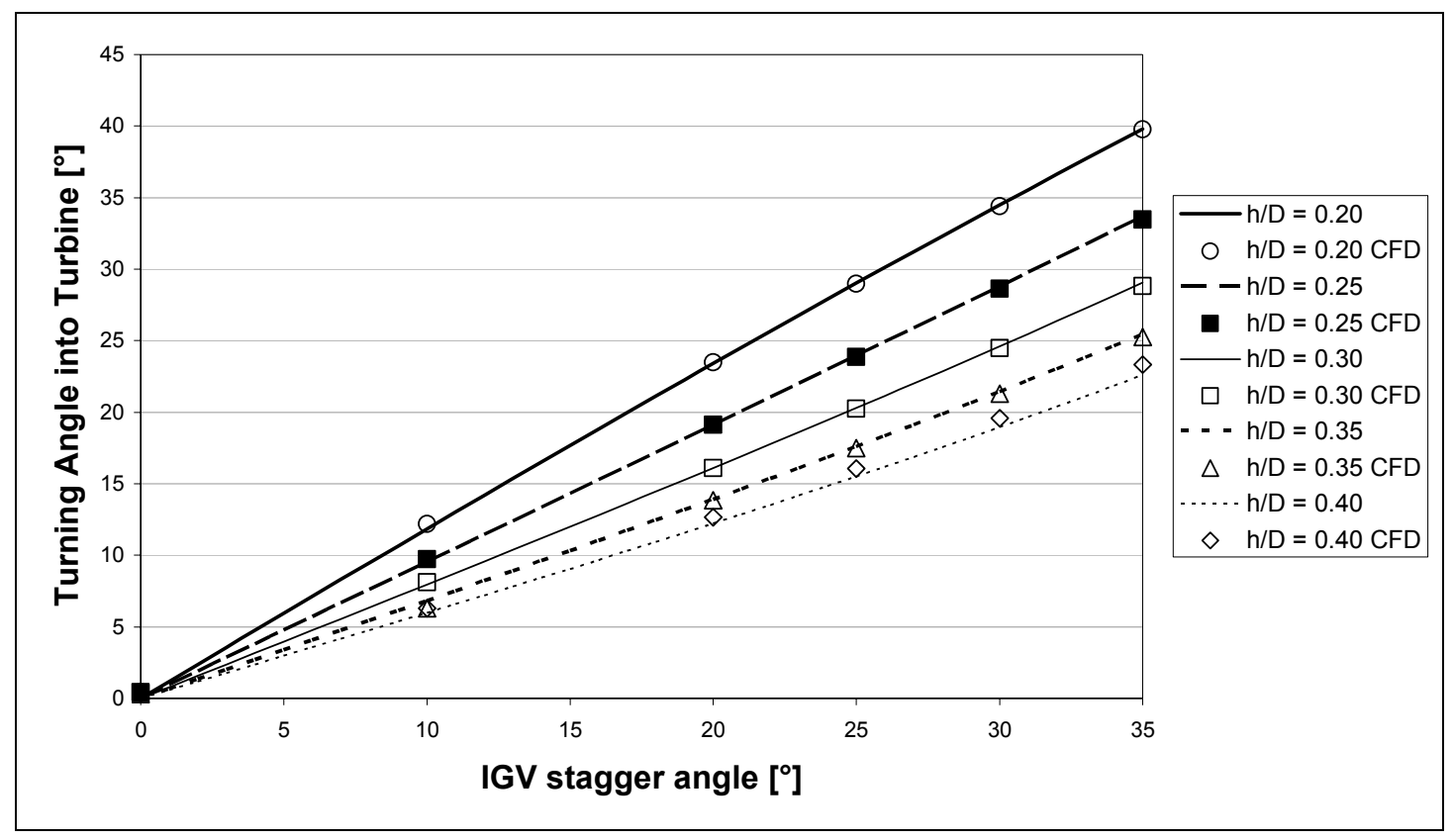

Figure 4-22 - Full scale performance: turning angle

The loss coefficient presents a more difficult task in determining what influences the losses. The first assumption is that loss is proportional to the total velocity squared. The total velocity can be made up by the vector sum of the through-flow velocity and the rotational velocity. Furthermore the IGVs will contribute to the loss with a component proportional to the square of the total velocity through the cascade. Therefore the three primary loss mechanisms can be summarised as:

- The wall friction of the non-rotational flow component in the transition section, which is a constant.

- The wall friction of the rotational flow component into the chimney, which is proportional to $\tan (\theta)$

- The IGV profile loss, which is proportional to the square of the total velocity through the cascade, which is proportional to $\sec (\varphi) / \mathrm{H}$

This yields a loss function in the form of

$$
C_{L}=c_{1}^{2}+c_{2}^{2}\left(\frac{\sec \phi}{H}\right)^{2}+c_{3}^{2} \tan ^{2} \theta
$$

Using this loss function and that assuming that $\boldsymbol{\beta} \approx \boldsymbol{\varphi}$ (see $\S \mathrm{A} .2$ for explanation), the loss is $f(\boldsymbol{\beta}, \mathbf{H})$, which yields 


$$
C_{L} \approx 0.0292+0.00114\left(\frac{\sec \beta}{H}\right)^{2}+0.190 \tan ^{2}(\theta)
$$

For the loss coefficient through the transition section, where $\boldsymbol{\beta} \varepsilon\left[0^{\circ}, \mathbf{3 5}^{\circ}\right]$ is the IGV stagger angle and $\mathbf{H} \boldsymbol{\varepsilon}[\mathbf{0 . 2}, \mathbf{0 . 4}]$ the collector roof height as a fraction of the chimney diameter. The functions from equation (4-29) come from the relations between the dynamic pressures. Equation ( 4-29) is deviant from the simulated values by an average of $4.7 \%$ and a maximum of $10.0 \%$. This model makes it possible to correlate the friction term with the Reynolds number and possibly also the effect of transition length and turbine-to-chimney diameter ratio. This is however beyond the scope of this project.

The comparison between equation (4-29) and the results that were simulated with CFD is shown in Figure 4-23 below.

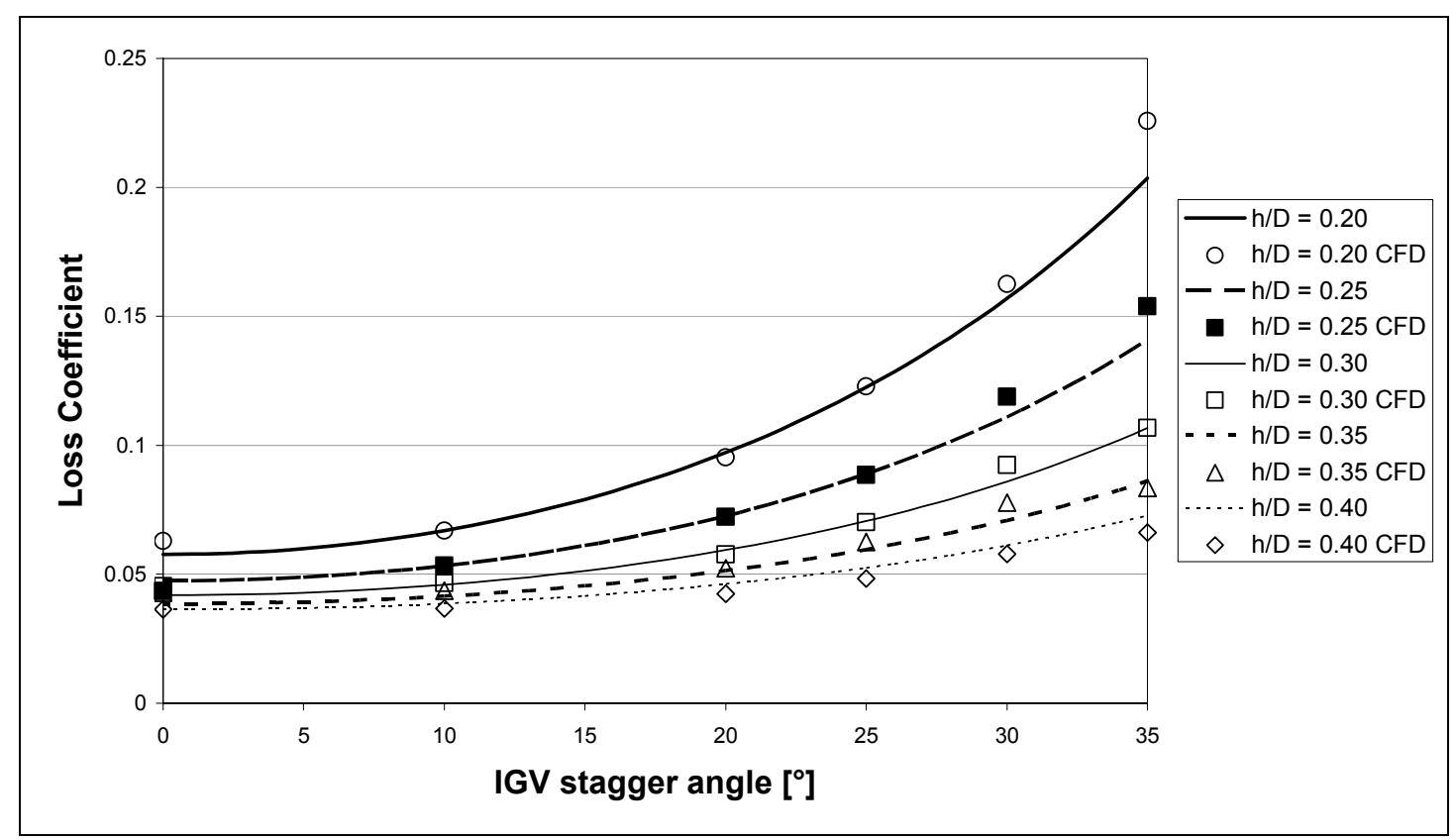

Figure 4-23 - Full scale performance: transition section loss

Gannon (2002) estimated the transition loss coefficient of the scale model to be $\approx 0.163$ when based on the dynamic pressure of a nominal chimney diameter. Von Backström, Kirstein and Pillay (2003) refined Gannon's (2002) estimate to $\approx 0.161$ by taking the collector inlet loss coefficient into account. Although these losses were 
determined for the SCPP scale model, it was assumed that they were also applicable to the full scale geometry plant. The loss coefficient for configuration $\mathrm{A}$ in the experimental rig was determined to be $\approx 0.098$, which is lower than previous estimations. According to CFD analyses for the full scale geometry, the loss coefficient for configuration $\mathrm{A}$ is instead closer to $\approx 0.056$. Hence it should be noted that smaller scale model predicts higher losses than the larger scales.

The losses are lower with larger scales, because for transitional flow with smooth surfaces, the friction factor decreases as the Reynolds number is increased. This is seen in the Moody chart for pipe friction (White, 1999). By comparing the Reynolds numbers based on diameter in the chimneys, it can be seen that the Reynolds number of the full scale plant is in the order of $10^{8}$ and the experimental rig in the order of $10^{5}$, which is a difference of three orders of magnitude. In the Moody chart an almost smooth pipe $(\varepsilon / \mathrm{d}=0.00001)$ has a friction factor ratio between $\mathrm{Re}=10^{5}$ and $\mathrm{Re}=10^{8}$ of $\approx 1.9$. The ratio between the experimental- and the full scale loss coefficient is $\approx 1.8$. This is close enough to validate the assumption of the Reynolds number effect.

The loss coefficients and average turning angles of the four configurations for the full scale SCPP are summarised in the table below. The configurations are described in the nomenclature.

Table 4-6 - CFD results for a full scale geometry

\begin{tabular}{|c|c|c|}
\hline \multirow{2}{*}{ Configuration } & $\begin{array}{c}\text { Total Pressure } \\
\text { Loss }\end{array}$ & $\begin{array}{c}\text { Average Exit } \\
\text { Turning Angle } \boldsymbol{\theta}\end{array}$ \\
\cline { 2 - 3 } & $\mathrm{C}_{\mathrm{L}}$ & $\left.{ }^{\circ}\right]$ \\
\hline A & 0.0558 & 15.5 \\
\hline B & 0.0771 & 22.9 \\
\hline C & 0.0676 & 18.9 \\
\hline D & 0.1060 & 26.7 \\
\hline
\end{tabular}




\section{CHAPTER 5: CONCLUSIONS AND RECOMMENDATIONS}

The main aim of this project was to determine the performance of the collector-tochimney transition section of a SCPP. The project also established more tools for modelling the SCPP.

\subsection{OutComes}

\section{Transition Section Performance}

The performance of the experimental transition section was calculated and measured as accurately as the CFD software and experimental equipment allowed. The rig's performances for the different configurations are summarised in Table 5-1. The configurations are described in the nomenclature.

Table 5-1 - Experimental rig transition section performance

\begin{tabular}{|c|c|c|c|}
\hline \multirow{2}{*}{ CONFIGURATION } & \multicolumn{2}{|c|}{ Total Pressure Loss } & $\begin{array}{c}\text { Average Exit } \\
\text { Turning Angle } \boldsymbol{\theta}\end{array}$ \\
\cline { 2 - 4 } & $\mathrm{P}_{\text {tot }}[\mathrm{Pa}]$ & $\mathrm{C}_{\mathrm{L}}$ & $\left.{ }^{\circ}\right]$ \\
\hline A & -3.89 & 0.098 & 16.05 \\
\hline B & -4.62 & 0.117 & 23.21 \\
\hline C & -6.00 & 0.151 & 19.15 \\
\hline D & -7.56 & 0.191 & 27.57 \\
\hline
\end{tabular}

The investigation was extended to a proposed full scale geometry and its performance obtained. Previous estimates for the loss coefficient have been $\approx 0.25$ and $\approx 0.161$ for configuration A. This thesis however shows that the loss coefficient is closer to $\approx 0.056$.

The full scale plant performance is summarised in the two equations below.

$$
\tan (\theta) \approx 0.238 \frac{\tan (\beta)}{H}
$$


where $\boldsymbol{\theta}$ is the average turning angle into the turbine, and

$$
C_{L} \approx 0.0292+0.00114\left(\frac{\sec \beta}{H}\right)^{2}+0.190 \tan ^{2}(\theta)
$$

for the loss coefficient through the transition section, where $\boldsymbol{\beta} \varepsilon\left[0^{\circ}, \mathbf{3 5}^{\circ}\right]$ is the IGV stagger angle and $\boldsymbol{H} \varepsilon[\mathbf{0 . 2}, \mathbf{0 . 4}]$ the collector roof height as a fraction of the nominal chimney diameter.

\section{Numerical Modelling of Flow Behaviour}

With the exception of not being able to simulate the Taylor-Görtler vortices generated near the outer wall, the CFD software simulated the flow behaviour satisfactorily.

\section{Experimental Measurement of Flow Behaviour}

The magnitude of the total pressure loss through the transition section was too low for the equipment to be able to measure with a high degree of accuracy. The experimental results for the total pressure therefore merely served to confirm that the CFD simulated the position and order of magnitude of the IGV wakes losses correctly. The rest of the flow behaviour was measured satisfactorily.

\section{Strength Alterations}

It was found in this project (in $\S \mathrm{A} .3$ ) that by increasing the IGVs stagger angle beyond $10^{\circ}$ the torsional stiffness ratio of the chimney support is increased. Increasing the stagger angle from $22.5^{\circ}$ to $31.5^{\circ}$ increases the torsional stiffness by $72 \%$.

\subsection{RECOMMENDATIONS}

- This project is to lay the foundation for the work that follows. It is suggested that further investigations are made with the turbine included. This would require further investigation in

- Prediction of the turning angles into and out of the turbine

- The performance correlation of the turbine in EFD and CFD by the measurement of the power maps for the four IGV configurations combined with turbine blade angles. 
- To make use of the power maps and turbine, varispeed drives are required. Therefore a way to shield the AT transducers from the noise of the varispeed drives should be implemented.

- Investigation into better grid generation software to reduce the required number of grid points through the transition section.

- The five-hole probe could obtain more accurate readings by installing it into a better bracket.

- The calibration maps for the five-hole probe could be refined and extended with the calibration program that was written. Statistics recommend that at least 30 samples should be taken at each point, but more than 50 for accurate results.

- The five-hole probe should not be used at low Reynolds numbers, since the readings are inconsistent. It would not even be worthwhile to try and calibrate it for low Reynolds numbers for this very reason. The rig could be upgraded with a stronger motor to increase the through-flow velocity. 


\section{REFERENCES}

Aungier (2000): Axial flow compressors: A strategy for aerodynamic design and analysis. ASME Press, New York, 2003

Brenner M., http://www.deas.harvard.edu/brenner/taylor/handouts/taylor_couette/node3.html. Last updated on Sun Feb 6 15:47:39 EST 2000, last accessed 2004-08-21. Contact details: brenner@math.mit.edu

Bennet J., Hall P. and Smith F.T., The strong nonlinear interaction of Tolmien Schlichting waves and Taylor-Görtler vortices in curved channel flow, Journal of Fluid Mechanics, 1991, Vol. 223, pp. 475-495

Blanchard B. S. and Fabrycky W. J. (1998), Systems engineering and analysis, third edition, Prentice Hall, Upper Saddle River

Chuang C. C. and Chieng C. C., Comparative study of higher order turbulence models for compressible separated flows, AAIA journal, Vol.32, No.8, 1994, pp. 1740-1743

Crouch J. D., Crouch I. W. M. and Ng L. L., Transition prediction for threedimensional boundary layers in computational fluid dynamics applications, AAIA journal, Vol.40, No.8, August 2002, pp. 1536-1541.

De Jager, L.C. (2000), The Design and Modelling of a Solar Chimney Turbine Inlet, Department of Mechanical Engineering, University of Stellenbosch

Demmel J.W. (1997), Applied Numerical Linear Algebra, Siam: Society of Industrial and Applied Mathematics.

Gannon, A.J. (2002), Solar Chimney Turbine Performance, Ph.D. Thesis, Department Mechanical Engineering, University of Stellenbosch, South Africa

Gannon A. J. and Von Backström T. W., Solar Chimney Turbine Performance, ASME Journal of Solar Energy Engineering, Vol. 125, No. 1, pp. 101-106, February 2003

Gannon A. J. and Von Backström T. W., Solar Chimney Cycle Analysis with System Loss and Solar collector Performance. ASME Journal of Solar Energy Engineering, Vol. 122, pp. 133-137, August 2000.

Granger R. A., (1988), Experiments in fluid mechanics, Holt, Rinehart and Winston, Inc., USA

Grotjans H. and Menter F.R., Wall functions for general application CFD codes, ECCOMAS 98 Proceedings of the fourth European computational fluid dynamics conference, John Wiley \& Sons, pp. 1112-1117, 1998 
Haaf, Friedrich, Mayr and Schlaich, Solar Chimneys - Part I: Principle and Construction of the Pilot Plant in Manzanares, International Journal Solar Energy, 1983, Vol.2, pp. 3-20

Haaf, W, SCPPS - Part II: Preliminary Test Results from the Manzanares Pilot Plant, International Journal Solar Energy, 1984, Vol. 2, pp. 141-161

Habashi W. G., Dompierre J., Bourgault Y., Fortin M. and Vallet M.-G., Certifiable computational fluid dynamics through mesh optimization, AAIA journal, Vol. 36, No. 5, May 1998, pp. 703-711

Hall. P, Taylor-Gortler vortices in fully developed or boundary-layer flows: linear theory, Journal of Fluid Mechanics, 1982, Vol. 124, pp. 475-494

Houghton and Carpenter (1993), Aerodynamics for Engineering Students, 4th Edition, Butterworth Heinemann,

Japikse, D. Advanced experimental techniques in turbomachinery, Principal lecture series, 1986

Jog M. G., Physical dimention ratios of Kaplan turbines, Water power and dam construction, Vol. 28, No. 3, pp. 36-38

Kirstein C.F. (2001), Design of inlet guide vanes and modelling of a solar chimney inlet, Final year thesis, Department Mechanical Engineering, University of Stellenbosch

Kröger D.G. and Buys J.D., Solar chimney plant performance characteristics, South African Institution of Mechanical Engineering, R and D Journal, 2 July 2002, Vol.18, pp. 31-36

Lacovides H. and Theofanopoulos I. P., Turbulence modelling of axisymmetric flow inside rotating cavities, International Journal of Heat and Fluid Flow, Vol.12, No.1, March 1991, pp. 2-11

Launder B.E. and Spalding D.B., The numerical computation of turbulent flows, Comp Meth Appl Eng 3:269-289, 1974

Lebas G. (2001), Solar Chimney, Vacation work report, University of Stellenbosch, South Africa

Lugaresi A. and Massa A., Kaplan turbines: design trends in the last decade, Water power and dam construction, May 1988, pp.12-17

Magauer P. F. and Wolfartsberger K., Design concepts for vertical Kaplan turbines, Waterpower ' 91 : Proceedings of the international conference on hydropower, 24-26 July 1991, Vol.3, pp. 2109-2118. 
Menter F. R., Two-equation eddy-viscosity turbulence models for engineering applications, AIAA journal, Vol. 32, No.8, 1994, pp. 1598-1605

Millandri G (2003). Solar chimney multiple turbine performance, Final year thesis, Department Mechanical Engineering, University of Stellenbosch

Oberkampf W. L. and Blottner F. G., Issues in computational fluid dynamics code verification and validation, AIAA Journal, Vol. 36, No. 5, May 1998, pp. 687-695.

Pasumarthi and Sherif, Experimental and Theoretical Performance of a Demonstration SCPP Model - Part I: Mathematical Model Development, International Journal of Energy Research, 1998, 22, pp. $277-288$

Pasumarthi and Sherif, Experimentation and Theoretical Performance of a Demonstration SCPP Model - Part II: Experimental and Theoretical Results and Economic Analyses, International Journal of Energy Research, 22, pp. 443-461 (1998)

Pillay L.A., (2002), Investigation of tall chimney internal drag loss, Final year thesis, Department Mechanical Engineering, University of Stellenbosch

Pylev I. M., Abdurakhmanov L. F., Sotnikov A. A., New technical guidelines for large vertical-shaft Kaplan and mixed flow hydroturbines, Soviet energy technology, No.5, 1986, pp. 5-11

Rao M. S. and Hassan H. A., Modeling turbulence in the presence of adverse pressure gradients, Journal of aircraft, Vol.35, No.3, 1998, pp. 500-502

Rizzi A. and Vos J., Toward establishing credibility in computational fluid dynamics simulations, AAIA journal, Vol. 36, No. 5, May 1998, pp. 668-675

Roache P. J., Verification of codes and calculations, AAIA journal, Vol. 36, No. 5, May 1998, pp. 696-702

Roth A. D., Case studies in upgrading Francis and Kaplan turbines for improved hydraulic performance, ASME, 94-JPGC-PWR-5, October 1994

Rumsey C. L. and Vasta V. N., Comparison of the predictive capabilities of several turbulence models, Journal of aircraft, Vol.32, No.3, May-June 1995, pp. 510-514

Schlaich, Jörg, Tension structures for solar energy generation, Engineering structures, 21, 1999, pp. 658-668

Schlaich, Jörg, The Solar Chimney - Electricity from the sun, 1995 Edition Axel Menges, Stuttgart

Sayers, A.T. (1990), Hydraulic and Compressible Flow Turbomachines, McGrawHill, London 
Schilling R., Knapp W., Bader R., Fritz J. And Mochkaai Y., Experimental and theoretical loss analysis in a Kaplan turbine, International journal on hydropower and dams, Vol. 5 nr. 6, 1998, pp. 63-67

Schweiger F. and Gregori J., Developments in the design of Kaplan turbines, Water power and dam construction, Nov 1987, pp.16-20

Schweiger F. and Gregori J., Design data of Kaplan and bulb turbine units, Waterpower '89: Proceedings of the international conference on hydropower, 23-25 August 1989, pp. 1387-1396

Stratford, B.S., (1959), The Prediction of Separation of the Turbulent Boundary Layer, Journal of Fluid Mechanics, Vol. 5, part I.

Strohmaier C. K-H., (1997), An evaluation of a scale model medium speed windtunnel, Masters thesis, Department mechanical engineering, University Stellenbosch

Suga K., Nagaoka M., Horinouchi N., Abe K. and Kondo Y., Application of a threeequation cubic eddy viscosity model to 3-D turbulent flows by the unstructured grid method, International journal of heat and fluid flow, Vol.22, 2001, pp. 259-271

Thomas K. (2003), Personal correspondence, University of Stellenbosch, South Africa.

Trieb F., Langniß O. and Klaiß H., Solar Electricity Generation - A Comparative View of Technologies, Costs and Environmental Impact, Solar Energy 1997, Vol. 59, pp. 89-99

Uys J., Integrasie toepassings, Haum-Tersiêr, 1990

Von Backström, T.W. (2002-2004) Personal correspondence, University of Stellenbosch, South Africa.

Von Backström T.W. and Gannon A. J., Solar Chimney Turbine

Characteristics. ISES Solar Energy Journal, Special proceedings Issue, Volume 76, Issues 1-3, pp. 235-241, January - March 2004.

Von Backström T.W., Gannon A. J. and Bernhardt A., Pressure Drop in solar power plant chimneys. ASME Journal of Solar Energy Engineering, Vol. 125, No. 2, pp. 165-169, May 2003.

Von Backström, T.W., Kirstein, C.F. and Pillay, L.A., The influence of some secondary effects on solar chimney power plant performance, International Solar Energy Society, 2003 Solar World Congress, ISES 2003, Göteborg, Sweden, June 1619, 2003.

Von Backström T.W. and Gannon A. J., Solar Chimney Air Standard Thermodynamic Cycle, SAIMechE R\&D Journal, Vol 16, No. 1 pp. 16-24, 2000 
Walpole, Meyers, Meyers (1998), Probability and Statistics, $6^{\text {th }}$ addition, Prentice Hall international edition.

White, F.M. (1999), Fluid Mechanics, 4th Edition, McGraw-Hill, Singapore

White F. M., (1991), Viscous fluid flow, $2^{\text {nd }}$ edition, McGraw-Hill International editions, New York

Zarea S., Theodorescu C., New criteria for the standardization of Kaplan turbines, ASME FEDSM'97, Vol.3, June 22-26, 1997, 


\section{APPENDIX A: CAlCulations}

\section{A.1 Boundary Layer Analysis of the OUter Wall}

CFX is not capable of analysing transitional flow: the flow is either solved as being fully laminar or fully turbulent. It is therefore necessary to do a boundary layer analysis on the flow behaviour to validate the CFD predictions.

To do the initial analysis the flow is assumed to be laminar from the entry at the deck perimeter onwards. Then the development of the necessary parameters required to predict flow separation and turbulence transition is calculated. To calculate the point of separation four methods where evaluated. For the transition to turbulence two methods were applied and compared.

A 2D finite difference laminar boundary method could be implemented. This method is very accurate, but applying it to this problem is arduous. The method requires known conditions outside the boundary layer, which can only be obtained from detailed CFD analyses. If such detailed analyses are done, then the 2D solution method becomes obsolete. If these conditions are estimated, then the magnitude of the error is unknown. The only other option is to build a grid of the entire flow regime, which is beyond the scope of this project. Therefore it was decided to use a simpler $1 \mathrm{D}$ method such as an integral method.

Aungier (2000) suggests a simple method calculated from the momentum integral equation, but this requires the user to know a boundary value of the momentum thickness, and the problem is solved implicitly. For even simple flow regimes, this method converges slowly and therefore a better method to analyse the flow is required.

The third option is suggested by White (1991): the correlation method of Thwaites. The correlation method of Thwaites is accurate to within $5 \%$ of the exact solution for mild adverse gradients and 15\% near the separation point (White, 1991). The fourth option is the Stratford (1959) method. The method of Thwaites is evaluated along with the Stratford method to the exact solution in table 4-5 of White (1991) for eleven 
classical separating-flow free stream velocity distributions. Generally the Stratford (1959) method is more accurate, but the Thwaites method is always within 5\% of the answer and requires less knowledge of the flow properties. Both methods solve the answers explicitly, which makes them faster than the slowly converging method of Aungier (2000).

Considering all the advantages and disadvantages of the methods and the convenience for following calculations, the method of Thwaites is chosen to be the most appropriate for this problem. Thwaites is easy to implement, robust, accurate enough and it calculates properties that are useful for further evaluation.

The outer wall pressures that were obtained in CFX correlated accurately enough with the experimental results to be used with confidence (see for instance Figure B-52). To obtain a CFD solution on a fine mesh is expensive and hence large discontinuities in the pressure gradient could exist. To correct this situation, the data was smoothed using a piece-wise cubic spline curve and interpolation.

The static pressure is assumed to be constant over the boundary layer; therefore the wall pressure can be converted to the free stream velocity using Bernoulli

$$
U=\sqrt{\frac{2 \cdot p}{\rho}}
$$

Once the velocity distribution and flow properties are known, the momentum thickness can be calculated explicitly to within 3\% accuracy using the simple quadrature for axisymmetric flow.

$$
\theta^{2} \approx \frac{0.45 v}{r^{2} U^{6}} \int_{0}^{x} r^{2} U^{5} d x
$$

where $\mathbf{r}$ is radial co-ordinate, $\mathbf{U}$ the free stream velocity and $\mathbf{x}$ the developed length. To obtain this from numeric data, equation ( A-2) can be discretised using a backward difference method as follows: 


$$
\theta_{i}^{2} \approx \frac{0.45 v}{r_{i}^{2} U_{i}^{6}} \sum_{j=2}^{i} r_{j}^{2} U_{j}^{5}\left(x_{j}-x_{j-1}\right)
$$

next the velocity gradient is calculated by a backward difference method as follows:

$$
U^{\prime}=\frac{d U_{i}}{d x}=\frac{U_{i}-U_{i-1}}{x_{i}-x_{i-1}}
$$

Thwaites defined the parameter $\lambda$ as

$$
\lambda=\frac{\theta^{2} \cdot U^{\prime}}{v}
$$

so that the shear correlation, $S(\lambda)$, and the shape-factor correlation, $H(\lambda)$, can be determined by (White, 1991)

$$
\begin{aligned}
& S(\lambda)=(\lambda+0.09)^{0.62} \\
& H(\lambda) \approx 2.0+4.14 z-83.5 z^{2}+854 z^{3}-3337 z^{4}+4576 z^{5}
\end{aligned}
$$

where $z=0.25-\lambda$. The momentum integral equation for axisymmetric flow is written as

$$
\frac{C_{f}}{2}=\frac{1}{U^{2}} \frac{\partial}{\partial t}\left(U \delta^{*}\right)+\frac{2 \theta+\delta^{*}}{U} \frac{\partial u}{\partial x}+\frac{\partial \theta}{\partial x}+\frac{\theta}{r} \frac{d r}{d x}-\frac{v_{w}}{U}
$$

Only steady 2D flow with no wall suction is considered, therefore equation ( A-8) may be simplified to:

$$
\frac{C_{f}}{2}=\frac{2 \theta+\delta^{*}}{U} \frac{d u}{d x}+\frac{d \theta}{d x}+\frac{\theta}{r} \frac{d r}{d x}
$$

Using the relation $\mathrm{H}=\delta^{*} / \theta$, the friction factor may be written as:

$$
C_{f}=2\left[\frac{d \theta}{d x}+(2+H) \frac{\theta \cdot U^{\prime}}{U}+\frac{\theta}{r} \frac{d r}{d x}\right]
$$


which can be discretised as

$$
C_{f, i}=2\left[\frac{\theta_{i}-\theta_{i-1}}{x_{i}-x_{i-1}}+\left(2+H_{i}\right) \frac{\theta_{i} \cdot U_{i}{ }^{\prime}}{U_{i}}+\frac{\theta_{i}}{r_{i}} \frac{\left(r_{i}-r_{i-1}\right)}{\left(x_{i}-x_{i-1}\right)}\right]
$$

Flow separation is predicted where $\mathrm{H}=3.55, \lambda \approx-0.09$ or when $\mathrm{C}_{\mathrm{f}}=0$. The methods were programmed using Matlab ${ }^{\mathrm{TM}} \mathrm{R} 12$ and the results are shown in Figure A-1 to Figure A-3 and in Table A-1 on page A-8. The developed distance starts at the perimeter of the deck and grows as a particle migrates along the outer wall.

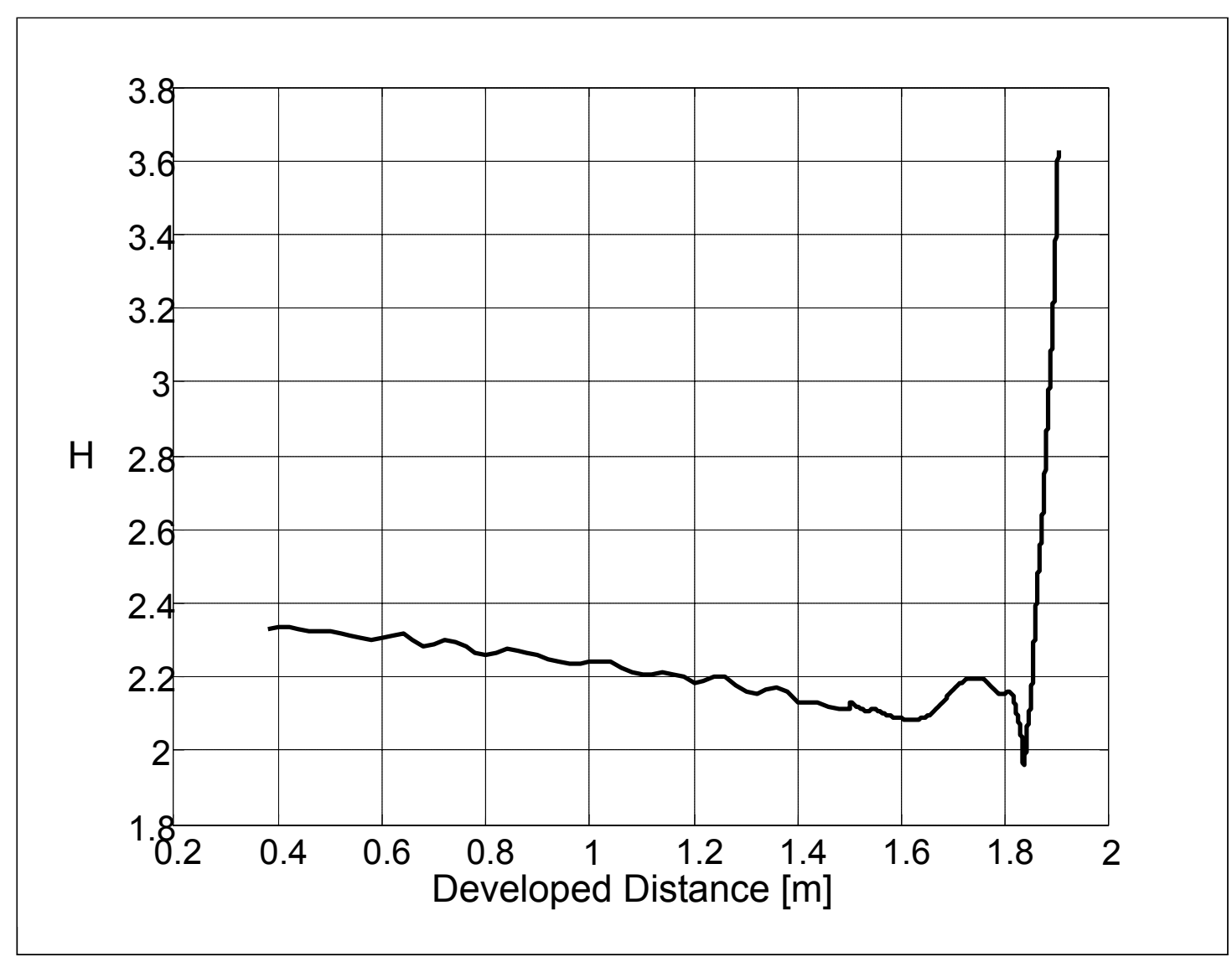

Figure A-1 - Form factor development 


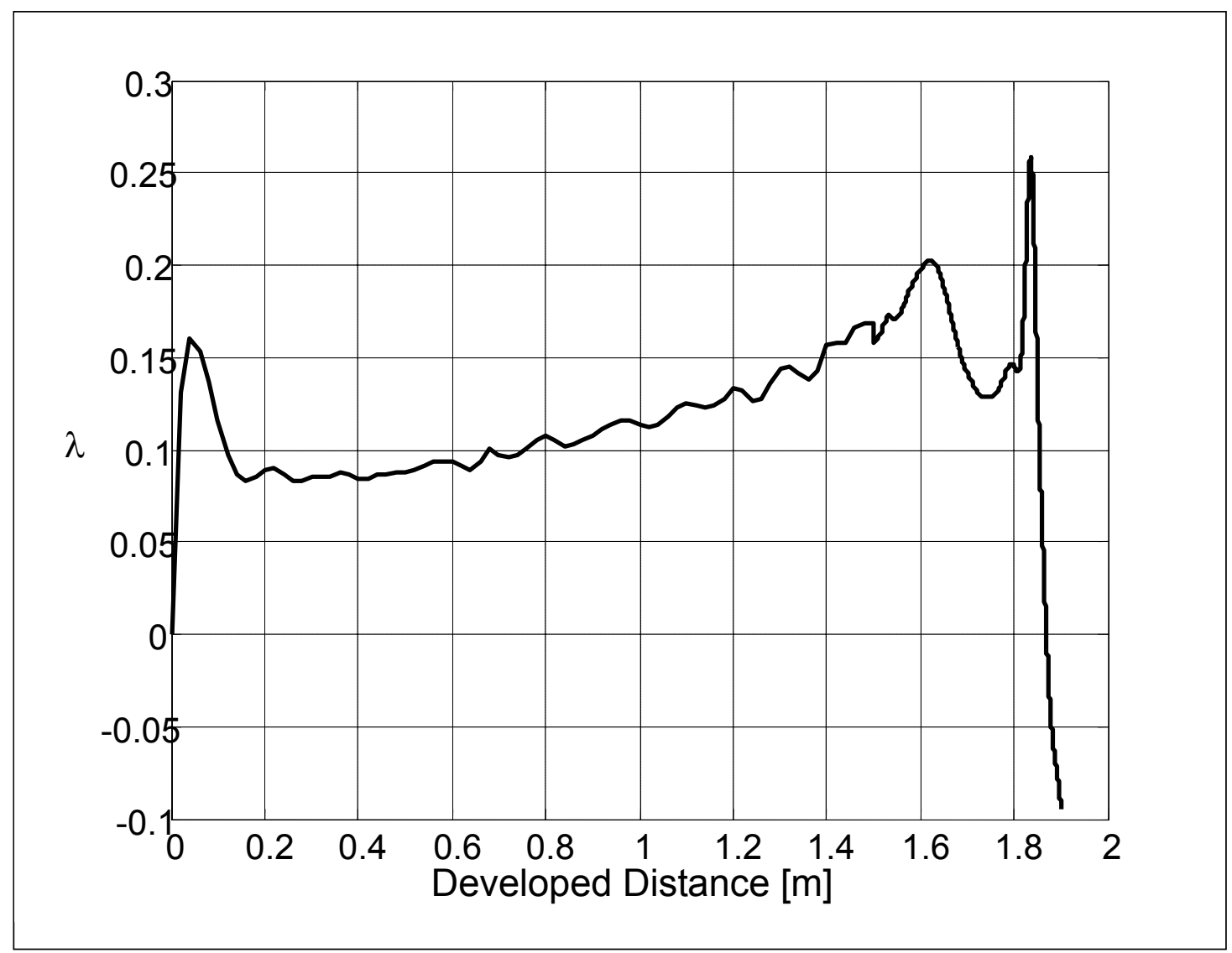

Figure A-2 - Thwaites parameter's development

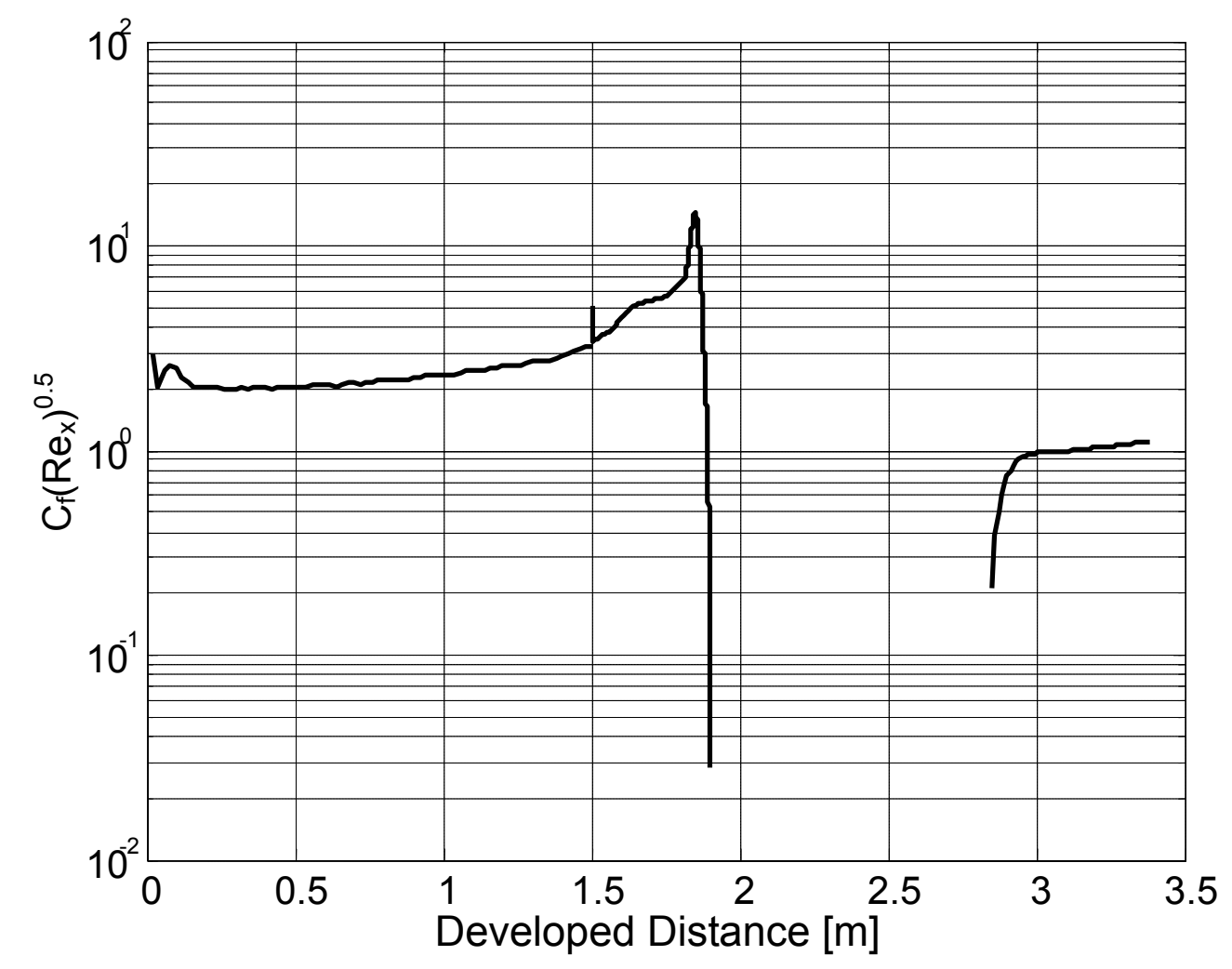

Figure A-3 - Friction coefficient development

A- 5 
The next step is to predict the transition to turbulence. As mentioned, CFX does not predict transition to turbulence. If such knowledge could be obtained, the flow behaviour prediction could be modelled even better. Aungier (2000) suggests that the flow is turbulent when $\operatorname{Re}_{\theta}>250$. This method is too crude for this analysis; hence two popular methods are used and compared, namely the one step method of Michel and the one step method of Wazzan et al. (White 1991).

The first method is the one step method of Michel. The method is simple by having to ignore the point of instability and simply compute the momentum thickness. Here Thwaites solution is useful where as Stratford would have required additional calculations. Michel's transition line is defined as:

$$
\operatorname{Re}_{\theta, t r} \approx 2.9 \operatorname{Re}_{x, t r}^{0.4}
$$

The $\operatorname{Re}_{\theta}(\mathrm{x})$ approaches the transition line from below and intersects it at the transition point. The result is shown in Figure A-4 and Table A-1.

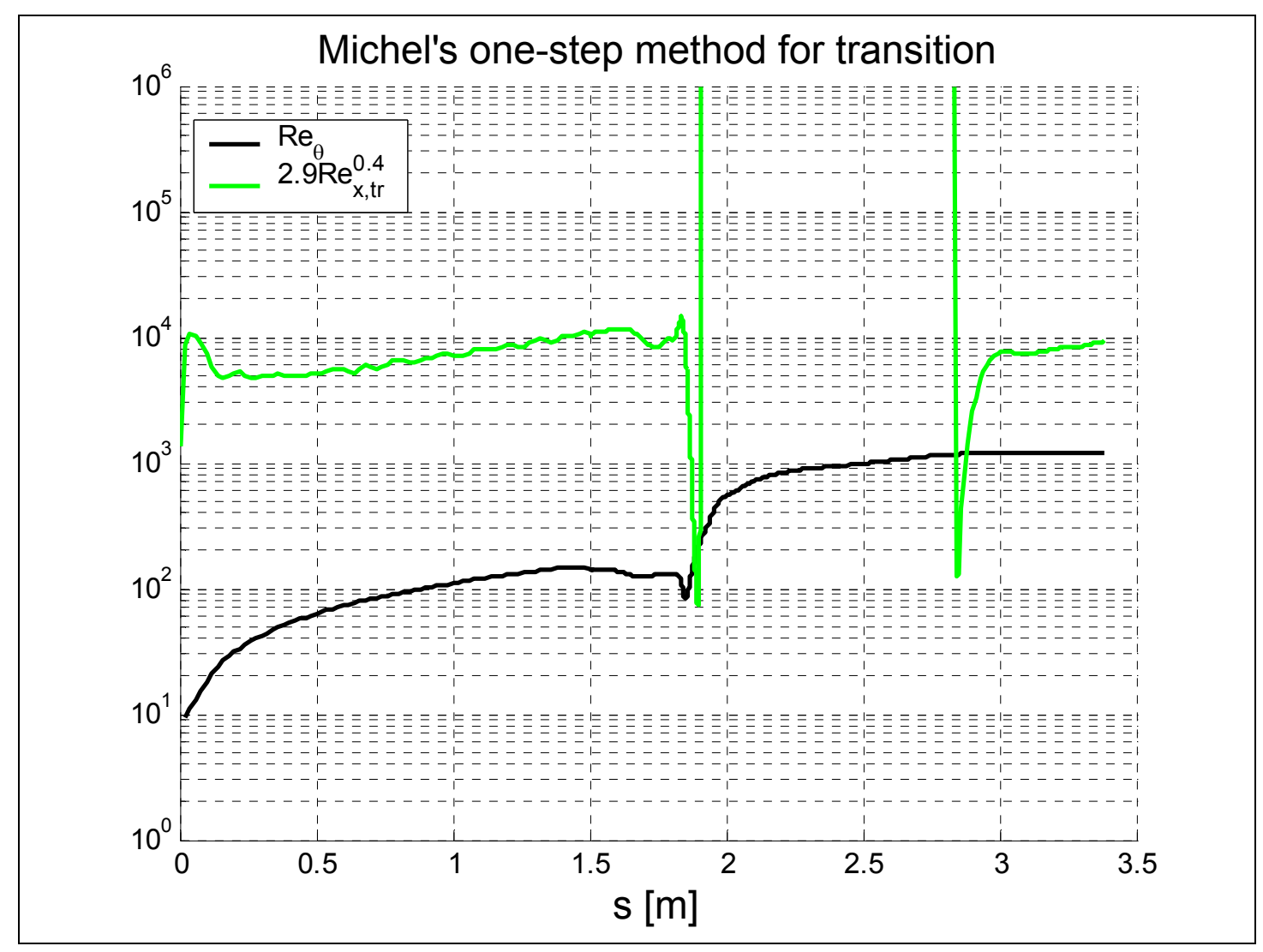

Figure A-4 - Michel's method for transition prediction 
The second method is the one step method of Wazzan et al.. To determine the transition point $H(x)$ is calculated and where the locus of $H\left(\operatorname{Re}_{x}\right)$ for $H \varepsilon[2.1,2.8]$ intersects Wazzan's suggested curve for transition defined as:

$$
\log _{10}\left(\operatorname{Re}_{x, t r}\right) \approx-40.4557+64.8066 H-26.7538 H^{2}+3.3819 H^{3}
$$

Wazzan's result is shown in Figure A-5. The developed distance value can be determined from the form factor in Figure A-1.

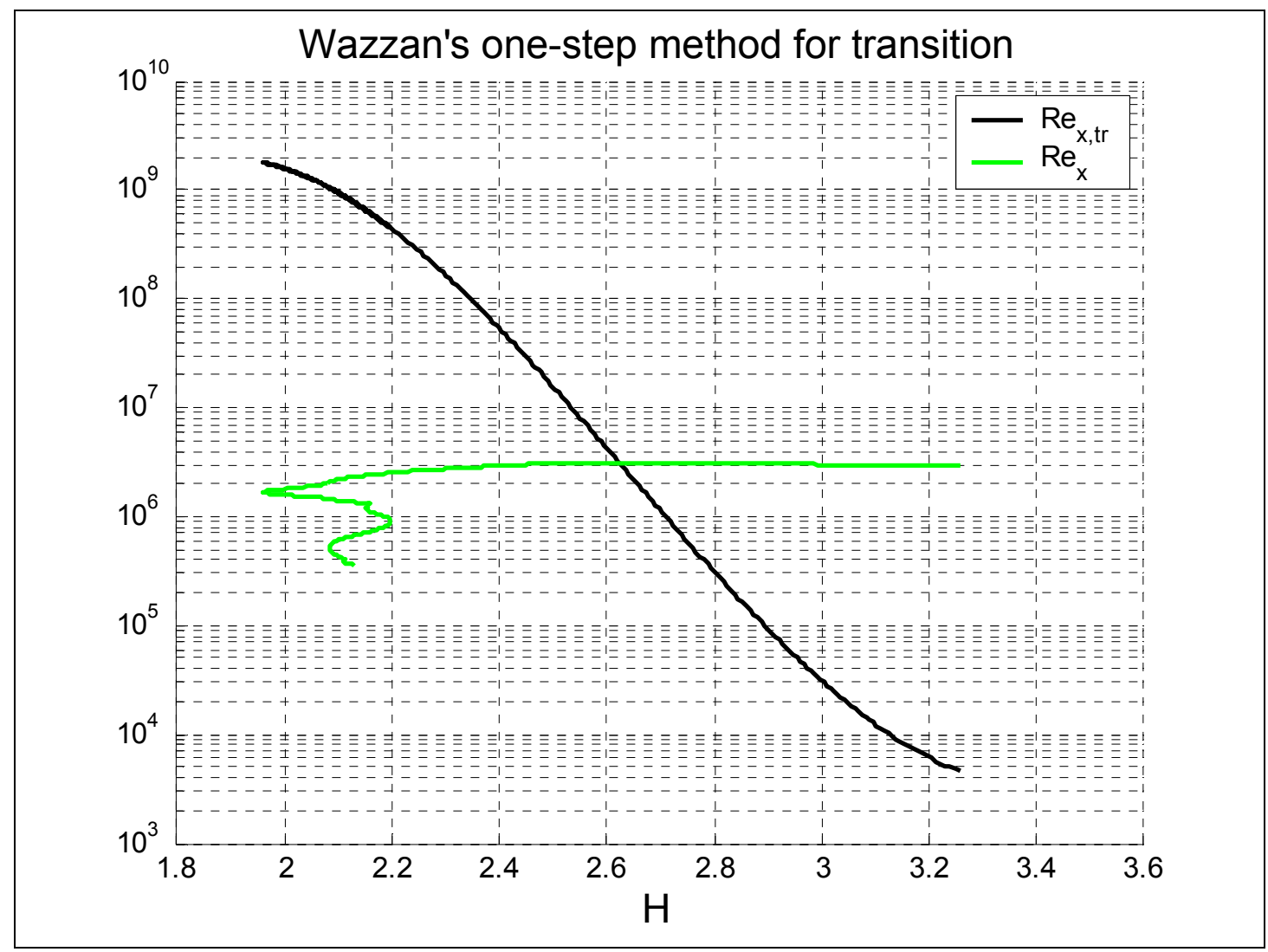

Figure A-5 - Wazzan's method for transition prediction

In White (1991) the methods for determining the transition points were compared for flows ranging from adverse gradients to very strong favourable gradients. All methods compare well for the adverse gradients, but differ by orders of magnitude for favourable pressure gradients. White (1991) recommends that the more modern method of Wazzan is used in cases of strong favourable pressure gradients. Therefore the bias would lean towards the answer obtained by Wazzan, although it would be helpful to have another method verify whether the results are in the right region. 
The separation- and the transition points, shown in Table A-1, are $3 \mathrm{~cm}$ from each other, with turbulence occurring first. The distance between separation and transition points are well within the limit of accuracy of these methods; therefore it was decided to verify the results by observing what the CFD predicts for laminar flow.

Table A-1 - Separation and transition locations from edge of upper deck

\begin{tabular}{|c|c|c|}
\cline { 2 - 3 } \multicolumn{1}{c|}{} & Method used & Distance [m] \\
\hline \multirow{4}{*}{ For Separation } & $\mathrm{H}=3.55$ & 1.8995 \\
\cline { 2 - 3 } & $\lambda=-0.09$ & 1.8989 \\
\cline { 2 - 3 } & $\mathrm{C}_{\mathrm{f}}=0$ & 1.8985 \\
\cline { 2 - 3 } & $\mathrm{CFX}$ & 1.8965 \\
\hline \multirow{2}{*}{ For Transition } & $\mathrm{Re}_{\theta}=250$ & 1.90979 \\
\cline { 2 - 3 } to turbulence & Michel & 1.88487 \\
\cline { 2 - 3 } & Wazzan & 1.87036 \\
\hline
\end{tabular}

CFD was done for fully laminar flow on a similar grid to the one used to obtain the results for Thwaites. The CFD method predicts flow separation for laminar flow at a developed distance of $1.8965 \mathrm{~m}$. This is $2 \mathrm{~mm}$ short of the Thwaites results, which is an excellent agreement, and hence the results of Thwaites are used confidently.

There is the slight possibility that the flow may experience laminar separation before it becomes turbulent and reattaches to the wall, but thus far all the analyses and observations suggest otherwise.

The CFD turbulence results were further verified using the compressor code developed by Thomas (2003). The results of the turbulent flow analyses in CFD and with this compressor code are not shown here, but both predict that the flow will not separate from the wall. 


\section{A.2 InVESTIGATION OF THE IGV BLADE ROW TORQUE}

The torque on the IGV-blade-row can be determined accurately by integrating the forces of the static pressures on the blade surfaces. These results can be obtained from CFD analyses. Unfortunately obtaining the answer in such a manner is expensive; therefore it is beneficial to have an inexpensive analytical model that can predict the causes of any changes for the purpose of optimization.

The torque can be simply determined by observing the change in angular momentum of the fluid as it passes through the blade row, by applying the Euler equation. Firstly the assumption is made that, even for the full scale plant, the flow could be considered incompressible through the IGVs.

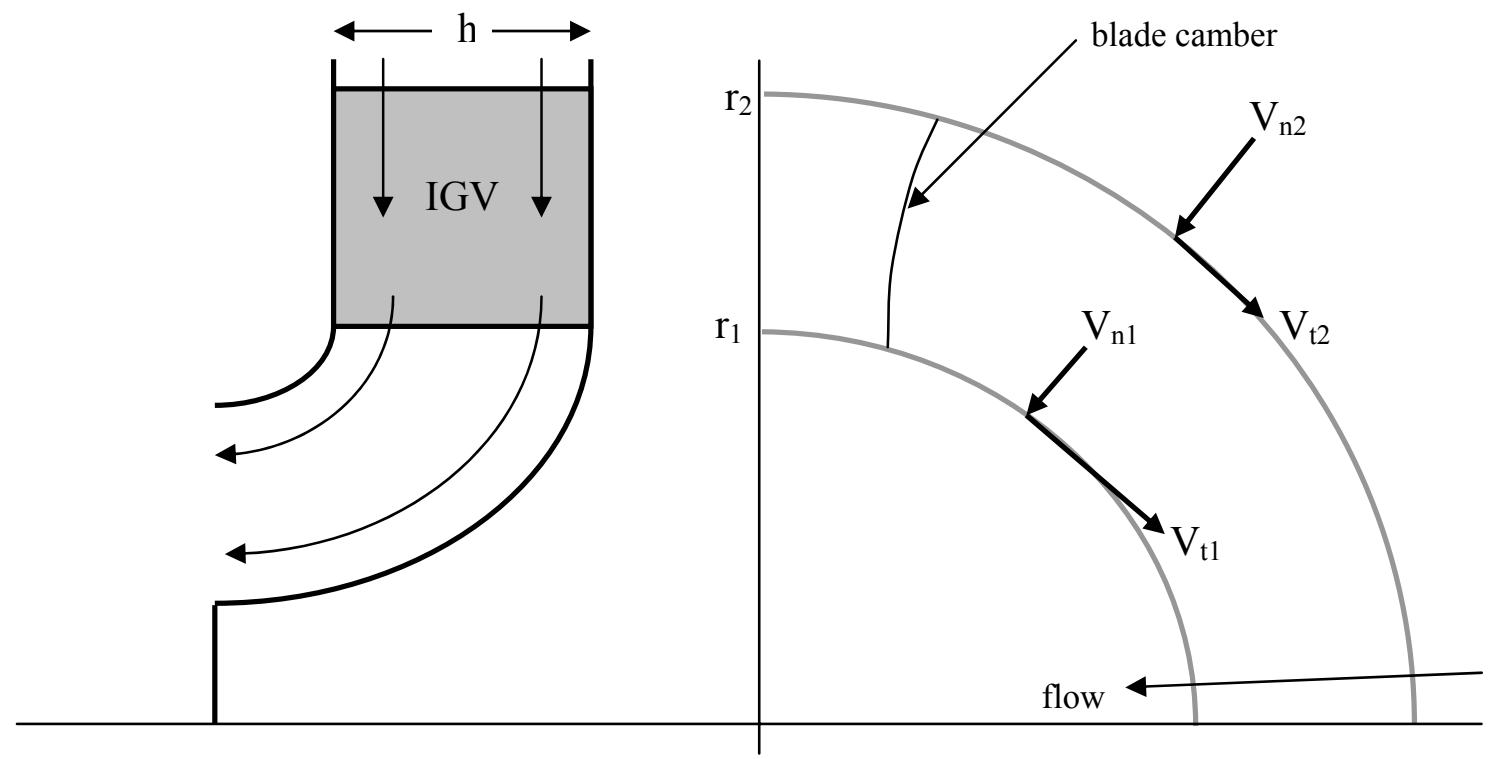

Figure A-6 - IGV cascade schematic

From the law of conservation of angular momentum the torque can be written as the sum of the moments at the entry and exit of the blade row, as follows.

$$
\sum \vec{M}=\vec{T}=\left(\vec{r}_{2} \times \vec{V}_{2}\right) \cdot \dot{m}-\left(\vec{r}_{1} \times \vec{V}_{1}\right) \cdot \dot{m}
$$

where

$$
\dot{m}=\rho \cdot Q
$$

Now

$$
\vec{r}_{2} \times \vec{V}_{2}=r_{2} \cdot V_{t 2} \cdot \sin 90^{\circ} \cdot \vec{k}=r_{2} \cdot V_{t 2} \cdot \vec{k}
$$


and

$$
\vec{r}_{1} \times \vec{V}_{1}=r_{1} \cdot V_{t 1} \cdot \vec{k}
$$

therefore

$$
\vec{T}=\rho \cdot Q \cdot\left(r_{2} \cdot V_{t 2}-r_{1} \cdot V_{t 1}\right) \cdot \vec{k} \quad \text { clockwise }
$$

It can be safely assumed that the flow enters the blade row radially at $r_{2}$, therefore the tangential component $\mathrm{V}_{\mathrm{t} 2}=0$. The flow that exits the blade row at $\mathbf{r}_{1}$ has an exit angle of $\varphi$, therefore

$$
V_{t 1}=|\vec{V}| \cdot \sin \phi
$$

but

$$
|\vec{V}|=\frac{Q}{A}
$$

and

$$
A=2 \cdot \pi \cdot r_{1} \cdot h
$$

therefore

$$
\vec{T}=-\rho \cdot Q \cdot r_{1} \cdot \frac{Q}{2 \cdot \pi \cdot h \cdot r_{1}} \cdot \sin \phi \cdot \vec{k} \quad \text { clockwise }
$$

or simply

$$
\vec{T}=\frac{\rho \cdot Q^{2}}{2 \cdot \pi \cdot h} \cdot \sin \phi \approx \frac{\rho \cdot Q^{2}}{2 \cdot \pi \cdot h} \cdot \sin \beta
$$

Note that this assumes that the blade angle and flow exit angle are equal, although it is not entirely true. The flow exit angle was compared to the IGV angle, adjusted with the tail flap, in Kirstein (2001). This yielded the following results:

Table A-2 - IGV set angle versus flow deflection

\begin{tabular}{|c|c|c|}
\hline $\boldsymbol{\beta}$ & $\boldsymbol{\varphi}$ & $\boldsymbol{\Delta}\left[{ }^{\circ}\right]$ \\
\hline 37.50 & 37.43 & 0.07 \\
\hline 32.50 & 32.52 & 0.02 \\
\hline 22.50 & 22.49 & 0.01 \\
\hline 12.50 & 12.32 & 0.18 \\
\hline 7.50 & 7.23 & 0.27 \\
\hline 2.50 & 2.16 & 0.34 \\
\hline
\end{tabular}

The table above shows that the assumption $\beta \approx \varphi$ is accurate enough for any purpose in this project. 


\section{A.3 LOAD-TO-STIFFNESS RATIO OF THE IGV CASCADE}

To determine the improvement in torsional strength in the SCPP, a simple analysis is done on a single IGV.

Assume that the load of the chimney is evenly distributed over the whole of the IGV and that torsion from the turning flow acts as a moment in the tangential direction for an IGV so that the analysis can be done as follows:

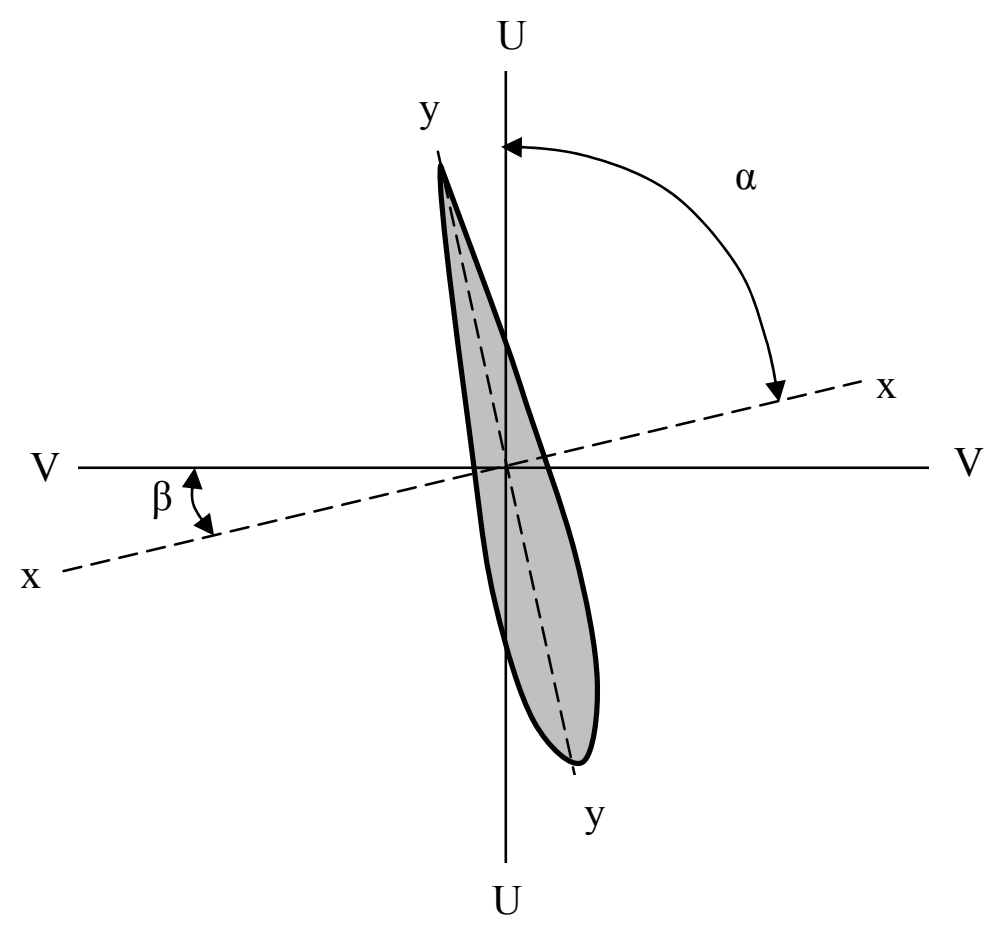

Figure A-7 - IGV stagger orientation schematic

The UU-axis is perpendicular to the tangential. For this analysis it is assumed that a moment acts across this axis. If the material and profile shape is kept as constants, then the stiffness of the cascade can be investigated by observing the variation of $\mathrm{I}_{\mathrm{uu}}$. Once the stiffness of the structure is determined, it can be compared with the load carried by the blade row.

Uys et al. (1990) defines the area moment over a skew axis in 3D. By rewriting it for a $2 \mathrm{D}$ case the equation becomes: 


$$
I_{u u}=\lambda^{2} I_{x x}+\mu^{2} I_{y y}+\lambda \mu I_{x y}
$$

where $\mu=\cos (\beta)$ and $\lambda=\cos (\alpha)$ for this case. The profile thickness of a 4 digit NACA profile is defined in Houghton and Carpenter (1993) as

$$
y_{t}= \pm 5 c t\left[0.2969 \sqrt{\xi}-0.1260 \xi-0.3516 \xi^{2}+0.2843 \xi^{3}-0.1015 \xi^{4}\right]
$$

where $\mathbf{t}$ is the maximum thickness and $\xi$ is the ratio of the horizontal position relative to the chord length $\mathbf{x} / \mathbf{c}$. The leading edge radius is defined as

$$
r_{t}=1.1019 c t^{2}
$$

If $\mathbf{p}$ is the position of the maximum camber, $\mathbf{m}$ is the maximum camber, then the camber line is defined as

$$
\begin{array}{ll}
y_{c}=\frac{m c}{p^{2}}\left(2 p \xi-\xi^{2}\right) & : \xi \leq \mathrm{p} \\
y_{c}=\frac{m c}{(1-p)^{2}}\left[(1-p)+2 p \xi-\xi^{2}\right] & : \xi \geq \mathrm{p}
\end{array}
$$

The blade profile geometry of the NACA 4-digit profile for the IGVs is given by Gannon (2002) and is repeated here for convenience.

\section{Table A-3 - IGV NACA parameters}

\begin{tabular}{|c|c|}
\hline & Of the chord \\
\hline $\mathbf{m}$ & $2.64 \%$ \\
\hline $\mathbf{p}$ & $29.85 \%$ \\
\hline $\mathbf{t}$ & $11.7 \%$ \\
\hline $\mathbf{m}_{\mathbf{t}}$ & $28.58 \%$ \\
\hline $\mathbf{r}_{\mathbf{t}}$ & $5.47(6$ is standard) \\
\hline
\end{tabular}




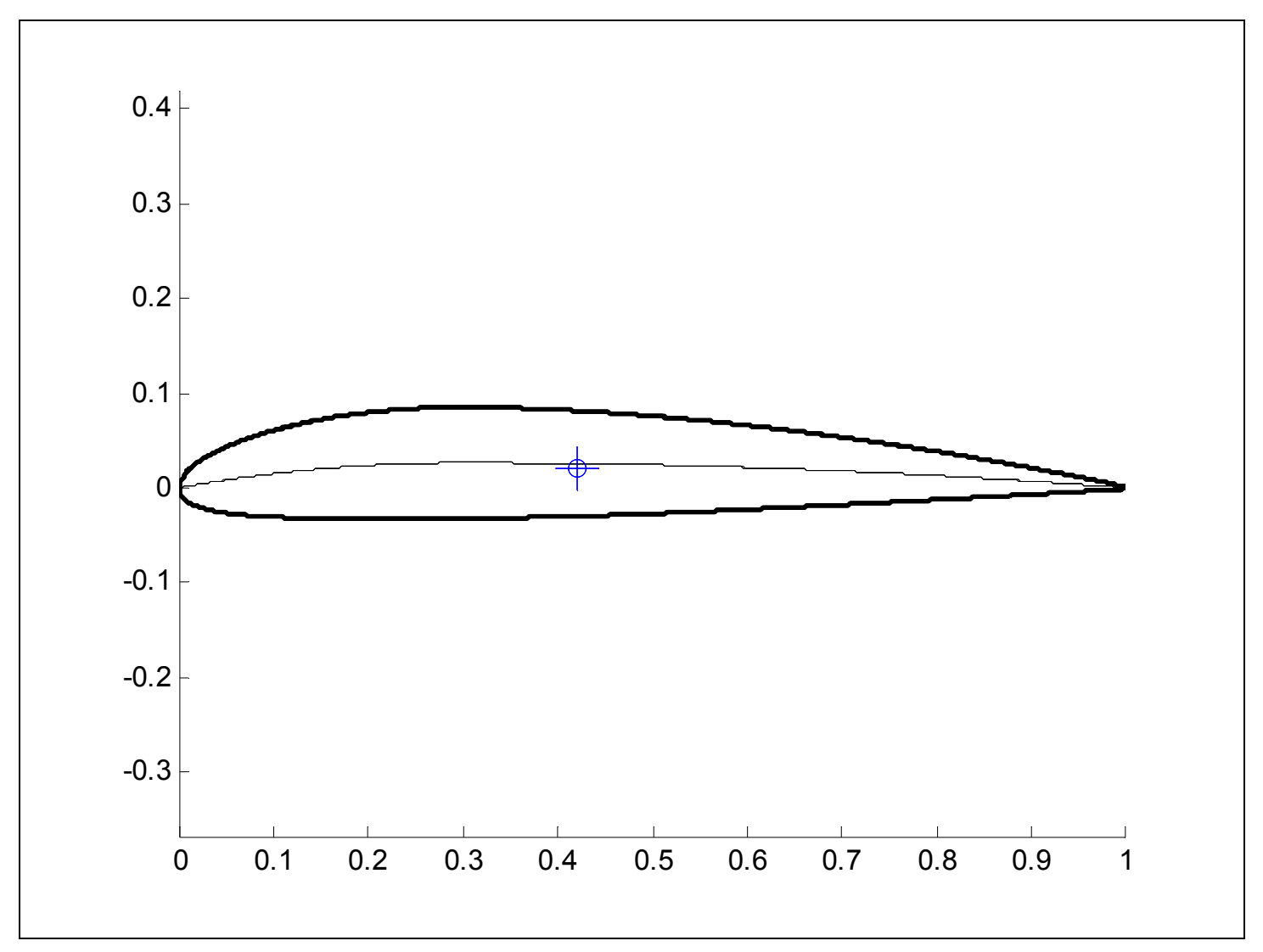

Figure A-8 - IGV profile with centre of gravity

The section properties were determined by a program written in Matlab R12 ${ }^{\mathrm{TM}}$. The profile was broken up into a series of rectangles and triangles and integrated by summing the influence of each of these sections.

Firstly the area is determined by using the trapezium method. From the area the centre of gravity for the profile is determined.

$$
\bar{x}=\frac{\int_{A} x d A}{A} \quad \text { and } \quad \bar{y}=\frac{\int_{A} y d A}{A}
$$

The area moments are defined as

$$
I_{x x}=\int_{A} y^{2} d A \quad I_{y y}=\int_{A} x^{2} d A \quad I_{x y}=\int_{A} x y d A
$$

To sum the influence of each increment, the following equations are used 


$$
\begin{aligned}
& I_{x x}=\sum\left(I_{x x}^{\prime \prime}+A \cdot\left(y_{c}^{\prime \prime}-\bar{y}\right)^{2}\right) \\
& I_{y y}=\sum\left(I_{y y}^{\prime \prime}+A \cdot\left(x_{c}^{\prime \prime}-\bar{x}\right)^{2}\right) \\
& I_{x y}=\sum\left(I_{x y}^{\prime \prime}+A \cdot\left(y_{c}^{\prime \prime}-\bar{y}\right) \cdot\left(x_{c}^{\prime \prime}-\bar{x}\right)\right)
\end{aligned}
$$

where the " denotes the incremental area and the subscript $\mathbf{c}$ the centre of gravity for that incremental area.

For the program a finite number of panels are given to calculate the section properties and hence the property values could become expensive to calculate accurately, since the properties converge very slowly to an answer (approximately 10000 panels give an answer that has less than $1 \%$ error on all the properties).

The method for extrapolating a variable $\varphi$ to zero grid size, using the results on three grids that are successively refined by a factor of 2 , is done as follows.

$$
\varphi \approx \varphi_{h}+\alpha \cdot h^{n}
$$

where

$$
n=\log _{2}\left(\frac{\varphi_{2 h}-\varphi_{4 h}}{\varphi_{h}-\varphi_{2 h}}\right)
$$

and

$$
\alpha \cdot h^{n}=\frac{\left(\varphi_{h}-\varphi_{2 h}\right)}{2^{n}-1}
$$

Substituting equation ( A-33) into ( A-34) yields

$$
\alpha \cdot h^{n}=\frac{\left(\varphi_{h}-\varphi_{2 h}\right)^{2}}{2 \varphi_{2 h}-\varphi_{h}-\varphi_{4 h}}
$$

Substitute equation ( A-35) back into equation ( A-32) to obtain

$$
\varphi \approx \frac{\varphi_{2 h}^{2}-\varphi_{h} \cdot \varphi_{4 h}}{2 \varphi_{2 h}-\varphi_{h}-\varphi_{4 h}}
$$


Note that equation ( A-36) is only valid if the variable $\boldsymbol{\varphi}$ converges as the grid is refined. Fortunately that condition is inherent of the nature of this problem. The program was then adapted so that the user could choose a relatively small amount of panels that will serve as the coarse mesh and then it calculates the zero grid size results.

From equation ( A-23) it was calculated that if the blade angle is the only variable, then the load on the blades varies sinusoidally with the flow turning angle. Substituting the profile properties into equation ( A-24), the stiffness of the cascade is determined for each blade angle setting.

The load-to-stiffness ratio is defined as

$$
\mathrm{L}-\mathrm{S}=\Lambda \frac{\text { Load }}{\text { Stiffness }} \propto \frac{\text { Load }}{\text { Stiffness }}
$$

$\boldsymbol{\Lambda}$ is a symbol denoting a constant value depending on the scale and shape of the blades, the deck height, flow rate etc. of the plant. Since only the effect of changing the blade angles are investigated at the moment, this value can be thoughtfully ignored to normalise the load-to-stiffness ratio.

The load is proportional to the load on the blades due to change in angular momentum and the stiffness is proportional to $\mathrm{I}_{\mathrm{uu}}$. It can be seen that a lower value for the load-tostiffness ratio is more desirable for the structure. The results are shown in Figure A-9.

These results demonstrate that the structure's stiffness is compromised most heavily at a turning angle of $10^{\circ}$. However for this thesis only two angles are considered, the $22.5^{\circ}$ turning angle used by Gannon (2002) and the $31.5^{\circ}$ turning angle used by Kirstein (2001). These two points are indicated in Figure A-9. 


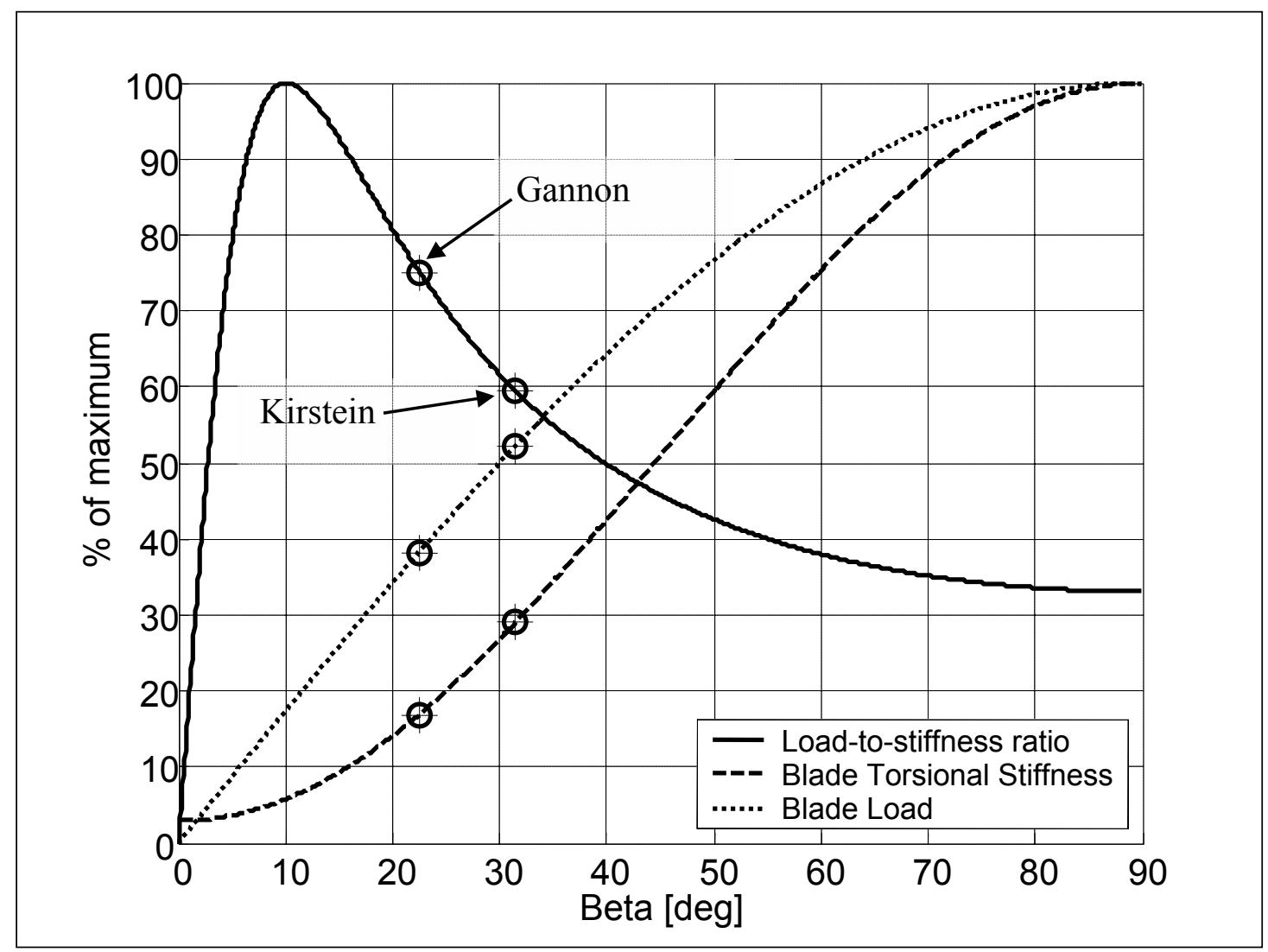

Figure A-9 - Load-to-stiffness ratio of the IGV cascade

The results from increasing the blade angle are:

- the load-to-stiffness ratio is decreased by $20.8 \%$

- the torsional stiffness of the cascade is increased by $72.4 \%$

- the tangential load on the blades is increased by $36.5 \%$

These results indicate that it is desirable to increase the blade angle beyond $10^{\circ}$ as much as possible for structural strength. The ratio can also be decreased below $10^{\circ}$ for structural strength, but then the IGVs are not delivering much swirl to the turbine. Kirstein (2001) determined that better efficiencies are obtainable with this greater flow angle, now it is seen that the structural strength is also improved significantly. 


\section{A.4 Derivations of Equations}

\section{A.4.1 SPECIFIC SPEEDS}

The power specific speed is defined by Sayers (1990) to be

$$
N_{S P}=N \cdot P^{1 / 2} / \rho^{1 / 2}(g H)^{5 / 4}
$$

where $\mathbf{N}$ is the rotation al speed, $\mathbf{P}$ the power and $\mathbf{H}$ the pressure head. The specific speed conversely is defined as

$$
N_{S}=N \cdot Q^{1 / 2} /(g H)^{3 / 4}
$$

By multiplying equation ( A-39) into ( A-38) the following is obtained

$$
\begin{aligned}
N_{S P} & =N_{S} \frac{(g H)^{3 / 4}}{(g H)^{5 / 4}} \frac{N \cdot P^{1 / 2}}{N \cdot Q^{1 / 2}} \frac{1}{\rho^{1 / 2}} \\
& =N_{S} \frac{1}{(g H)^{1 / 2}} \frac{P^{1 / 2}}{Q^{1 / 2}} \frac{1}{\rho^{1 / 2}}
\end{aligned}
$$

But

$$
P=\rho \cdot g \cdot H \cdot Q
$$

$$
\therefore N_{S P}=N_{S}
$$

\section{A.4.2 Static AND Total PRESSURES}

The coefficients for pressure are defined by the following equations (Strohmaier, 1997):

$$
\begin{gathered}
\bar{P}=\frac{P_{2}+P_{3}+P_{4}+P_{5}}{4} \\
C p_{\text {centre }}=\frac{P_{1}-P_{s}}{P_{\text {tot }}-P_{s}} \\
C p_{\text {average }}=\frac{\bar{P}-P_{s}}{P_{\text {tot }}-P_{s}}
\end{gathered}
$$




$$
\begin{gathered}
C p_{Y A W}=\frac{P_{2}-P_{3}}{P_{1}-\bar{P}} \\
C p_{\text {PITCH }}=\frac{P_{4}-P_{5}}{P_{1}-\bar{P}}
\end{gathered}
$$

In order to get the static and total pressures from these equations, equations ( A-42) and ( A-43) is used. Since their denominators are equal we can write

$$
\frac{P_{1}-P_{s}}{C p_{\text {centre }}}=\frac{\bar{P}-P_{s}}{C p_{\text {average }}}
$$

If $P_{s}$ is written on the left hand side

$$
P_{s}\left(\frac{C p_{\text {centre }}}{C p_{\text {average }}}-1\right)=\frac{C p_{\text {centre }}}{C p_{\text {average }}} \bar{P}-P_{1}
$$

By multiplying throughout by $\frac{C p_{\text {average }}}{C p_{\text {centre }}-C p_{\text {average }}}$ then yields

$$
P_{s}=\frac{C p_{\text {centre }} \cdot \bar{P}-C p_{\text {average }} \cdot P_{1}}{C p_{\text {centre }}-C p_{\text {average }}}
$$

Now by using equation ( $\mathrm{A}-42$ ) the equation for $P_{t}$ can be rewritten as

$$
P_{t}=P_{s}+\frac{P_{1}-P_{s}}{C p_{\text {centre }}}
$$

since $P_{s}$ is determined from equation ( A-48).

\section{A.4.3 ERROR ESTIMATION STATISTICS}

Let the average of $n$ samples be $\bar{X}_{n} . \bar{X}_{n}$ can then be written as 


$$
\bar{X}_{n}=\frac{\sum_{i=1}^{n} x_{i}}{n}
$$

where $x_{i}$ is the value of sample number $i$. Now we can write that for $n+1$ samples

$$
\bar{X}_{n+1}=\frac{\sum_{i=1}^{n+1} x_{i}}{n+1}
$$

This can be deconstructed to

$$
\bar{X}_{n+1}=\frac{\sum_{i=1}^{n} x_{i}+x_{n+1}}{n+1}
$$

By substituting in equation ( A-50) the new average can be written as

$$
\bar{X}_{n+1}=\frac{n \cdot \bar{X}_{n}+x_{n+1}}{n+1}
$$

where $x_{n+1}$ is the new sampled value

Variance for $n$ samples is defined by the following equation (Walpole et al., 1998)

$$
\sigma_{n}^{2}=\frac{\sum_{i=1}^{n}\left(x_{i}-\bar{X}_{n}\right)^{2}}{(n-1) \cdot n}
$$

or for $n+1$ samples as 


$$
\sigma_{n+1}^{2}=\frac{\sum_{i=1}^{n+1}\left(x_{i}-\bar{X}_{n+1}\right)^{2}}{n(n+1)}
$$

Assuming that $\bar{X}_{n} \approx \bar{X}_{n+1}$, which is reasonable for more than 5 samples with as low variance as the cases in this project, equation ( A-55) can be rewritten as.

$$
\sigma_{n+1}^{2}=\frac{\sum_{i=1}^{n}\left(x_{i}-\bar{X}_{n}\right)^{2}+\left(x_{n+1}-\bar{X}_{n+1}\right)^{2}}{n(n+1)}
$$

By substituting equation ( A-54) into ( A-56) the variance for the new sample can be written as

$$
\sigma_{n+1}^{2}=\sigma_{n}^{2} \frac{n-1}{n+1}+\frac{\left(x_{n+1}-\bar{X}_{n+1}\right)^{2}}{n(n+1)}
$$

To determine the variance for the coefficients of yaw and pitch the equation of Granger (1988) is used, namely

$$
B_{F}{ }^{2}=\sum_{i=1}^{n}\left(\frac{\partial F}{\partial X_{i}} B_{X_{i}}\right)^{2}
$$

From equations ( A-41) and ( A-44) we find that

$$
\begin{aligned}
& \frac{\partial C p_{y}}{\partial P_{2}}=\frac{1}{P_{1}-\bar{P}} \\
& \frac{\partial C p_{y}}{\partial P_{3}}=\frac{-1}{P_{1}-\bar{P}} \\
& \frac{\partial C p_{y}}{\partial P_{1}}=\frac{-\left(P_{2}-P_{3}\right)}{\left(P_{1}-\bar{P}\right)^{2}} \\
& \frac{\partial C p_{y}}{\partial \bar{P}}=\frac{\left(P_{2}-P_{3}\right)}{\left(P_{1}-\bar{P}\right)^{2}}
\end{aligned}
$$


$\therefore \quad B_{C p_{y}}{ }^{2}=\left(\left[\frac{B_{P_{2}}}{P_{1}-\bar{P}}\right]^{2}+\left[\frac{B_{P_{3}}}{P_{1}-\bar{P}}\right]^{2}+\left[-B_{P_{1}} \frac{P_{2}-P_{3}}{\left(P_{1}-\bar{P}\right)^{2}}\right]^{2}+\left[B_{\bar{P}} \frac{P_{2}-P_{3}}{\left(P_{1}-\bar{P}\right)^{2}}\right]^{2}\right)$

But the variances for the pressures as well as their average are equal, therefore

$$
B_{C p_{y}}^{2}=2 B_{P}^{2}\left(\left[\frac{1}{P_{1}-\bar{P}}\right]^{2}+\left[\frac{P_{2}-P_{3}}{\left(P_{1}-\bar{P}\right)^{2}}\right]^{2}\right)
$$

Similarly

$$
B_{C p_{p}}^{2}=2 B_{P}^{2}\left(\left[\frac{1}{P_{1}-\bar{P}}\right]^{2}+\left[\frac{P_{4}-P_{5}}{\left(P_{1}-\bar{P}\right)^{2}}\right]^{2}\right)
$$




\section{APPENDIX B: FIGURES OF RESULTS}

The following results are normalised. The velocities are normalised to the throughflow velocity in a nominal diameter chimney, $V_{c}=Q /\left(D^{2} \pi / 4\right)$; the pressures by the dynamic pressure in a nominal chimney diameter $\mathrm{q}_{\mathrm{c}}=1 / 2 \rho \mathrm{V}_{\mathrm{c}}{ }^{2}$; and the developed distance by the total length of the gap. 

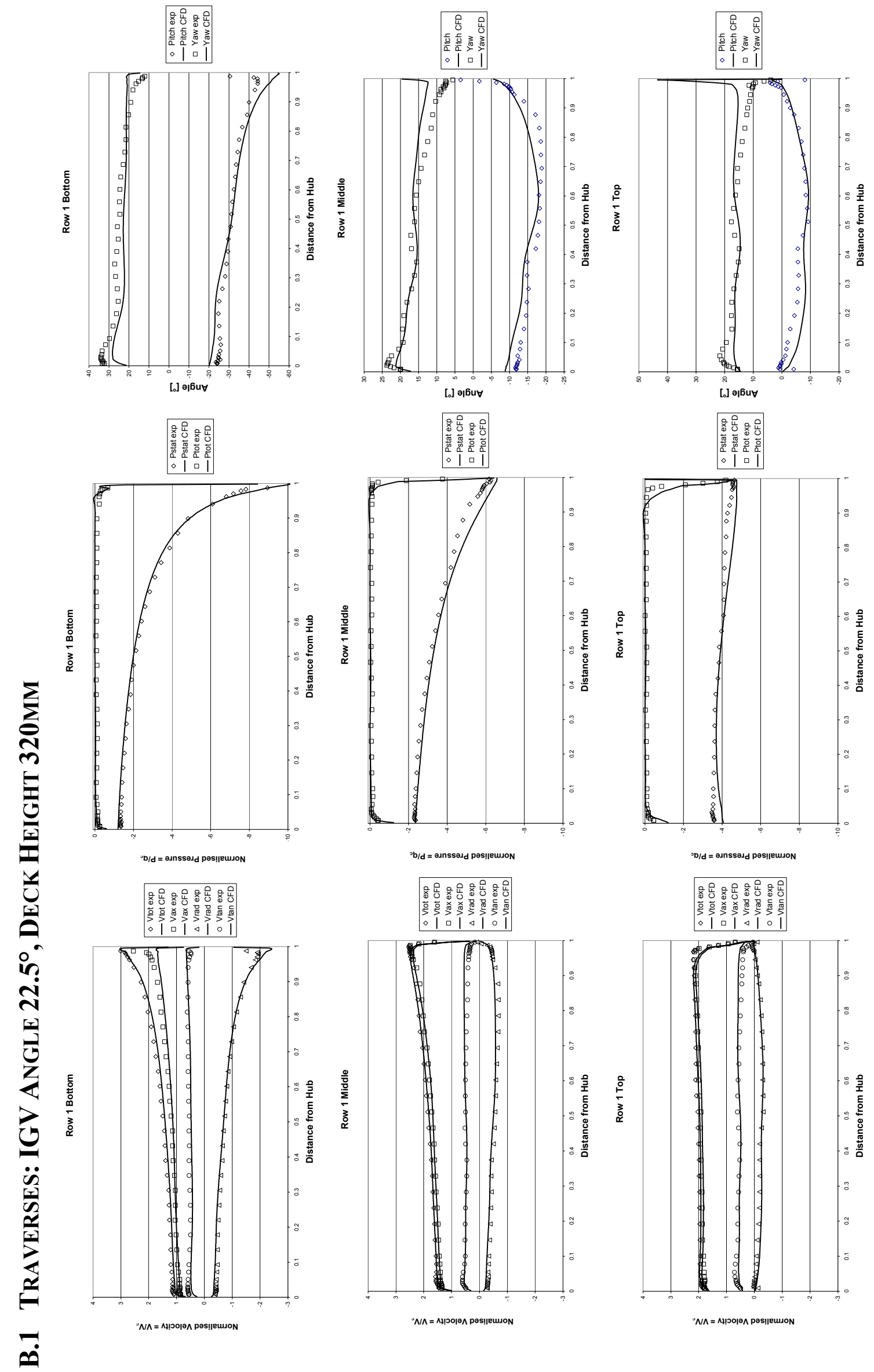

2
$n$

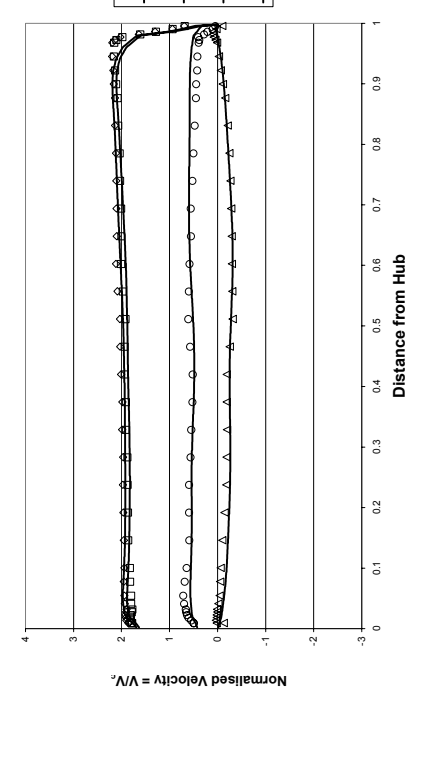

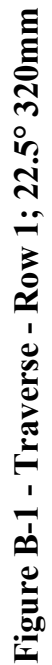



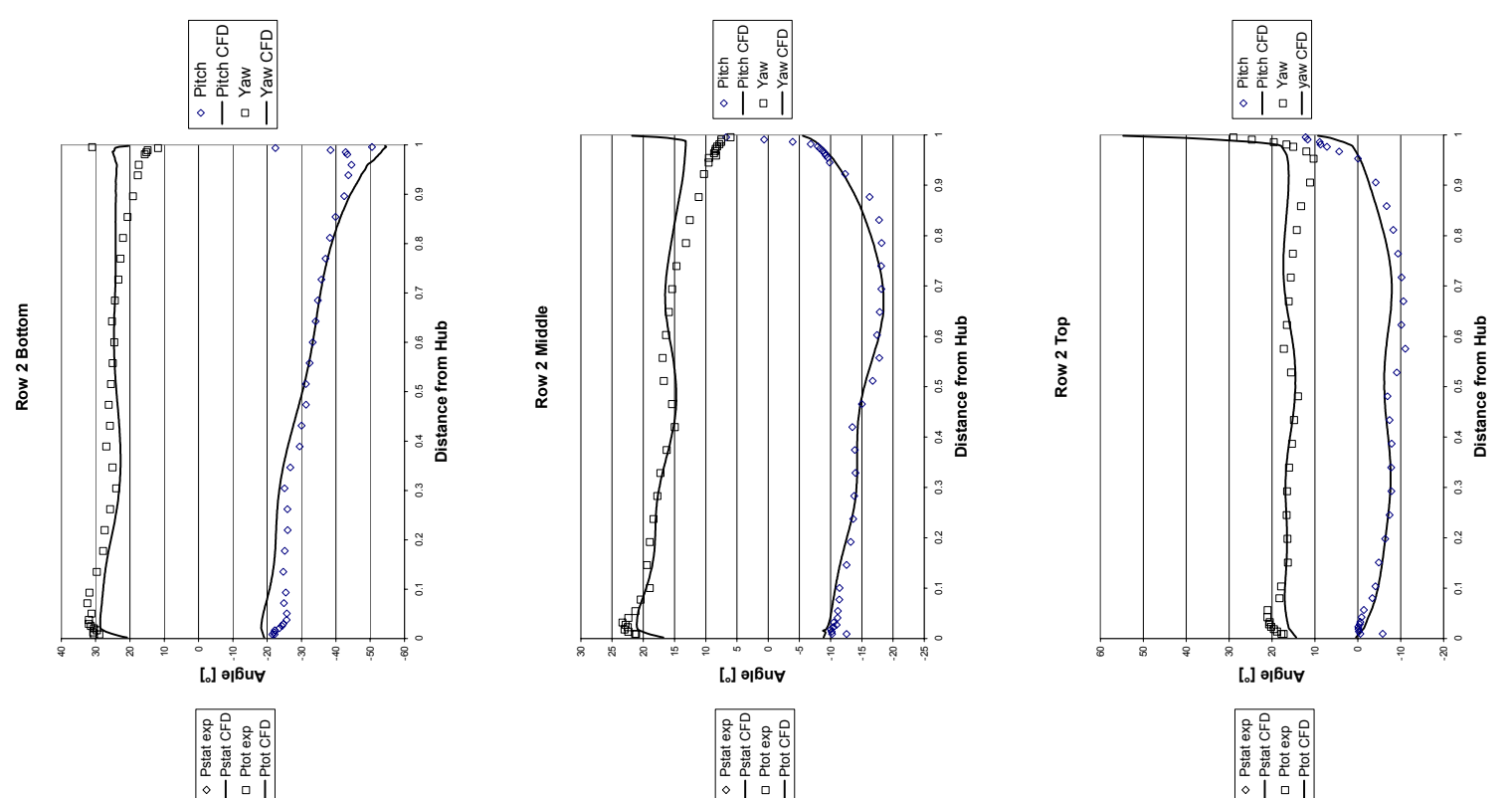

$\stackrel{⿱ 亠 乂}{n}$
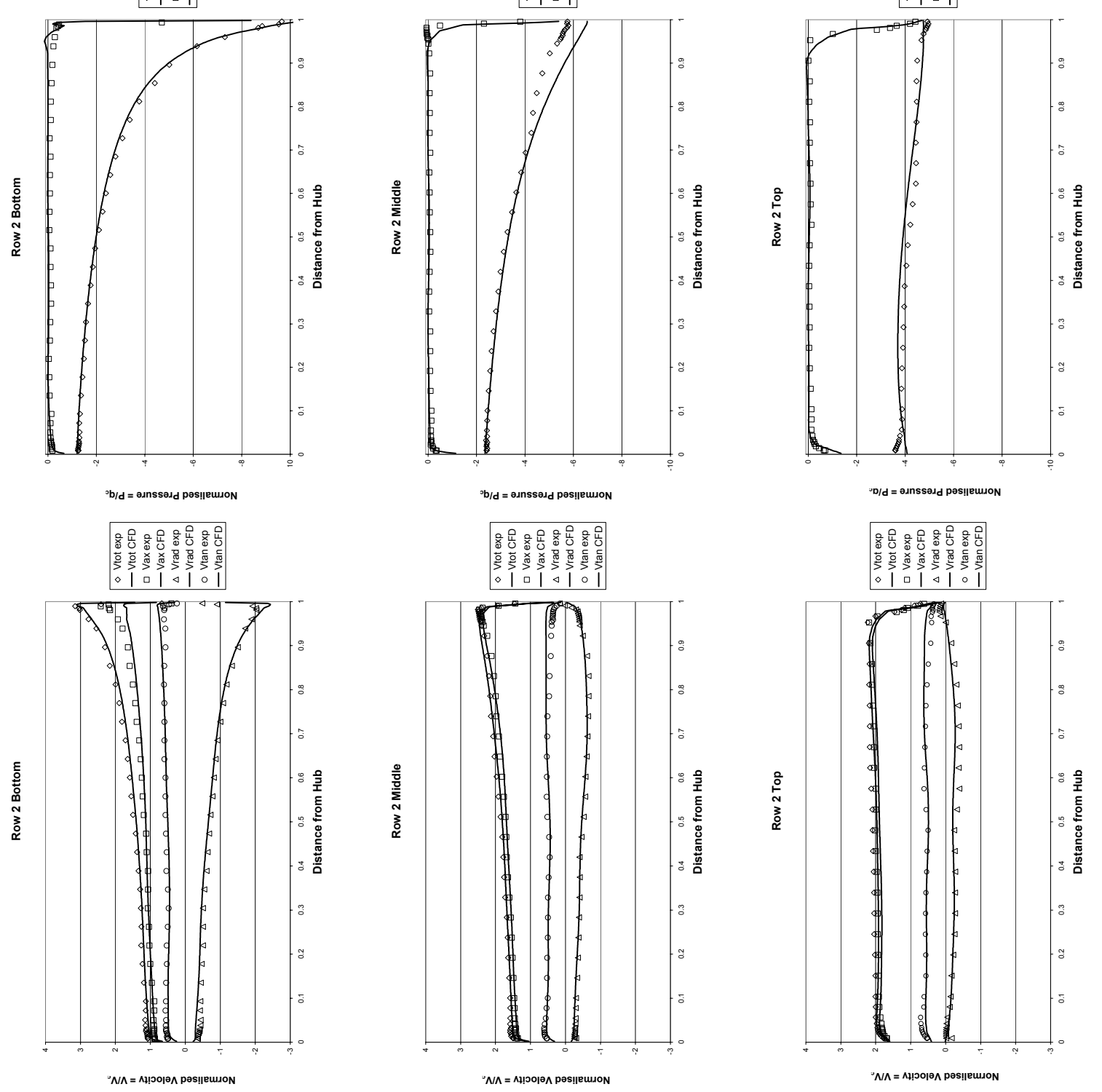

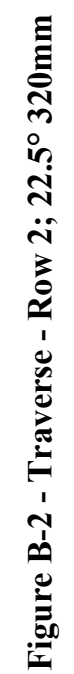



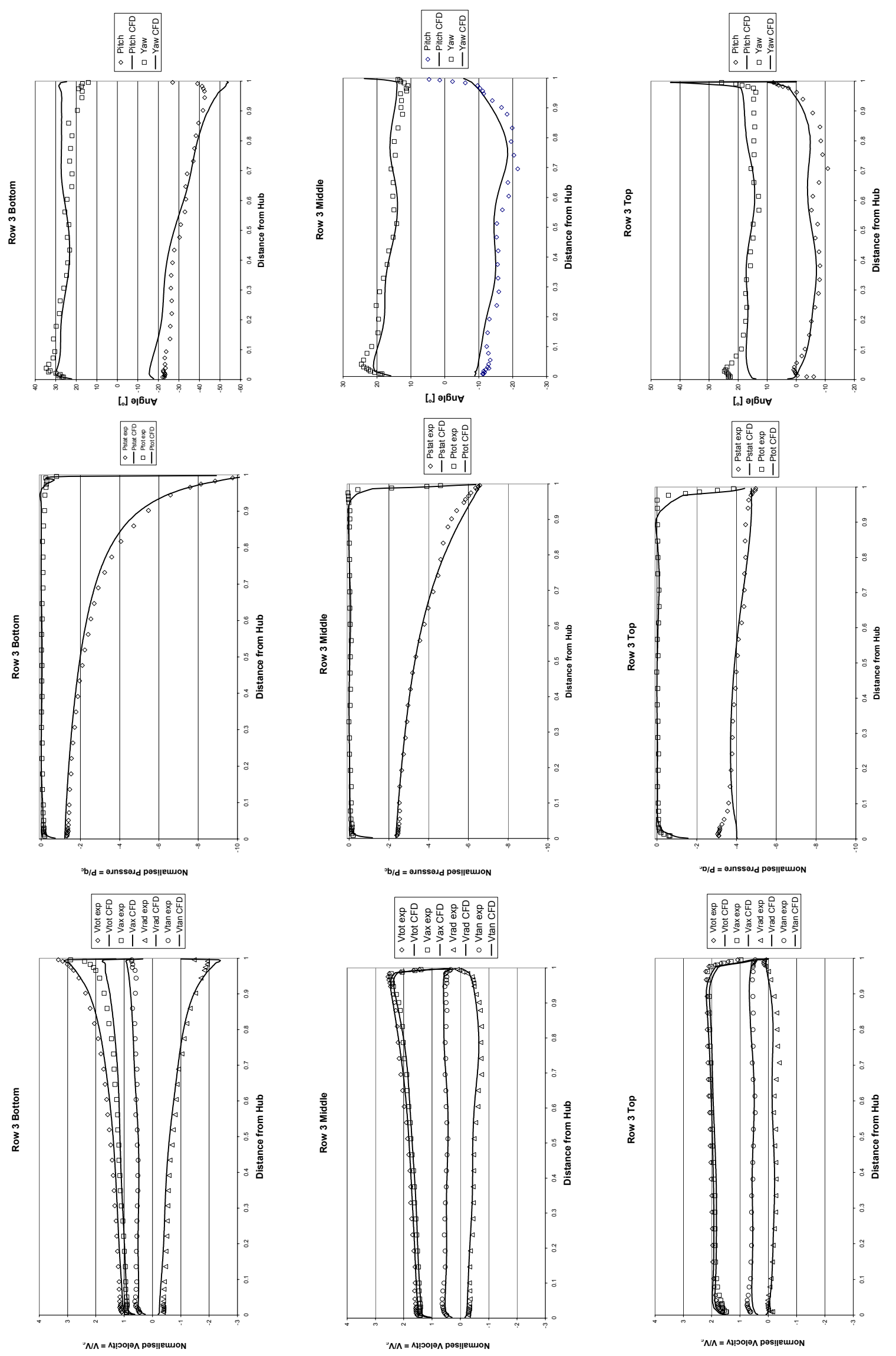

a
$n$

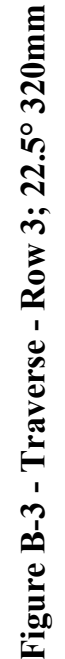




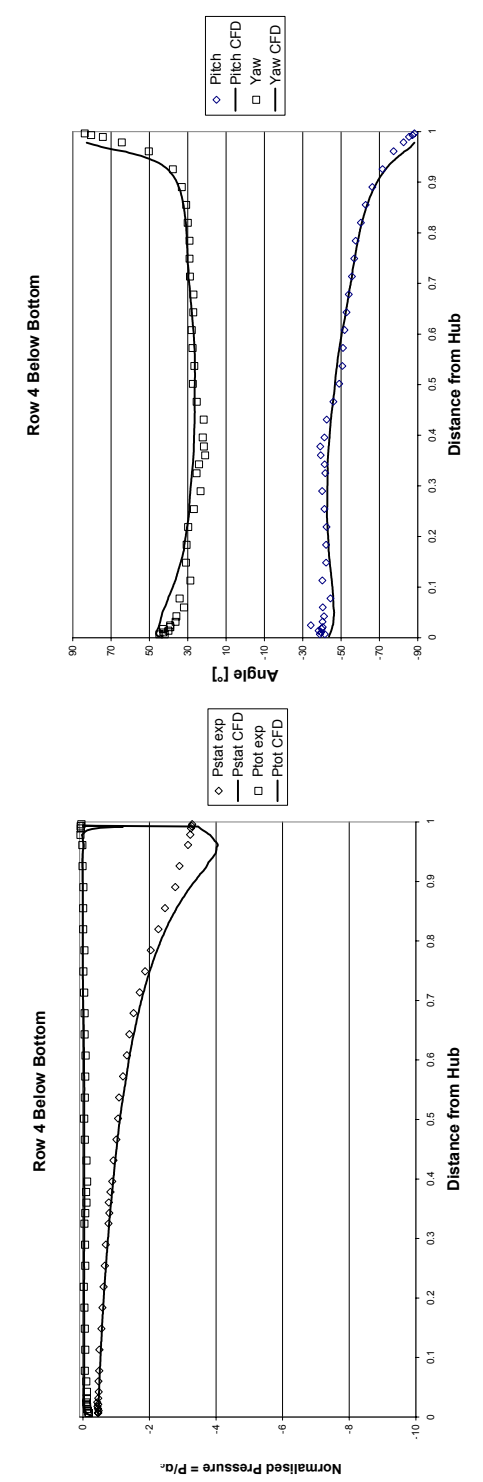

군

ம்

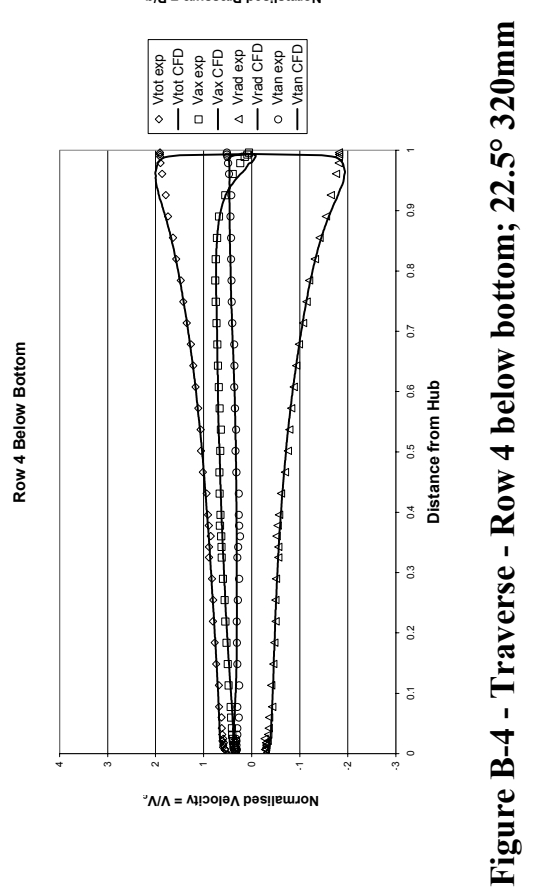



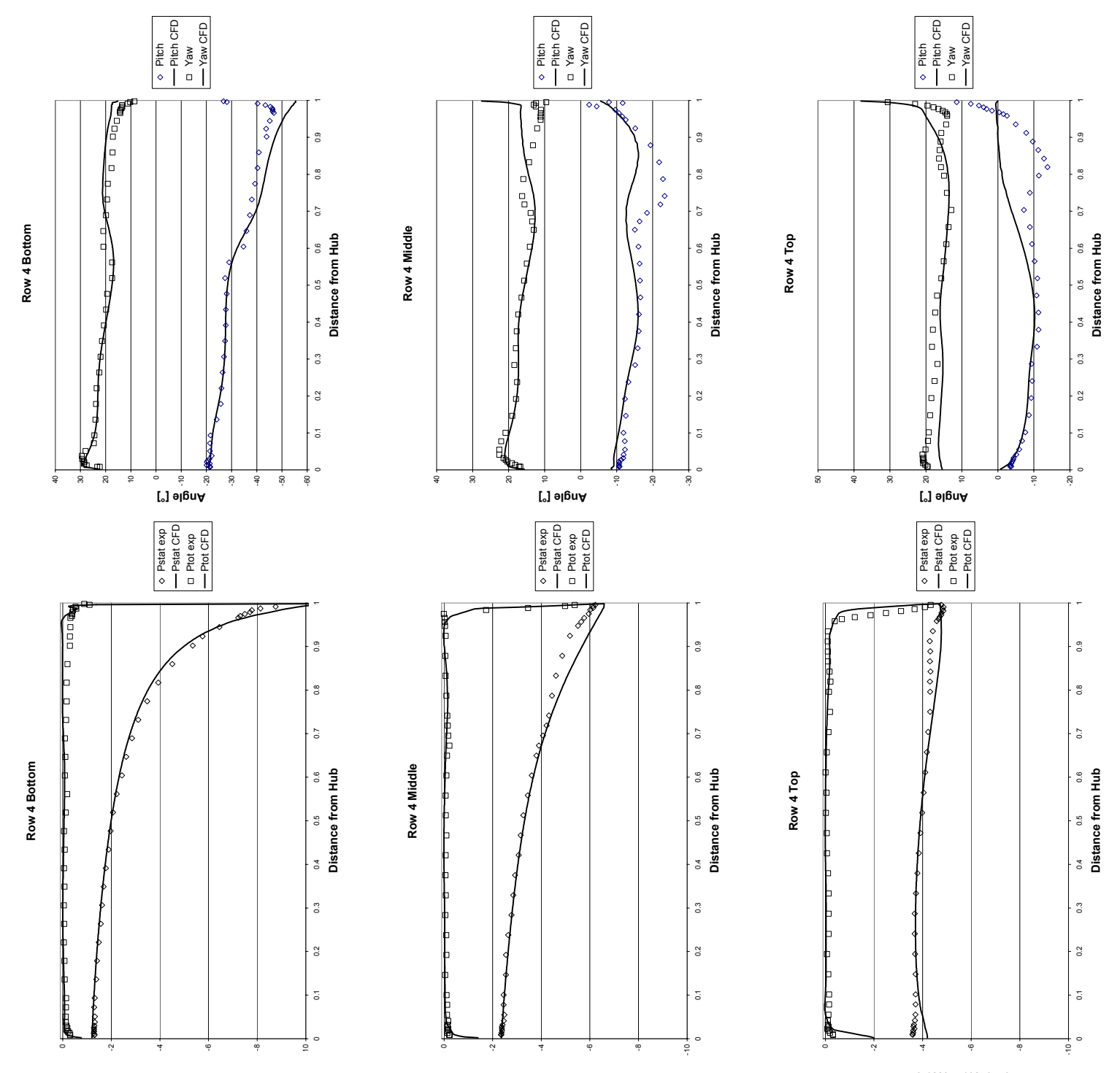

$\hat{N}$
$\dot{n}$
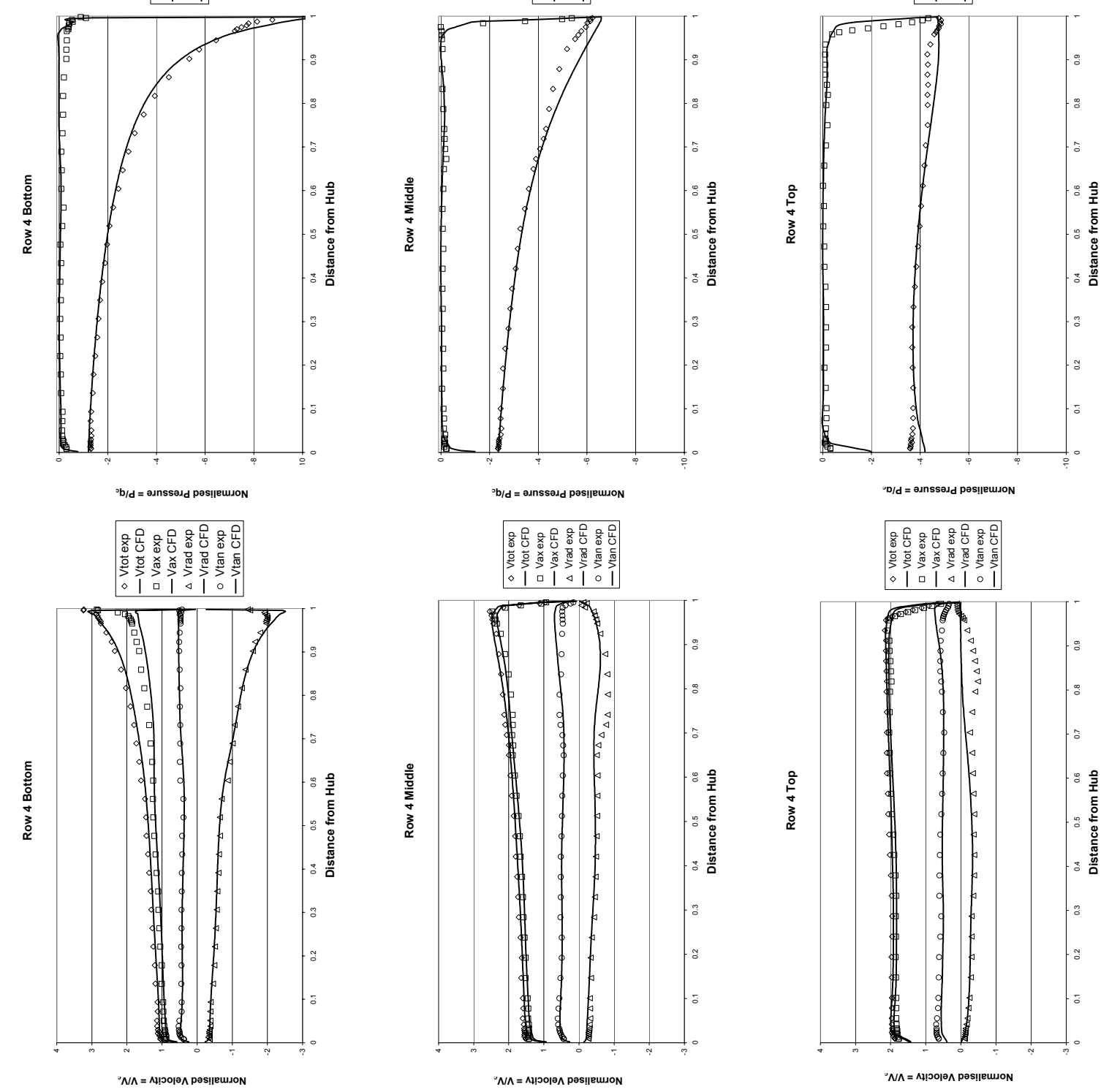

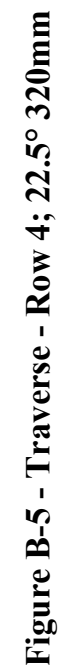



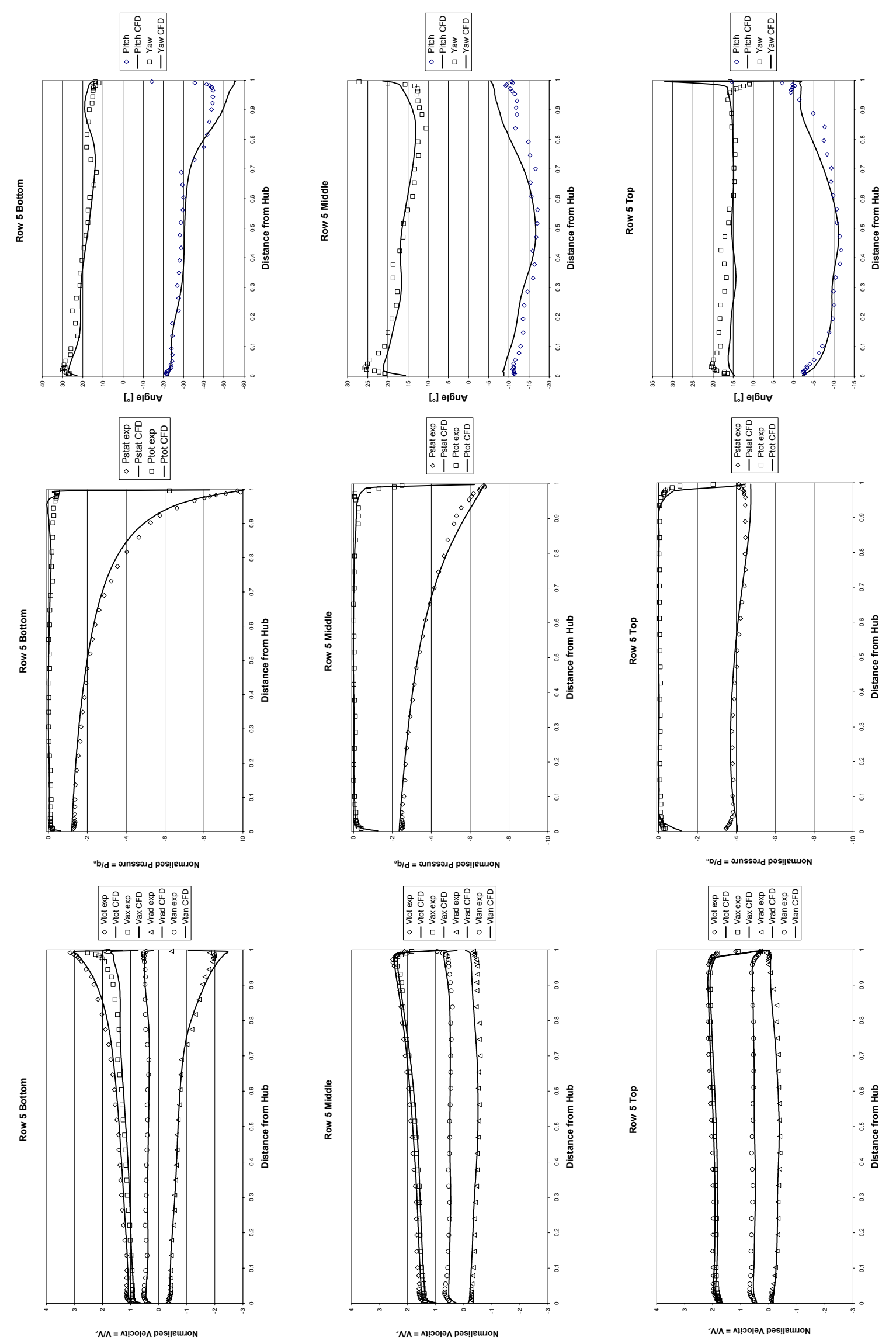

$\stackrel{\infty}{\stackrel{1}{n}}$ 

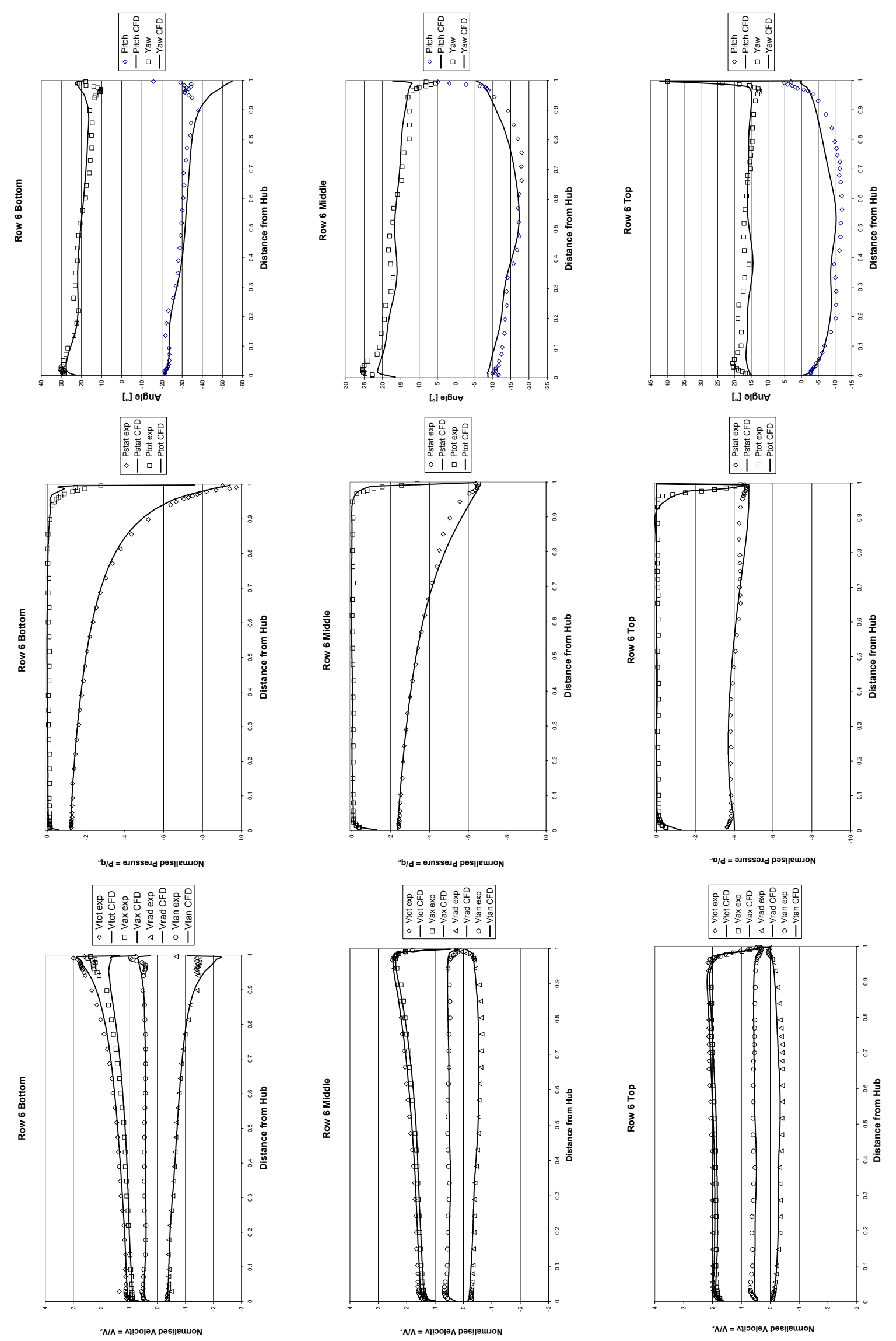

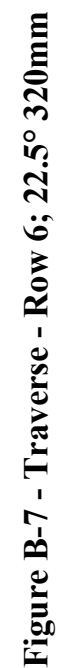



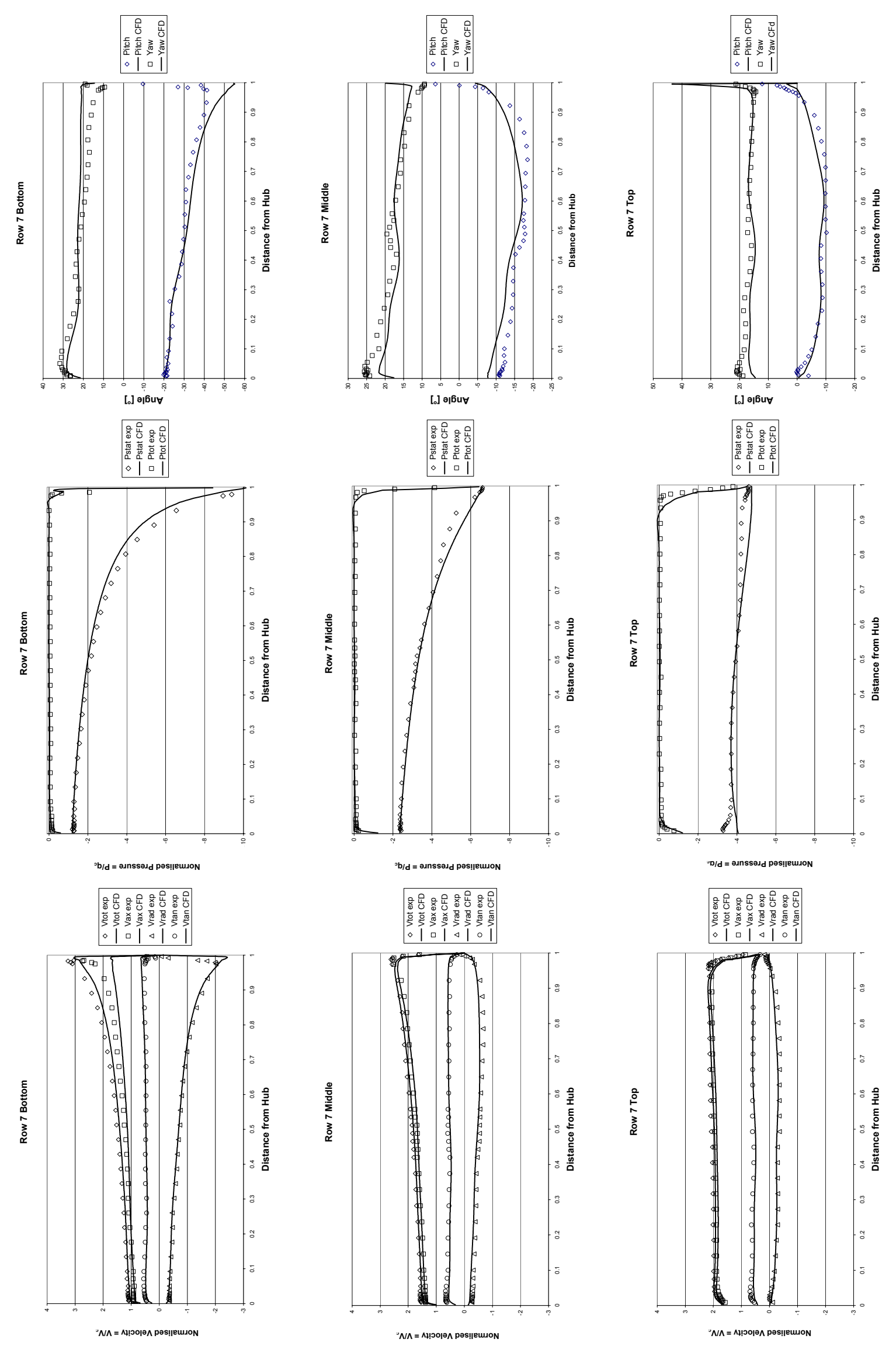

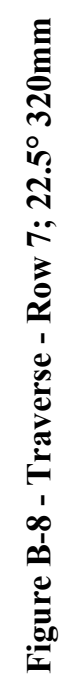



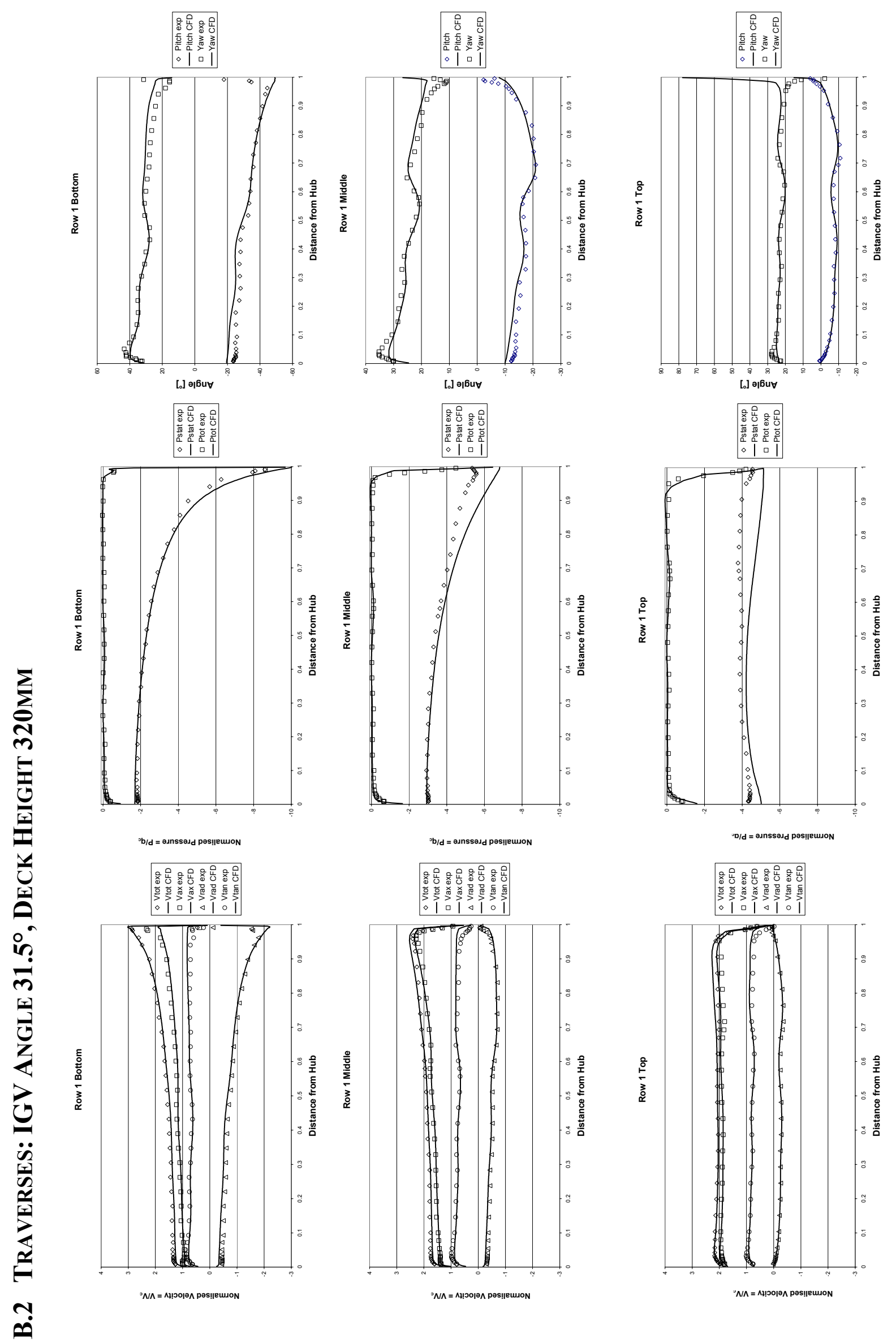

$\vec{m}$
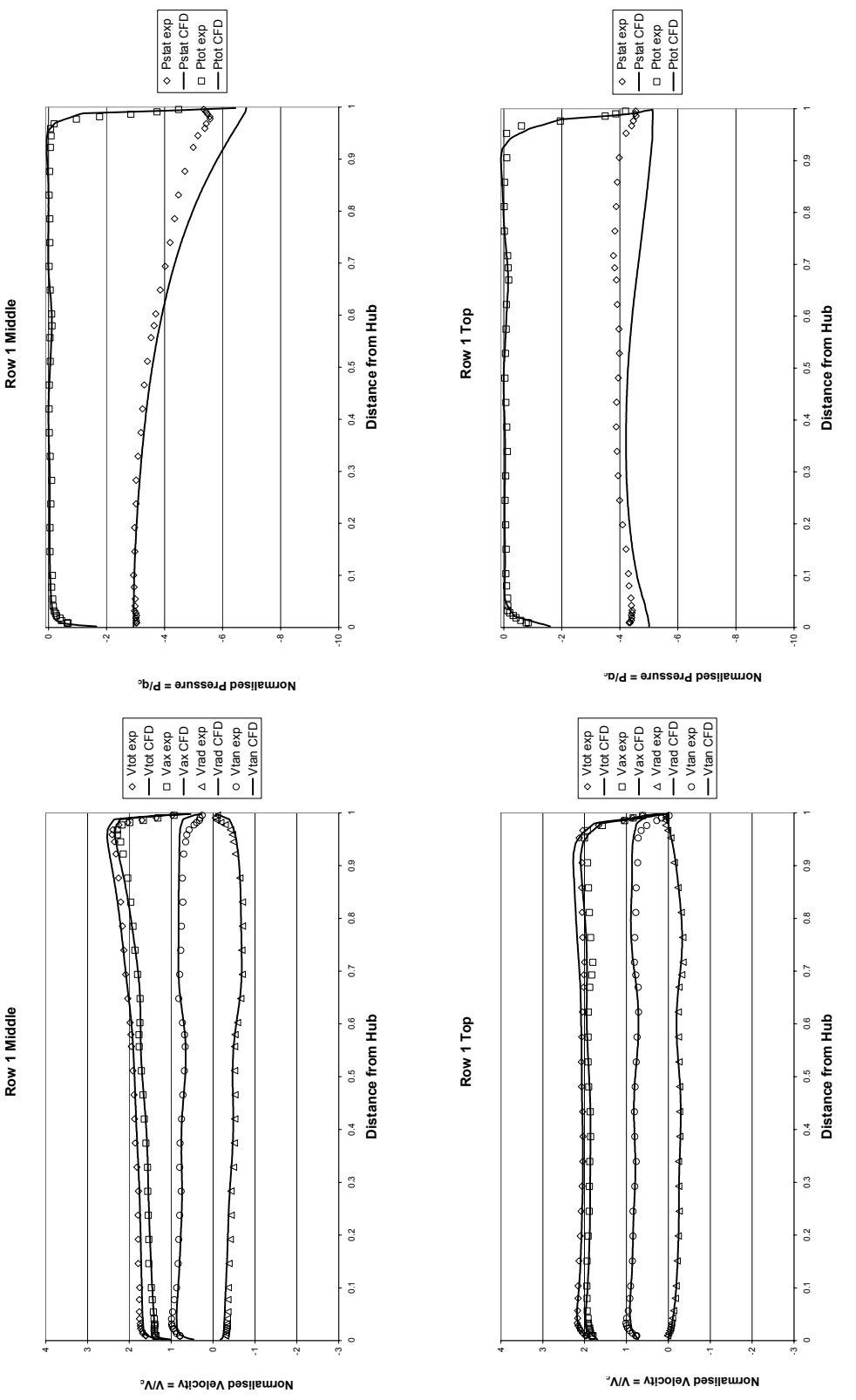

हี 

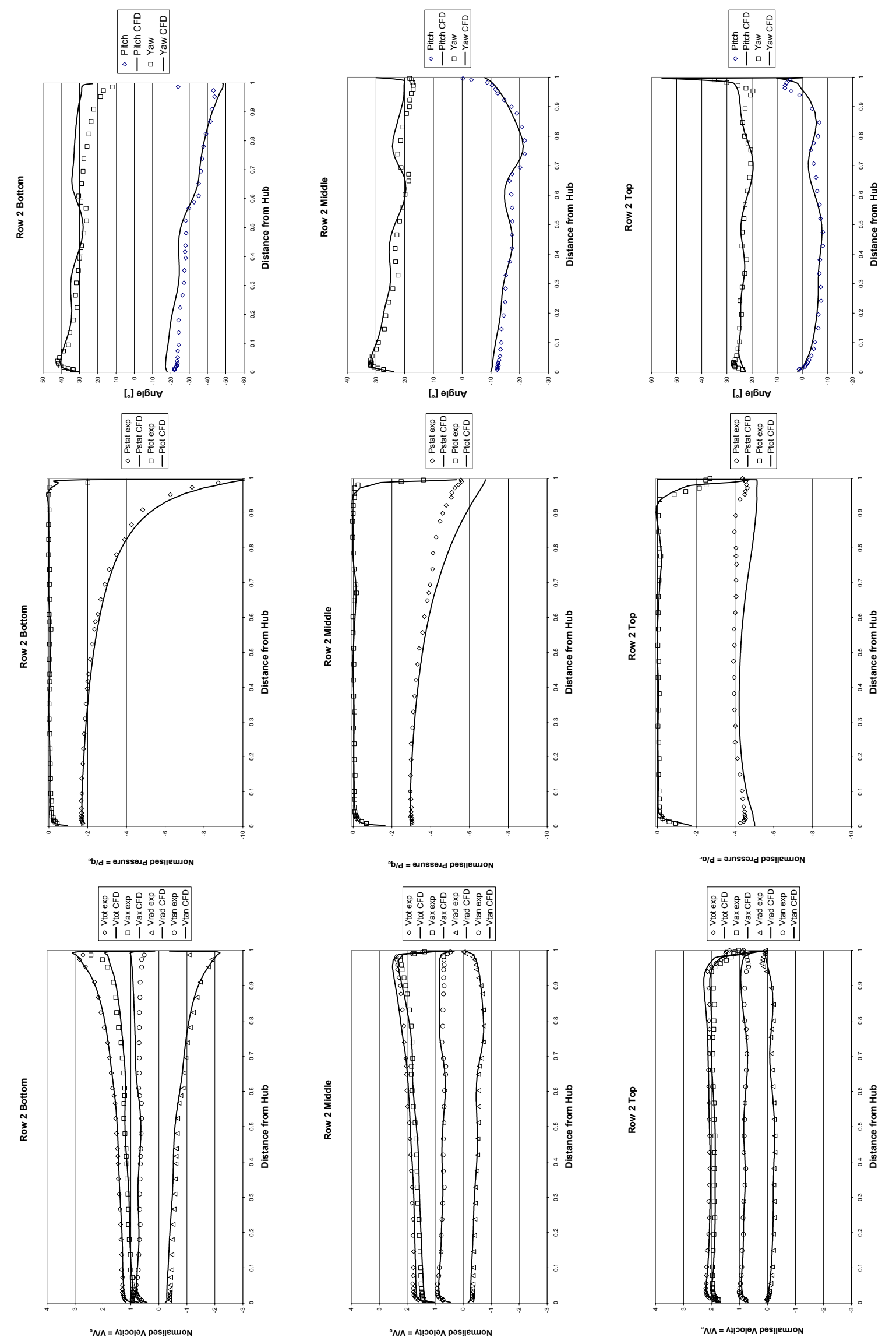

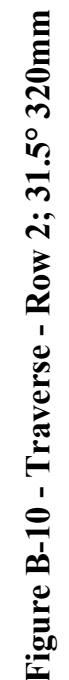



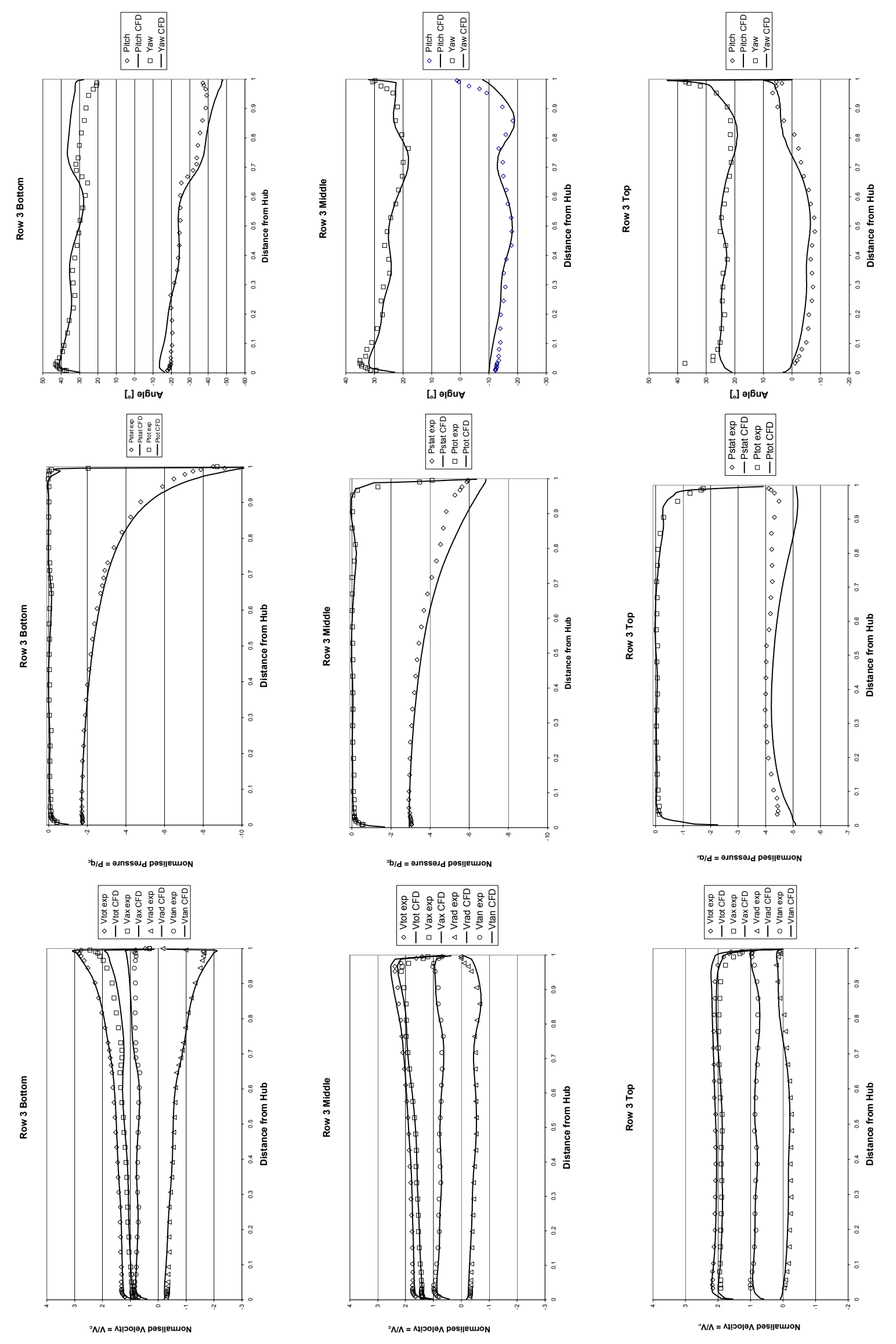

$m$
$m$ 


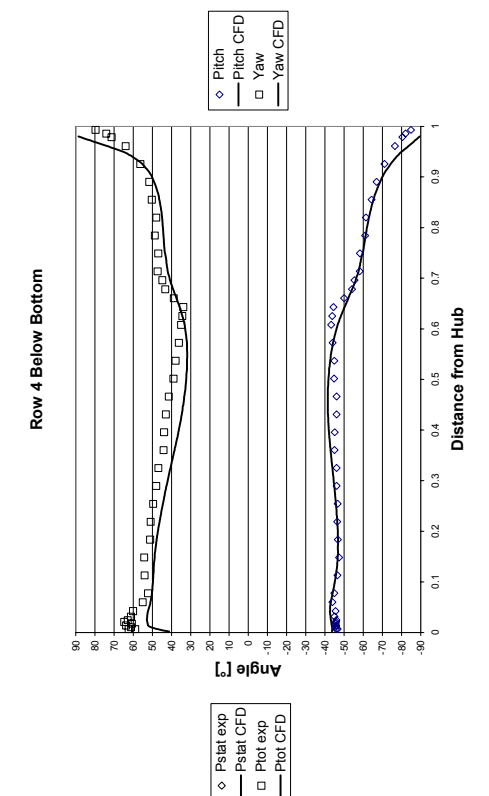

$\dot{m}$
$\dot{m}$
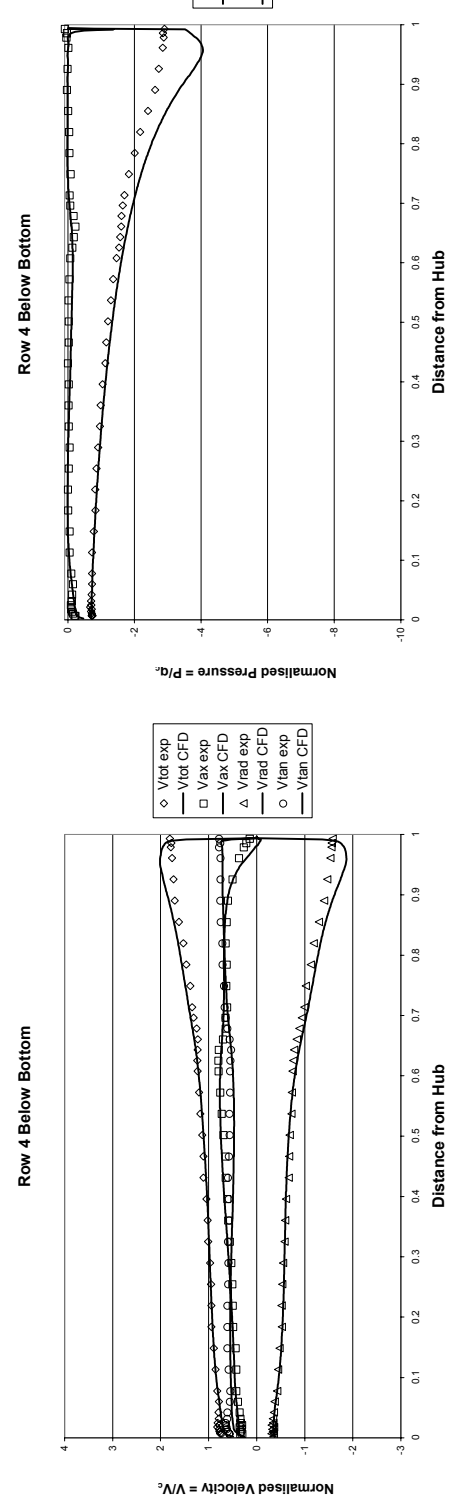

ह⿱艹 

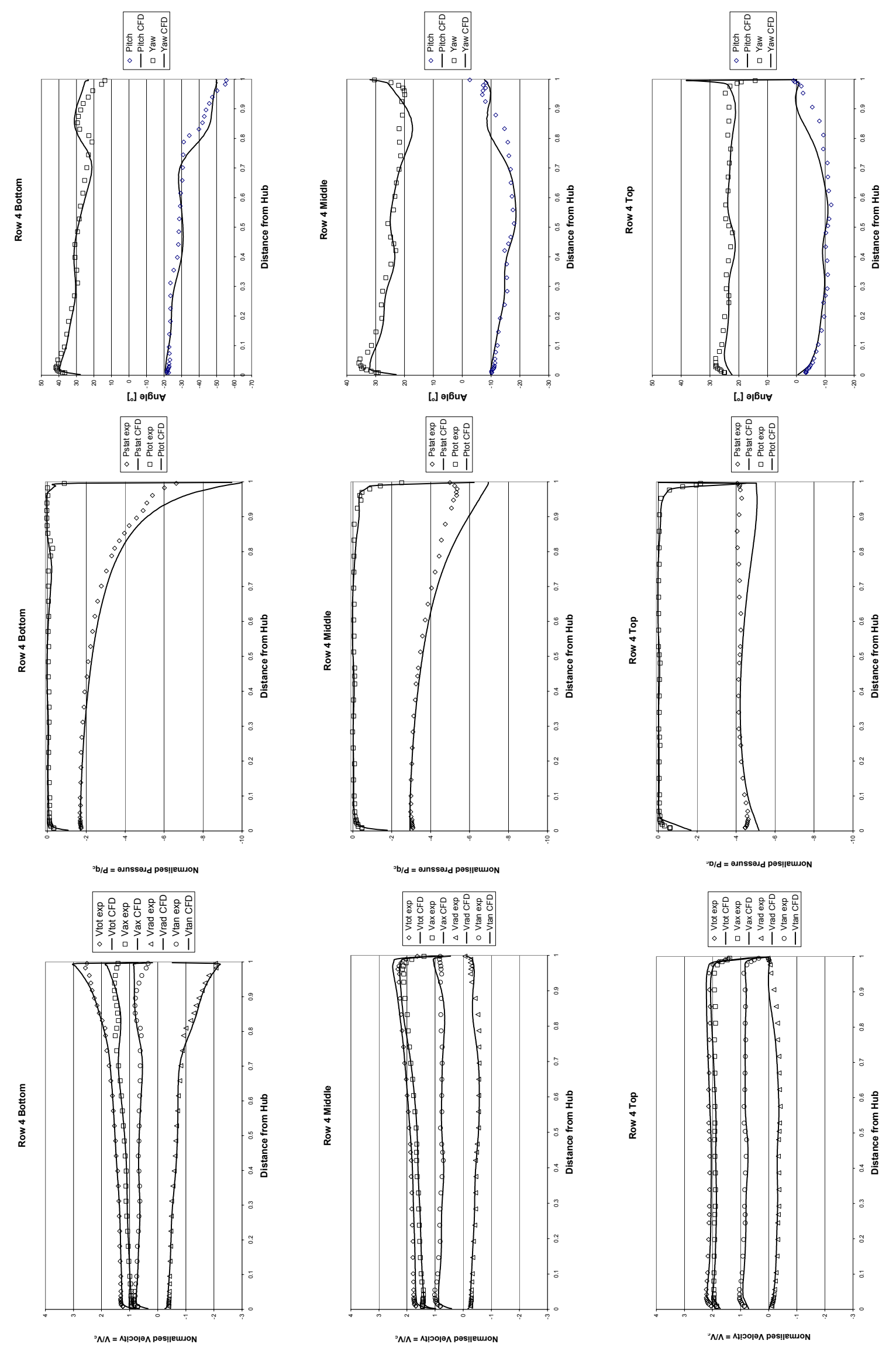

恶 

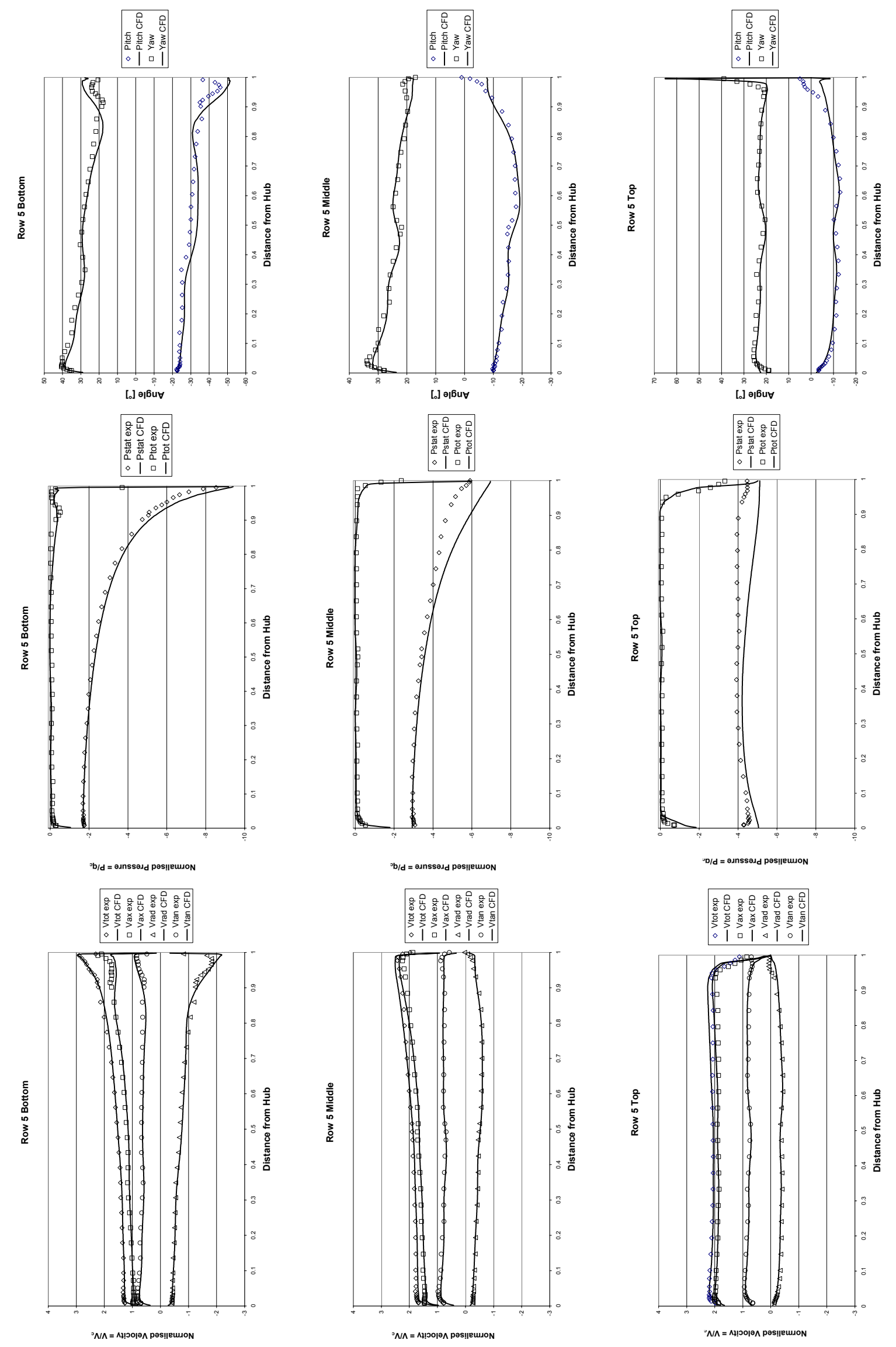

है 

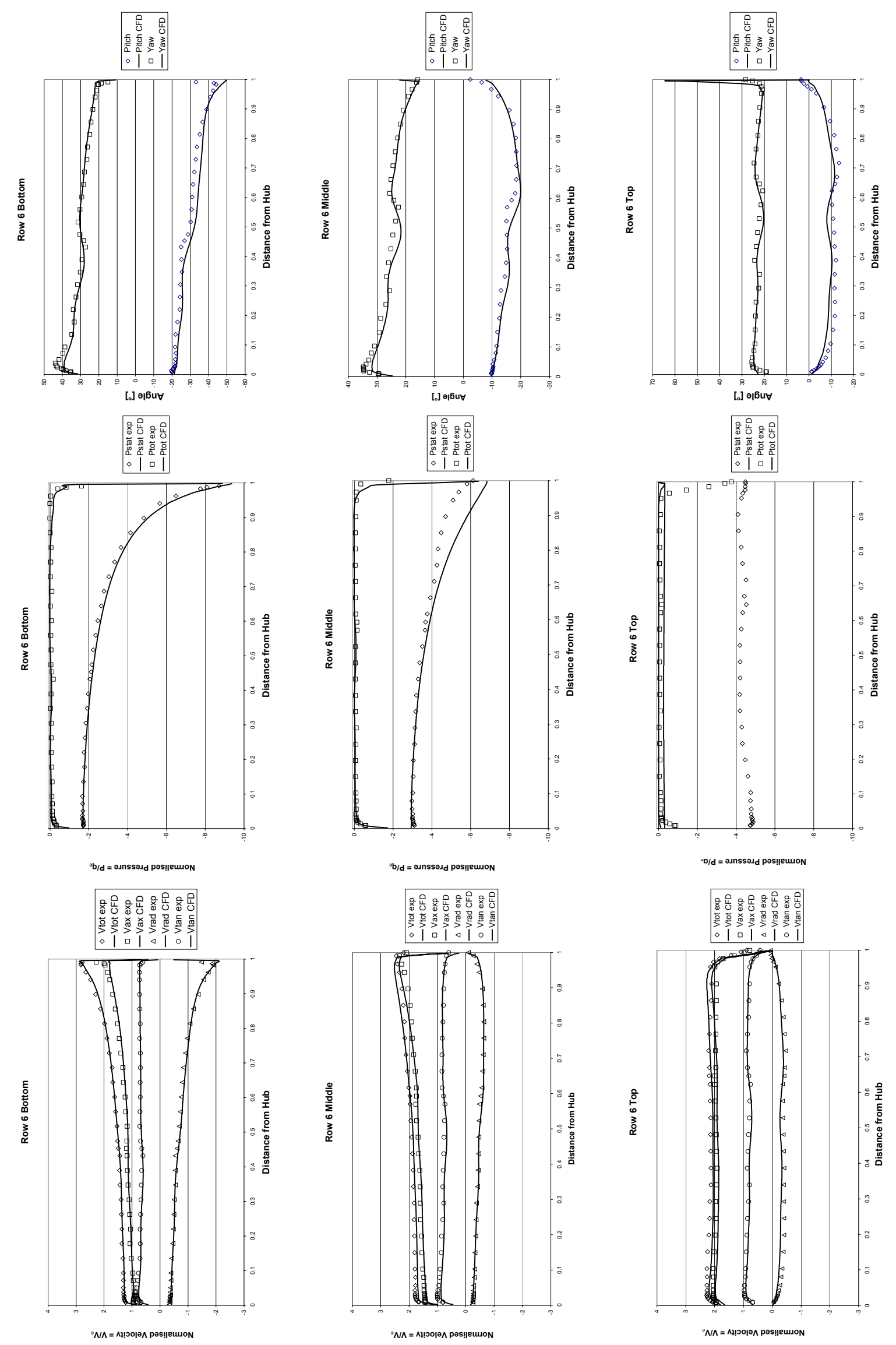

है 

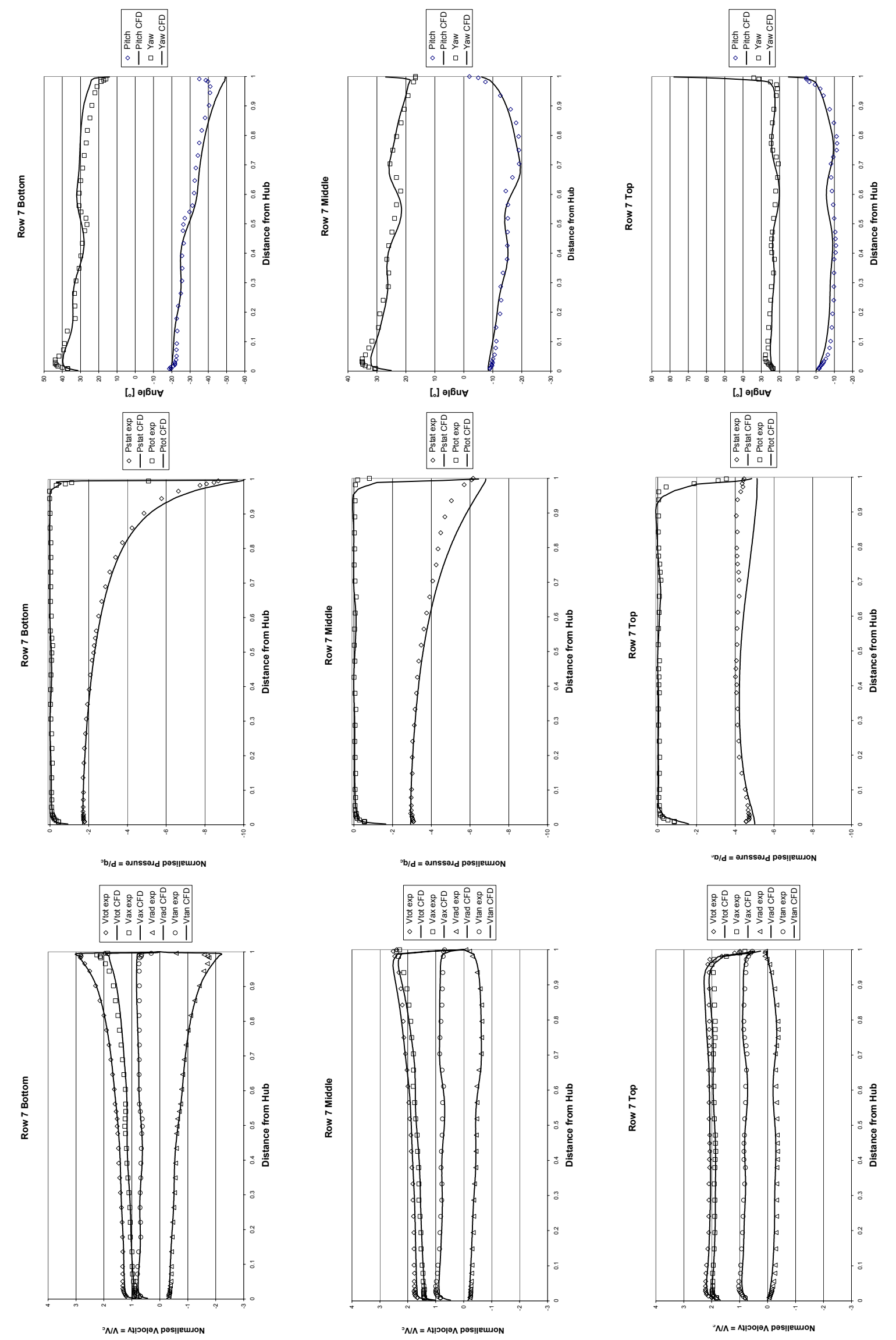

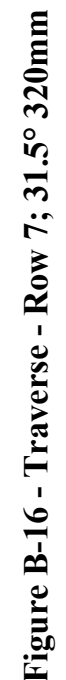




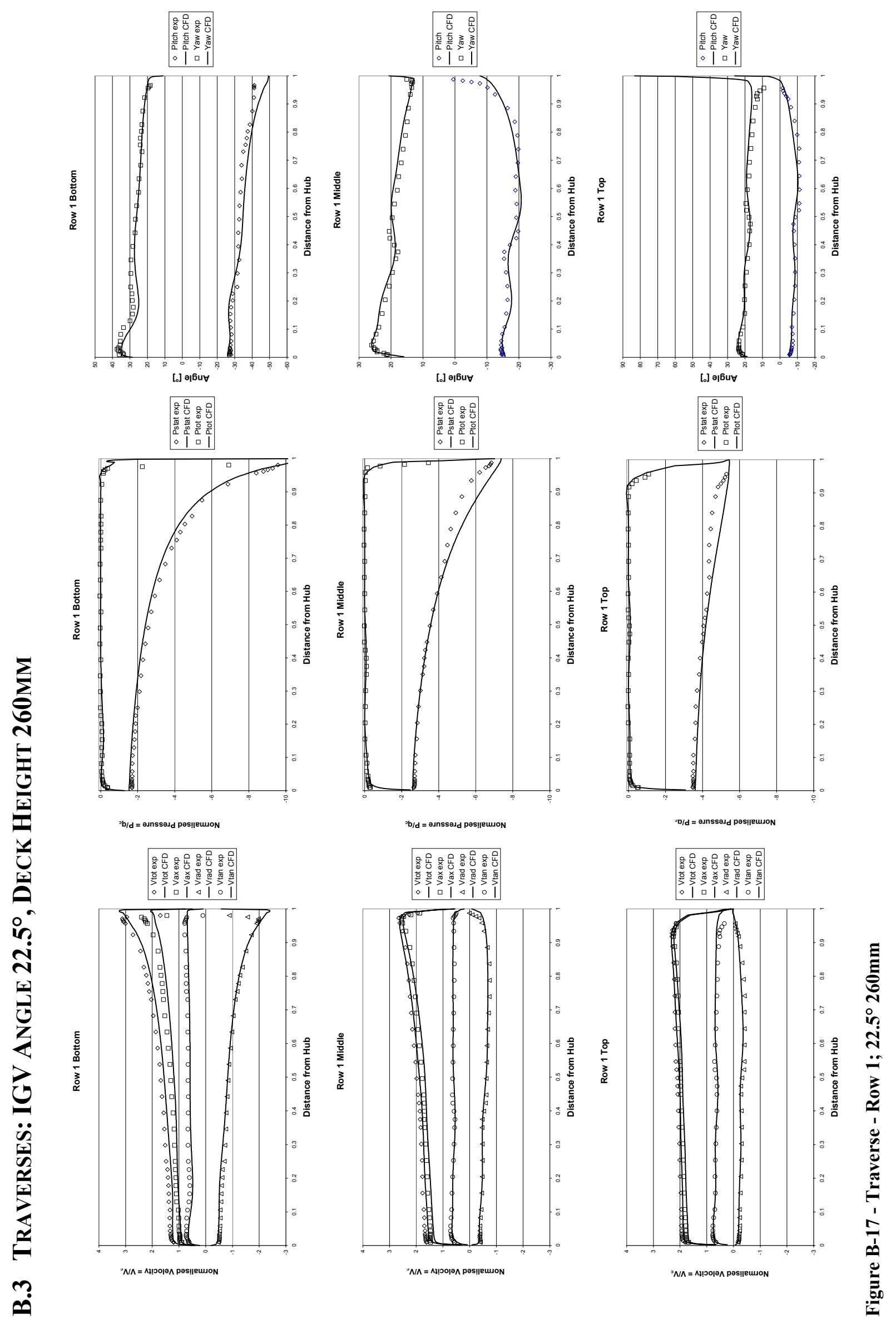



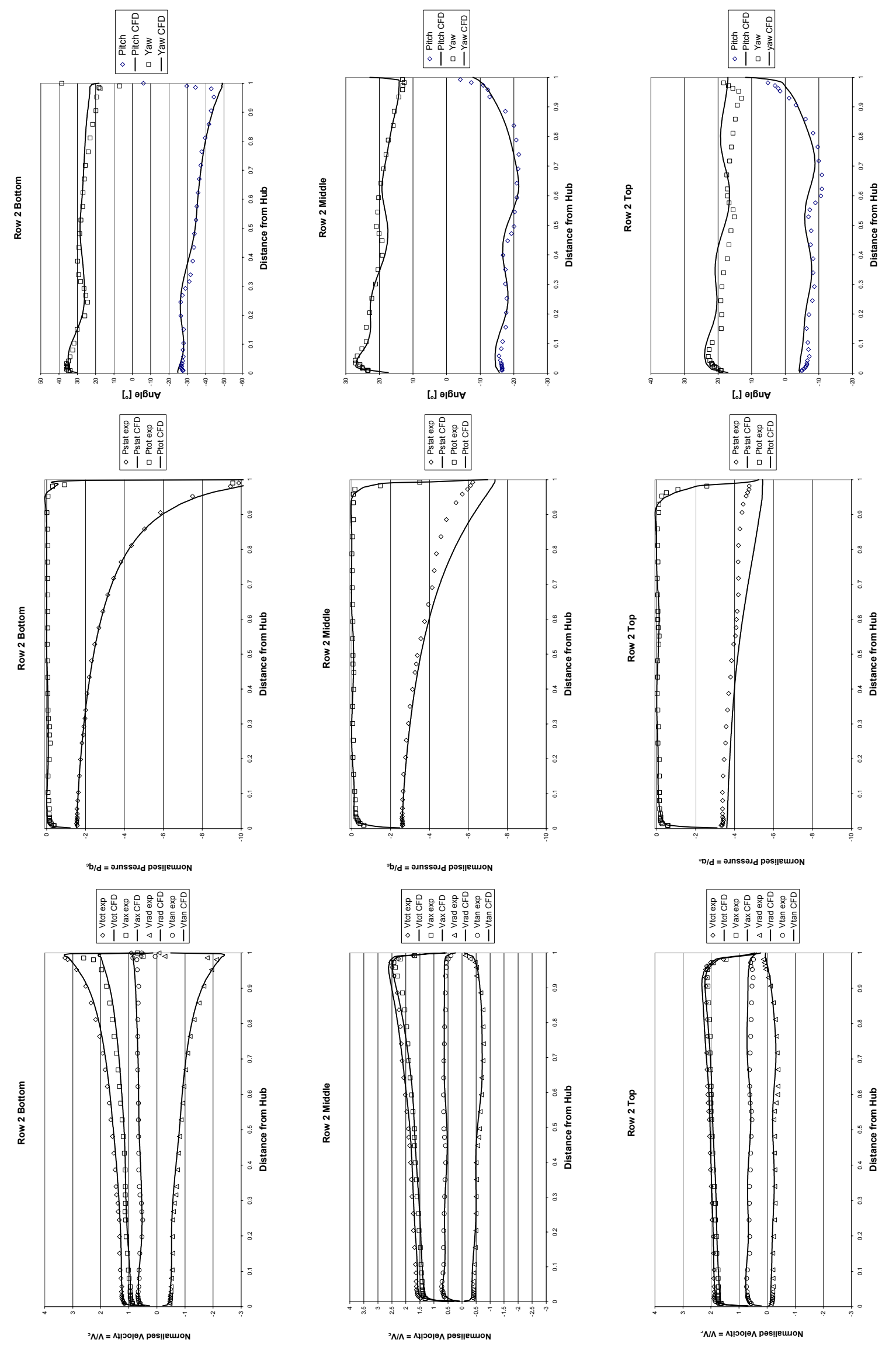

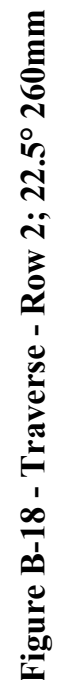



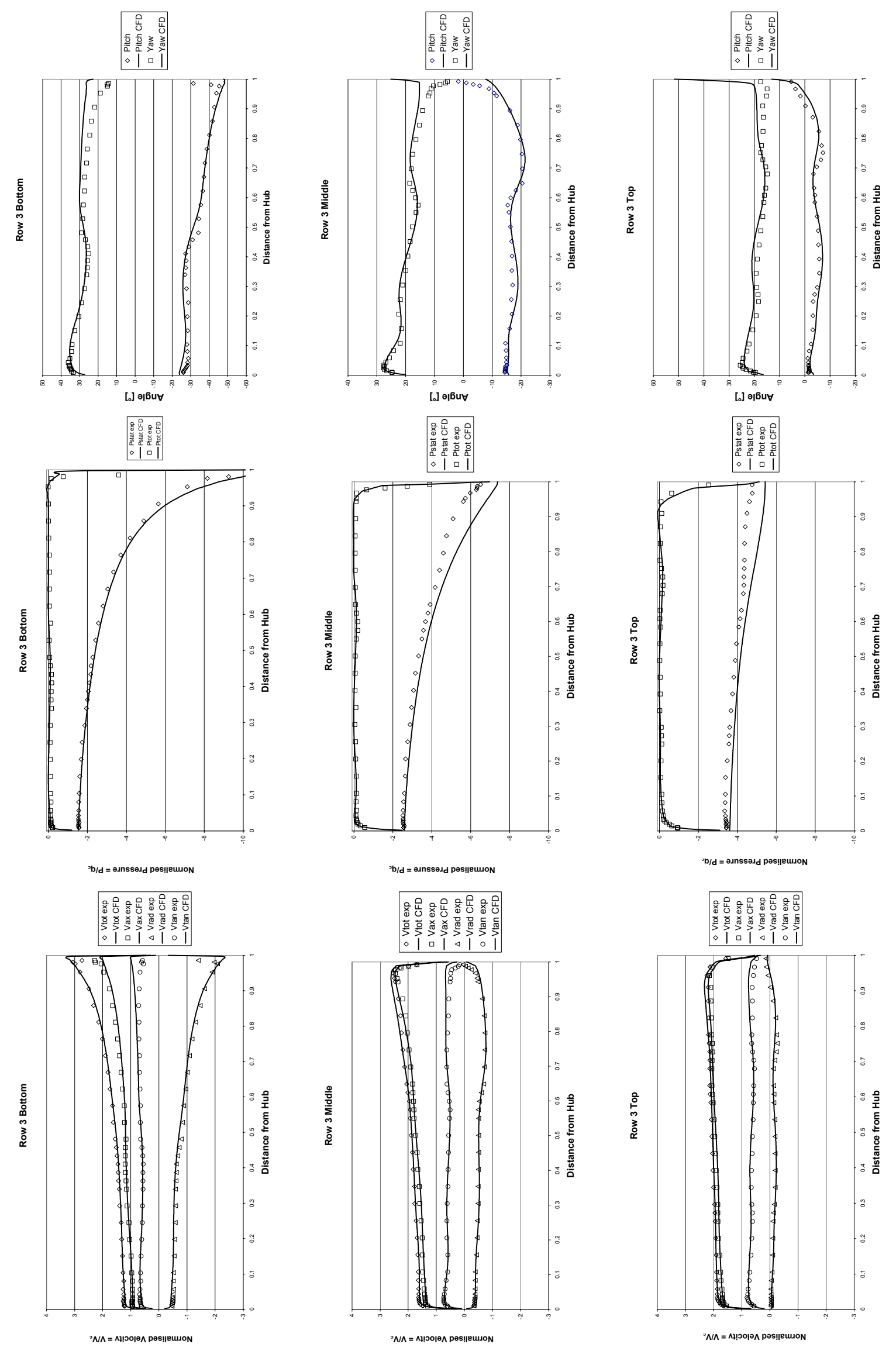

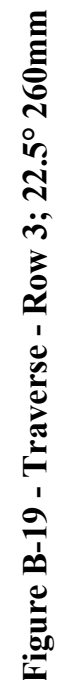




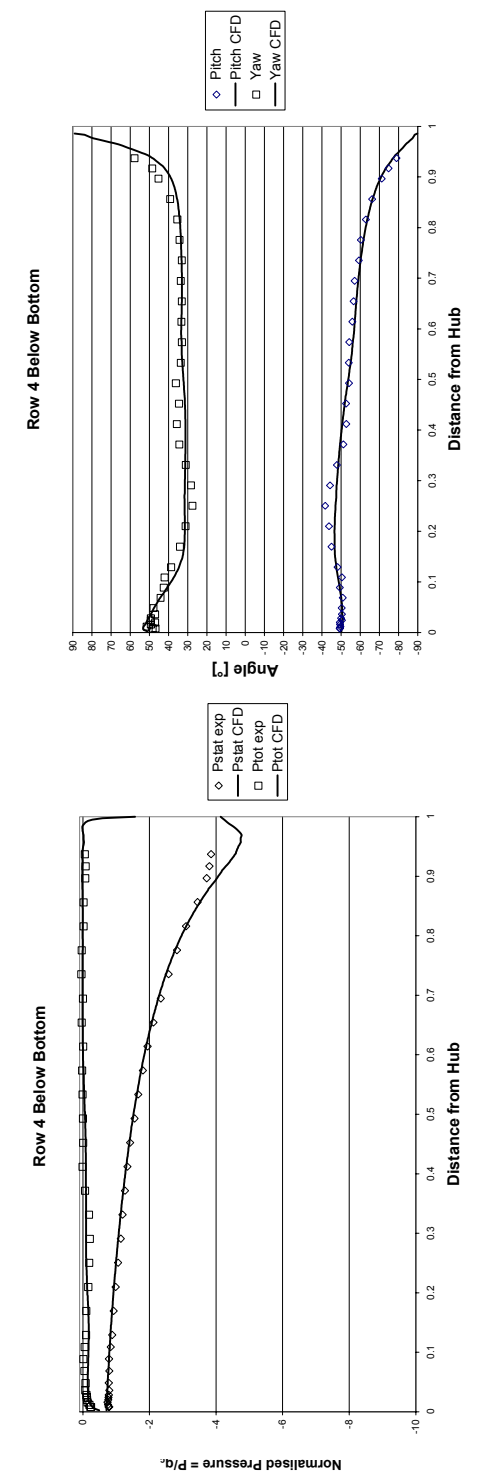

广
๓่

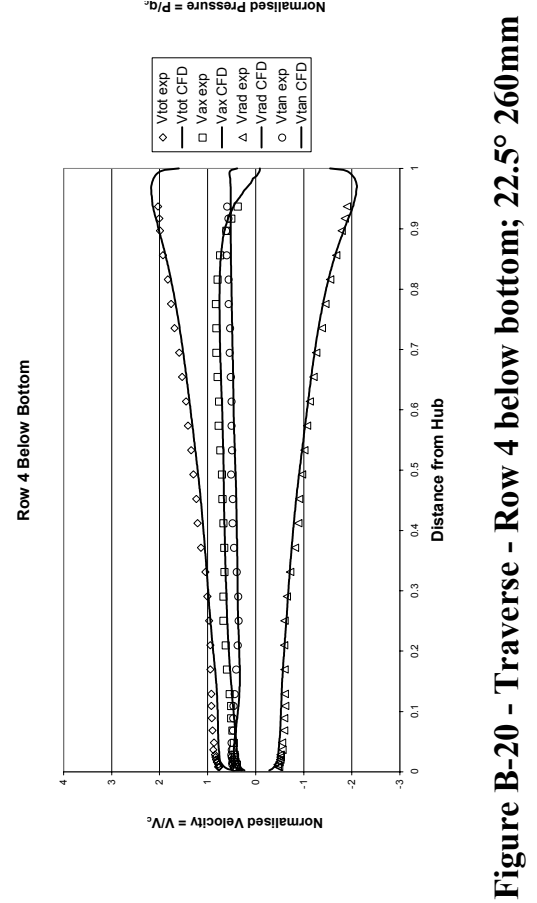



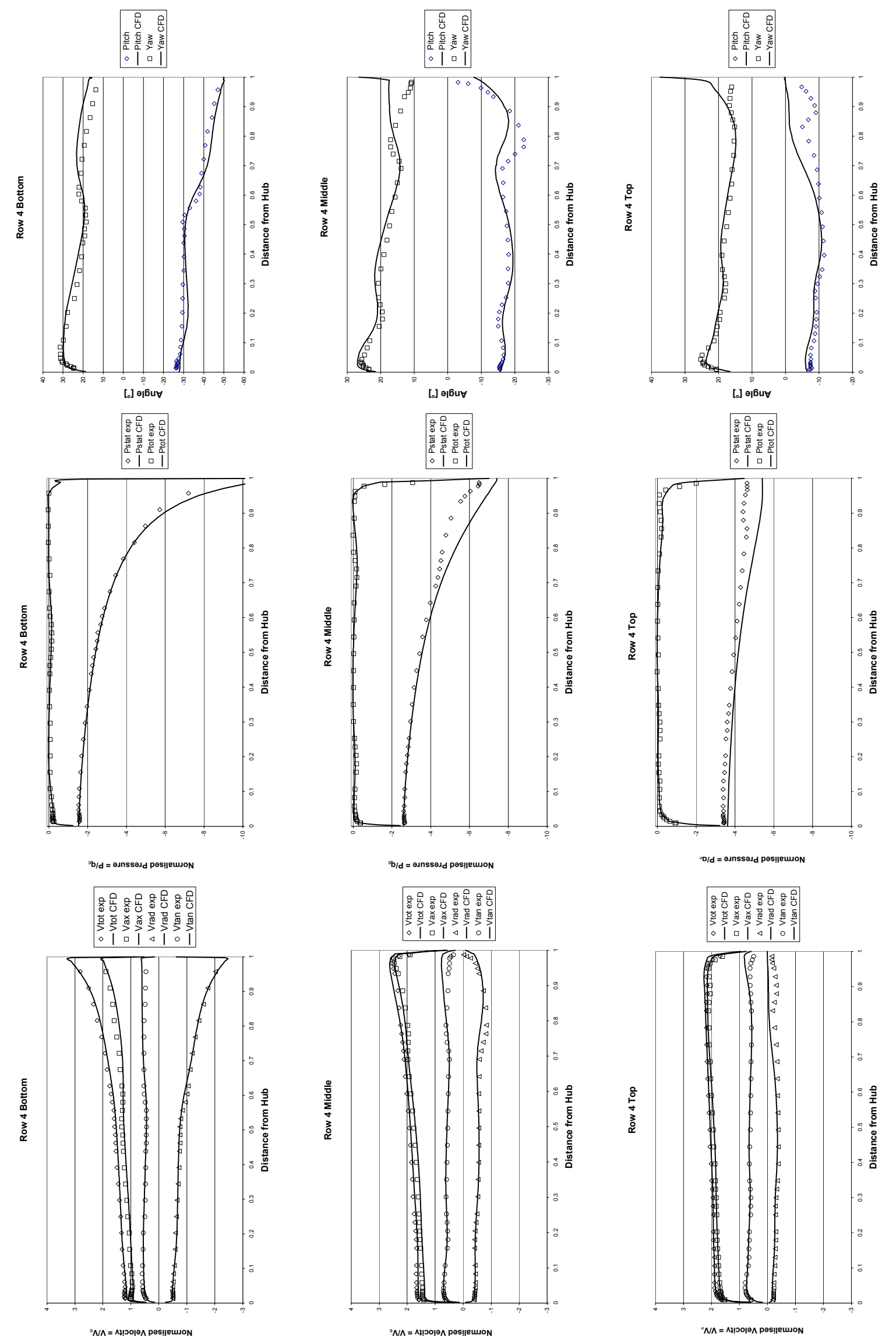

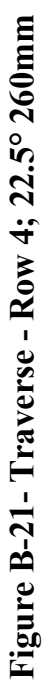



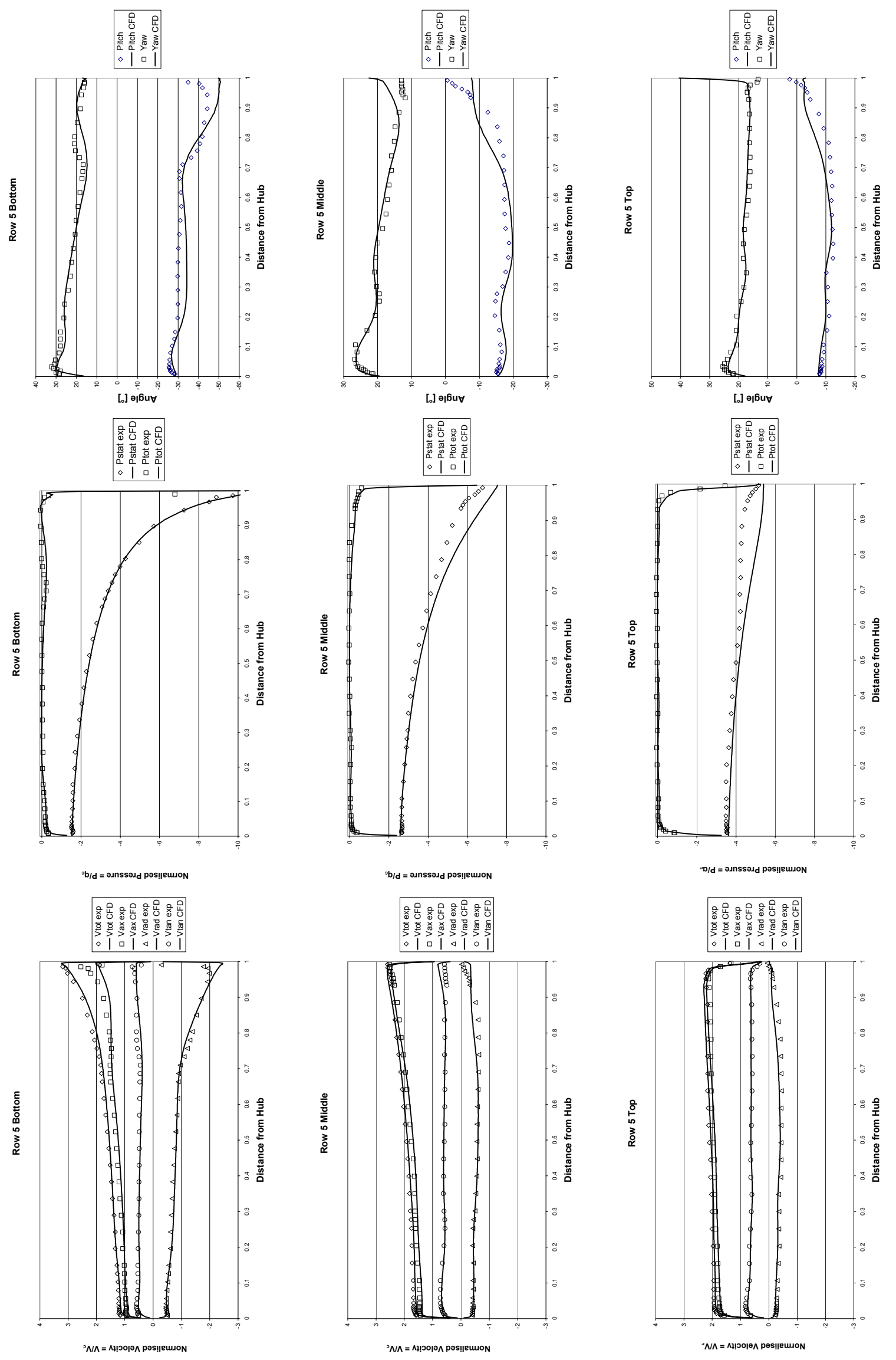

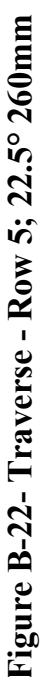



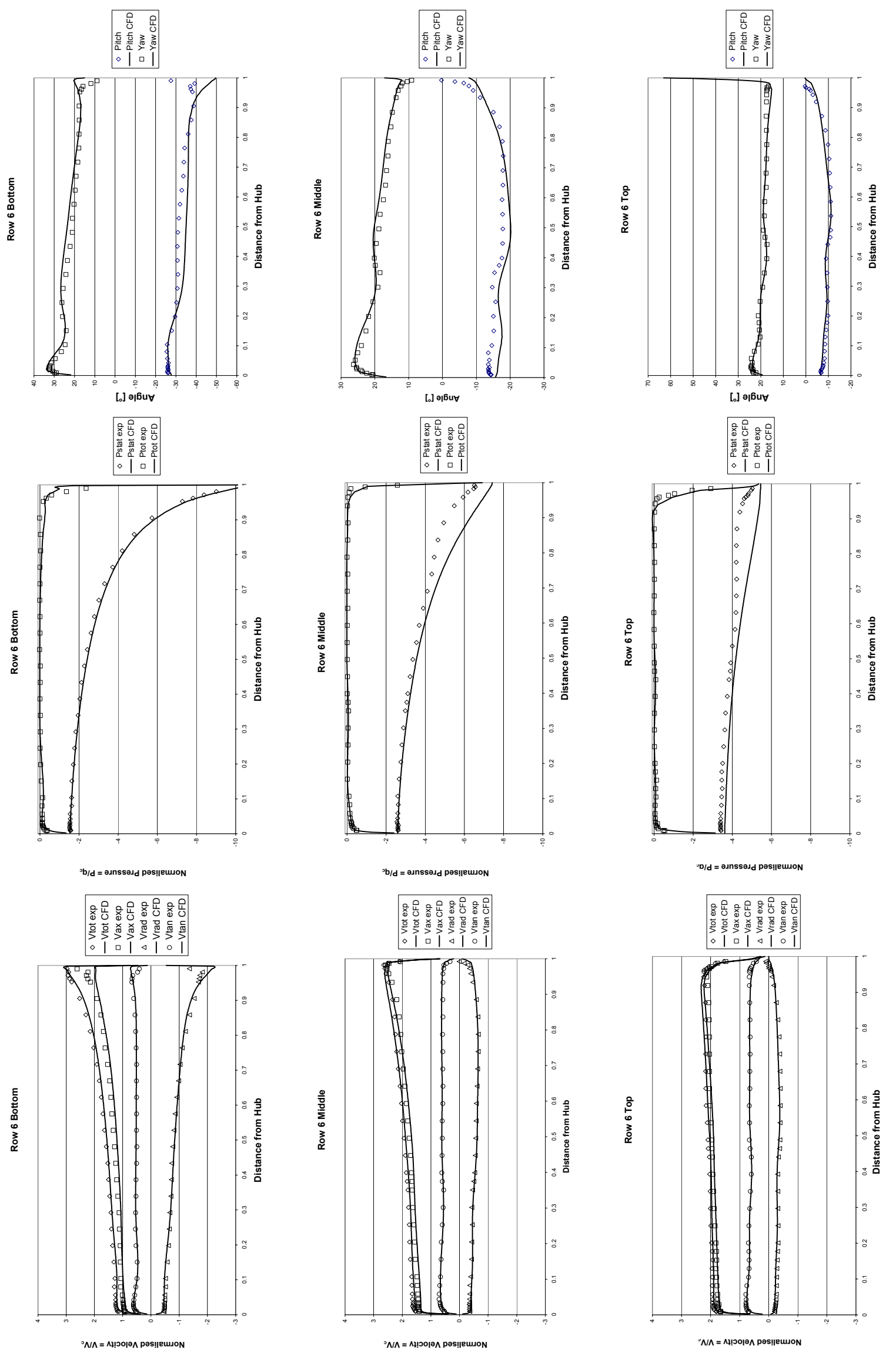

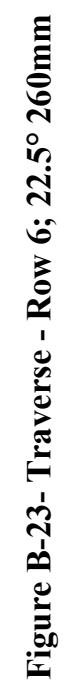



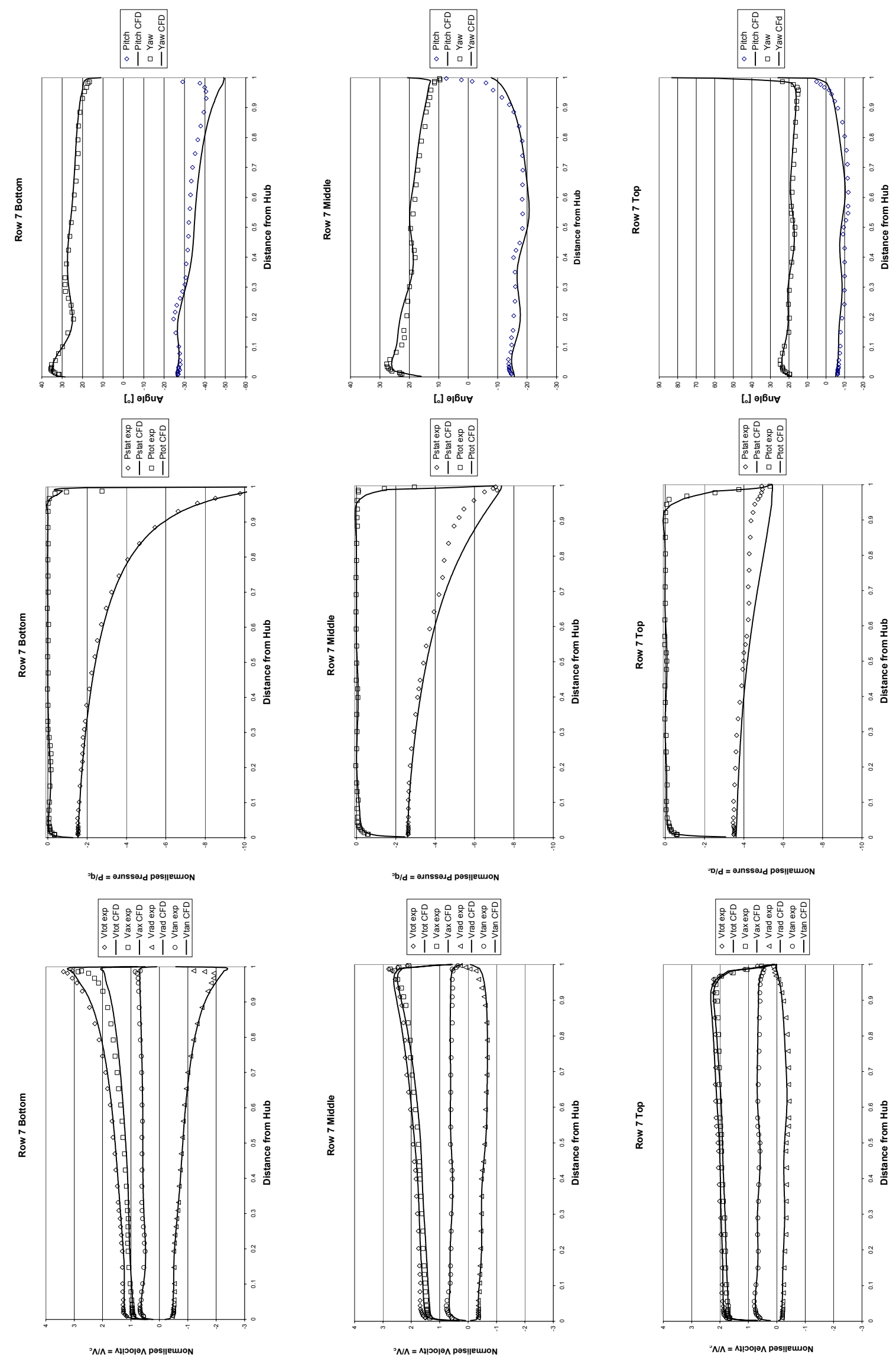

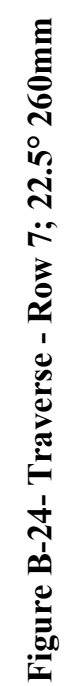



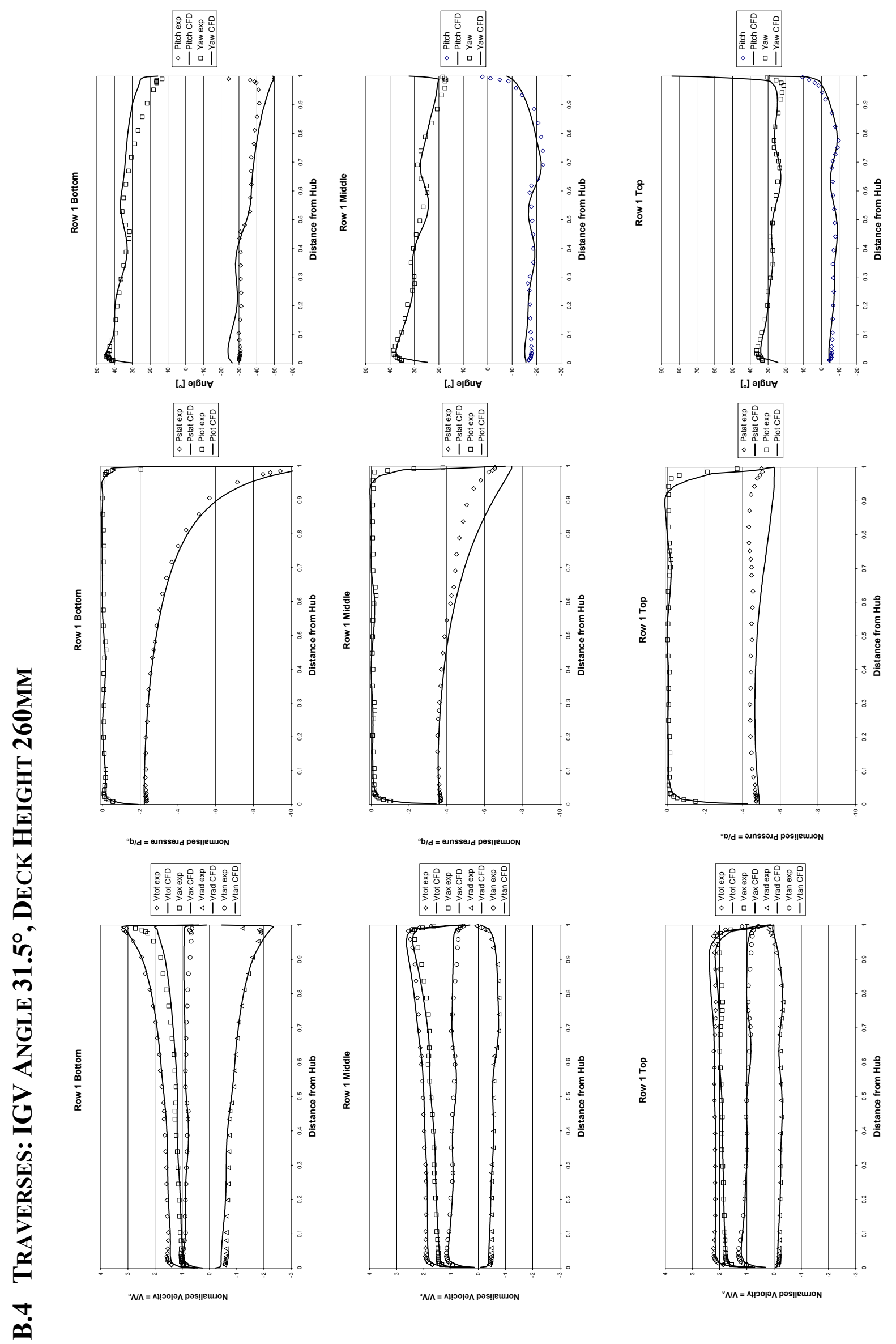

于
ஸ่
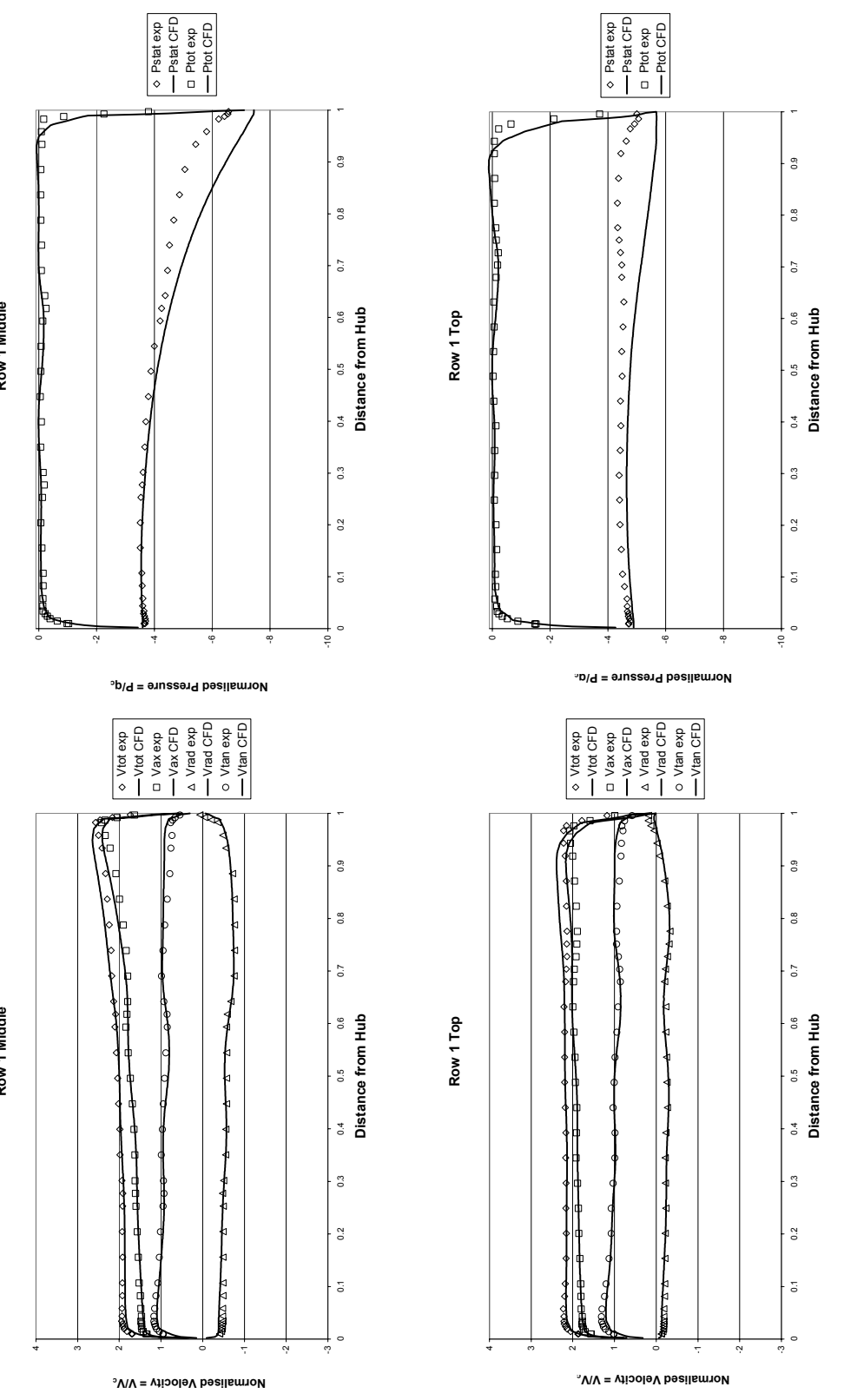

है 

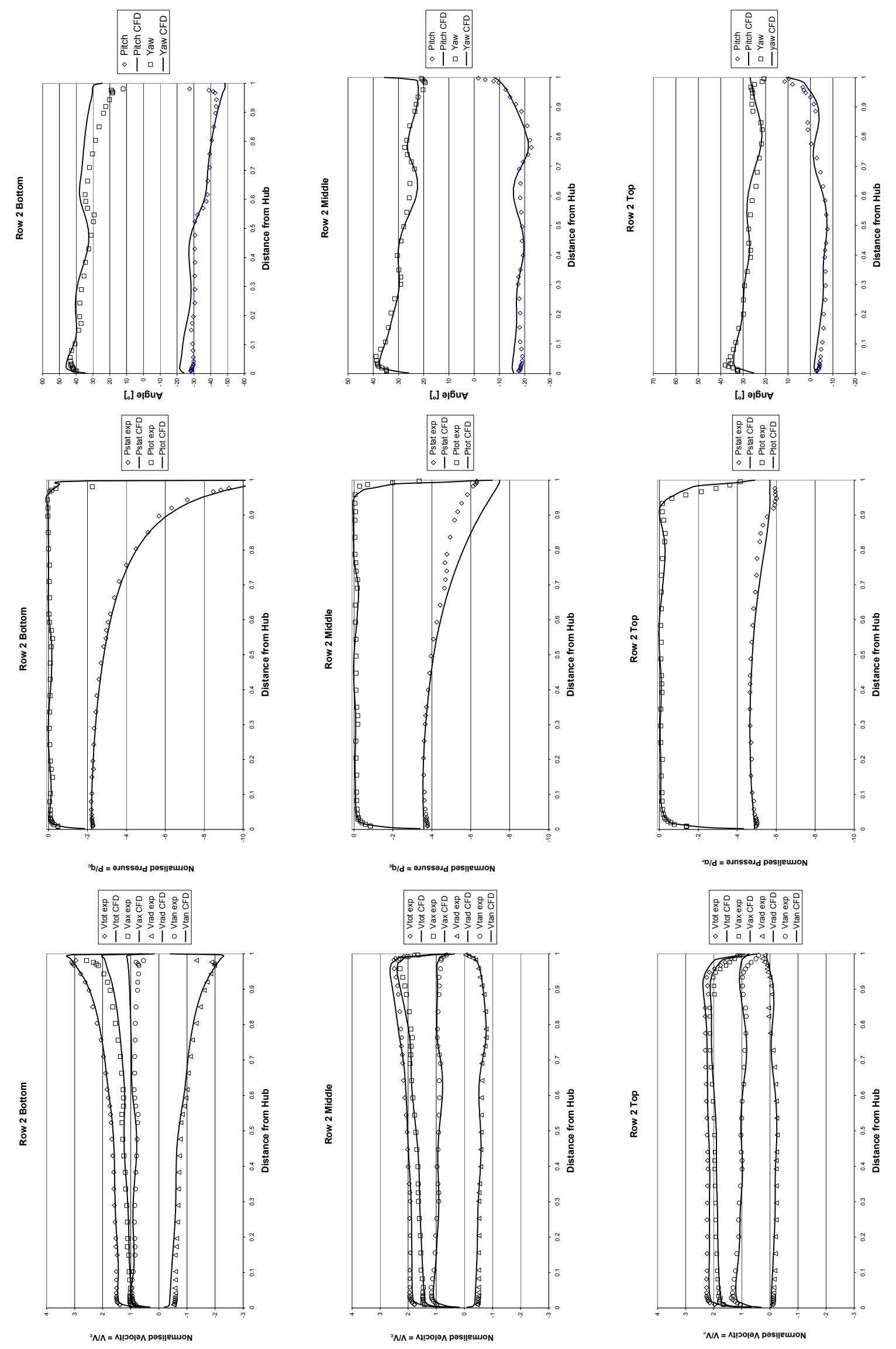

हैं 

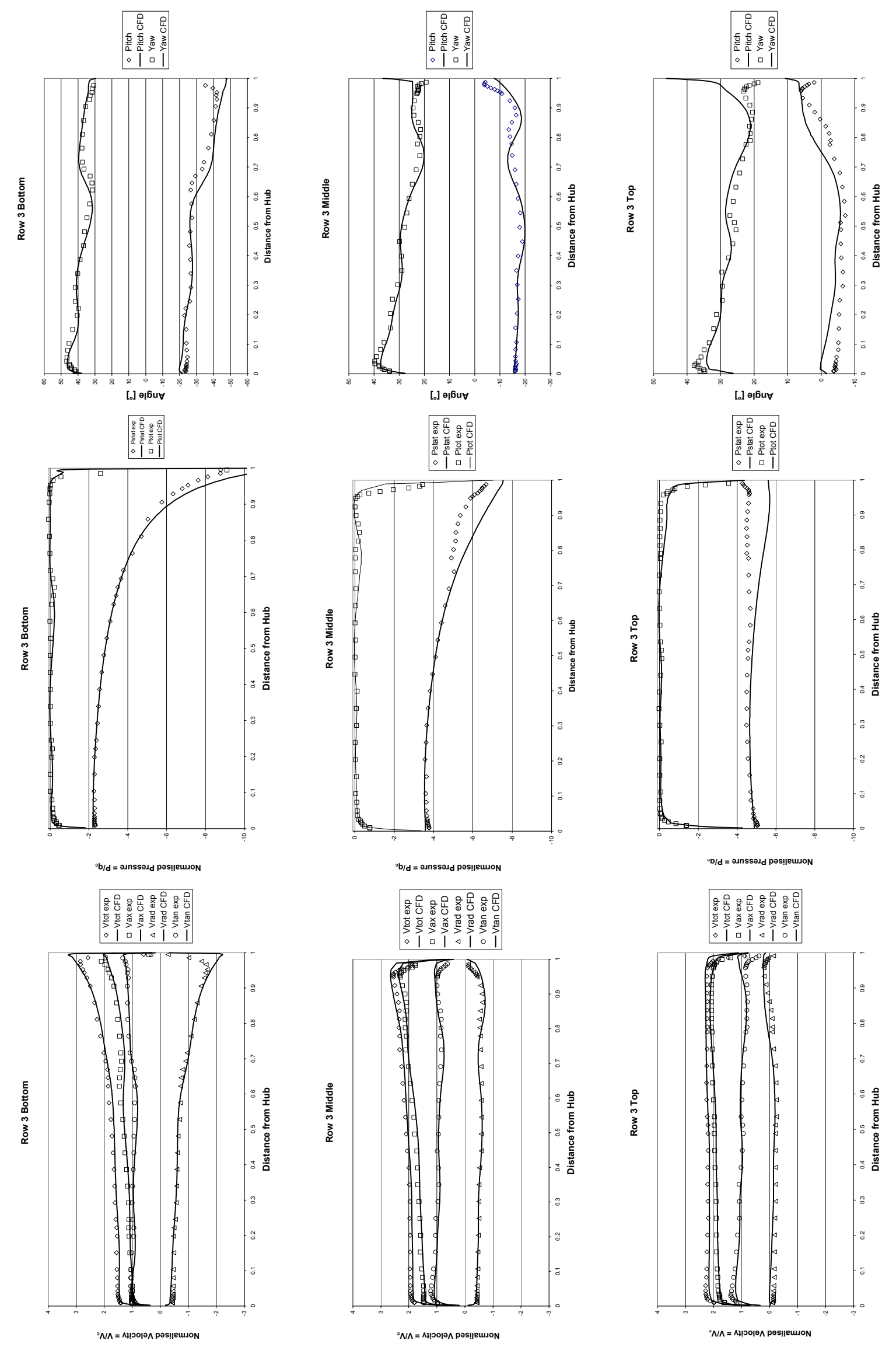

है 


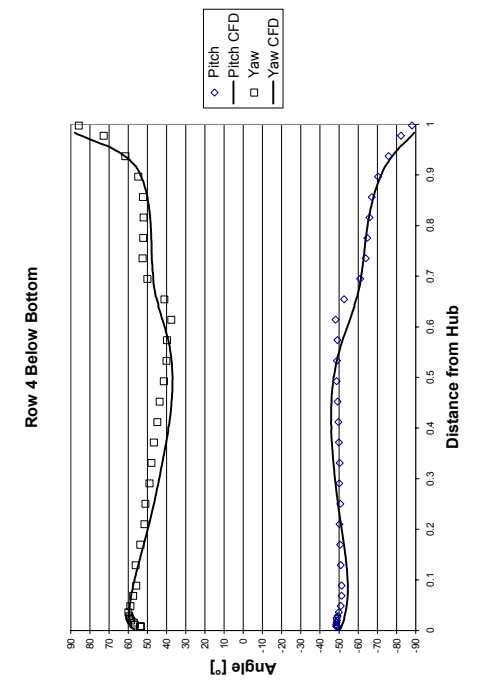

in

ம்
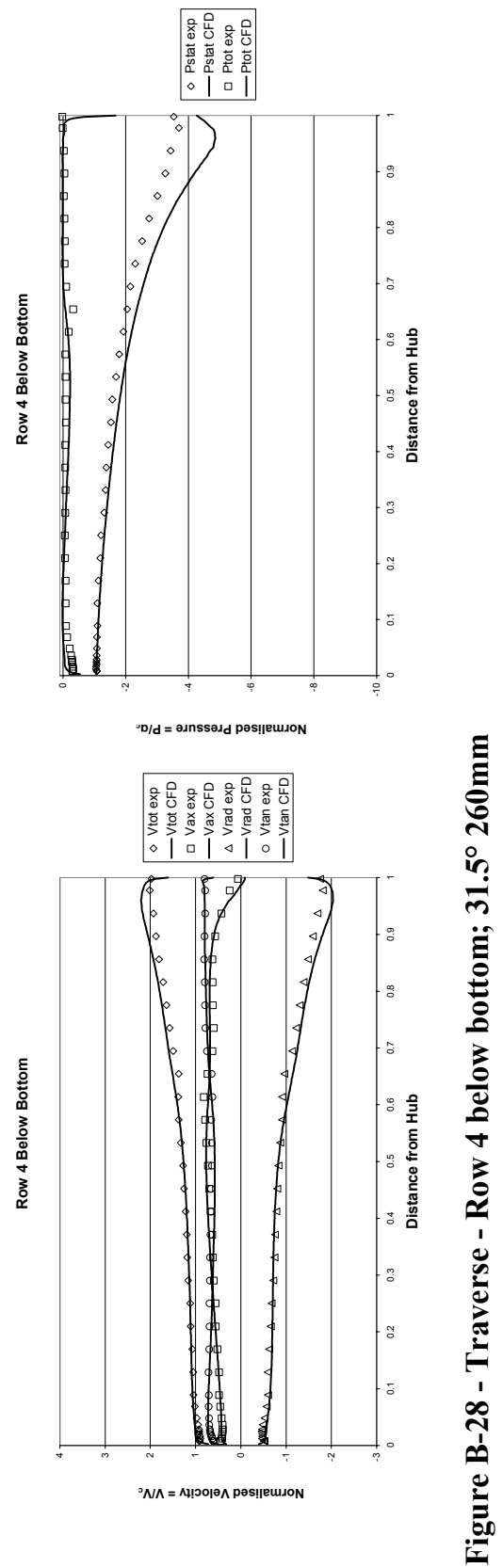

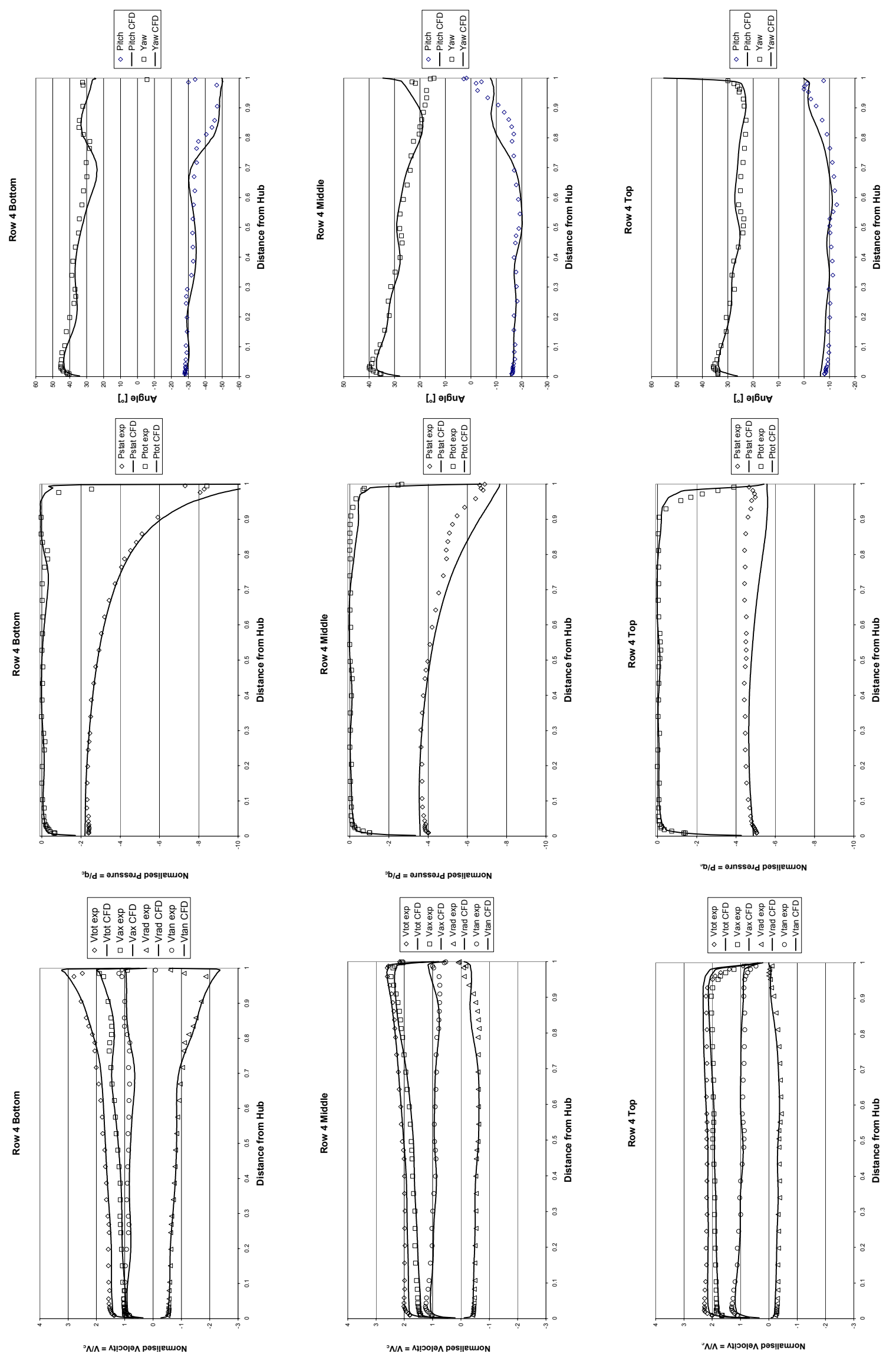

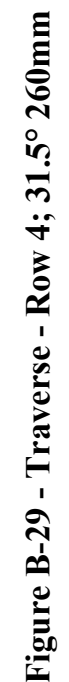



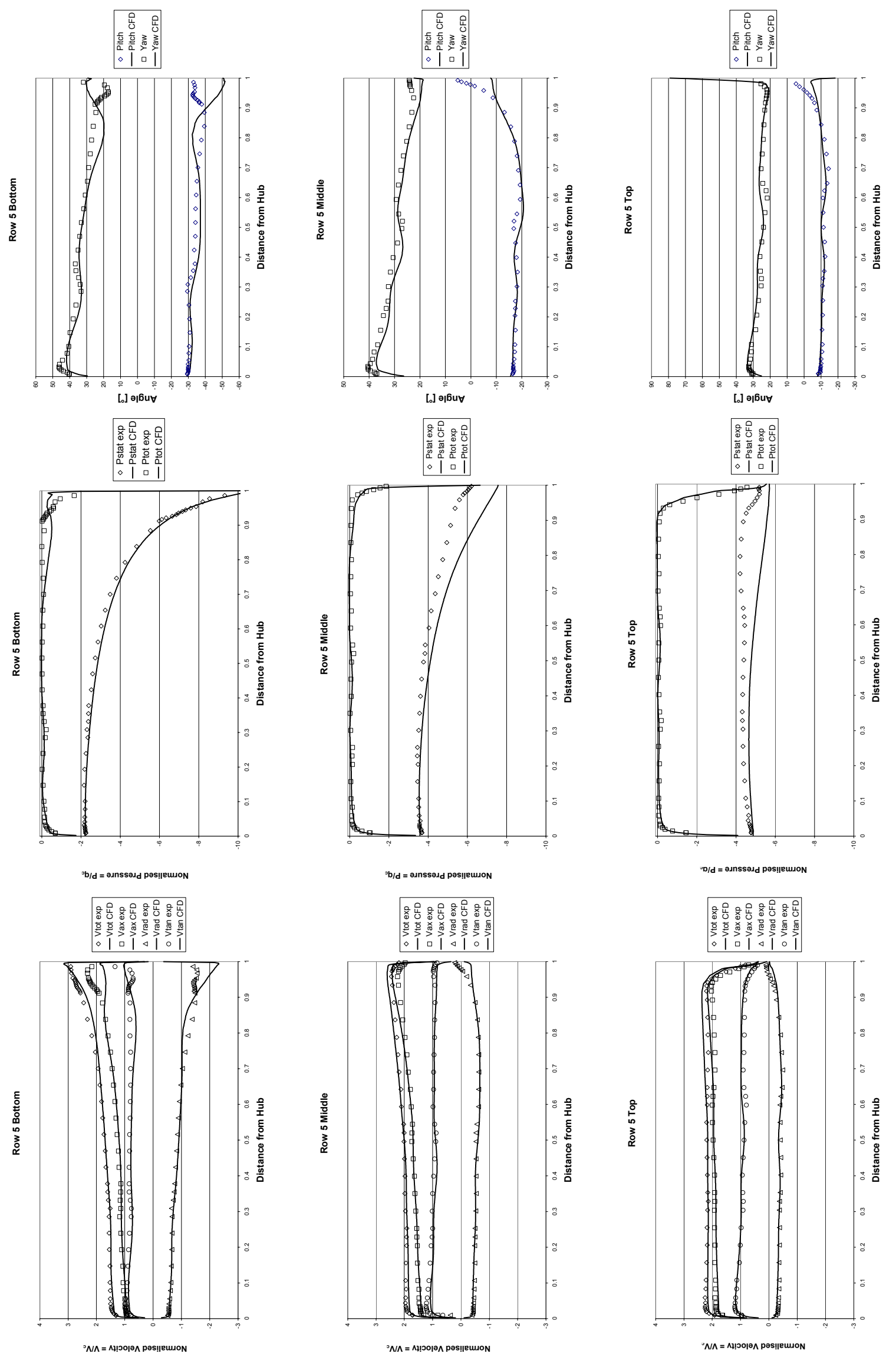

恶 

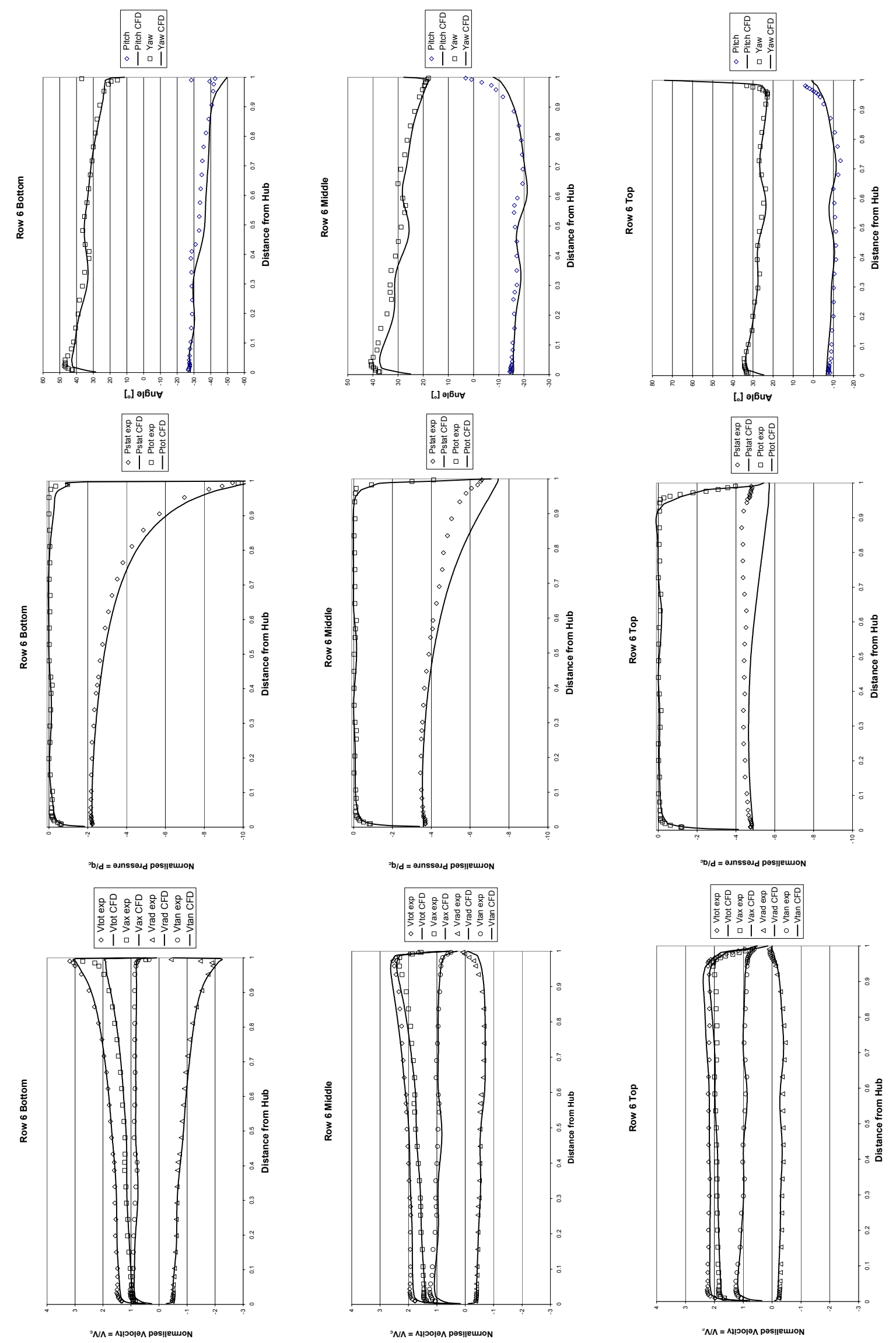

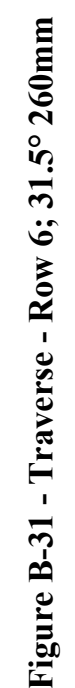



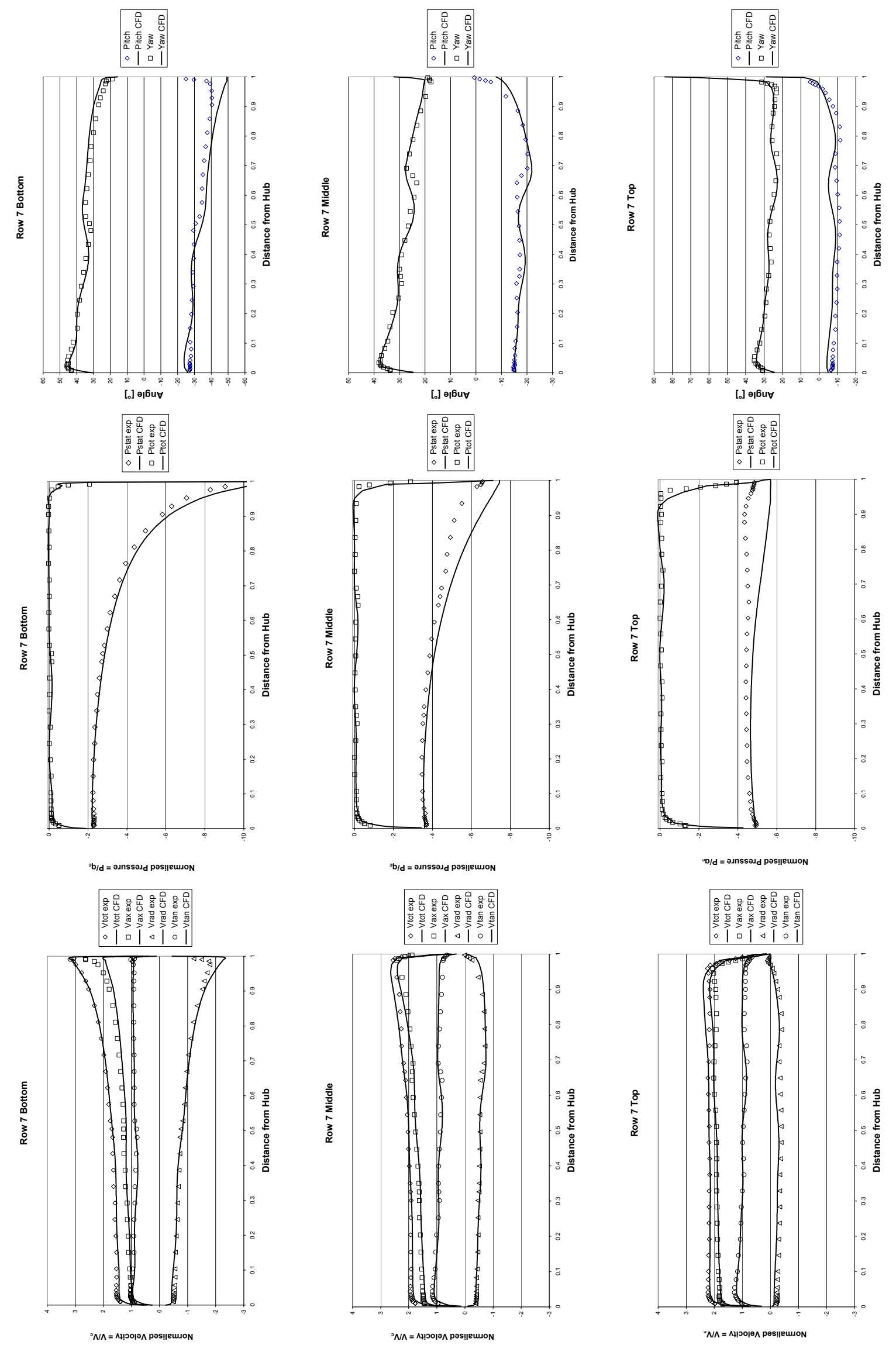

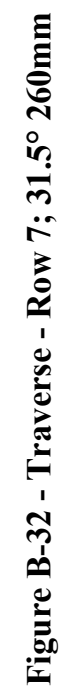




\section{B.5 OUter Wall: IGV ANGLe 22.5 $5^{\circ}$, DeCK Height 320Mm}

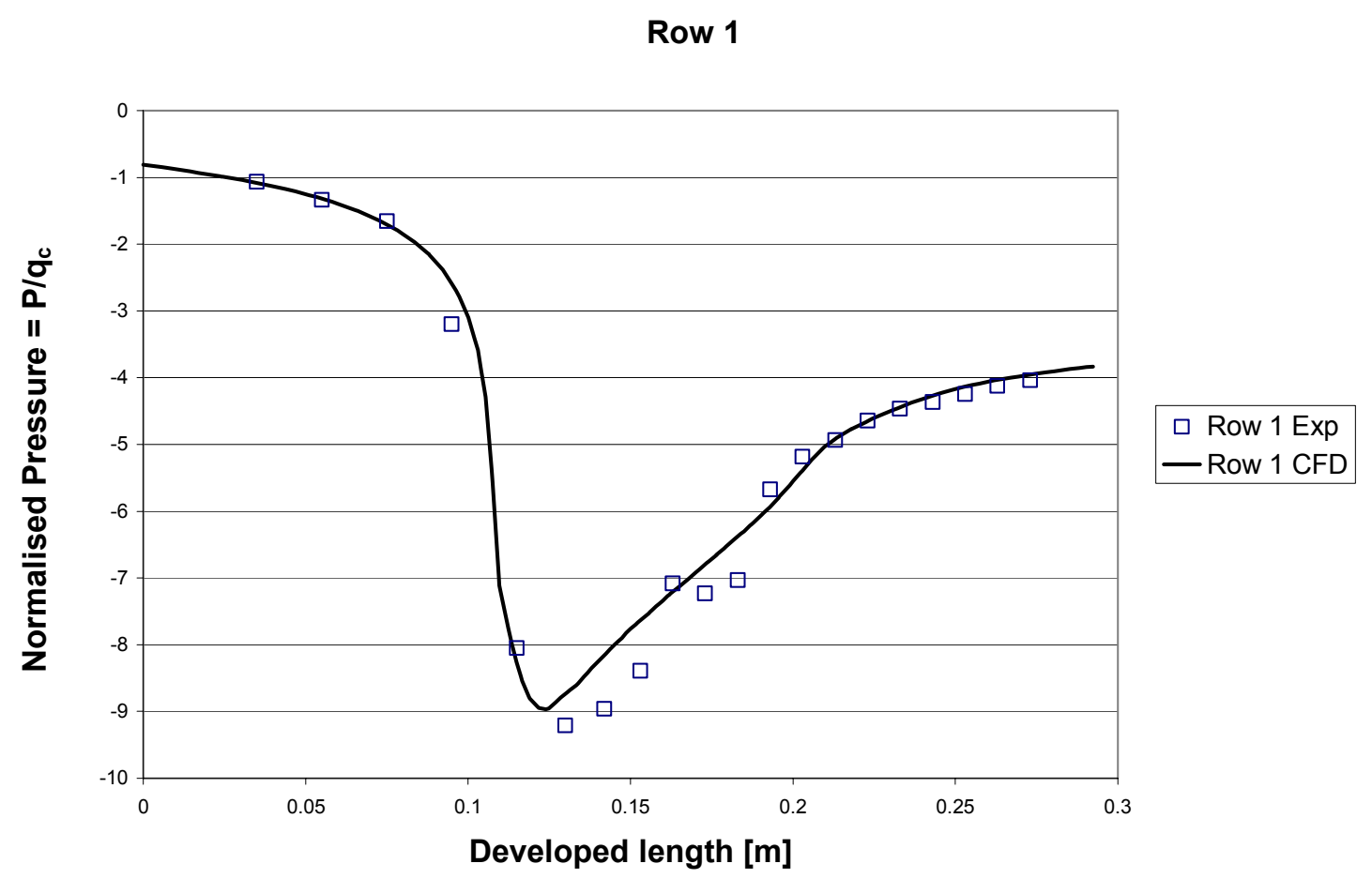

Figure B-33 - Outer wall - Row 1; 22.5 320mm

Row 2

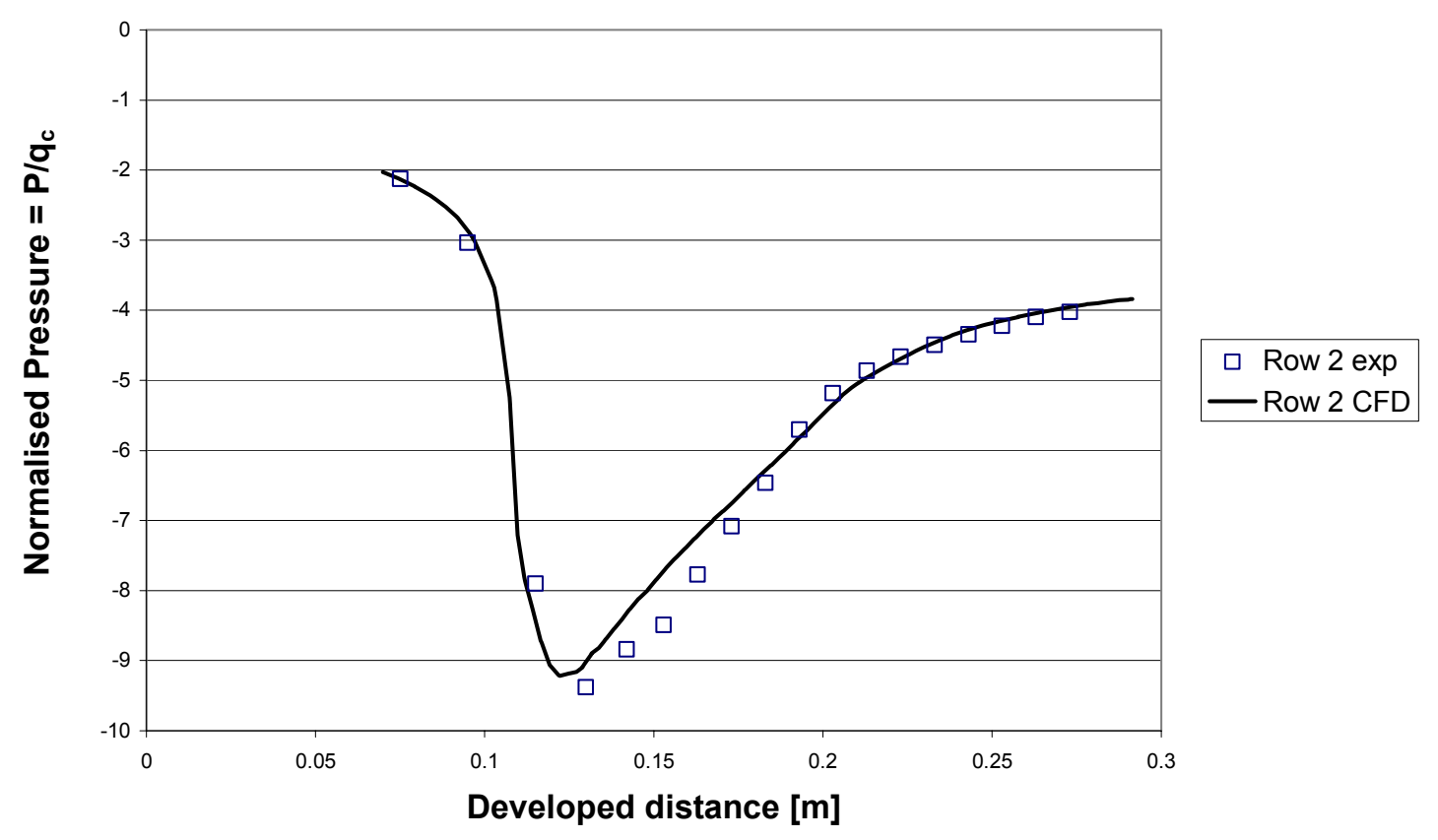

Figure B-34 - Outer wall - Row 2; $22.5^{\circ} 320 \mathrm{~mm}$ 
Row 3

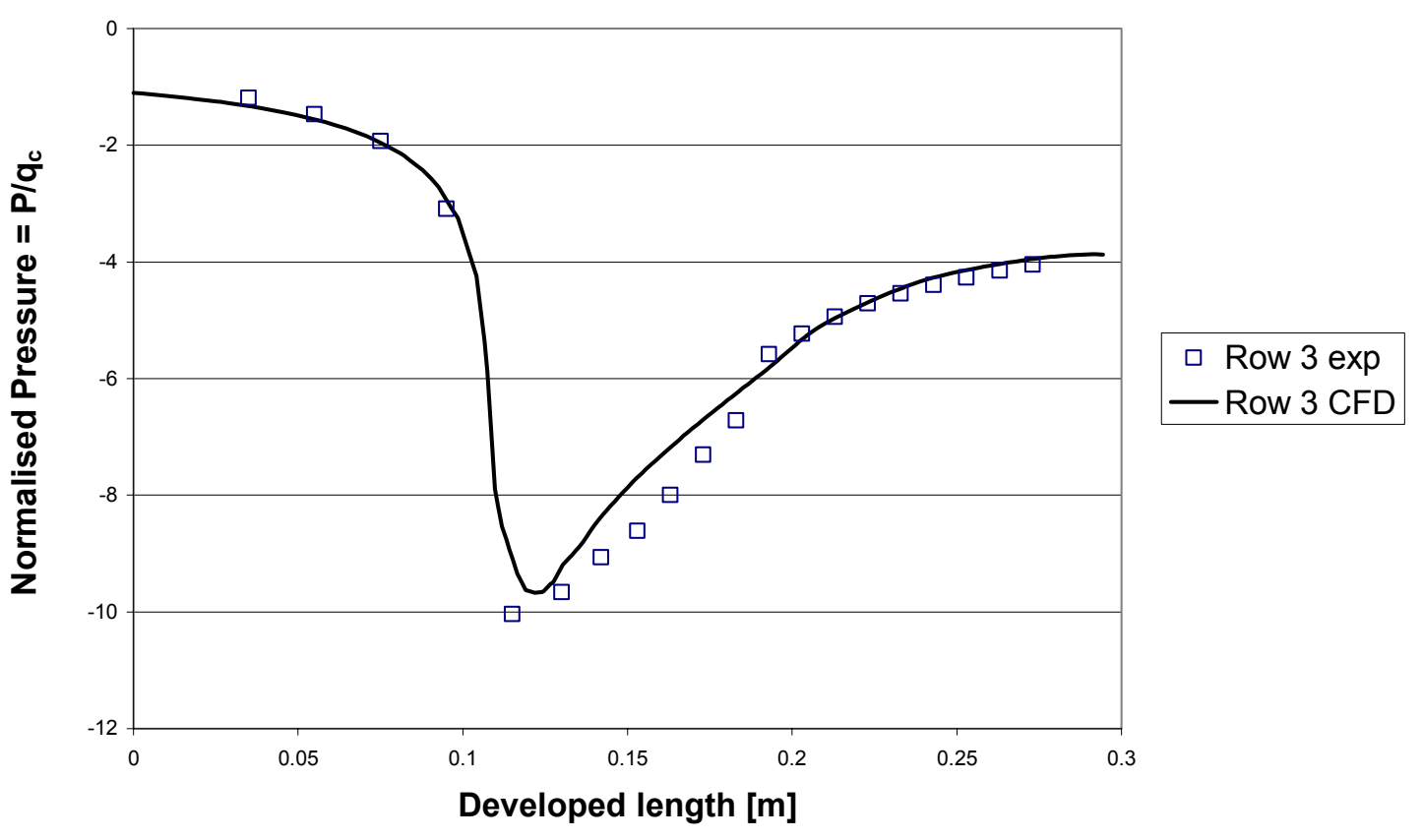

Figure B-35 - Outer wall - Row 3; $22.5^{\circ} 320 \mathrm{~mm}$

Row 4

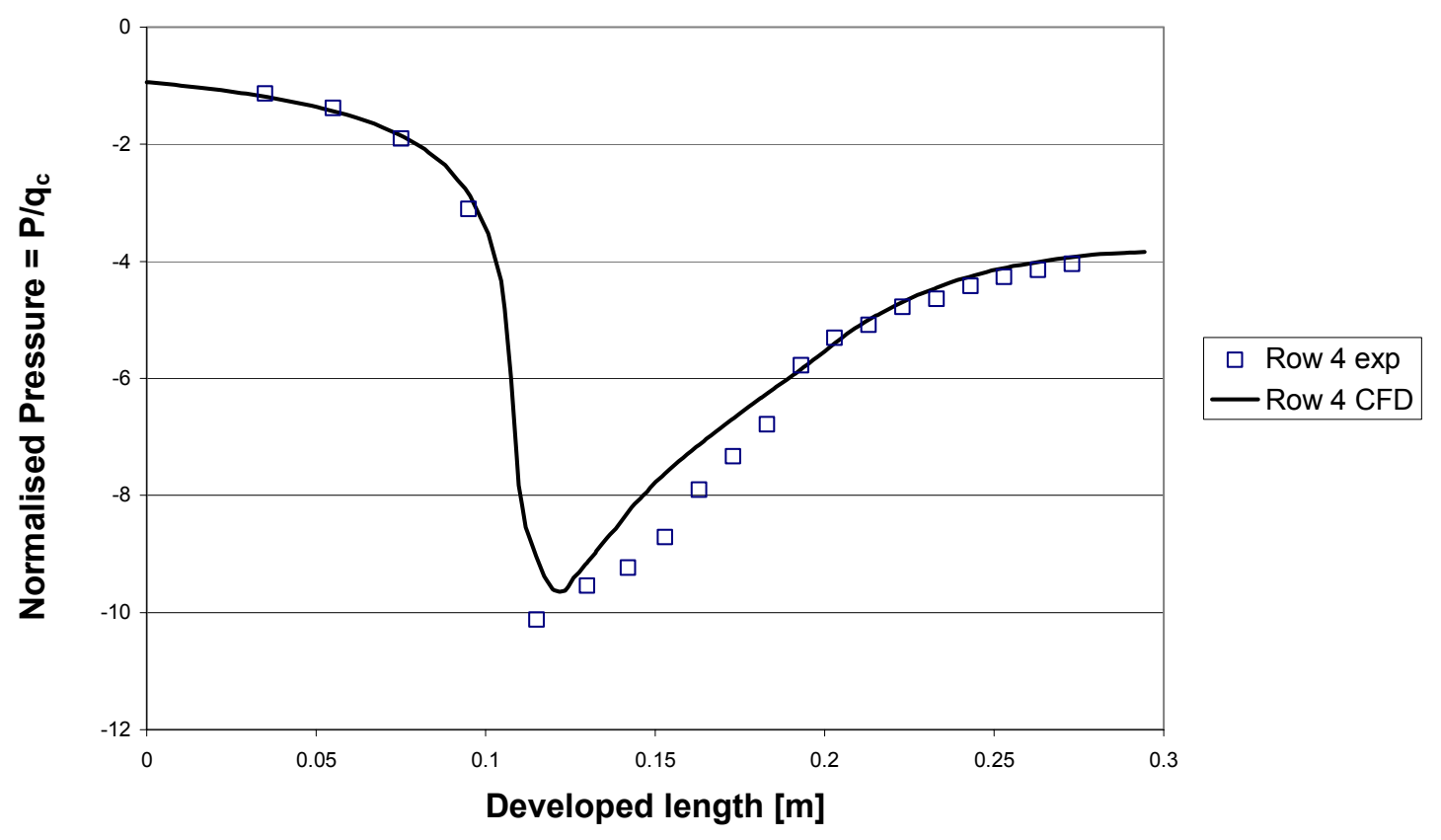

Figure B-36 - Outer wall - Row 4; $22.5^{\circ} 320 \mathrm{~mm}$ 
Row 5

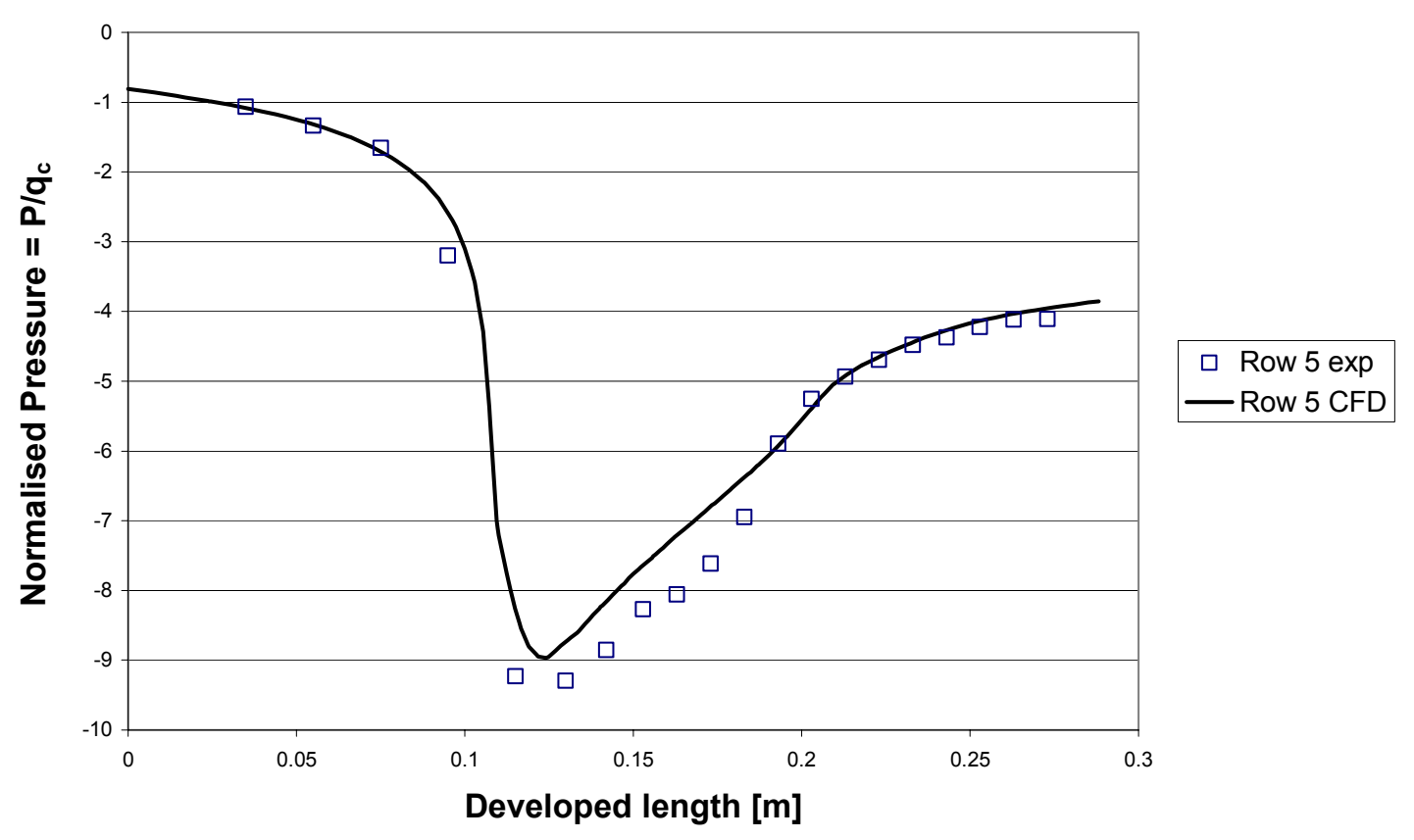

Figure B-37 - Outer wall - Row 5; $22.5^{\circ} 320 \mathrm{~mm}$

\section{B.6 Outer Wall: IGV ANGle 31.5 ${ }^{\circ}$, DeCK Height 320Mm}

\section{Row 1}

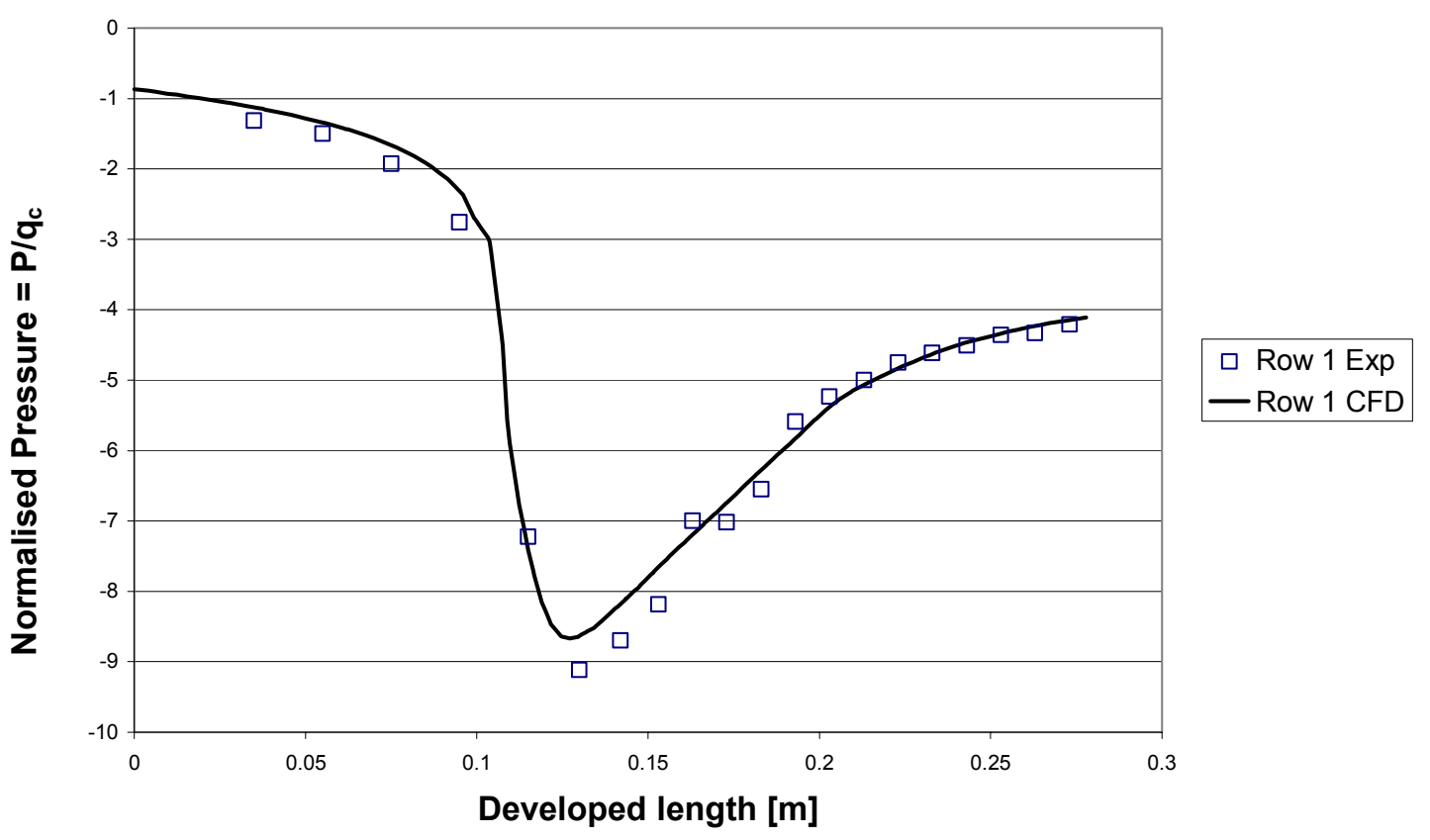

Figure B-38 - Outer wall - Row 1; 31.5 320mm 
Row 2

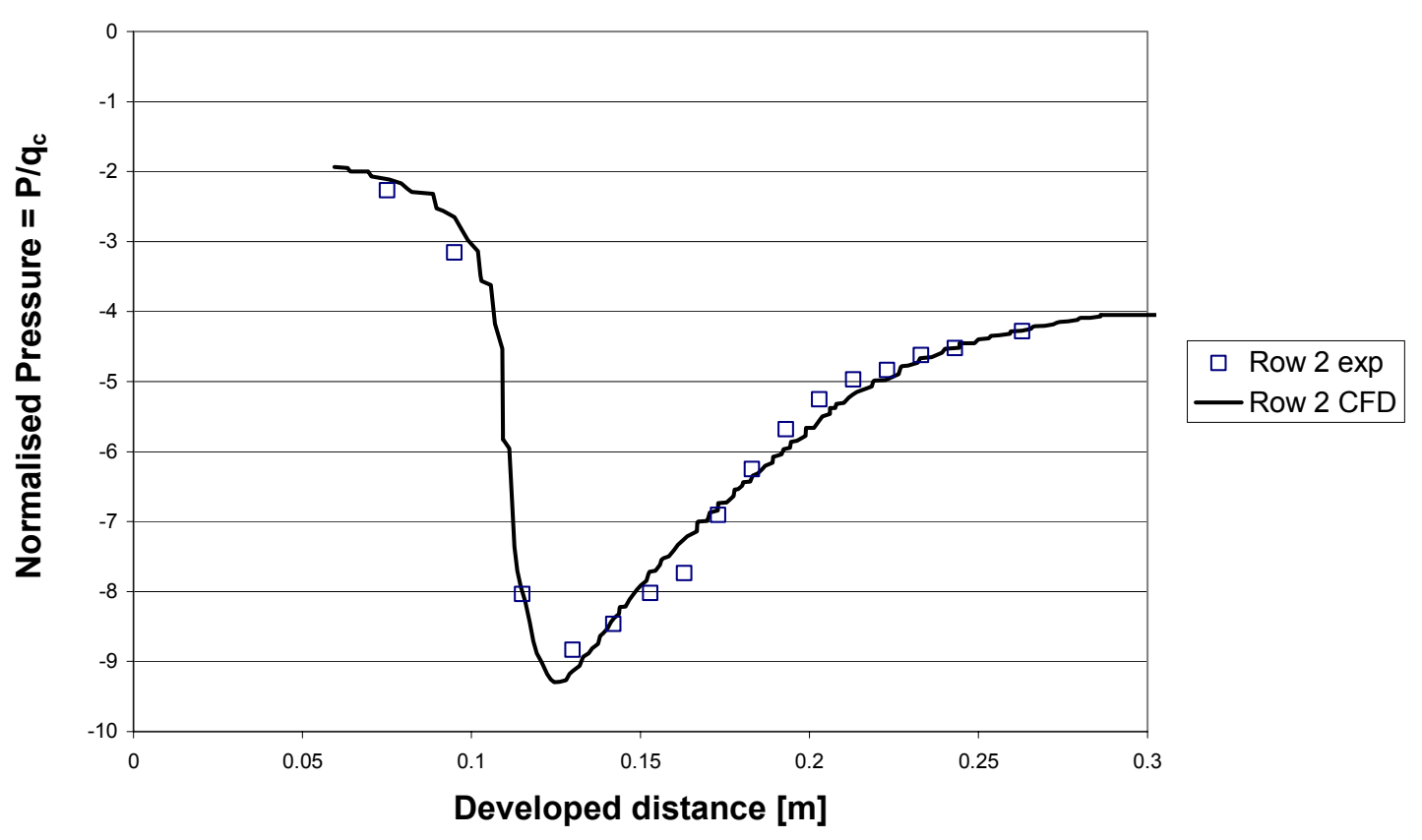

Figure B-39 - Outer wall - Row 2; 31.5 320mm

Row 3

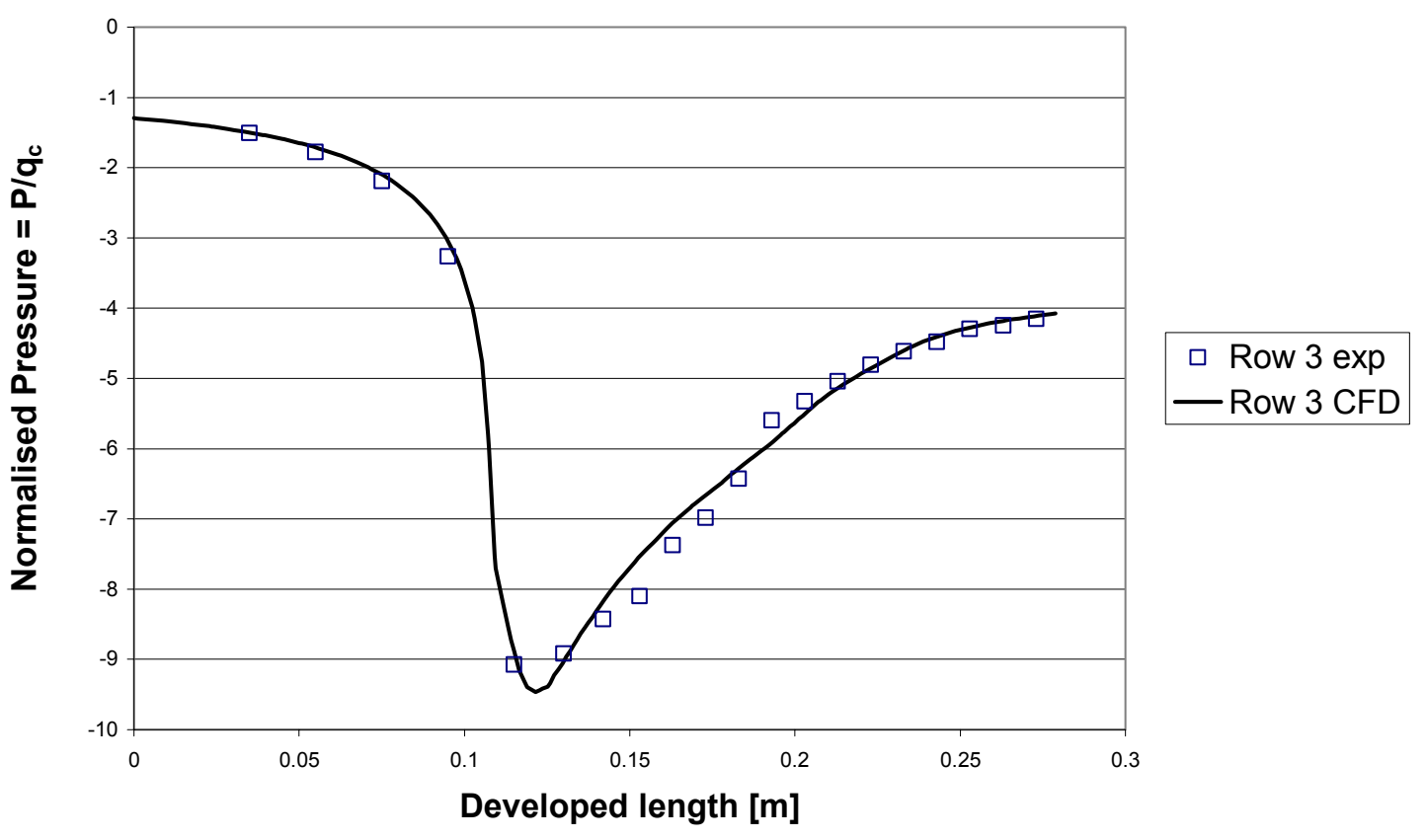

Figure B-40 - Outer wall - Row 3; 31.5 $320 \mathrm{~mm}$ 
Row 4

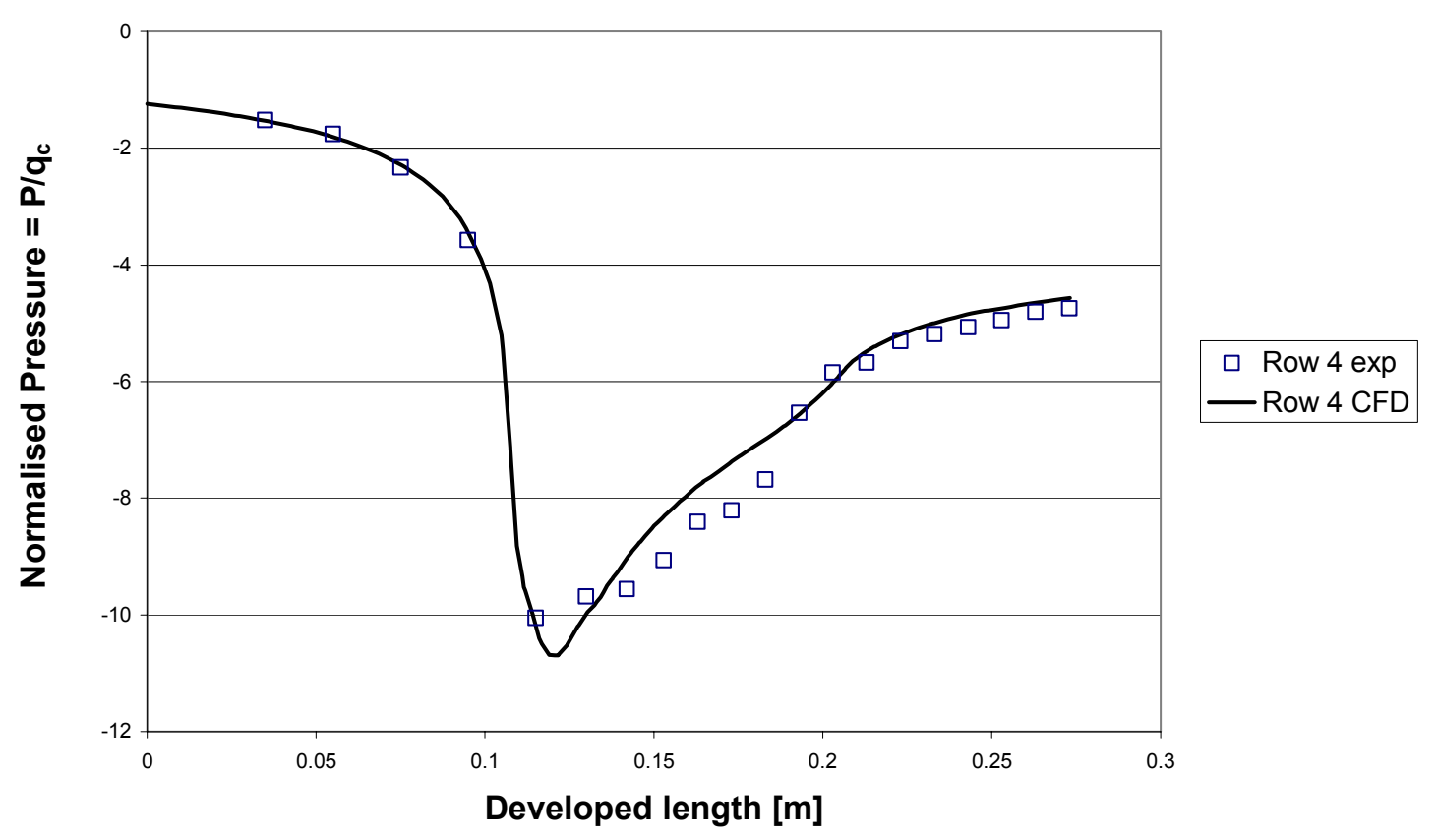

Figure B-41 - Outer wall - Row 4; 31.5 $320 \mathrm{~mm}$

Row 5

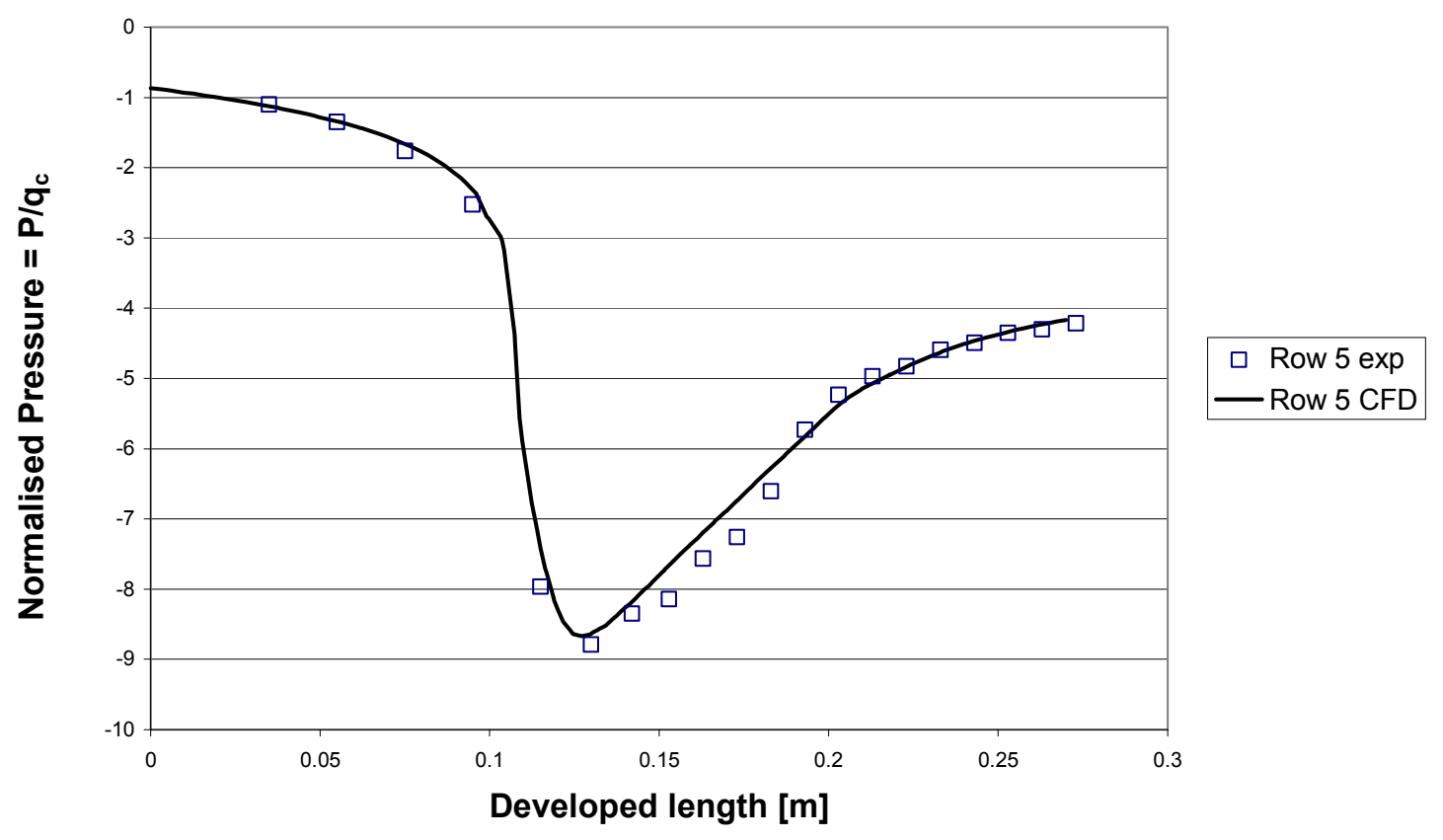

Figure B-42 - Outer wall - Row 5; 31.5 $320 \mathrm{~mm}$ 


\section{B.7 Outer Wall: IGV ANGle 22.5 $5^{\circ}$, DeCK Height 260MM}

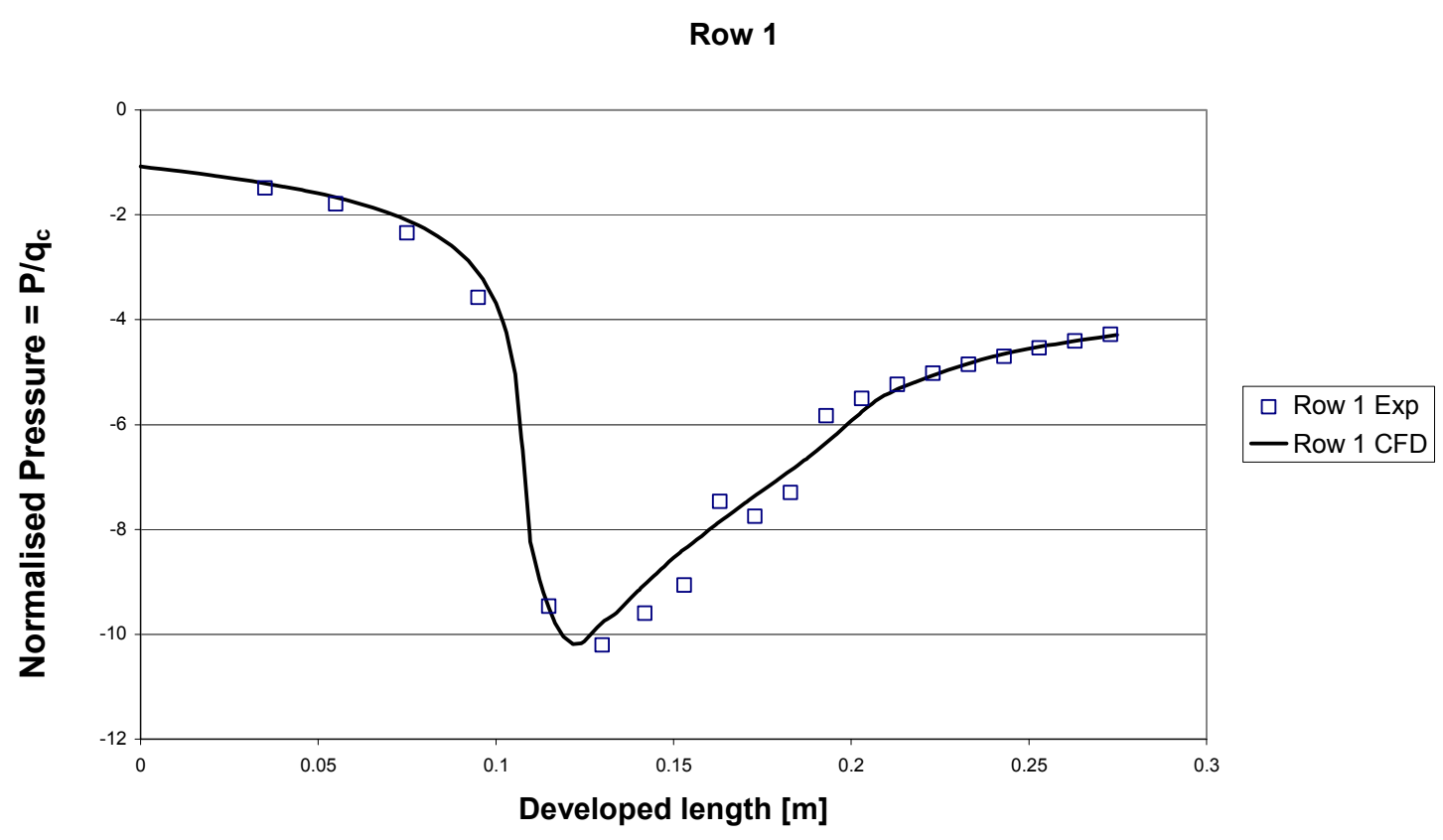

Figure B-43 - Outer wall - Row 1; $22.5^{\circ} 260 \mathrm{~mm}$

Row 2

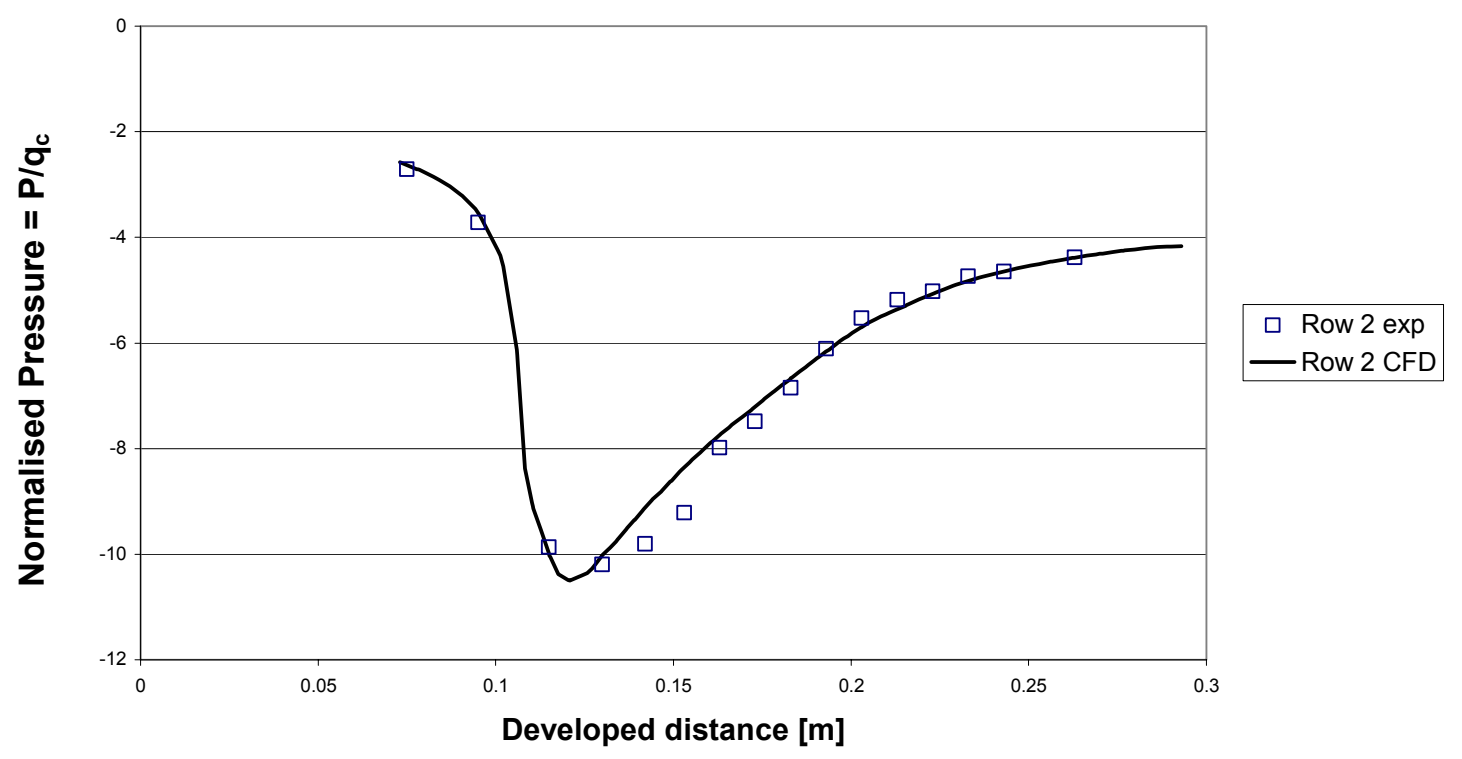

Figure B-44 - Outer wall - Row 2; $22.5^{\circ} 260 \mathrm{~mm}$ 
Row 3

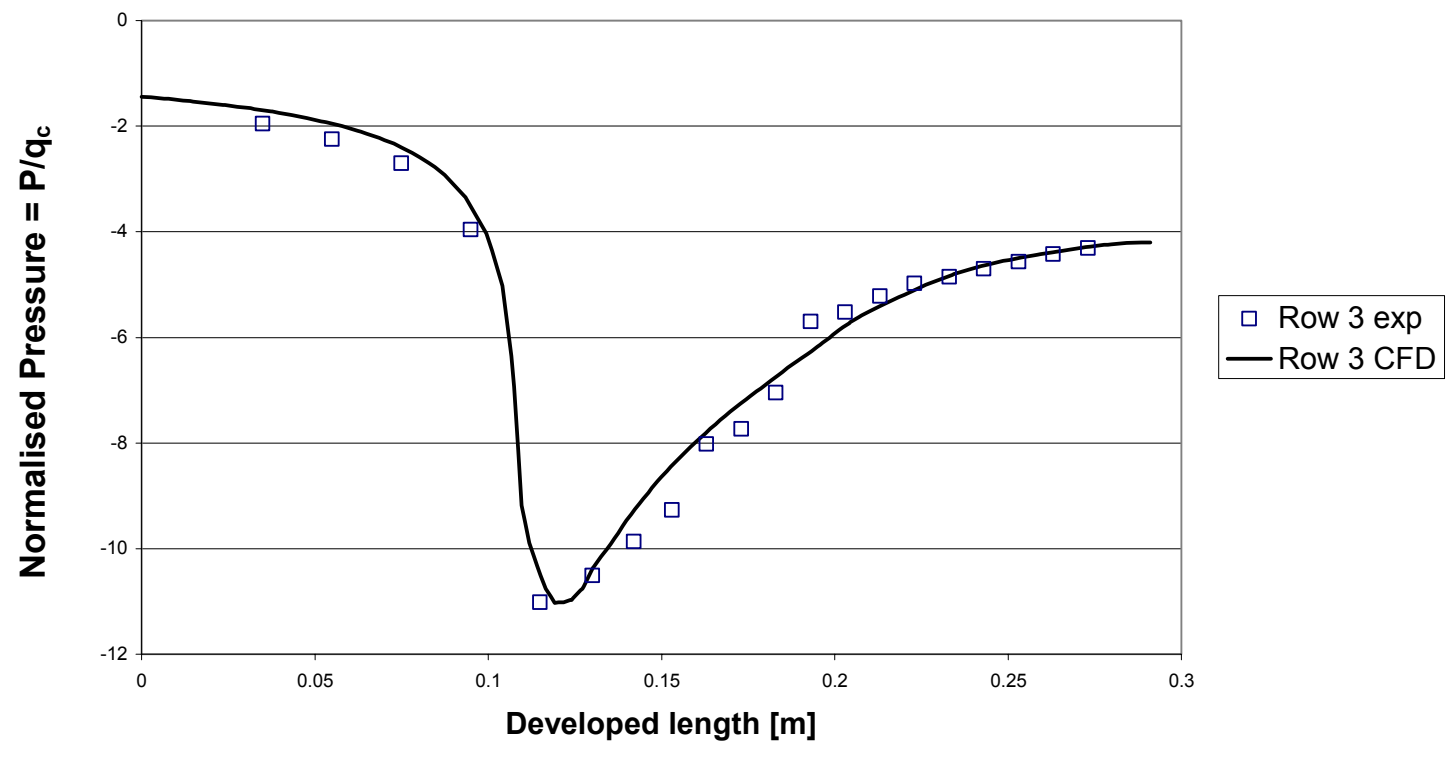

Figure B-45 - Outer wall - Row 3; $22.5^{\circ} 260 \mathrm{~mm}$

Row 4

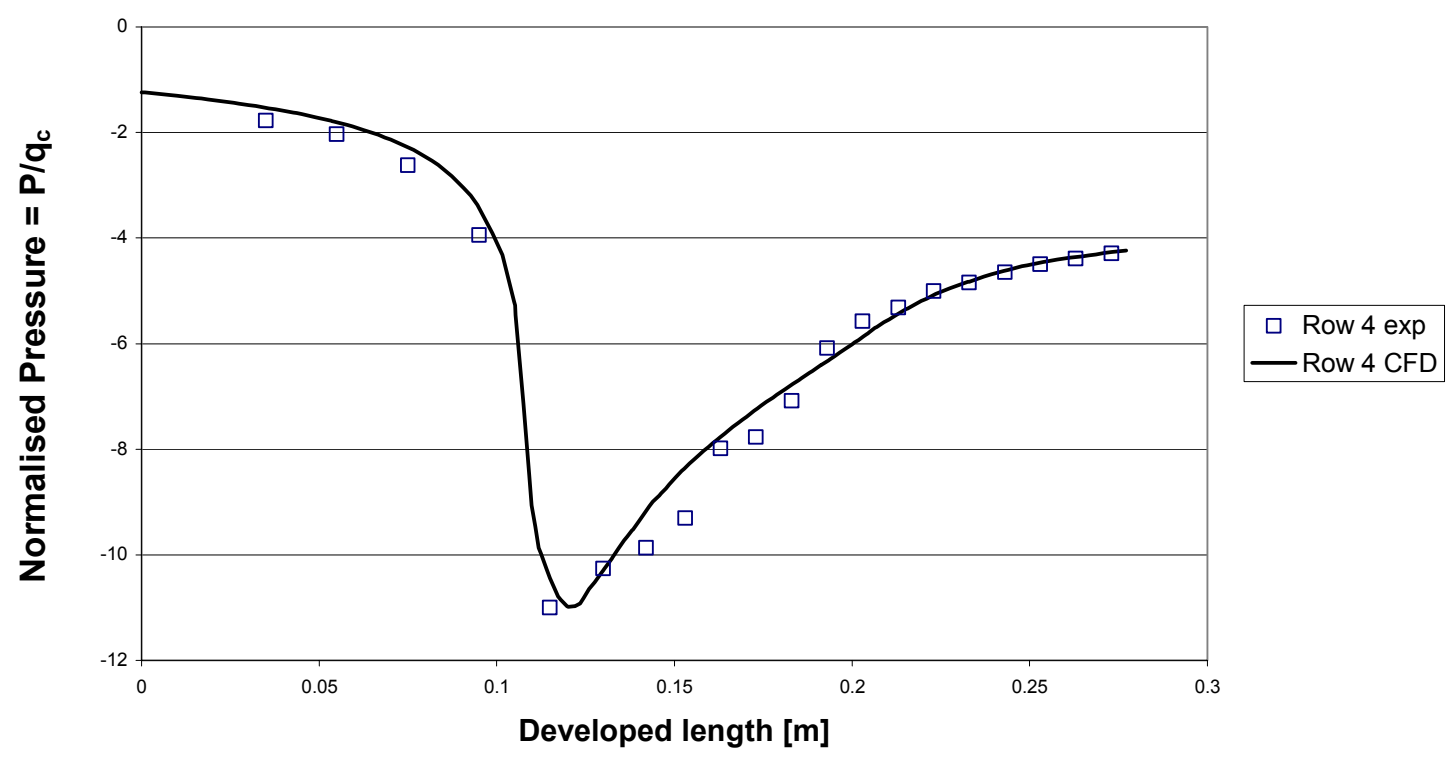

Figure B-46 - Outer wall - Row 4; $22.5^{\circ} 260 \mathrm{~mm}$ 
Row 5

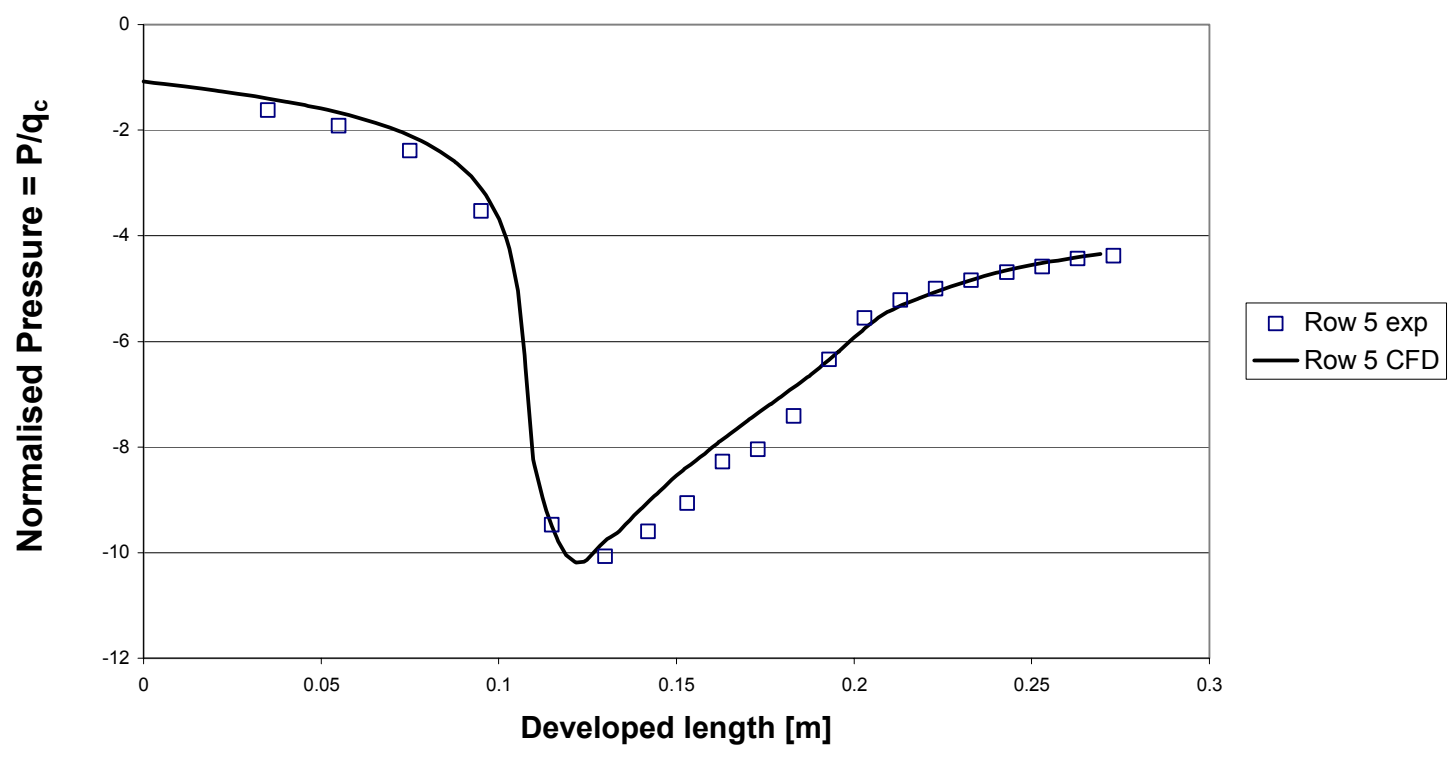

Figure B-47 - Outer wall - Row 5; 22.5 260mm

\section{B.8 OUter WALl: IGV ANGLe 31.5 ${ }^{\circ}$, DeCK Height 260MM}

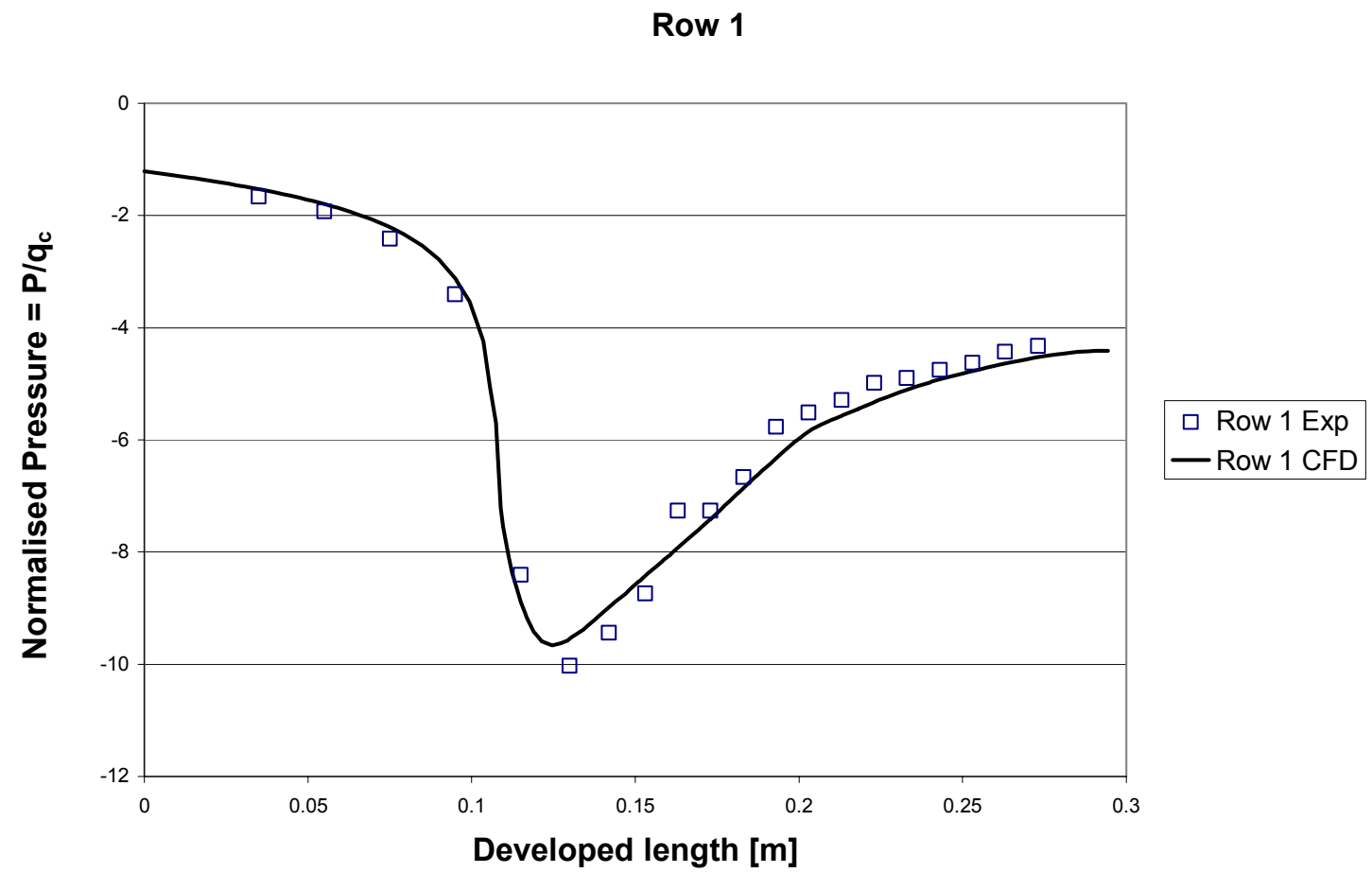

Figure B-48 - Outer wall - Row 1; 31.5 260mm 
Row 2

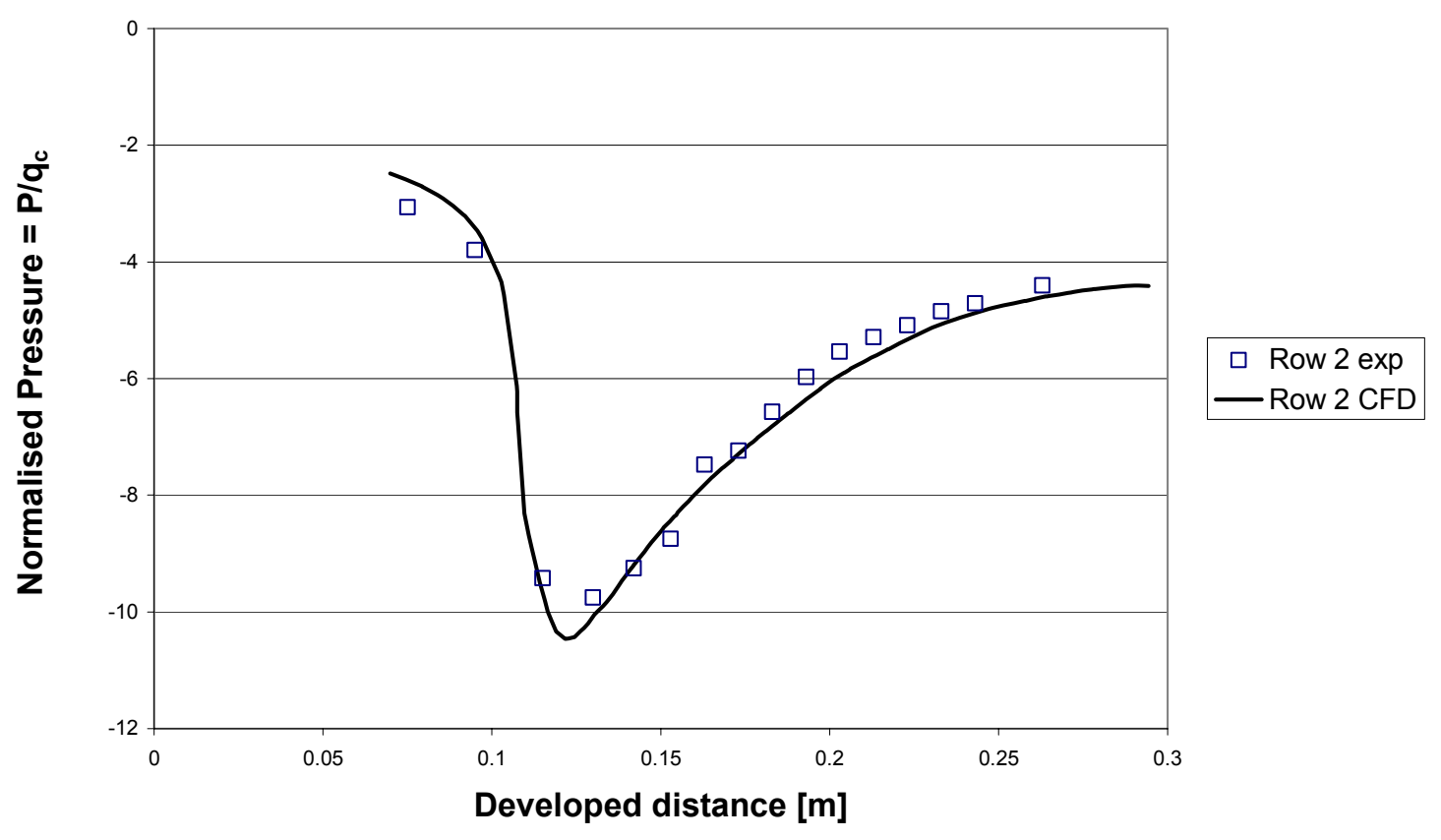

Figure B-49 - Outer wall - Row 2; 31.5 $260 \mathrm{~mm}$

Row 3

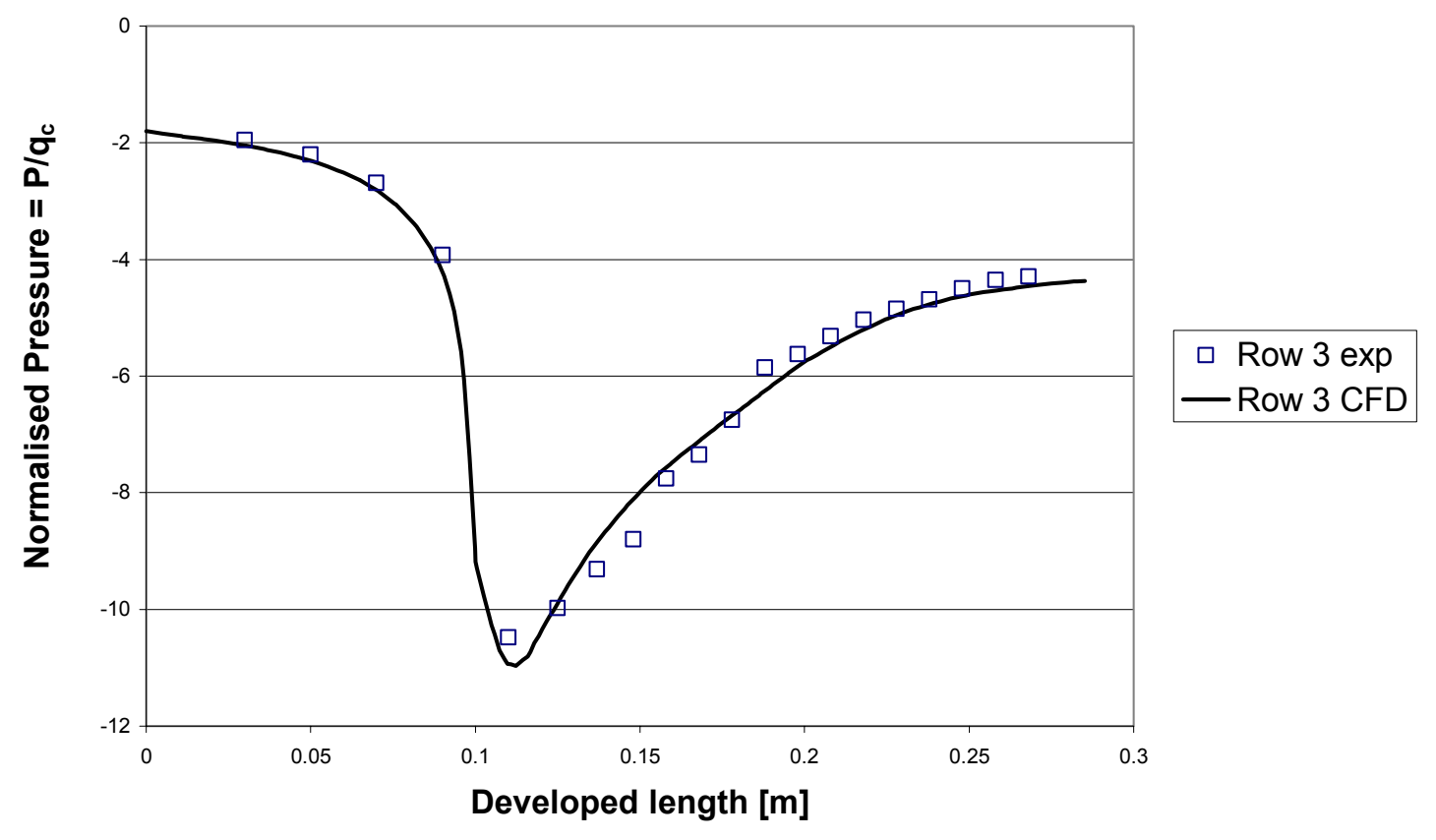

Figure B-50 - Outer wall - Row 3; 31.5 $260 \mathrm{~mm}$ 
Row 4

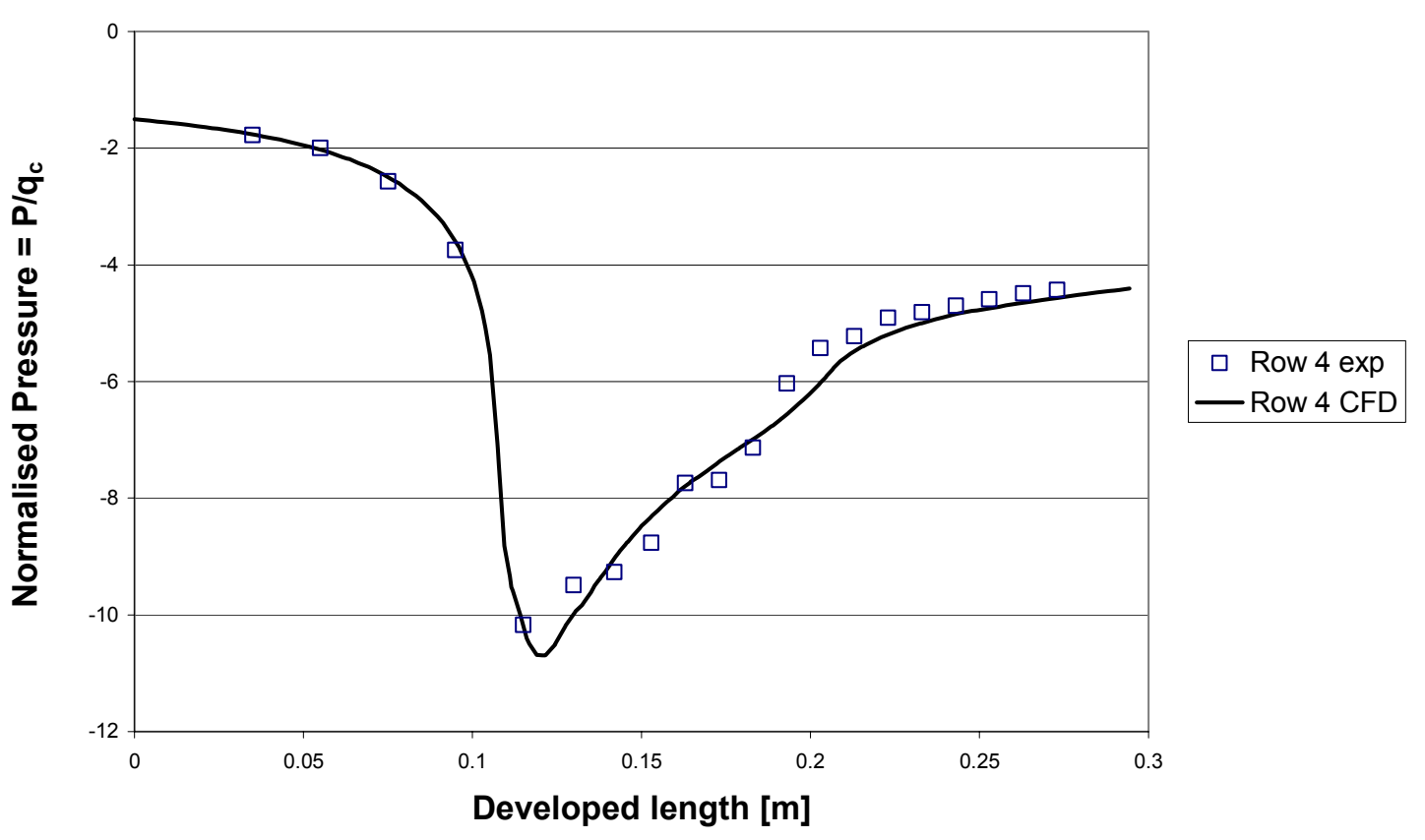

Figure B-51 - Outer wall - Row 4; 31.5 $260 \mathrm{~mm}$

Row 5

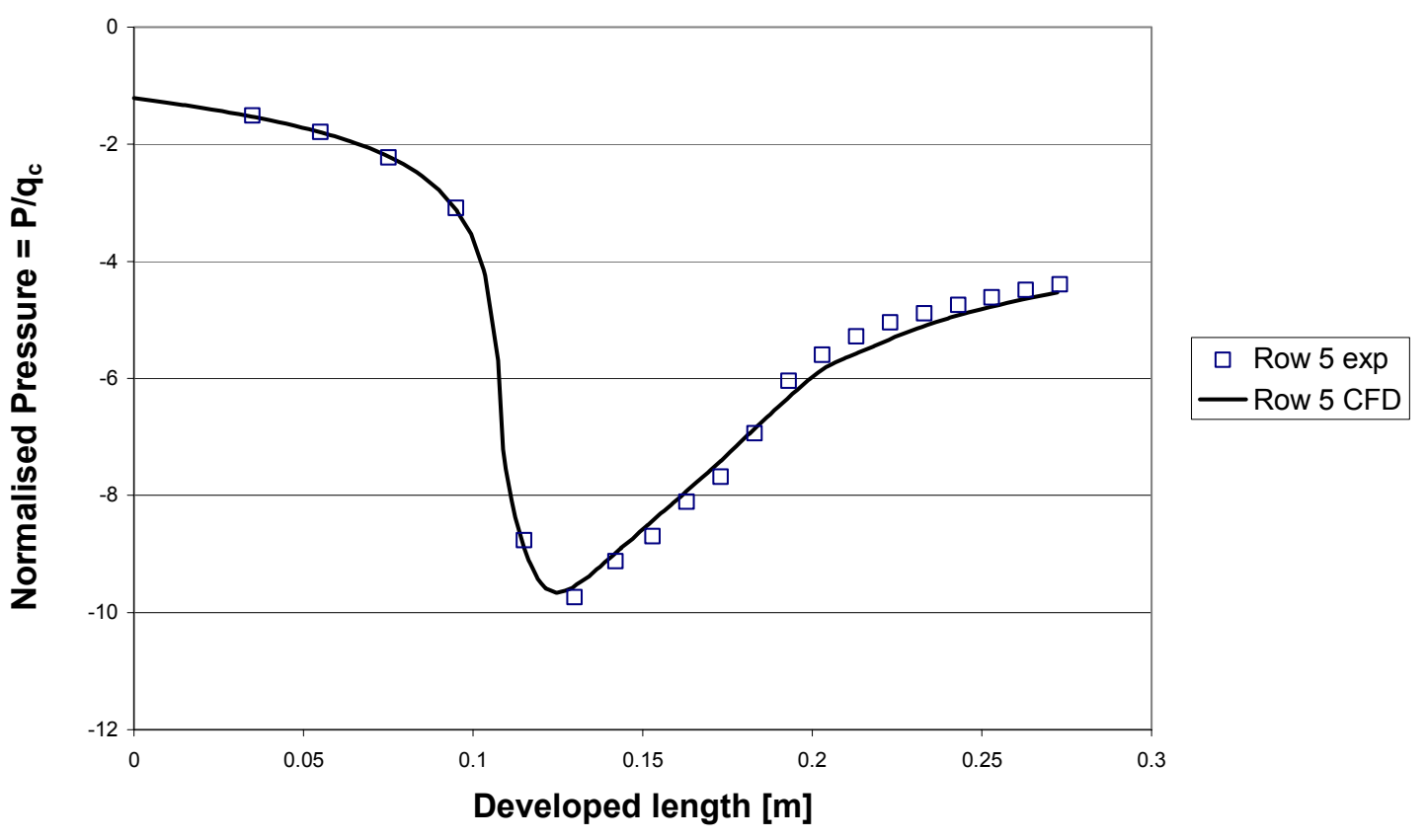

Figure B-52 - Outer wall - Row 5; 31.5 $260 \mathrm{~m}$ 